Argonne

\title{
Annual Report of Groundwater Monitoring at Everest, Kansas, in 2013
}

\section{Environmental Science Division}


About Argonne National Laboratory

Argonne is a U.S. Department of Energy laboratory managed by UChicago Argonne, LLC under contract DE-AC02-06CH11357. The Laboratory's main facility is outside Chicago, at 9700 South Cass Avenue, Argonne, Illinois 60439. For information about Argonne and its pioneering science and technology programs, see www.anl.gov.

\section{Availability of This Report}

This report is available, at no cost, at http://www.osti.gov/bridge. It is also available on paper to the U.S. Department of Energy and its contractors, for a processing fee, from:

U.S. Department of Energy

Office of Scientific and Technical Information

P.O. Box 62

Oak Ridge, TN 37831-0062

phone (865) 576-8401

fax (865) 576-5728

reports@adonis.osti.gov

\section{Disclaimer}

This report was prepared as an account of work sponsored by an agency of the United States Government. Reference herein to any specific commercial product, process, or service by trade name, trademark, manufacturer, or otherwise, does not necessarily constitute or imply its endorsement, recommendation, or favoring by the United States Government or any agency thereof. The views and opinions of document authors expressed herein do not necessarily state or reflect those of the United States Government or any agency thereof, Argonne National Laboratory, or UChicago Argonne, LLC. 


\section{Annual Report of Groundwater Monitoring at Everest, Kansas, in 2013}

by

Applied Geosciences and Environmental Management Section

Environmental Science Division, Argonne National Laboratory

February 2014

USDA 


\section{Contents}

Notation.

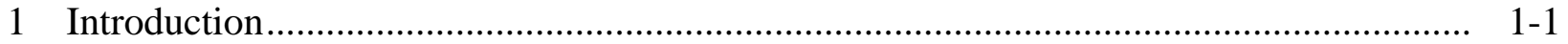

2 Sampling and Analysis Activities................................................................... 2-1

2.1 Measurement of Groundwater Levels.............................................................. 2-1

2.2 Groundwater and Surface Water Sampling and Analysis......................................... 2-1

2.3 Vegetation Sampling and Analysis ........................................................................... 2-2

2.4 Handling and Disposal of Investigation-Derived Waste ....................................... 2-3

2.5 Quality Control for Sample Collection, Handling, and Analysis ........................... 2-3

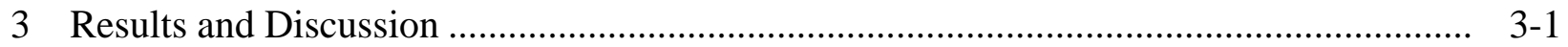

3.1 Groundwater Level Data.............................................................................. 3-1

3.2 Groundwater Analysis Results.......................................................................... 3-1

3.3 Surface Water Analysis Results .................................................................... 3-2

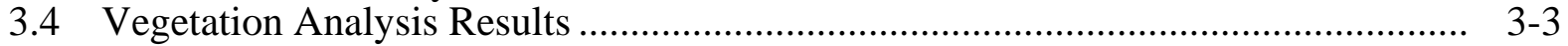

4 Conclusions and Recommendations ................................................................. $4-1$

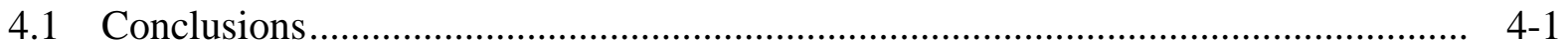

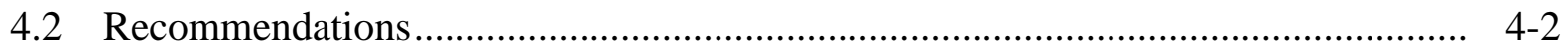

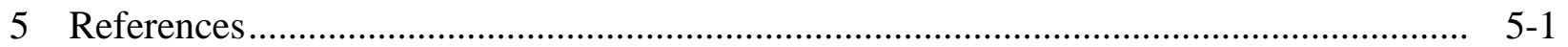

Appendix A: $\quad$ Sequence of Sampling Activities during the 2013 Annual Monitoring Event at Everest ....................................................................... A -1

Appendix B: $\quad$ Quality Control Data Summary …..................................................... B-1

Supplement 1: Groundwater Verification Data from TestAmerica Laboratories, Inc......... on CD

\section{Figures}

$1.1 \quad$ Location of Everest, Kansas ........................................................................ $1-6$

2.1 Groundwater sampling locations in May 2013 .................................................. 2-5 
2.2 Surface water sampling locations in May 2013 ............................................................ 2-6

$2.3 \quad$ Vegetation sampling locations in September 2013.................................................... 2-7

3.1 Potentiometric surface interpreted from groundwater levels measured on May 8-9, 2013

3.2 Historical potentiometric surface interpretations from groundwater levels measured in 2009, 2010, 2011, and 2012

3.3 Lateral distribution of carbon tetrachloride in groundwater in May 2013.

3.4 Lateral distribution of chloroform in groundwater in May 2013.

3.5 Interpreted carbon tetrachloride contaminant plume in groundwater at Everest, for data collected in May 2013

3.6 Historical interpretations of the carbon tetrachloride contaminant plume in groundwater at Everest, for data collected in (a) 2001-2002 and 2006, (b) 2008 and 2010, (c) 2011 and 2012, and (d) 2001-2002 and 2013.

\section{Tables}

1.1 Summary of environmental investigation reports for Everest, Kansas......................... 1-5

3.1 Hand-measured water levels during annual monitoring in 2009-2013......................... 3-4

3.2 Analytical results for VOCs in groundwater samples collected in 2001-2013 at monitoring wells in the approved monitoring network.

3.3 Field measurements for groundwater samples collected in 2001-2013 at monitoring locations in the approved monitoring network.

3.4 Analytical results for VOCs in surface water samples collected during annual monitoring in 2009-2013

3.5 Analytical results for VOCs in tree branch samples collected on September 24, 2013 .

3.6 Analytical results for tree branch samples collected in 2006-2013 3-15

A.1 Sequence of sampling activities during annual monitoring in 2013 
Everest, Kansas, Monitoring in 2013

Version 01, 11/05/13

B.1 Analytical results from the AGEM Laboratory for quality control samples collected during annual monitoring in 2013

B.2 Analytical results for verification groundwater samples collected during annual monitoring in 2013. 


\section{Notation}

AGEM Applied Geosciences and Environmental Management

AMSL above mean sea level

${ }^{\circ} \mathrm{C} \quad$ degree(s) Celsius

CCC Commodity Credit Corporation

CD compact disc

COC chain of custody

DO dissolved oxygen

EPA U.S. Environmental Protection Agency

$\mathrm{ft} \quad$ foot (feet)

gpm gallon(s) per minute

in. inch(es)

KDHE Kansas Department of Health and Environment

L liter(s)

$\mu \mathrm{g} / \mathrm{kg} \quad$ microgram(s) per kilogram

$\mu \mathrm{g} / \mathrm{L} \quad$ microgram(s) per liter

$\mu \mathrm{S} / \mathrm{cm} \quad$ microsiemen(s) per centimeter

$\mathrm{mg} / \mathrm{L} \quad$ milligram(s) per liter

mi mile(s)

$\mathrm{mL} \quad$ milliliter(s)

$\mathrm{mV} \quad$ millivolt(s)

ORP oxidation-reduction potential

TOC top of casing

USDA U.S. Department of Agriculture

VOC volatile organic compound

yr year(s) 


\section{Annual Report of Groundwater Monitoring at Everest, Kansas, in 2013}

\section{Introduction}

Everest, Kansas, is a small rural community (population approximately 300) located in the southeast corner of Brown County, in the northeastern corner of Kansas (Figure 1.1). Everest lies on State Highway 20, approximately $6 \mathrm{mi}$ east of Horton (population 1,885) and $12 \mathrm{mi}$ south of Hiawatha (population 3,603), the county seat. Atchison (population 10,656) is about $23 \mathrm{mi}$ southeast of Everest. Everest is located in Section 29, Township 4 South, Range 18 East, at the approximate latitude $39^{\circ} 40^{\prime} 37^{\prime \prime}$ North and longitude $95^{\circ} 25^{\prime} 20^{\prime \prime}$ West (USGS 1979).

Carbon tetrachloride and chloroform contamination in groundwater at Everest was initially identified in 1997 as a result of testing performed under the Commodity Credit Corporation/U.S. Department of Agriculture (CCC/USDA) private well sampling program conducted by the Kansas Department of Health and Environment (KDHE). The KDHE collected samples from seven private wells in and near Everest. Carbon tetrachloride and chloroform were found in only one of the wells, the Donnie Nigh domestic well (owned at that time by Tim Gale), approximately $3 / 8 \mathrm{mi}$ northwest of the former Everest CCC/USDA facility. Carbon tetrachloride and chloroform were detected at $121 \mu \mathrm{g} / \mathrm{L}$ and $4 \mu \mathrm{g} / \mathrm{L}$, respectively. Nitrate was found at $12.62 \mathrm{mg} / \mathrm{L}$. The USDA subsequently connected the Nigh residence to the Everest public water supply system.

Prior to 1986, commercial grain fumigants containing carbon tetrachloride were commonly used by the CCC/USDA, as well as private and commercial grain storage operations, to preserve grain. In 1997-1998, the KDHE (1998a,b) conducted preliminary investigations to determine potential sources of the identified carbon tetrachloride contamination at Everest. Potential sources included the former CCC/USDA grain storage facility in the western portion of Everest, the Collins Fertilizer and Chemical Company (formerly EDCO Grain, Inc.) in the central portion of town, and a former CCC/USDA grain storage facility located approximately $0.5 \mathrm{mi}$ northeast of town. In addition, historical aerial photos showed grain storage structures on the Nigh (formerly Gale) property.

Because the identified carbon tetrachloride contamination could, in part, be linked to historical use of carbon tetrachloride-based fumigants at its former facility, in 2000 the CCC/USDA assumed responsibility for investigation of the carbon tetrachloride contamination at 
Everest. The CCC/USDA involvement began with development and implementation of a work plan for a Phase I site characterization (Argonne 2000). That investigation and subsequent investigations (Argonne 2001, 2003, 2006a,d) were performed for the CCC/USDA by the Environmental Science Division of Argonne National Laboratory. The results of the environmental investigations at Everest have been reported in detail previously (Table 1.1).

The key results and findings of prior investigations at Everest indicated the following:

1. Concentrations of carbon tetrachloride and chloroform in both near-surface and subsurface vadose zone soils at the former Everest CCC/USDA facility were below KDHE risk-based screening levels, indicating that no source of carbon tetrachloride contamination remained at the former CCC/USDA grain storage facility.

2. Analyses of near-surface soil samples and vegetation samples on the downgradient Nigh (formerly Gale) property indicated a source of carbon tetrachloride contamination to the groundwater associated with private grain storage bins formerly located on that property, as identified in historical aerial photos.

3. The groundwater plume of carbon tetrachloride at concentrations in excess of the maximum contaminant level of $5.0 \mu \mathrm{g} / \mathrm{L}$ was determined to extend downgradient from the former CCC/USDA facility. Low contaminant levels near the former CCC/USDA grain storage facility were consistent with past release of carbon tetrachloride-based grain fumigants at the former CCC/USDA facility. Higher contaminant levels at the Nigh (formerly Gale) property were consistent with the source area associated with the identified grain storage at that property. Both the monitoring data for carbon tetrachloride and the low groundwater flow rates estimated for the Everest aquifer unit (Argonne 2003, 2006a,d, 2008, 2010a, 2011a,b, 2013) indicated slow contaminant migration.

4. Contaminant migration in the aquifer unit was determined to occur via a complex network of generally saturated, discontinuous sandy channels, 
stringers, and lenses in the relatively less permeable and variably saturated sandy clay till.

5. The results of test pumping $(<1.5 \mathrm{gpm}$ sustainable) and hydraulic conductivity estimates obtained from grain size analyses, aquifer pump testing, and slug testing (generally $<3 \mathrm{ft} /$ day) indicated that pumping to actively withdraw groundwater was not viable across most of the contaminant plume. The KDHE concurred that groundwater extraction is not a viable treatment method and encouraged the CCC/USDA to evaluate innovative technologies (KDHE 2006).

6. The predicted discharge point of the contaminant plume was determined to be an intermittent stream west of the former CCC/USDA grain storage area and southwest of the Nigh property, limiting the potential for further westward migration of contaminated groundwater.

7. No current domestic wells are impacted or threatened.

8. The Everest municipal water system supplies drinking water to all residents of the town of Everest and to all private residences outside the city limits that are in the general vicinity of the existing groundwater plume.

9. Results of indoor air sampling in August 2010 in 10 homes overlying the identified contaminant plume indicated no potential threat to human health due to indoor air contaminated as a result of activities at the former CCC/USDA facility.

In 2007, two contingency response actions were proposed, should ongoing investigation and monitoring indicate the need. These response actions were (1) implementation of phytoremediation to address the downgradient plume (Argonne 2007a) and (2) installation of additional monitoring wells to address potential changes in plume migration (Argonne 2007b).

In March 2009, the CCC/USDA developed a plan for annual monitoring of the groundwater and surface water (Argonne 2009). Under this plan, approved by the KDHE (2009), monitoring wells are sampled by using the low-flow procedure (Puls and Barcelona 1996; Yeskis 
and Zavala 2002), and surface water samples are collected at five locations along the intermittent creek. Vegetation sampling is conducted as a secondary indicator of plume migration.

Results of annual sampling in 2009-2012 for volatile organic compounds (VOCs) and water level measurements (Argonne 2010a, 2011a,b, 2013) were consistent with previous observations (Argonne 2003, 2006a,d, 2008). No carbon tetrachloride was detected in surface water of the intermittent creek at locations along the creek banks.

This report presents the results of the annual sampling event conducted in 2013. Included in the 2013 monitoring were the following:

- $\quad$ Surface water sampling on May 8, 2013.

- Groundwater sampling on May 8-9, 2013.

- Vegetation sampling on September 24, 2013.

The activities are described in Section 2, and the results are discussed in Section 3. Conclusions and recommendations are presented in Section 4. 
TABLE 1.1 Summary of environmental investigation reports for Everest, Kansas.

\begin{tabular}{|c|c|}
\hline Report & Reference \\
\hline \multicolumn{2}{|l|}{ KDHE reports } \\
\hline Site reconnaissance and evaluation for the Everest CCC/USDA site & KDHE 1998a \\
\hline Site reconnaissance and evaluation for the Everest East CCC/USDA site & KDHE 1998b \\
\hline \multicolumn{2}{|l|}{ CCC/USDA reports } \\
\hline Work plan for Phase I investigation & Argonne 2000 \\
\hline Phase I report and Phase II work plan & Argonne 2001 \\
\hline Phase II report & Argonne 2003 \\
\hline Scoping memo of options for restoration of the Everest aquifer unit & Argonne 2005 \\
\hline Phase III targeted investigation report & Argonne 2006a \\
\hline Cross section analysis and recommendations for further studies & Argonne 2006b \\
\hline Plan for aquifer hydraulic testing and groundwater sampling in January-February 2006 & Argonne $2006 \mathrm{c}$ \\
\hline Results of aquifer pumping and groundwater sampling in January-March 2006 & Argonne 2006d \\
\hline Recommendations for new monitoring wells & Argonne $2007 a$ \\
\hline Recommendations for remedial action & Argonne $2007 b$ \\
\hline Results of groundwater monitoring in April 2008 & Argonne 2008 \\
\hline Monitoring plan & Argonne 2009 \\
\hline Results of groundwater monitoring and vegetation sampling in 2009 & Argonne $2010 a$ \\
\hline Work plan for indoor air sampling & Argonne $2010 b$ \\
\hline Results of groundwater monitoring in 2010 & Argonne $2011 \mathrm{a}$ \\
\hline Results of groundwater monitoring in 2011 & Argonne 2011b \\
\hline Results of groundwater monitoring in 2012 & Argonne 2013 \\
\hline
\end{tabular}




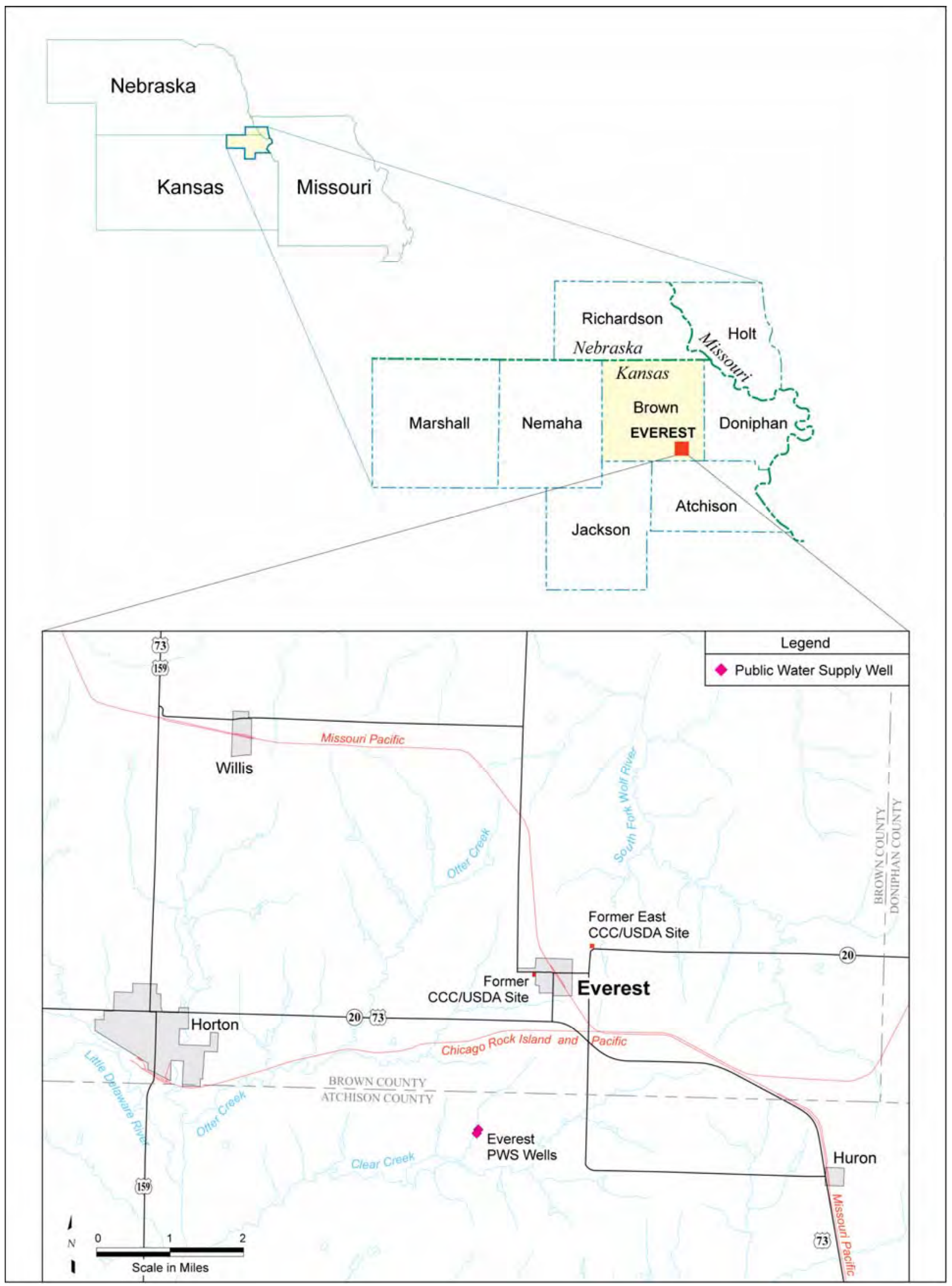

FIGURE 1.1 Location of Everest, Kansas. 


\section{Sampling and Analysis Activities}

\subsection{Measurement of Groundwater Levels}

Water level sensors incorporating data loggers were used in 2002-2010 for automatic, continuous recording of groundwater levels at numerous locations. Hydrographs generated from the recorder data indicated little net change in the groundwater levels during the period of record. The hydrographs depicted a consistent, seasonal pattern of (1) rising groundwater levels in association with spring and early summer rains, (2) falling groundwater levels during the later summer and early fall, and (3) relatively stable groundwater elevations during the winter and early spring. The accumulated data demonstrated that the relative impact of the spring and early summer recharge events to the aquifer unit is greatest in the eastern portion of the study area. This effect decreases progressively at locations west of the Nigh property, toward the eventual (potential) intermittent creek discharge area (Argonne 2011a).

The automatic and manual groundwater level measurements made in 2002-2010 demonstrated a consistent groundwater flow pattern, with only transient seasonal variations in the magnitude (but not the direction) of the hydraulic gradients. As a result, automatic water level monitoring at Everest was suspended. After a final data download on October 1, 2010, the recorders were removed (Argonne 2011a). Water level determinations are currently based on hand measurements made at the time of the annual groundwater sampling.

During the present reporting period, manual water level measurements were made in the wells sampled for VOCs during the annual groundwater sampling event on May 8-9, 2013. The manual groundwater level measurements were made by using a water level indicator to determine the depth to groundwater, within $0.01 \mathrm{ft}$, from the top of the well casing (TOC).

The groundwater level data from the annual monitoring events in 2009-2013 are presented and discussed in Section 3.1.

\subsection{Groundwater and Surface Water Sampling and Analysis}

On May 8-9, 2013, groundwater samples were collected for VOCs analyses at 14 locations (MW1-MW4, SB09, SB34, SB49, SB60, SB62-SB64, SB78, SB80, SB88) along 
the identified contaminant migration pathway (Figure 2.1). Samples were collected from the groundwater monitoring points by using the low-flow procedure (Puls and Barcelona 1996; Yeskis and Zavala 2002). On May 8, 2013, surface water samples were collected for VOCs analyses at 5 locations (Figure 2.2) along the intermittent creek to the west (downgradient) of the identified carbon tetrachloride contamination in groundwater. Surface water samples were collected for VOCs analyses by dipping a laboratory-approved container beneath the ambient water surface at each required sampling location. The sequence of groundwater and surface water sampling activities during the 2013 monitoring event is summarized in Appendix A, Table A.1.

The groundwater and surface water samples were labeled, packaged, and chilled to $4^{\circ} \mathrm{C}$ by placement in ice-filled coolers. The samples were shipped overnight to the Applied Geosciences and Environmental Management (AGEM) Laboratory at Argonne for VOCs analyses with U.S. Environmental Protection Agency (EPA) Method 524.2 (EPA 1995). Aliquots of selected samples (chosen in the field) were also shipped directly to TestAmerica Laboratories, Inc., South Burlington, Vermont, for verification VOCs analyses according to EPA Contract Laboratory Program protocols.

The results of the groundwater and surface water analyses are presented in Sections 3.2 and 3.3, respectively.

\subsection{Vegetation Sampling and Analysis}

Argonne experience has demonstrated that the sampling and analysis of native vegetation for carbon tetrachloride, and particularly tree branch tissues, provides a sensitive indicator of carbon tetrachloride contamination in the shallow groundwater in the plant rooting zone. Annual monitoring at Everest has therefore included sampling of existing vegetation along the downgradient path of the identified contaminant plume. As in prior annual monitoring events, samples of branch tissues from tree species including elm, pin cherry, mulberry, Osage orange, willow, and cottonwood were collected on September 24, 2013, at locations along the intermittent creek and upgradient in the source area identified on the Nigh (formerly Gale) property (Figure 2.3). The branch samples were cut and sealed in 40-mL glass vials, then placed immediately on dry ice for shipment to the AGEM Laboratory for analysis (Alvarado and Rose 2004). 
Of the 20 vegetation locations sampled in 2013 (Figure 2.3), 14 had been sampled since 2006-2007. In two of these cases (EV008/EV008A and EV013/EV013A), the original tree has died, and an adjacent tree is now sampled instead. The other 6 locations sampled in 2013 were new locations in 2011 (EV026, EV027, and EV028 along the creek, as well as EV029, EV030, and EV031 on the Nigh property). Location EV032 (new in 2012) could not be sampled in 2013, because the tree has been removed. The EV032 location is now an agricultural field.

\subsection{Handling and Disposal of Investigation-Derived Waste}

Purge water generated as potentially contaminated investigation-derived waste during the May 2013 Everest monitoring was containerized on-site. The volume of purge water accumulated totaled approximately $49 \mathrm{~L}$. Because of the very low volume, the accumulated wastewater remains on-site pending completion of required monitoring at other Kansas sites. At that time, the wastewater from all monitoring sites will be sampled and disposed of as appropriate. During past annual monitoring events, analysis of wastewater was conducted at Pace Analytical Services, Inc., Lenexa, Kansas, by EPA Methods 5030/8260 for VOCs, EPA Method 504.1 for ethylene dibromide, and EPA Method 353.2 for nitrate/nitrite nitrogen. Disposal of wastewater has historically been at the Sabetha publicly owned treatment works.

\subsection{Quality Control for Sample Collection, Handling, and Analysis}

The quality assurance/quality control procedures followed during the 2013 annual monitoring for collection, handling, and analysis of samples are described in detail in the Master Work Plan (Argonne 2002). Results of these activities are summarized as follows:

- Sample collection and handling activities were monitored by the documentation of samples as they were collected and the use of chain-ofcustody forms and custody seals to ensure sample integrity during handling and shipment.

- Samples designated for VOCs analyses were received with custody seals intact and at the appropriate preservation temperature. All samples were analyzed within the required holding times. 
- Quality control samples collected to monitor sample-handling activities (two field blanks, two equipment rinsates, and one trip blank; Table B.1 in Appendix B) and method blanks analyzed with the samples to monitor analytical methodologies were all free of carbon tetrachloride and chloroform contamination.

- Groundwater and surface water samples were analyzed for VOCs at the AGEM Laboratory by the purge-and-trap method on a gas chromatographmass spectrometer system. Calibration checks analyzed with each sample delivery group were required to be within $\pm 20 \%$ of the standard. Surrogate standard determinations performed on samples and blanks were within the specified range of $80-120 \%$ for all samples, in either the initial analysis or a successful reanalysis.

- Accuracy and precision of the analytical methodology were evident in the analysis of two replicate samples and the duplicate analysis of seven samples, with average relative percent difference values between the initial analyses and the associated quality control analyses of $<4 \%$ and $<2 \%$ for carbon tetrachloride and chloroform, respectively (Table B.1 in Appendix B). The analytical data from the AGEM Laboratory for groundwater and surface water are acceptable for quantitative determination of contaminant distribution.

- In accordance with the procedures defined in the Master Work Plan (Argonne 2002), selected samples were submitted to a second laboratory (TestAmerica) for verification analysis according to the protocols of the EPA's Contract Laboratory Program. The results from the two laboratories compare favorably over the range of contaminant concentrations detected, with average relative percent difference values of $<7 \%$ and $<4 \%$ for carbon tetrachloride and chloroform, respectively (Table B.2 in Appendix B). The analytical data provided by TestAmerica for sample delivery group 200-16407 are in Supplement 1 (on the compact disc [CD] inside the back cover of this report). 


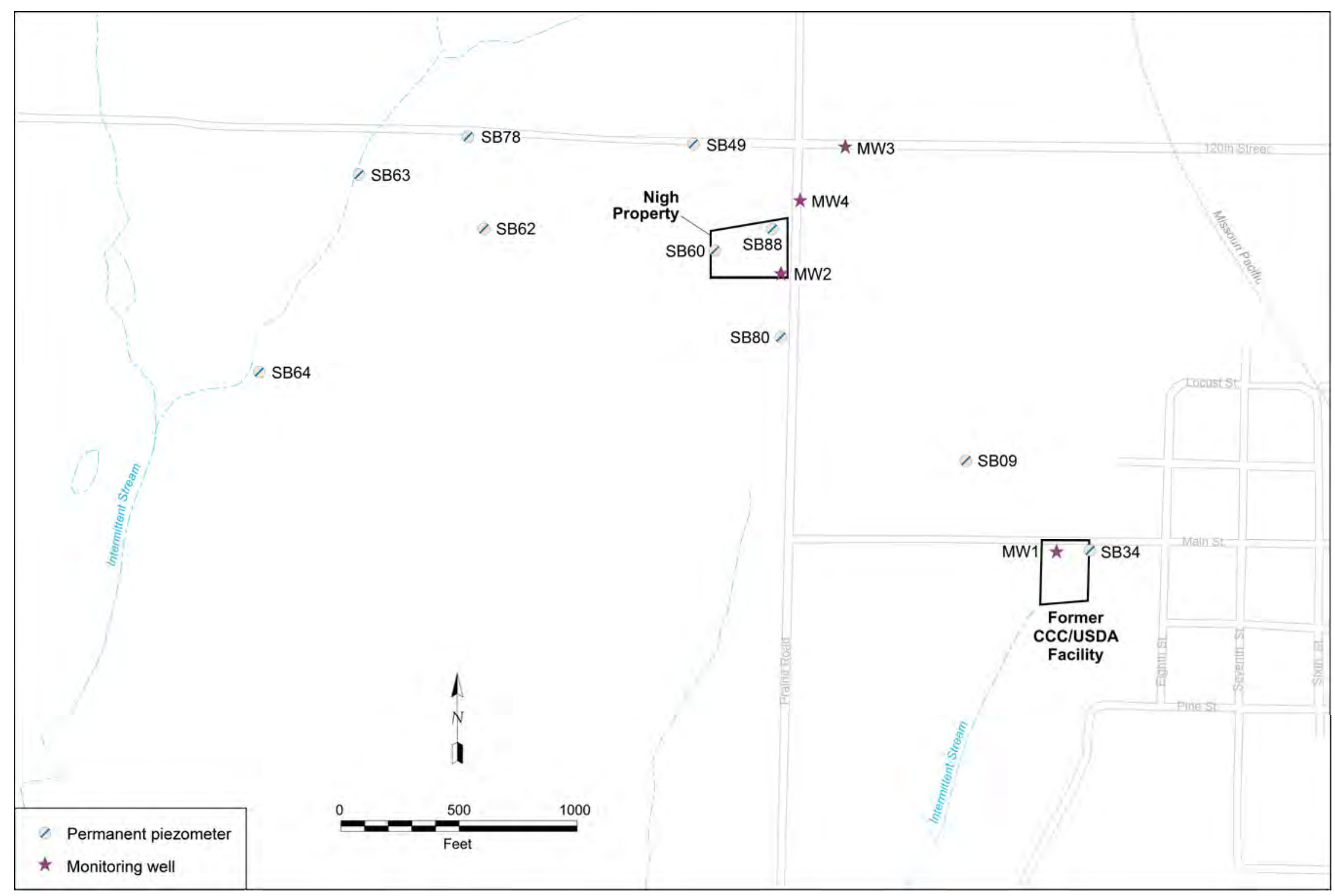

FIGURE 2.1 Groundwater sampling locations in May 2013. 


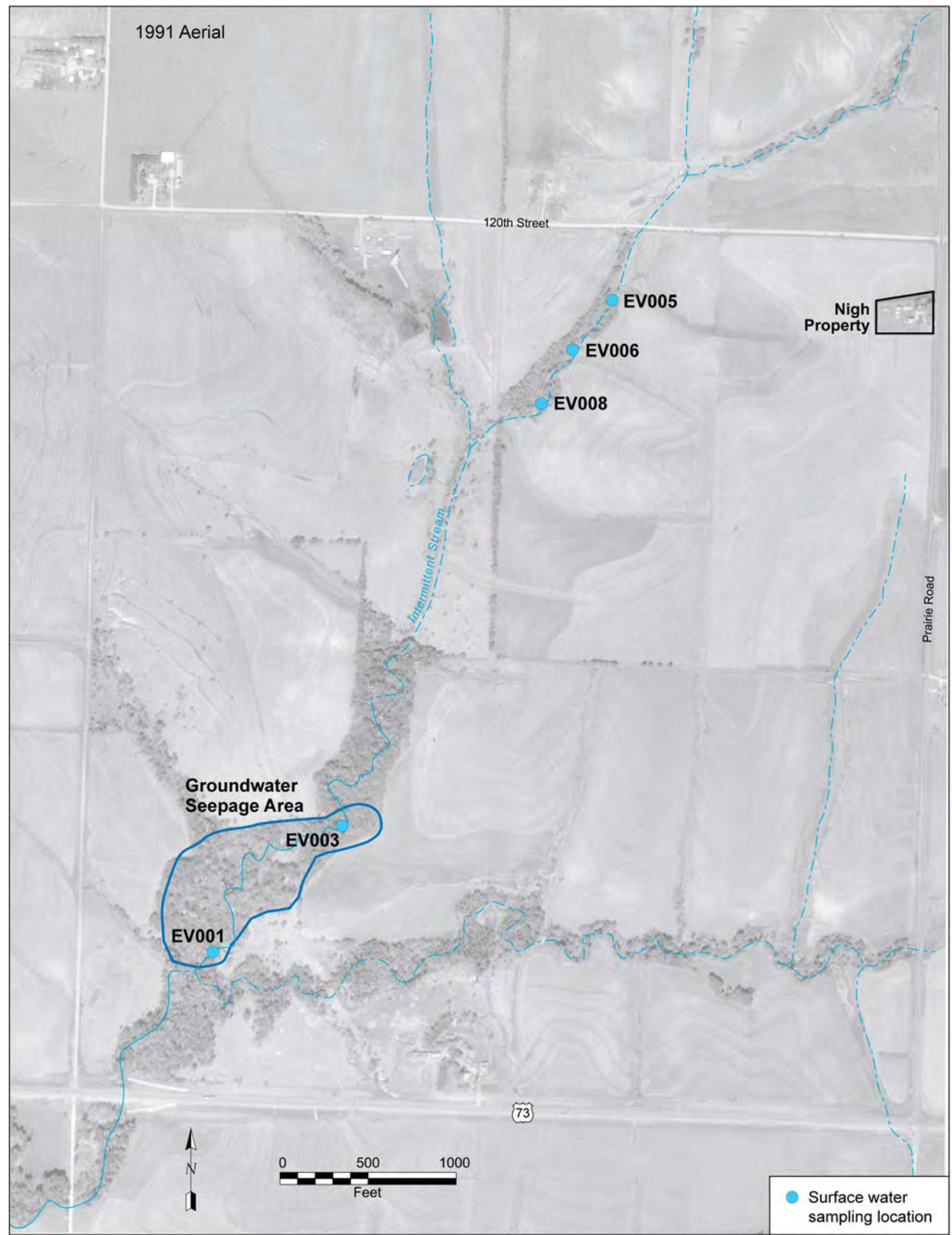

FIGURE 2.2 Surface water sampling locations in May 2013. 


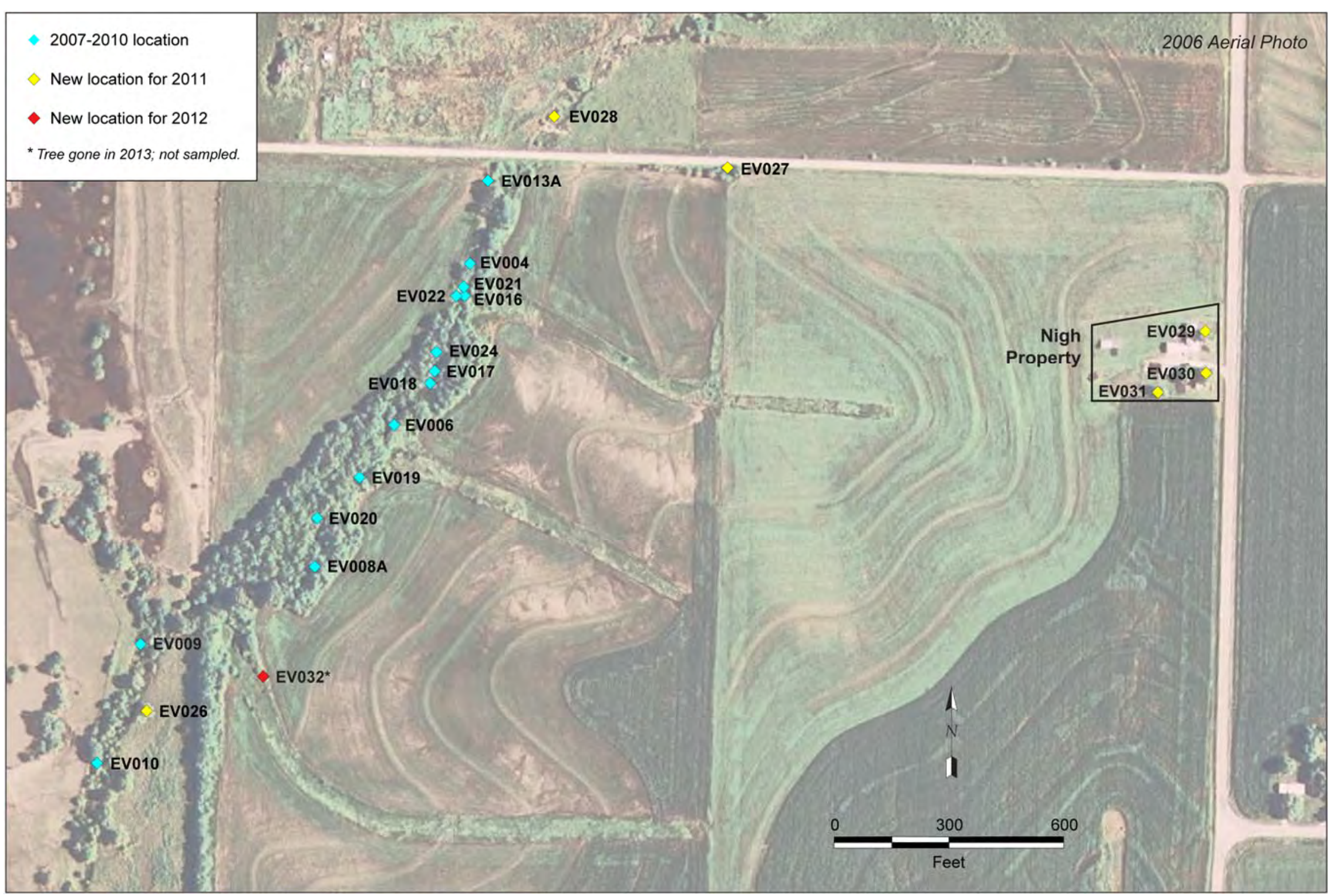

FIGURE 2.3 Vegetation sampling locations in September 2013. 


\section{Results and Discussion}

\subsection{Groundwater Level Data}

Argonne's investigations (Argonne 2003, 2006a,d) determined that groundwater flow in the aquifer unit of concern occurs at a relatively low rate, in association with estimated hydraulic conductivities for this interval that are generally $<3 \mathrm{ft} /$ day. The flow is driven primarily by groundwater recharge to the southeast of the former CCC/USDA facility and by discharge along the lower (perennial) reaches of the intermittent creek southwest of the Nigh (formerly Gale) property. The result is a relatively stable pattern of groundwater flow across the investigation area. The detailed pathways of groundwater flow and contaminant migration are influenced, however, by hydrogeologic heterogeneity in the aquifer unit, particularly by a region of reduced sand content and hence decreased permeability near the Nigh property.

Figure 3.1 shows the configuration of the potentiometric surface at the site, as determined from manual groundwater level measurements on May 8-9, 2013. For comparison, Figure 3.2 shows analogous mapping based on 2009, 2010, 2011, and 2012 measurements. Together, Figure 3.1 and Figure 3.2 demonstrate that the potentiometric surface - and hence the groundwater flow pattern influencing contaminant distribution - has remained consistent with the above interpretation.

The hand-measured water level data from annual monitoring in 2009-2013 are in Table 3.1.

\subsection{Groundwater Analysis Results}

The analytical data for VOCs and the field-measured parameters for the groundwater samples collected on May 8-9, 2013, are in Tables 3.2 and 3.3, respectively, together with previous data for these wells. The May 2013 data for carbon tetrachloride and chloroform are illustrated in Figures 3.3 and 3.4, respectively.

Figure 3.5 illustrates the configuration of the carbon tetrachloride plume, as interpreted from the 2013 data. Over the years of investigation, the plume has been interpreted as shown in Figure 3.6. The pattern of groundwater flow and contaminant migration from the former 
CCC/USDA facility has consistently been to the north-northwest toward the Nigh (formerly Gale) property, then west-southwest from the Nigh property toward the intermittent creek, as shown in Figures 3.1 and 3.2.

The comparison in Figure 3.6 (and Table 3.2) demonstrates that the area of the plume with carbon tetrachloride concentrations $>200 \mu \mathrm{g} / \mathrm{L}$ has decreased markedly over time and suggests a generally decreasing trend in contaminant levels. Noteworthy decreases approaching and exceeding 50\% (from previous peak values) have occurred at locations MW4 and SB88, in the most concentrated part of the plume. During investigation/monitoring since 2000, the plume has shown little or no definitive expansion toward the intermittent creek over time (Argonne 2003, 2006a,d, 2008, 2010a, 2011a,b, 2013). This observation is consistent with very slow groundwater movement and contaminant migration.

Table 3.3 indicates some fluctuation, but no consistent trends, in dissolved oxygen (DO) levels and oxidation-reduction potential (ORP) values over the period of record. The associated $\mathrm{pH}$ and conductivity measurements indicate a relatively stable geochemical environment in the aquifer.

\subsection{Surface Water Analysis Results}

The accumulated investigation data since 2000 demonstrate little migration of the diminishing carbon tetrachloride plume toward the intermittent creek west of the former CCC/USDA facility and the Nigh property.

The results of VOCs analysis of the surface water samples collected on May 8, 2013, are in Table 3.4, along with the results from prior annual monitoring in 2009-2012. As shown, neither carbon tetrachloride nor chloroform has been detected at the purge-and-trap method detection limit at any of the surface water sampling locations (Figure 2.2) along the intermittent creek downgradient from the identified groundwater plume, indicating no impact to the surface water pathway. 


\subsection{Vegetation Analysis Results}

Table 3.5 summarizes the results of VOCs analyses of vegetation samples collected on September 24, 2013, along the creek and in the source area at the former private grain storage area on the Nigh property. No carbon tetrachloride was detected in these samples. The present data are consistent with the historical results of vegetation analyses (Table 3.6). The 2013 data do not corroborate the trace levels of carbon tetrachloride identified at some vegetation sampling locations during the July 2012 sampling event (Table 3.6). The predominant lack of carbon tetrachloride detections over time in the vegetation along the creek is consistent with the interpreted slow migration of groundwater and contamination toward the downgradient intermittent stream. 
TABLE 3.1 Hand-measured water levels during annual monitoring in 2009-2013.

\begin{tabular}{|c|c|c|c|c|c|c|c|c|c|c|c|}
\hline \multirow[b]{2}{*}{ Well } & \multirow[b]{2}{*}{$\begin{array}{l}\text { Reference } \\
\text { Elevation } \\
\text { (ft AMSL) }\end{array}$} & \multicolumn{2}{|c|}{ April 25-26, 2009} & \multicolumn{2}{|c|}{ April 8-9, 2010} & \multicolumn{2}{|c|}{ April 27-28, 2011} & \multicolumn{2}{|c|}{ April 3-4, 2012} & \multicolumn{2}{|c|}{ May 8-9, 2013} \\
\hline & & $\begin{array}{l}\text { Depth to } \\
\text { Water } \\
\text { (ft TOC) }\end{array}$ & $\begin{array}{c}\text { Water } \\
\text { Elevation } \\
\text { (ft AMSL) }\end{array}$ & $\begin{array}{l}\text { Depth to } \\
\text { Water } \\
\text { (ft TOC) }\end{array}$ & $\begin{array}{c}\text { Water } \\
\text { Elevation } \\
\text { (ft AMSL) }\end{array}$ & $\begin{array}{l}\text { Depth to } \\
\text { Water } \\
\text { (ft TOC) }\end{array}$ & $\begin{array}{c}\text { Water } \\
\text { Elevation } \\
\text { (ft AMSL) }\end{array}$ & $\begin{array}{l}\text { Depth to } \\
\text { Water } \\
\text { (ft TOC) }\end{array}$ & $\begin{array}{c}\text { Water } \\
\text { Elevation } \\
\text { (ft AMSL) }\end{array}$ & $\begin{array}{l}\text { Depth to } \\
\text { Water } \\
\text { (ft TOC) }\end{array}$ & $\begin{array}{c}\text { Water } \\
\text { Elevation } \\
\text { (ft AMSL) }\end{array}$ \\
\hline SB09 & 1138.94 & 31.50 & 1107.44 & 19.70 & 1119.24 & 23.93 & 1115.01 & 19.21 & 1119.73 & 27.32 & 1111.62 \\
\hline SB34 & 1131.73 & 9.50 & 1122.23 & 9.16 & 1122.57 & 13.28 & 1118.45 & 9.35 & 1122.38 & 14.44 & 1117.29 \\
\hline SB49 & 1132.48 & 37.40 & 1095.08 & 36.65 & 1095.83 & 40.88 & 1091.60 & 37.60 & 1094.88 & 41.48 & 1091.00 \\
\hline SB60 & 1144.11 & 48.20 & 1095.91 & 47.70 & 1096.41 & 51.44 & 1092.67 & 47.85 & 1096.26 & 52.86 & 1091.25 \\
\hline SB62 & 1121.22 & 29.00 & 1092.22 & 25.60 & 1095.62 & 31.51 & 1089.71 & 28.32 & 1092.90 & 32.45 & 1088.77 \\
\hline SB63 & 1104.75 & 19.20 & 1085.55 & 16.71 & 1088.04 & 18.87 & 1085.88 & 17.20 & 1087.55 & 20.57 & 1084.18 \\
\hline SB64 & 1098.36 & 21.20 & 1077.16 & 17.30 & 1081.06 & 20.02 & 1078.34 & 20.25 & 1078.11 & 20.67 & 1077.69 \\
\hline SB72 & 1112.53 & 28.20 & 1084.33 & 27.10 & 1085.43 & - & - & - & $\begin{array}{c}0 . \perp+ \\
-\end{array}$ & - & - \\
\hline SB77 & 1124.57 & 41.00 & 1083.57 & 40.25 & 1084.32 & - & - & - & - & - & - \\
\hline SB78 & 1118.22 & 24.30 & 1093.92 & 25.00 & 1093.22 & 28.38 & 1089.84 & 23.90 & 1094.32 & 26.36 & 1091.86 \\
\hline SB80 & 1149.72 & 44.60 & 1105.12 & 42.30 & 1107.42 & 47.82 & 1101.90 & 43.55 & 1106.17 & 49.71 & 1100.01 \\
\hline SB88 & 1151.02 & 45.20 & 1105.82 & 44.10 & 1106.92 & 51.82 & 1099.20 & 44.58 & 1106.44 & 55.00 & 1096.02 \\
\hline MW1 & 1127.08 & 4.80 & 1122.28 & 4.50 & 1122.58 & 8.83 & 1118.25 & 5.05 & 1122.03 & 9.93 & 1117.15 \\
\hline MW2 & 1151.68 & 47.50 & 1104.18 & 46.40 & 1105.28 & 51.69 & 1099.99 & 47.75 & 1103.93 & 54.50 & 1097.18 \\
\hline MW3 & 1144.92 & 31.50 & 1113.42 & 30.60 & 1114.32 & 39.72 & 1105.20 & 33.50 & 1111.42 & 40.48 & 1104.44 \\
\hline MW4 & 1148.93 & - & - & 36.20 & 1112.73 & 43.46 & 1105.47 & 39.05 & 1109.88 & 46.68 & 1102.25 \\
\hline
\end{tabular}


TABLE 3.2 Analytical results for VOCs in groundwater samples collected in 2001-2013 at monitoring wells in the approved monitoring network. ${ }^{a}$

\begin{tabular}{|c|c|c|c|c|c|c|c|}
\hline \multirow[b]{2}{*}{ Location } & \multirow[b]{2}{*}{ Sample } & \multirow[b]{2}{*}{$\begin{array}{l}\text { Sample } \\
\text { Date }\end{array}$} & \multirow[b]{2}{*}{$\begin{array}{l}\text { Depth } \\
\text { (ft BGL) }\end{array}$} & \multicolumn{4}{|c|}{ Concentration $(\mu \mathrm{g} / \mathrm{L})$} \\
\hline & & & & $\begin{array}{l}\text { Sample } \\
\text { Type }^{b}\end{array}$ & $\begin{array}{c}\text { Carbon } \\
\text { Tetrachloride }\end{array}$ & Chloroform & $\begin{array}{l}\text { Methylene } \\
\text { Chloride }\end{array}$ \\
\hline MW1 & EVMW1-W-20103 & 3/23/2006 & $41-51$ & $\mathrm{~N}$ & 2.0 & $0.4 \mathrm{~J}^{\mathrm{c}}$ & $N D^{d}$ \\
\hline MW1 & EVMW1-W-23441 & 4/1/2008 & $41-51$ & $\mathrm{~N}$ & 1.6 & $0.5 \mathrm{~J}$ & ND \\
\hline MW1 & EVMW1-W-26326 & $4 / 25 / 2009$ & $41-51$ & $\mathrm{~N}$ & 38 & 3.2 & ND \\
\hline MW1 & EVMW1-W-29743 & $4 / 8 / 2010$ & $41-51$ & $\mathrm{~N}$ & 59 & 4.8 & ND \\
\hline MW1 & EVMW1-W-29770 & $4 / 27 / 2011$ & $41-51$ & $\mathrm{~N}$ & 54 & 3.8 & ND \\
\hline MW1 & EVMW1-W-29797 & $4 / 3 / 2012$ & $41-51$ & $\mathrm{~N}$ & 29 & 2.9 & ND \\
\hline MW1 & EVMW1DUP-W-29811 ${ }^{\mathrm{e}}$ & $4 / 3 / 2012$ & $41-51$ & DUP-F & 30 & 3.0 & ND \\
\hline MW1 & EVMW1-W-29823 & $5 / 8 / 2013$ & $41-51$ & $\mathrm{~N}$ & 3.1 & ND & ND \\
\hline MW1 & EVMW1-W-29823DUP & $5 / 8 / 2013$ & $41-51$ & DUP-L & 2.9 & ND & ND \\
\hline MW2 & EVMW2-W-23442 & $4 / 9 / 2008$ & $59-79$ & $\mathrm{~N}$ & 47 & 1.0 & ND \\
\hline MW2 & EVMW2-W-26327 & $4 / 26 / 2009$ & $59-79$ & $\mathrm{~N}$ & 101 & 2.1 & ND \\
\hline MW2 & EVMW2-W-29744 & 4/8/2010 & $59-79$ & $\mathrm{~N}$ & 124 & 3.3 & ND \\
\hline MW2 & EVMW2-W-29744DUP ${ }^{\mathrm{e}}$ & $4 / 8 / 2010$ & $59-79$ & DUP-L & 129 & 3.1 & ND \\
\hline MW2 & EVMW2-W-29771 & $4 / 27 / 2011$ & $59-79$ & $\mathrm{~N}$ & 70 & 2.5 & ND \\
\hline MW2 & EVMW2DUP-W-29784 & $4 / 27 / 2011$ & $59-79$ & DUP-F & 62 & 2.2 & ND \\
\hline MW2 & EVMW2-W-29798 & 4/4/2012 & $59-79$ & $\mathrm{~N}$ & 141 & 4.3 & ND \\
\hline MW2 & EVMW2-W-29824 & $5 / 9 / 2013$ & $59-79$ & $\mathrm{~N}$ & 87 & 2.7 & ND \\
\hline MW2 & EVMW2-W-29824DUP & $5 / 9 / 2013$ & $59-79$ & DUP-L & 86 & 2.6 & ND \\
\hline MW3 & EVMW3-W-23443 & 4/1/2008 & $56.5-71.5$ & $\mathrm{~N}$ & 7.6 & $0.3 \mathrm{~J}$ & ND \\
\hline MW3 & EVMW3-W-26328 & $4 / 25 / 2009$ & $56.5-71.5$ & $\mathrm{~N}$ & 9.1 & $0.4 \mathrm{~J}$ & ND \\
\hline MW3 & EVMW3-W-29745 & $4 / 8 / 2010$ & $56.5-71.5$ & $\mathrm{~N}$ & 13 & $0.5 \mathrm{~J}$ & ND \\
\hline MW3 & EVMW3-W-29772 & $4 / 28 / 2011$ & $56.5-71.5$ & $\mathrm{~N}$ & 11 & $0.4 \mathrm{~J}$ & ND \\
\hline MW3 & EVMW3DUP-W-29785 & $4 / 28 / 2011$ & $56.5-71.5$ & DUP-F & 12 & $0.3 \mathrm{~J}$ & ND \\
\hline MW3 & EVMW3-W-29799 & 4/4/2012 & $56.5-71.5$ & $\mathrm{~N}$ & 17 & $0.6 \mathrm{~J}$ & ND \\
\hline MW3 & EVMW3-W-29825 & $5 / 8 / 2013$ & $56.5-71.5$ & $\mathrm{~N}$ & 17 & ND & ND \\
\hline MW3 & EVMW3DUP-W-29837 & $5 / 8 / 2013$ & $56.5-71.5$ & DUP-F & 18 & ND & ND \\
\hline MW3 & EVMW3DUP-W-29837DUP & $5 / 8 / 2013$ & $56.5-71.5$ & DUP-L & 17 & ND & ND \\
\hline MW4 & EVMW4-W-20117 & 3/23/2006 & $48.5-68.5$ & $\mathrm{~N}$ & 316 & 13 & ND \\
\hline MW4 & EVMW4-W-23444 & $4 / 2 / 2008$ & $48.5-68.5$ & $\mathrm{~N}$ & 244 & 7.9 & ND \\
\hline MW4 & EVMW4-W-29746 & $4 / 9 / 2010$ & $48.5-68.5$ & $\mathrm{~N}$ & 245 & 8.4 & ND \\
\hline MW4 & EVMW4-W-29746DUP & $4 / 9 / 2010$ & $48.5-68.5$ & DUP-L & 253 & 8.8 & ND \\
\hline MW4 & EVMW4-W-29773 & $4 / 28 / 2011$ & $48.5-68.5$ & $\mathrm{~N}$ & 104 & 4.0 & ND \\
\hline MW4 & EVMW4-W-29800 & 4/3/2012 & $48.5-68.5$ & $\mathrm{~N}$ & 140 & 5.4 & ND \\
\hline MW4 & EVMW4-W-29826 & $5 / 8 / 2013$ & $48.5-68.5$ & $\mathrm{~N}$ & 70 & 2.4 & ND \\
\hline
\end{tabular}


TABLE 3.2 (Cont.)

\begin{tabular}{|c|c|c|c|c|c|c|c|}
\hline \multirow[b]{2}{*}{ Location } & \multirow[b]{2}{*}{ Sample } & \multirow[b]{2}{*}{$\begin{array}{l}\text { Sample } \\
\text { Date }\end{array}$} & \multirow[b]{2}{*}{$\begin{array}{l}\text { Depth } \\
\text { (ft BGL) }\end{array}$} & \multicolumn{4}{|c|}{ Concentration $(\mu \mathrm{g} / \mathrm{L})$} \\
\hline & & & & $\begin{array}{l}\text { Sample } \\
\text { Type }^{\mathrm{b}}\end{array}$ & $\begin{array}{c}\text { Carbon } \\
\text { Tetrachloride }\end{array}$ & Chloroform & $\begin{array}{l}\text { Methylene } \\
\text { Chloride }\end{array}$ \\
\hline SB09 & EVSB09-W-20098 & $3 / 22 / 2006$ & $51-57$ & $\mathrm{~N}$ & 90 & 5.1 & ND \\
\hline SB09 & EVSB09-W-23448 & $4 / 9 / 2008$ & $51-57$ & $\mathrm{~N}$ & 51 & 2.3 & ND \\
\hline SB09 & EVSB09-W-26330 & $4 / 26 / 2009$ & $51-57$ & $\mathrm{~N}$ & 114 & 5.4 & ND \\
\hline SB09 & EVSB09-W-29747 & 4/9/2010 & $51-57$ & $\mathrm{~N}$ & 72 & 4.9 & ND \\
\hline SB09 & EVSB09-W-29747DUP & $4 / 9 / 2010$ & $51-57$ & DUP-L & 75 & 5.3 & ND \\
\hline SB09 & EVSB09DUP-W-29760 & $4 / 9 / 2010$ & $51-57$ & DUP-F & 63 & 4.5 & ND \\
\hline SB09 & EVSB09-W-29774 & $4 / 27 / 2011$ & $51-57$ & $\mathrm{~N}$ & 49 & 3.9 & ND \\
\hline SB09 & EVSB09-W-29801 & 4/4/2012 & $51-57$ & $\mathrm{~N}$ & 56 & 4.3 & ND \\
\hline SB09 & EVSB09-W-29827 & $5 / 9 / 2013$ & $51-57$ & $\mathrm{~N}$ & 39 & 3.2 & ND \\
\hline SB34 & EVSB34-W-12857 & $3 / 28 / 2001$ & $46-49$ & $\mathrm{~N}$ & 2.2 & 1.3 & ND \\
\hline SB34 & EVSB34-W-12854 & $3 / 28 / 2001$ & $49-53$ & $\mathrm{~N}$ & ND & ND & ND \\
\hline SB34 & EVSB34-W-20097 & $3 / 22 / 2006$ & $46-53$ & $\mathrm{~N}$ & 29 & 8.6 & ND \\
\hline SB34 & EVSB34-W-23451 & $4 / 9 / 2008$ & $46-53$ & $\mathrm{~N}$ & 3.2 & 1.1 & ND \\
\hline SB34 & EVSB34-W-26331 & $4 / 26 / 2009$ & $46-53$ & $\mathrm{~N}$ & 4.4 & 1.9 & ND \\
\hline SB34 & EVSB34-W-29748 & 4/9/2010 & $46-53$ & $\mathrm{~N}$ & 2.7 & $0.5 \mathrm{~J}$ & ND \\
\hline SB34 & EVSB34-W-29775 & 4/27/2011 & $46-53$ & $\mathrm{~N}$ & 1.1 & $0.7 \mathrm{~J}$ & ND \\
\hline SB34 & EVSB34-W-29802 & 4/4/2012 & $46-53$ & $\mathrm{~N}$ & $0.7 \mathrm{~J}$ & $0.4 \mathrm{~J}$ & ND \\
\hline SB34 & EVSB34-W-29828 & $5 / 8 / 2013$ & $46-53$ & $\mathrm{~N}$ & 8.4 & 2.0 & ND \\
\hline SB49 & EVSB49-W-20095 & $3 / 22 / 2006$ & $51-55$ & $\mathrm{~N}$ & $0.9 \mathrm{~J}$ & ND & ND \\
\hline SB49 & EVSB49-W-23452 & $4 / 8 / 2008$ & $51-55$ & $\mathrm{~N}$ & 2.1 & ND & ND \\
\hline SB49 & EVSB49-W-26332 & $4 / 26 / 2009$ & $51-55$ & $\mathrm{~N}$ & 3.2 & ND & ND \\
\hline SB49 & EVSB49-W-29749 & $4 / 8 / 2010$ & $51-55$ & $\mathrm{~N}$ & 3.3 & ND & ND \\
\hline SB49 & EVSB49-W-29776 & $4 / 28 / 2011$ & $51-55$ & $\mathrm{~N}$ & 3.0 & ND & ND \\
\hline SB49 & EVSB49-W-29803 & 4/4/2012 & $51-55$ & $\mathrm{~N}$ & 6.0 & ND & ND \\
\hline SB49 & EVSB49-W-29829 & $5 / 9 / 2013$ & $51-55$ & $\mathrm{~N}$ & 5.6 & ND & ND \\
\hline SB60 & EVSB60-W-23453 & $4 / 2 / 2008$ & $56.7-61.7$ & $\mathrm{~N}$ & 67 & 2.1 & ND \\
\hline SB60 & EVSB60-W-26333 & $4 / 26 / 2009$ & $56.7-61.7$ & $\mathrm{~N}$ & 112 & 3.7 & ND \\
\hline SB60 & EVSB60-W-29450 & $4 / 8 / 2010$ & $56.7-61.7$ & $\mathrm{~N}$ & 86 & 3.2 & ND \\
\hline SB60 & EVSB60-W-29777 & $4 / 28 / 2011$ & $56.7-61.7$ & $\mathrm{~N}$ & 38 & 1.9 & ND \\
\hline SB60 & EVSB60-W-29804 & 4/4/2012 & $56.7-61.7$ & $\mathrm{~N}$ & 38 & 2.0 & ND \\
\hline SB60 & EVSB60-W-29830 & $5 / 9 / 2013$ & $56.7-61.7$ & $\mathrm{~N}$ & 85 & 3.1 & ND \\
\hline SB60 & EVSB60DUP-W-29838 & $5 / 9 / 2013$ & $56.7-61.7$ & DUP-F & 94 & 3.4 & ND \\
\hline SB62 & EVSB62-W-20088 & $3 / 21 / 2006$ & $33-41$ & $\mathrm{~N}$ & $0.7 \mathrm{~J}$ & ND & ND \\
\hline SB62 & EVSB62-W-23454 & $4 / 2 / 2008$ & $33-41$ & $\mathrm{~N}$ & $0.3 \mathrm{~J}$ & ND & ND \\
\hline SB62 & EVSB62-W-26334 & $4 / 25 / 2009$ & $33-41$ & $\mathrm{~N}$ & 1.7 & ND & ND \\
\hline SB62 & EVSB62-W-29751 & $4 / 9 / 2010$ & $33-41$ & $\mathrm{~N}$ & 4.0 & ND & ND \\
\hline SB62 & EVSB62-W-29778 & 4/28/2011 & $33-41$ & $\mathrm{~N}$ & $0.7 \mathrm{~J}$ & ND & ND \\
\hline
\end{tabular}


TABLE 3.2 (Cont.)

\begin{tabular}{|c|c|c|c|c|c|c|c|}
\hline \multirow[b]{2}{*}{ Location } & \multirow[b]{2}{*}{ Sample } & \multirow[b]{2}{*}{$\begin{array}{l}\text { Sample } \\
\text { Date }\end{array}$} & \multirow[b]{2}{*}{$\begin{array}{l}\text { Depth } \\
\text { (ft BGL) }\end{array}$} & \multicolumn{4}{|c|}{ Concentration $(\mu \mathrm{g} / \mathrm{L})$} \\
\hline & & & & $\begin{array}{l}\text { Sample } \\
\text { Type }^{\mathrm{b}}\end{array}$ & $\begin{array}{c}\text { Carbon } \\
\text { Tetrachloride }\end{array}$ & Chloroform & $\begin{array}{l}\text { Methylene } \\
\text { Chloride }\end{array}$ \\
\hline SB62 & EVSB62-W-29805 & $4 / 3 / 2012$ & $33-41$ & $\mathrm{~N}$ & 1.6 & ND & ND \\
\hline SB62 & EVSB62-W-29805DUP & $4 / 3 / 2012$ & $33-41$ & DUP-L & 1.3 & ND & ND \\
\hline SB62 & EVSB62-W-29831 & $5 / 8 / 2013$ & $33-41$ & $\mathrm{~N}$ & ND & ND & ND \\
\hline SB63 & EVSB63-W-20087 & $3 / 21 / 2006$ & $20-25$ & $\mathrm{~N}$ & ND & ND & ND \\
\hline SB63 & EVSB63-W-23455 & $4 / 2 / 2008$ & $20-25$ & $\mathrm{~N}$ & ND & ND & ND \\
\hline SB63 & EVSB63-W-26335 & $4 / 25 / 2009$ & $20-25$ & $\mathrm{~N}$ & ND & ND & ND \\
\hline SB63 & EVSB63-W-29752 & $4 / 9 / 2010$ & $20-25$ & $\mathrm{~N}$ & $0.4 \mathrm{~J}$ & ND & ND \\
\hline SB63 & EVSB63-W-29779 & $4 / 28 / 2011$ & $20-25$ & $\mathrm{~N}$ & ND & ND & ND \\
\hline SB63 & EVSB63-W-29806 & $4 / 4 / 2012$ & $20-25$ & $\mathrm{~N}$ & ND & ND & ND \\
\hline SB63 & EVSB63-W-29832 & $5 / 8 / 2013$ & $20-25$ & $\mathrm{~N}$ & ND & ND & ND \\
\hline SB64 & EVSB64-W-20086 & 3/21/2006 & $22-27$ & $\mathrm{~N}$ & ND & ND & ND \\
\hline SB64 & EVSB64-W-23456 & $4 / 2 / 2008$ & $22-27$ & $\mathrm{~N}$ & ND & ND & ND \\
\hline SB64 & EVSB64-W-26336 & $4 / 25 / 2009$ & $22-27$ & $\mathrm{~N}$ & ND & ND & ND \\
\hline SB64 & EVSB64-W-29753 & $4 / 9 / 2010$ & $22-27$ & $\mathrm{~N}$ & 2.3 & ND & ND \\
\hline SB64 & EVSB64-W-29780 & $4 / 28 / 2011$ & $22-27$ & $\mathrm{~N}$ & ND & ND & ND \\
\hline SB64 & EVSB64-W-29780DUP $^{\mathrm{e}}$ & $4 / 28 / 2011$ & $22-27$ & DUP-L & ND & ND & ND \\
\hline SB64 & EVSB64-W-29807 & $4 / 3 / 2012$ & $22-27$ & $\mathrm{~N}$ & ND & ND & ND \\
\hline SB64 & EVSB64-W-29833 & $5 / 8 / 2013$ & $22-27$ & $\mathrm{~N}$ & ND & ND & ND \\
\hline SB72 & EVSB72-W-20100 & 3/23/2006 & $32-42$ & $\mathrm{~N}$ & $0.4 \mathrm{~J}$ & ND & ND \\
\hline SB72 & EVSB72-W-23458 & $4 / 9 / 2008$ & $32-42$ & $\mathrm{~N}$ & ND & ND & ND \\
\hline SB72 & EVSB72-W-26337 & $4 / 25 / 2009$ & $32-42$ & $\mathrm{~N}$ & ND & ND & ND \\
\hline SB72 & EVSB72-W-29754 & $4 / 8 / 2010$ & $32-42$ & $\mathrm{~N}$ & ND & ND & ND \\
\hline SB72 & EVSB72DUP-W-29759 & $4 / 8 / 2010$ & $32-42$ & DUP-F & ND & ND & ND \\
\hline SB77 & EVSB77-W-20090 & 3/21/2006 & $40-55$ & $\mathrm{~N}$ & ND & ND & ND \\
\hline SB77 & EVSB77-W-23459 & $4 / 1 / 2008$ & $40-55$ & $\mathrm{~N}$ & ND & ND & ND \\
\hline SB77 & EVSB77-W-26338 & $4 / 26 / 2009$ & $40-55$ & $\mathrm{~N}$ & ND & ND & ND \\
\hline SB77 & EVSB77-W-29455 & $4 / 8 / 2010$ & $40-55$ & $\mathrm{~N}$ & ND & ND & ND \\
\hline SB78 & EVSB78-W-20107 & $3 / 24 / 2006$ & $30-40$ & $\mathrm{~N}$ & ND & ND & ND \\
\hline SB78 & EVSB78-W-23460 & $4 / 1 / 2008$ & $30-40$ & $\mathrm{~N}$ & ND & ND & ND \\
\hline SB78 & EVSB78-W-26339 & $4 / 26 / 2009$ & $30-40$ & $\mathrm{~N}$ & ND & ND & ND \\
\hline SB78 & EVSB78-W-29756 & $4 / 8 / 2010$ & $30-40$ & $\mathrm{~N}$ & ND & ND & ND \\
\hline SB78 & EVSB78-W-29781 & $4 / 28 / 2011$ & $30-40$ & $\mathrm{~N}$ & ND & ND & ND \\
\hline SB78 & EVSB78-W-29808 & $4 / 4 / 2012$ & $30-40$ & $\mathrm{~N}$ & ND & ND & ND \\
\hline SB78 & EVSB78-W-29834 & $5 / 9 / 2013$ & $30-40$ & $\mathrm{~N}$ & ND & ND & ND \\
\hline
\end{tabular}


TABLE 3.2 (Cont.)

\begin{tabular}{|c|c|c|c|c|c|c|c|}
\hline \multirow[b]{2}{*}{ Location } & \multirow[b]{2}{*}{ Sample } & \multirow[b]{2}{*}{$\begin{array}{l}\text { Sample } \\
\text { Date }\end{array}$} & \multirow[b]{2}{*}{$\begin{array}{l}\text { Depth } \\
\text { (ft BGL) }\end{array}$} & \multicolumn{4}{|c|}{ Concentration $(\mu \mathrm{g} / \mathrm{L})$} \\
\hline & & & & $\begin{array}{l}\text { Sample } \\
\text { Type }^{\mathrm{b}}\end{array}$ & $\begin{array}{c}\text { Carbon } \\
\text { Tetrachloride }\end{array}$ & Chloroform & $\begin{array}{c}\text { Methylene } \\
\text { Chloride }\end{array}$ \\
\hline SB80 & EVSB80-W-20127 & $3 / 24 / 2006$ & $46.2-70.7$ & $\mathrm{~N}$ & $0.3 \mathrm{~J}$ & ND & ND \\
\hline SB80 & EVSB80-W-23463 & $4 / 2 / 2008$ & $46.2-70.7$ & $\mathrm{~N}$ & $0.8 \mathrm{~J}$ & ND & ND \\
\hline SB80 & EVSB80-W-26340 & $4 / 25 / 2009$ & $46.2-70.7$ & $\mathrm{~N}$ & 1.3 & ND & ND \\
\hline SB80 & EVSB80-W-29757 & 4/8/2010 & $46.2-70.7$ & $\mathrm{~N}$ & 1.7 & ND & ND \\
\hline SB80 & EVSB80-W-29782 & $4 / 27 / 2011$ & $46.2-70.7$ & $\mathrm{~N}$ & 1.4 & ND & ND \\
\hline SB80 & EVSB80-W-29809 & 4/4/2012 & $46.2-70.7$ & $\mathrm{~N}$ & 2.2 & ND & ND \\
\hline SB80 & EVSB80-W-29835 & $5 / 9 / 2013$ & $46.2-70.7$ & $\mathrm{~N}$ & 2.1 & ND & ND \\
\hline SB88 & EVSB88-W-20124 & $3 / 24 / 2006$ & $62-72$ & $\mathrm{~N}$ & 114 & 3.2 & ND \\
\hline SB88 & EVSB88-W-23464 & $4 / 8 / 2008$ & $62-72$ & $\mathrm{~N}$ & 159 & 5.0 & ND \\
\hline SB88 & EVSB88-W-26341 & $4 / 26 / 2009$ & $62-72$ & $\mathrm{~N}$ & 234 & 7.9 & ND \\
\hline SB88 & EVSB88-W-29758 & 4/8/2010 & $62-72$ & $\mathrm{~N}$ & 212 & 7.6 & ND \\
\hline SB88 & EVSB88-W-29783 & $4 / 28 / 2011$ & $62-72$ & $\mathrm{~N}$ & 69 & 3.8 & ND \\
\hline SB88 & EVSB88-W-29810 & 4/4/2012 & $62-72$ & $\mathrm{~N}$ & 107 & 5.3 & ND \\
\hline SB88 & EVSB88-W-29810DUP & $4 / 4 / 2012$ & $62-72$ & DUP-L & 105 & 5.1 & ND \\
\hline SB88 & EVSB88DUP-W-29812 & $4 / 4 / 2012$ & $62-72$ & DUP-F & 119 & 5.2 & ND \\
\hline SB88 & EVSB88-W-29836 & $5 / 9 / 2013$ & $62-72$ & $\mathrm{~N}$ & 101 & 4.4 & ND \\
\hline
\end{tabular}

a Sampling at SB72 and SB77 was discontinued with KDHE (2010) approval.

b Sample types: DUP-F, field replicate; DUP-L, duplicate laboratory analysis; N, primary sample.

c ND, compound analyzed for but not detected at a level greater than or equal to the method detection limit $(<1 \mu \mathrm{g} / \mathrm{L})$.

d J, compound identified with an estimated concentration between the instrument detection limit and the method detection limit.

e Quality control sample. 
TABLE 3.3 Field measurements for groundwater samples collected in 2001-2013 at monitoring locations in the approved monitoring network. ${ }^{a}$

\begin{tabular}{|c|c|c|c|c|c|c|c|c|c|c|}
\hline Location & Sample & $\begin{array}{l}\text { Sample } \\
\text { Date }\end{array}$ & $\begin{array}{l}\text { Depth } \\
\text { (ft BGL) }\end{array}$ & $\begin{array}{c}\text { Temperature } \\
\left({ }^{\circ} \mathrm{C}\right)\end{array}$ & $\mathrm{pH}$ & $\begin{array}{l}\text { Conductivity } \\
(\mu \mathrm{S} / \mathrm{cm})\end{array}$ & $\begin{array}{c}\text { Dissolved } \\
\text { Oxygen } \\
\text { (mg/L) }\end{array}$ & $\begin{array}{c}\text { Oxidation- } \\
\text { Reduction } \\
\text { Potential } \\
(\mathrm{mV})\end{array}$ & $\begin{array}{c}\text { Carbon } \\
\text { Dioxide } \\
(\mathrm{mg} / \mathrm{L})\end{array}$ & $\begin{array}{c}\text { Iron (II) } \\
\text { (mg/L) }\end{array}$ \\
\hline MW1 & EVMW1-W-20103 & $3 / 23 / 2006$ & $41-51$ & 13.1 & 7.26 & 1236 & - & - & - & - \\
\hline MW1 & EVMW1-W-23441 & $4 / 1 / 2008$ & $41-51$ & 9.9 & 6.89 & 1251 & 2.06 & 201 & - & 0.00 \\
\hline MW1 & EVMW1-W-26326 & $4 / 25 / 2009$ & $41-51$ & 13.0 & 6.85 & 1039 & 1.87 & 167 & - & - \\
\hline MW1 & EVMW1-W-29743 & $4 / 8 / 2010$ & $41-51$ & 11.9 & 6.78 & 945 & 1.87 & 96 & - & - \\
\hline MW1 & EVMW1-W-29770 & $4 / 27 / 2011$ & $41-51$ & 12.6 & 6.83 & 898 & 2.15 & 101 & - & 0.00 \\
\hline MW1 & EVMW1-W-29797 & $4 / 3 / 2012$ & $41-51$ & 13.0 & 6.97 & 878 & 0.66 & -65 & - & 0.00 \\
\hline MW1 & EVMW1-W-29823 & $5 / 8 / 2013$ & $41-51$ & 15.4 & 6.98 & 887 & 0.83 & -19 & - & 0.06 \\
\hline MW2 & EVMW2-W-23442 & 4/9/2008 & $59-79$ & 12.7 & 7.51 & 693 & 6.08 & 173 & - & 0.01 \\
\hline MW2 & EVMW2-W-26327 & $4 / 26 / 2009$ & $59-79$ & 17.3 & 7.14 & 585 & 5.46 & 173 & - & - \\
\hline MW2 & EVMW2-W-29744 & $4 / 8 / 2010$ & $59-79$ & 15.2 & 7.12 & 555 & 6.33 & 256 & - & 0.00 \\
\hline MW2 & EVMW2-W-29771 & $4 / 27 / 2011$ & $59-79$ & 13.3 & 7.62 & 528 & 9.83 & 105 & - & 0.04 \\
\hline MW2 & EVMW2-W-29798 & 4/4/2012 & $59-79$ & 14.0 & 6.74 & 537 & 5.81 & 334 & - & 0.04 \\
\hline MW2 & EVMW2-W-29824 & $5 / 9 / 2013$ & $59-79$ & 13.8 & 7.36 & 513 & 5.12 & 250 & - & 0.04 \\
\hline MW3 & EVMW3-W-23443 & 4/1/2008 & $56.5-71.5$ & 11.0 & 7.18 & 746 & 2.65 & 150 & - & 0.18 \\
\hline MW3 & EVMW3-W-26328 & $4 / 25 / 2009$ & $56.5-71.5$ & 13.5 & 7.36 & 660 & 2.88 & -82 & - & - \\
\hline MW3 & EVMW3-W-29745 & $4 / 8 / 2010$ & $56.5-71.5$ & 13.3 & 6.91 & 564 & 2.93 & -2 & - & 0.04 \\
\hline MW3 & EVMW3-W-29772 & $4 / 28 / 2011$ & $56.5-71.5$ & 13.8 & 7.37 & 534 & 3.50 & 91 & - & 0.03 \\
\hline MW3 & EVMW3-W-29799 & 4/4/2012 & $56.5-71.5$ & 13.2 & 7.38 & 550 & 3.89 & -18 & - & 0.07 \\
\hline MW3 & EVMW3-W-29825 & $5 / 8 / 2013$ & $56.5-71.5$ & 14.6 & 7.35 & 529 & 3.47 & 15 & - & 0.09 \\
\hline MW4 & EVMW4-W-20117 & $3 / 23 / 2006$ & $48.5-68.5$ & 12.8 & 7.17 & 675 & 0.40 & 218 & 25 & 0.21 \\
\hline MW4 & EVMW4-W-23444 & $4 / 2 / 2008$ & $48.5-68.5$ & 9.7 & 7.25 & 817 & 3.03 & 185 & - & 0.00 \\
\hline MW4 & EVMW4-W-29746 & $4 / 9 / 2010$ & $48.5-68.5$ & 13.1 & 7.13 & 628 & 2.81 & 178 & - & 0.00 \\
\hline MW4 & EVMW4-W-29773 & $4 / 28 / 2011$ & $48.5-68.5$ & 15.4 & 7.27 & 612 & 3.11 & 99 & - & 0.02 \\
\hline MW4 & EVMW4-W-29800 & $4 / 3 / 2012$ & $48.5-68.5$ & 13.4 & 7.33 & 605 & 3.58 & 26 & - & 0.02 \\
\hline MW4 & EVMW4-W-29826 & $5 / 8 / 2013$ & $48.5-68.5$ & 15.3 & 7.40 & 591 & 3.74 & 20 & - & 0.00 \\
\hline
\end{tabular}


TABLE 3.3 (Cont.)

\begin{tabular}{|c|c|c|c|c|c|c|c|c|c|c|}
\hline Location & Sample & $\begin{array}{l}\text { Sample } \\
\text { Date }\end{array}$ & $\begin{array}{l}\text { Depth } \\
\text { (ft BGL) }\end{array}$ & $\begin{array}{c}\text { Temperature } \\
\left({ }^{\circ} \mathrm{C}\right)\end{array}$ & $\mathrm{pH}$ & $\begin{array}{l}\text { Conductivity } \\
(\mu S / \mathrm{cm})\end{array}$ & $\begin{array}{l}\text { Dissolved } \\
\text { Oxygen } \\
(\mathrm{mg} / \mathrm{L})\end{array}$ & $\begin{array}{l}\text { Oxidation- } \\
\text { Reduction } \\
\text { Potential } \\
(\mathrm{mV})\end{array}$ & $\begin{array}{c}\text { Carbon } \\
\text { Dioxide } \\
(\mathrm{mg} / \mathrm{L})\end{array}$ & $\begin{array}{c}\text { Iron (II) } \\
\text { (mg/L) }\end{array}$ \\
\hline SB09 & EVSB09-W-20098 & $3 / 22 / 2006$ & $51-57$ & 10.2 & 7.42 & 862 & - & - & - & - \\
\hline SB09 & EVSB09-W-23448 & 4/9/2008 & $51-57$ & 9.0 & 7.36 & 616 & 2.71 & 186 & - & 0.00 \\
\hline SB09 & EVSB09-W-26330 & $4 / 26 / 2009$ & $51-57$ & 13.5 & 7.05 & 692 & 2.22 & 210 & - & - \\
\hline SB09 & EVSB09-W-29747 & 4/9/2010 & $51-57$ & 19.0 & 6.97 & 612 & 3.91 & 223 & - & 0.01 \\
\hline SB09 & EVSB09-W-29774 & $4 / 27 / 2011$ & $51-57$ & 13.8 & 7.05 & 645 & 6.31 & 106 & - & 0.15 \\
\hline SB09 & EVSB09-W-29801 & 4/4/2012 & $51-57$ & 13.4 & 6.64 & 666 & 5.14 & 357 & - & 0.11 \\
\hline SB09 & EVSB09-W-29827 & $5 / 9 / 2013$ & $51-57$ & 15.6 & 7.34 & 665 & 2.27 & -9 & - & 0.06 \\
\hline SB34 & EVSB34-W-12857 & $3 / 28 / 2001$ & $46-49$ & 14.7 & 7.87 & 767 & - & - & - & - \\
\hline SB34 & EVSB34-W-12854 & $3 / 28 / 2001$ & $49-53$ & 13.2 & 7.57 & 757 & - & - & - & - \\
\hline SB34 & EVSB34-W-20097 & $3 / 22 / 2006$ & $46-53$ & 11.3 & 7.31 & 781 & - & - & - & - \\
\hline SB34 & EVSB34-W-23451 & $4 / 9 / 2008$ & $46-53$ & 10.5 & 7.08 & 924 & 0.98 & 178 & - & 0.05 \\
\hline SB34 & EVSB34-W-26331 & $4 / 26 / 2009$ & $46-53$ & 13.6 & 7.18 & 858 & 1.62 & -146 & - & - \\
\hline SB34 & EVSB34-W-29748 & 4/9/2010 & $46-53$ & 13.9 & 6.79 & 769 & 1.10 & 131 & - & 0.00 \\
\hline SB34 & EVSB34-W-29775 & $4 / 27 / 2011$ & $46-53$ & 12.8 & 6.84 & 716 & 1.36 & 70 & - & 0.02 \\
\hline SB34 & EVSB34-W-29802 & $4 / 4 / 2012$ & $46-53$ & 13.0 & 7.05 & 715 & 0.90 & -103 & - & 0.00 \\
\hline SB34 & EVSB34-W-29828 & $5 / 8 / 2013$ & $46-53$ & 16.3 & 7.13 & 660 & 2.38 & 8 & - & 0.03 \\
\hline SB49 & EVSB49-W-20095 & $3 / 22 / 2006$ & $51-55$ & 11.3 & 7.15 & 649 & - & - & - & - \\
\hline SB49 & EVSB49-W-23452 & $4 / 8 / 2008$ & $51-55$ & 9.4 & 7.68 & 618 & 3.79 & 136 & - & 0.04 \\
\hline SB49 & EVSB49-W-26332 & $4 / 26 / 2009$ & $51-55$ & 15.5 & 7.54 & 583 & 6.70 & -140 & - & - \\
\hline SB49 & EVSB49-W-29749 & $4 / 8 / 2010$ & $51-55$ & 14.1 & 7.14 & 505 & 5.02 & 58 & - & 0.12 \\
\hline SB49 & EVSB49-W-29776 & $4 / 28 / 2011$ & $51-55$ & 15.5 & 7.36 & 497 & 5.16 & 138 & - & 0.00 \\
\hline SB49 & EVSB49-W-29803 & $4 / 4 / 2012$ & $51-55$ & 14.2 & 7.45 & 504 & 5.22 & -9 & - & 0.01 \\
\hline SB49 & EVSB49-W-29829 & $5 / 9 / 2013$ & $51-55$ & 13.4 & 7.36 & 469 & 5.64 & 22 & - & 0.04 \\
\hline SB60 & EVSB60-W-23453 & $4 / 2 / 2008$ & $56.7-61.7$ & 14.7 & 7.58 & 660 & 4.10 & 211 & - & 0.00 \\
\hline SB60 & EVSB60-W-26333 & $4 / 26 / 2009$ & $56.7-61.7$ & 17.4 & 7.16 & 630 & 5.26 & 179 & - & - \\
\hline SB60 & EVSB60-W-29450 & 4/8/2010 & $56.7-61.7$ & 17.8 & 7.18 & 623 & 5.80 & 240 & - & 0.00 \\
\hline SB60 & EVSB60-W-29777 & $4 / 28 / 2011$ & $56.7-61.7$ & 18.9 & 7.20 & 643 & 11.12 & 141 & - & 0.18 \\
\hline SB60 & EVSB60-W-29804 & 4/4/2012 & $56.7-61.7$ & 15.4 & 6.64 & 392 & 12.35 & 339 & - & - \\
\hline SB60 & EVSB60-W-29830 & $5 / 9 / 2013$ & $56.7-61.7$ & 14.5 & 7.42 & 587 & 5.88 & 242 & - & 0.06 \\
\hline SB62 & EVSB62-W-20088 & $3 / 21 / 2006$ & $33-41$ & 11.5 & 7.52 & 722 & - & - & - & - \\
\hline SB62 & EVSB62-W-23454 & $4 / 2 / 2008$ & $33-41$ & 7.0 & 7.75 & 761 & 4.33 & 221 & - & 0.00 \\
\hline SB62 & EVSB62-W-26334 & $4 / 25 / 2009$ & $33-41$ & 12.0 & 7.24 & 696 & 6.17 & 156 & - & - \\
\hline
\end{tabular}


TABLE 3.3 (Cont.)

\begin{tabular}{|c|c|c|c|c|c|c|c|c|c|c|}
\hline Location & Sample & $\begin{array}{l}\text { Sample } \\
\text { Date }\end{array}$ & $\begin{array}{c}\text { Depth } \\
\text { (ft BGL) }\end{array}$ & $\begin{array}{c}\text { Temperature } \\
\left({ }^{\circ} \mathrm{C}\right)\end{array}$ & $\mathrm{pH}$ & $\begin{array}{l}\text { Conductivity } \\
(\mu S / \mathrm{cm})\end{array}$ & $\begin{array}{c}\text { Dissolved } \\
\text { Oxygen } \\
(\mathrm{mg} / \mathrm{L})\end{array}$ & $\begin{array}{l}\text { Oxidation- } \\
\text { Reduction } \\
\text { Potential } \\
(\mathrm{mV})\end{array}$ & $\begin{array}{c}\text { Carbon } \\
\text { Dioxide } \\
(\mathrm{mg} / \mathrm{L})\end{array}$ & $\begin{array}{c}\text { Iron (II) } \\
\text { (mg/L) }\end{array}$ \\
\hline SB62 & EVSB62-W-29751 & 4/9/2010 & $33-41$ & 20.4 & 7.26 & 628 & 6.42 & 238 & - & 0.00 \\
\hline SB62 & EVSB62-W-29778 & $4 / 28 / 2011$ & $33-41$ & 22.1 & 7.37 & 697 & 9.81 & 115 & - & 0.12 \\
\hline SB62 & EVSB62-W-29805 & 4/3/2012 & $33-41$ & 15.2 & 6.84 & 598 & 6.82 & 367 & - & 0.01 \\
\hline SB62 & EVSB62-W-29831 & $5 / 8 / 2013$ & $33-41$ & 17.0 & 7.36 & 595 & 6.04 & 203 & - & 0.01 \\
\hline SB63 & EVSB63-W-20087 & $3 / 21 / 2006$ & $20-25$ & 6.5 & 6.92 & 688 & - & - & - & - \\
\hline SB63 & EVSB63-W-23455 & $4 / 2 / 2008$ & $20-25$ & 14.7 & 6.62 & 707 & 0.74 & 158 & - & 0.08 \\
\hline SB63 & EVSB63-W-26335 & $4 / 25 / 2009$ & $20-25$ & 12.0 & 6.46 & 606 & 1.01 & -63 & - & - \\
\hline SB63 & EVSB63-W-29752 & 4/9/2010 & $20-25$ & 18.4 & 6.28 & 505 & 0.35 & 68 & - & 0.00 \\
\hline SB63 & EVSB63-W-29779 & $4 / 28 / 2011$ & $20-25$ & 20.2 & 6.21 & 630 & 1.92 & 69 & - & 0.41 \\
\hline SB63 & EVSB63-W-29806 & 4/4/2012 & $20-25$ & 13.6 & 6.10 & 548 & 0.70 & -4 & - & 0.42 \\
\hline SB63 & EVSB63-W-29832 & $5 / 8 / 2013$ & $20-25$ & 15.2 & 6.78 & 560 & 0.50 & 76 & - & 0.66 \\
\hline SB64 & EVSB64-W-20086 & $3 / 21 / 2006$ & $22-27$ & 11.3 & 7.27 & 876 & - & - & - & - \\
\hline SB64 & EVSB64-W-23456 & $4 / 2 / 2008$ & $22-27$ & 10.5 & 7.07 & 826 & 1.21 & 169 & - & 0.16 \\
\hline SB64 & EVSB64-W-26336 & $4 / 25 / 2009$ & $22-27$ & 11.2 & 6.79 & 784 & 2.45 & -46 & - & - \\
\hline SB64 & EVSB64-W-29753 & 4/9/2010 & $22-27$ & 15.5 & 6.67 & 708 & 1.45 & 256 & - & 0.00 \\
\hline SB64 & EVSB64-W-29780 & $4 / 28 / 2011$ & $22-27$ & 17.6 & 6.47 & 749 & 1.09 & 116 & - & 0.04 \\
\hline SB64 & EVSB64-W-29807 & 4/3/2012 & $22-27$ & 14.3 & 6.25 & 678 & 1.37 & 368 & - & 0.00 \\
\hline SB64 & EVSB64-W-29833 & $5 / 8 / 2013$ & $22-27$ & 15.9 & 6.86 & 659 & 3.29 & 200 & - & 0.03 \\
\hline SB72 & EVSB72-W-20100 & $3 / 23 / 2006$ & $32-42$ & 12.9 & 7.28 & 586 & - & - & - & - \\
\hline SB72 & EVSB72-W-23458 & $4 / 9 / 2008$ & $32-42$ & 12.9 & 7.08 & 593 & 4.73 & 182 & - & 0.10 \\
\hline SB72 & EVSB72-W-26337 & $4 / 25 / 2009$ & $32-42$ & 12.7 & 7.01 & 553 & 5.89 & 163 & - & - \\
\hline SB72 & EVSB72-W-29754 & 4/8/2010 & $32-42$ & 16.3 & 7.03 & 527 & 5.43 & 80 & - & 0.00 \\
\hline SB77 & EVSB77-W-20090 & $3 / 21 / 2006$ & $40-55$ & 11.9 & 7.58 & 692 & - & - & - & - \\
\hline SB77 & EVSB77-W-23459 & $4 / 1 / 2008$ & $40-55$ & 9.9 & 7.60 & 653 & 5.44 & 163 & - & 0.12 \\
\hline SB77 & EVSB77-W-26338 & $4 / 26 / 2009$ & $40-55$ & 19.9 & 7.45 & 709 & 8.88 & -122 & - & - \\
\hline SB77 & EVSB77-W-29455 & 4/8/2010 & $40-55$ & 15.9 & 7.09 & 671 & 6.89 & 98 & - & 0.08 \\
\hline SB78 & EVSB78-W-20107 & $3 / 24 / 2006$ & $30-40$ & 12.2 & 7.41 & 653 & - & - & - & - \\
\hline SB78 & EVSB78-W-23460 & 4/1/2008 & $30-40$ & 10.1 & 7.64 & 621 & 4.74 & 161 & - & 0.00 \\
\hline SB78 & EVSB78-W-26339 & $4 / 26 / 2009$ & $30-40$ & 18.2 & 7.54 & 597 & 6.73 & -134 & - & - \\
\hline SB78 & EVSB78-W-29756 & $4 / 8 / 2010$ & $30-40$ & 16.3 & 7.18 & 513 & 5.06 & 89 & - & 0.09 \\
\hline SB78 & EVSB78-W-29781 & $4 / 28 / 2011$ & $30-40$ & 19.1 & 7.38 & 543 & 5.48 & 150 & - & 0.00 \\
\hline
\end{tabular}


TABLE 3.3 (Cont.)

\begin{tabular}{|c|c|c|c|c|c|c|c|c|c|c|}
\hline Location & Sample & $\begin{array}{l}\text { Sample } \\
\text { Date }\end{array}$ & $\begin{array}{l}\text { Depth } \\
\text { (ft BGL) }\end{array}$ & $\begin{array}{c}\text { Temperature } \\
\left({ }^{\circ} \mathrm{C}\right)\end{array}$ & $\mathrm{pH}$ & $\begin{array}{l}\text { Conductivity } \\
(\mu \mathrm{S} / \mathrm{cm})\end{array}$ & $\begin{array}{c}\text { Dissolved } \\
\text { Oxygen } \\
(\mathrm{mg} / \mathrm{L})\end{array}$ & $\begin{array}{l}\text { Oxidation- } \\
\text { Reduction } \\
\text { Potential } \\
(\mathrm{mV})\end{array}$ & $\begin{array}{c}\text { Carbon } \\
\text { Dioxide } \\
(\mathrm{mg} / \mathrm{L})\end{array}$ & $\begin{array}{c}\text { Iron (II) } \\
\text { (mg/L) }\end{array}$ \\
\hline SB78 & EVSB78-W-29808 & $4 / 4 / 2012$ & $30-40$ & 16.1 & 7.43 & 521 & 5.80 & 4 & - & 0.05 \\
\hline SB78 & EVSB78-W-29834 & $5 / 9 / 2013$ & $30-40$ & 13.9 & 7.48 & 468 & 6.26 & 19 & - & 0.12 \\
\hline SB80 & EVSB80-W-20127 & $3 / 24 / 2006$ & $46.2-70.7$ & 12.3 & 7.14 & 679 & 3.30 & 212 & 25 & 0.46 \\
\hline SB80 & EVSB80-W-23463 & $4 / 2 / 2008$ & $46.2-70.7$ & 17.2 & 7.14 & 706 & 5.30 & 222 & - & 0.00 \\
\hline SB80 & EVSB80-W-26340 & $4 / 25 / 2009$ & $46.2-70.7$ & 15.3 & 7.21 & 592 & 4.78 & 164 & - & - \\
\hline SB80 & EVSB80-W-29757 & $4 / 8 / 2010$ & $46.2-70.7$ & 16.6 & 7.19 & 574 & 5.52 & 268 & - & 0.04 \\
\hline SB80 & EVSB80-W-29782 & $4 / 27 / 2011$ & $46.2-70.7$ & 18.1 & 7.19 & 621 & 4.92 & 128 & - & 0.24 \\
\hline SB80 & EVSB80-W-29809 & $4 / 4 / 2012$ & $46.2-70.7$ & 14.6 & 7.39 & 566 & 4.61 & -6 & - & 0.11 \\
\hline SB80 & EVSB80-W-29835 & $5 / 9 / 2013$ & $46.2-70.7$ & 13.9 & 7.37 & 531 & 4.01 & 254 & - & 0.18 \\
\hline SB88 & EVSB88-W-20124 & $3 / 24 / 2006$ & $62-72$ & 12.9 & 6.96 & 717 & 4.58 & 183 & 25 & 0.46 \\
\hline SB88 & EVSB88-W-23464 & $4 / 8 / 2008$ & $62-72$ & 8.2 & 7.23 & 758 & 5.23 & 242 & - & 0.11 \\
\hline SB88 & EVSB88-W-26341 & $4 / 26 / 2009$ & $62-72$ & 18.0 & 7.16 & 628 & 4.19 & 182 & - & - \\
\hline SB88 & EVSB88-W-29758 & $4 / 8 / 2010$ & $62-72$ & 15.9 & 7.12 & 592 & 4.83 & 227 & - & 0.00 \\
\hline SB88 & EVSB88-W-29783 & $4 / 28 / 2011$ & $62-72$ & 20.1 & 7.30 & 627 & 9.65 & 143 & - & 0.16 \\
\hline SB88 & EVSB88-W-29810 & 4/4/2012 & $62-72$ & 15.5 & 6.81 & 577 & 6.52 & 346 & - & 0.10 \\
\hline SB88 & EVSB88-W-29836 & $5 / 9 / 2013$ & $62-72$ & 17.7 & 7.42 & 573 & 4.45 & 229 & - & 0.04 \\
\hline
\end{tabular}

a Sampling at SB72 and SB77 was discontinued with KDHE (2010) approval. 
TABLE 3.4 Analytical results for VOCs in surface water samples collected during annual monitoring in 2009-2013.

\begin{tabular}{|c|c|c|c|c|}
\hline \multirow[b]{2}{*}{ Location } & \multirow[b]{2}{*}{ Sample } & \multirow[b]{2}{*}{$\begin{array}{l}\text { Sample } \\
\text { Date }\end{array}$} & \multicolumn{2}{|c|}{ Concentration $(\mu \mathrm{g} / \mathrm{L})$} \\
\hline & & & $\begin{array}{c}\text { Carbon } \\
\text { Tetrachloride }\end{array}$ & Chloroform \\
\hline EV001 & EV001-W-26320 & $4 / 24 / 2009$ & $N D^{a}$ & ND \\
\hline EV003 & EV003-W-26321 & $4 / 24 / 2009$ & ND & ND \\
\hline EV005 & EV005-W-26322 & $4 / 24 / 2009$ & ND & ND \\
\hline EV006 & EV006-W-26323 & $4 / 24 / 2009$ & ND & ND \\
\hline EV008 & EV008-W-26324 & $4 / 24 / 2009$ & ND & ND \\
\hline EV001 & EV001-W-29738 & 4/8/2010 & ND & ND \\
\hline EV003 & EV003-W-29739 & 4/8/2010 & ND & ND \\
\hline EV005 & EV005-W-29740 & $4 / 8 / 2010$ & ND & ND \\
\hline EV006 & EV006-W-29741 & 4/8/2010 & ND & ND \\
\hline EV008 & EV008-W-29742 & $4 / 8 / 2010$ & ND & ND \\
\hline EV001 & EV001-W-29765 & 4/29/2011 & ND & ND \\
\hline EV003 & EV003-W-29766 & 4/29/2011 & ND & ND \\
\hline EV005 & EV005-W-29767 & $4 / 29 / 2011$ & ND & ND \\
\hline EV006 & EV006-W-29768 & $4 / 29 / 2011$ & ND & ND \\
\hline EV008 & EV008-W-29769 & 4/29/2011 & ND & ND \\
\hline EV001 & EV001-W-29792 & 4/3/2012 & ND & ND \\
\hline EV003 & EV003-W-29793 & 4/3/2012 & ND & ND \\
\hline EV005 & EV005-W-29794 & 4/3/2012 & ND & ND \\
\hline EV006 & EV006-W-29795 & $4 / 3 / 2012$ & ND & ND \\
\hline EV008 & EV008-W-29796 & $4 / 3 / 2012$ & ND & ND \\
\hline EV001 & EV001-W-29818 & $5 / 8 / 2013$ & ND & ND \\
\hline EV003 & EV003-W-29819 & $5 / 8 / 2013$ & ND & ND \\
\hline EV005 & EV005-W-29820 & $5 / 8 / 2013$ & ND & ND \\
\hline EV006 & EV006-W-29821 & $5 / 8 / 2013$ & ND & ND \\
\hline EV008 & EV008-W-29822 & $5 / 8 / 2013$ & ND & ND \\
\hline
\end{tabular}

a ND, Compound analyzed for but not detected at a level greater than or equal to the method detection limit $(<1 \mu \mathrm{g} / \mathrm{L})$. 
TABLE 3.5 Analytical results for VOCs in tree branch samples collected on September 24, 2013. ${ }^{2}$

\begin{tabular}{|c|c|c|c|c|}
\hline \multirow[b]{2}{*}{ Location } & \multirow[b]{2}{*}{ Sample } & \multirow{2}{*}{$\begin{array}{l}\text { Height } \\
\text { (ft above } \\
\text { ground) }\end{array}$} & \multicolumn{2}{|c|}{ Concentration $(\mu \mathrm{g} / \mathrm{kg})$} \\
\hline & & & $\begin{array}{c}\text { Carbon } \\
\text { Tetrachloride }\end{array}$ & Chloroform \\
\hline EV027 & EV027-B-29792 & 4 & $\mathrm{ND}^{\mathrm{b}}$ & ND \\
\hline EV028 & EV028-B-29793 & 3 & ND & ND \\
\hline EV004 & EV004-B-29795 & 3 & ND & ND \\
\hline EV013A & EV013A-B-29794 & 2 & ND & ND \\
\hline EV016 & EV016-B-29797 & 4 & ND & ND \\
\hline EV021 & EV021-B-29796 & 4 & ND & ND \\
\hline EV022 & EV022-B-29798 & 4 & ND & ND \\
\hline EV024 & EV024-B-29799 & 6 & ND & ND \\
\hline EV017 & EV017-B-29800 & 1 & ND & ND \\
\hline EV018 & EV018-B-29801 & 2 & ND & ND \\
\hline EV006 & EV006-B-29802 & 3 & ND & ND \\
\hline EV019 & EV019-B-29803 & 4 & ND & ND \\
\hline EV020 & EV020-B-29804 & 6 & ND & ND \\
\hline EV008A & EV008A-B-29805 & 3 & ND & ND \\
\hline EV026 & EV026-B-29806 & 6 & ND & ND \\
\hline EV010 & EV010-B-29807 & 3 & ND & ND \\
\hline EV009 & EV009-B-29808 & 4 & ND & ND \\
\hline EV029 & EV029-B-29809 & 8 & ND & ND \\
\hline EV030 & EV030-B-29810 & 5 & ND & ND \\
\hline EV031 & EV031-B-29811 & 1 & ND & ND \\
\hline
\end{tabular}

a Analysis was by EPA Method SW3810.

b ND, compound analyzed for but not detected at a level greater than or equal to the method detection limit $(<0.1 \mu \mathrm{g} / \mathrm{kg}$ for carbon tetrachloride or $<0.75 \mu \mathrm{g} / \mathrm{kg}$ for chloroform). 
TABLE 3.6 Analytical results for tree branch samples collected in 20062013.

\begin{tabular}{|c|c|c|c|c|}
\hline \multirow[b]{2}{*}{ Location } & \multirow[b]{2}{*}{ Sample } & \multirow[b]{2}{*}{$\begin{array}{l}\text { Sample } \\
\text { Date }\end{array}$} & \multicolumn{2}{|c|}{ Concentration $(\mu \mathrm{g} / \mathrm{kg})$} \\
\hline & & & $\begin{array}{c}\text { Carbon } \\
\text { Tetrachloride }\end{array}$ & Chloroform \\
\hline EV001 & EV001-B-18985 & $10 / 14 / 2006$ & $N D^{a}$ & ND \\
\hline EV002 & EV002-B-18987 & $10 / 14 / 2006$ & ND & 1.6 \\
\hline EV003 & EV003-B-18989 & $10 / 14 / 2006$ & ND & ND \\
\hline EV004 & EV004-B-18991 & $10 / 14 / 2006$ & ND & 1.2 \\
\hline EV004 & EV004-B-23360 & $4 / 2 / 2007$ & ND & $\mathrm{ND}$ \\
\hline EV004 & EV004-B-23361 & $4 / 2 / 2007$ & ND & ND \\
\hline EV004 & EV004-B-23378 & $7 / 26 / 2007$ & ND & ND \\
\hline EV004 & EV004-B-20137 & $7 / 25 / 2008$ & ND & ND \\
\hline EV004 & EV004-B-29721 & 8/28/2009 & ND & ND \\
\hline EV004 & EV004-B-31721 & $7 / 28 / 2010$ & ND & ND \\
\hline EV004 & EV004-B-29751 & $7 / 29 / 2011$ & ND & ND \\
\hline EV004 & EV004-B-29773 & $7 / 12 / 2012$ & 0.34 & ND \\
\hline EV004 & EV004-B-29795 & $9 / 24 / 2013$ & ND & ND \\
\hline EV005 & EV005-B-18993 & $10 / 14 / 2006$ & ND & ND \\
\hline EV005 & EV005-B-23365 & $4 / 2 / 2007$ & ND & ND \\
\hline EV005 & EV005-B-23366 & $4 / 2 / 2007$ & ND & ND \\
\hline EV005 & EV005-B-23384 & $7 / 26 / 2007$ & ND & 0.76 \\
\hline EV005 & EV005-B-20142 & $7 / 25 / 2008$ & ND & ND \\
\hline EV005 & EV005-B-29726 & 8/28/2009 & ND & ND \\
\hline EV005 & EV005-B-31725 & $7 / 28 / 2010$ & ND & ND \\
\hline EV005 & EV005-B-29756 & $7 / 29 / 2011$ & ND & ND \\
\hline EV006 & EV006-B-18995 & $10 / 14 / 2006$ & ND & ND \\
\hline EV006 & EV006-B-23369 & $4 / 2 / 2007$ & ND & 1.1 \\
\hline EV006 & EV006-B-23370 & $4 / 2 / 2007$ & ND & 1.2 \\
\hline EV006 & EV006-B-23392 & $7 / 26 / 2007$ & ND & $\mathrm{ND}$ \\
\hline EV006 & EV006-B-20146 & $7 / 25 / 2008$ & ND & ND \\
\hline EV006 & EV006-B-29730 & $8 / 28 / 2009$ & ND & ND \\
\hline EV006 & EV006-B-31729 & $7 / 28 / 2010$ & ND & ND \\
\hline EV006 & EV006-B-29760 & $7 / 29 / 2011$ & ND & ND \\
\hline EV006 & EV006-B-29780 & $7 / 12 / 2012$ & 0.35 & ND \\
\hline EV006 & EV006-B-29802 & $9 / 24 / 2013$ & ND & ND \\
\hline EV007 & EV007-B-18997 & $10 / 14 / 2006$ & ND & 0.83 \\
\hline EV007 & EV007-B-23372 & $4 / 2 / 2007$ & ND & ND \\
\hline EV007 & EV007-B-23400 & $7 / 26 / 2007$ & ND & ND \\
\hline EV007 & Not sampled & $7 / 25 / 2008$ & - & .- \\
\hline EV007 & EV007-B-29732 & $8 / 28 / 2009$ & ND & ND \\
\hline EV007 & EV007-no sample & $7 / 29 / 2011$ & - & - \\
\hline EV008 & EV008-B-18999 & $10 / 14 / 2006$ & ND & ND \\
\hline EV008 & EV008-B-23374 & $4 / 2 / 2007$ & ND & 1.6 \\
\hline EV008 & EV008-B-23404 & $7 / 26 / 2007$ & ND & ND \\
\hline EV008 & EV008-B-20151 & $7 / 25 / 2008$ & ND & ND \\
\hline EV008 & EV008-B-29735 & 8/28/2009 & ND & ND \\
\hline EV008 & EV008-B-31731 & $7 / 28 / 2010$ & ND & ND \\
\hline
\end{tabular}


TABLE 3.6 (Cont.)

\begin{tabular}{|c|c|c|c|c|}
\hline \multirow[b]{2}{*}{ Location } & \multirow[b]{2}{*}{ Sample } & \multirow[b]{2}{*}{$\begin{array}{l}\text { Sample } \\
\text { Date }\end{array}$} & \multicolumn{2}{|c|}{ Concentration $(\mu \mathrm{g} / \mathrm{kg})$} \\
\hline & & & $\begin{array}{c}\text { Carbon } \\
\text { Tetrachloride }\end{array}$ & Chloroform \\
\hline EV008A & EV008A-B-29765 & $7 / 29 / 2011$ & ND & 0.87 \\
\hline EV008A & EV008A-B-29783 & $7 / 12 / 2012$ & Broken & Broken \\
\hline EV008A & EV008A-B-29805 & $9 / 24 / 2013$ & ND & ND \\
\hline EV009 & EV009-B-19001 & $10 / 14 / 2006$ & ND & ND \\
\hline EV009 & EV009-B-23375 & $4 / 2 / 2007$ & ND & ND \\
\hline EV009 & EV009-B-23410 & $7 / 26 / 2007$ & ND & ND \\
\hline EV009 & EV009-B-20152 & $7 / 25 / 2008$ & ND & ND \\
\hline EV009 & EV009-B-29736 & $8 / 28 / 2009$ & ND & ND \\
\hline EV009 & EV009-B-31735 & $7 / 28 / 2010$ & ND & ND \\
\hline EV009 & EV009-B-29767 & $7 / 29 / 2011$ & ND & ND \\
\hline EV009 & EV009-B-29787 & $7 / 12 / 2012$ & 0.38 & ND \\
\hline EV009 & EV009-B-29808 & $9 / 24 / 2013$ & ND & ND \\
\hline EV010 & EV010-B-19003 & $10 / 14 / 2006$ & ND & ND \\
\hline EV010 & EV010-B-23376 & $4 / 2 / 2007$ & ND & 0.85 \\
\hline EV010 & EV010-B-23412 & $7 / 26 / 2007$ & ND & ND \\
\hline EV010 & EV010-B-20153 & $7 / 25 / 2008$ & ND & ND \\
\hline EV010 & EV010-B-29737 & $8 / 28 / 2009$ & ND & ND \\
\hline EV010 & EV010-B-31734 & $7 / 28 / 2010$ & ND & ND \\
\hline EV010 & EV010-B-29766 & $7 / 29 / 2011$ & ND & ND \\
\hline EV010 & EV010-B-29786 & $7 / 12 / 2012$ & ND & 1.6 \\
\hline EV010 & EV010-B-29807 & 9/24/2013 & ND & ND \\
\hline EV011 & EV011-B-19005 & $10 / 14 / 2006$ & ND & ND \\
\hline EV012 & EV012-B-19007 & $10 / 14 / 2006$ & ND & 1.5 \\
\hline EV013 & EV013-B-19009 & $10 / 14 / 2006$ & ND & 1.2 \\
\hline EV013 & EV013-B-23377 & $4 / 2 / 2007$ & ND & ND \\
\hline EV013 & EV013-B-23414 & $7 / 26 / 2007$ & ND & ND \\
\hline EV013 & EV013-B-20136 & $7 / 25 / 2008$ & ND & ND \\
\hline EV013 & EV013-B-29720 & $8 / 28 / 2009$ & ND & ND \\
\hline EV013 & EV013-B-31720 & $7 / 28 / 2010$ & ND & ND \\
\hline EV013 & EV013-B-29750 & $7 / 29 / 2011$ & ND & ND \\
\hline EV013A & EV013A-B-29771 & $7 / 12 / 2012$ & 0.16 & ND \\
\hline EV013A & EV013A-B-29794 & $9 / 24 / 2013$ & ND & ND \\
\hline EV014 & EV014-B-23362 & $4 / 2 / 2007$ & ND & ND \\
\hline EV015 & EV015-B-23363 & $4 / 2 / 2007$ & ND & 0.84 \\
\hline EV016 & EV016-B-23364 & $4 / 2 / 2007$ & ND & ND \\
\hline EV016 & EV016-B-23386 & $7 / 26 / 2007$ & ND & ND \\
\hline EV016 & EV016-B-20140 & $7 / 25 / 2008$ & ND & ND \\
\hline EV016 & EV016-B-29724 & $8 / 28 / 2009$ & ND & ND \\
\hline EV016 & EV016-B-31723 & $7 / 28 / 2010$ & ND & ND \\
\hline EV016 & EV016-B-29754 & $7 / 29 / 2011$ & ND & 1.0 \\
\hline EV016 & EV016-B-29775 & $7 / 12 / 2012$ & ND & ND \\
\hline EV016 & EV016-B-29797 & 9/24/2013 & ND & ND \\
\hline EV017 & EV017-B-23367 & $4 / 2 / 2007$ & ND & ND \\
\hline EV017 & EV017-B-23394 & $7 / 26 / 2007$ & ND & ND \\
\hline
\end{tabular}


TABLE 3.6 (Cont.)

\begin{tabular}{|c|c|c|c|c|}
\hline \multirow[b]{2}{*}{ Location } & \multirow[b]{2}{*}{ Sample } & \multirow[b]{2}{*}{$\begin{array}{l}\text { Sample } \\
\text { Date }\end{array}$} & \multicolumn{2}{|c|}{ Concentration $(\mu \mathrm{g} / \mathrm{kg})$} \\
\hline & & & $\begin{array}{c}\text { Carbon } \\
\text { Tetrachloride }\end{array}$ & Chloroform \\
\hline EV017 & EV017-B-20144 & $7 / 25 / 2008$ & ND & ND \\
\hline EV017 & EV017-B-29728 & 8/28/2009 & ND & ND \\
\hline EV017 & EV017-B-31727 & $7 / 28 / 2010$ & ND & ND \\
\hline EV017 & EV017-B-29758 & 7/29/2011 & ND & ND \\
\hline EV017 & EV017-B-29778 & $7 / 12 / 2012$ & ND & 1.3 \\
\hline EV017 & EV017-B-29800 & 9/24/2013 & ND & ND \\
\hline EV018 & EV018-B-23368 & $4 / 2 / 2007$ & ND & ND \\
\hline EV018 & EV018-B-23398 & $7 / 26 / 2007$ & ND & ND \\
\hline EV018 & EV018-B-20145 & $7 / 25 / 2008$ & ND & ND \\
\hline EV018 & EV018-B-29729 & $8 / 28 / 2009$ & ND & ND \\
\hline EV018 & EV018-B-31728 & $7 / 28 / 2010$ & ND & ND \\
\hline EV018 & EV018-B-29759 & 7/29/2011 & ND & ND \\
\hline EV018 & EV018-B-29779 & 7/12/2012 & 0.11 & 1.2 \\
\hline EV018 & EV018-B-29801 & $9 / 24 / 2013$ & ND & ND \\
\hline EV019 & EV019-B-23371 & 4/2/2007 & ND & ND \\
\hline EV019 & EV019-B-23402 & $7 / 26 / 2007$ & ND & ND \\
\hline EV019 & EV019-B-20147 & $7 / 25 / 2008$ & ND & ND \\
\hline EV019 & EV019-B-29731 & 8/28/2009 & ND & ND \\
\hline EV019 & EV019-B-31730 & $7 / 28 / 2010$ & ND & ND \\
\hline EV019 & EV019-B-29761 & 7/29/2011 & ND & ND \\
\hline EV019 & EV019-B-29781 & $7 / 12 / 2012$ & ND & 2.6 \\
\hline EV019 & EV019-B-29803 & $9 / 24 / 2013$ & ND & ND \\
\hline EV020 & EV020-B-23373 & $4 / 2 / 2007$ & ND & 0.8 \\
\hline EV020 & EV020-B-23408 & $7 / 26 / 2007$ & ND & ND \\
\hline EV020 & EV020-B-20149 & $7 / 25 / 2008$ & ND & ND \\
\hline EV020 & EV020-B-29733 & 8/28/2009 & ND & ND \\
\hline EV020 & EV020-B-31733 & $7 / 28 / 2010$ & ND & ND \\
\hline EV020 & EV020-B-29763 & 7/29/2011 & ND & ND \\
\hline EV020 & EV020-B-29782 & 7/12/2012 & 0.69 & ND \\
\hline EV020 & EV020-B-29804 & $9 / 24 / 2013$ & ND & ND \\
\hline EV021 & EV021-B-23381 & $7 / 26 / 2007$ & ND & ND \\
\hline EV021 & EV021-B-20138 & $7 / 25 / 2008$ & ND & ND \\
\hline EV021 & EV021-B-29722 & 8/28/2009 & ND & ND \\
\hline EV021 & EV021-B-31722 & 7/28/2010 & ND & ND \\
\hline EV021 & EV021-B-29752 & 7/29/2011 & ND & 1.9 \\
\hline EV021 & EV021-B-29774 & $7 / 12 / 2012$ & ND & 2.1 \\
\hline EV021 & EV021-B-29796 & $9 / 24 / 2013$ & ND & ND \\
\hline EV022 & EV022-B-23388 & $7 / 26 / 2007$ & ND & ND \\
\hline EV022 & EV022-B-20141 & $7 / 25 / 2008$ & ND & ND \\
\hline EV022 & EV022-B-29725 & 8/28/2009 & ND & ND \\
\hline EV022 & EV022-B-31724 & $7 / 28 / 2010$ & ND & ND \\
\hline EV022 & EV022-B-29755 & 7/29/2011 & ND & 1.8 \\
\hline EV022 & EV022-B-29776 & $7 / 12 / 2012$ & ND & ND \\
\hline EV022 & EV022-B-29798 & 9/24/2013 & ND & ND \\
\hline
\end{tabular}


TABLE 3.6 (Cont.)

\begin{tabular}{|c|c|c|c|c|}
\hline \multirow[b]{2}{*}{ Location } & \multirow[b]{2}{*}{ Sample } & \multirow[b]{2}{*}{$\begin{array}{l}\text { Sample } \\
\text { Date }\end{array}$} & \multicolumn{2}{|c|}{ Concentration $(\mu \mathrm{g} / \mathrm{kg})$} \\
\hline & & & $\begin{array}{c}\text { Carbon } \\
\text { Tetrachloride }\end{array}$ & Chloroform \\
\hline EV023 & EV023-B-23390 & $7 / 26 / 2007$ & ND & ND \\
\hline EV023 & EV023-B-20139 & $7 / 25 / 2008$ & ND & ND \\
\hline EV023 & EV023-B-29723 & $8 / 28 / 2009$ & ND & ND \\
\hline EV023 & EV023-no sample & $7 / 29 / 2011$ & - & - \\
\hline EV024 & EV024-B-23396 & $7 / 26 / 2007$ & ND & ND \\
\hline EV024 & EV024-B-20143 & $7 / 25 / 2008$ & ND & ND \\
\hline EV024 & EV024-B-29727 & $8 / 28 / 2009$ & ND & ND \\
\hline EV024 & EV024-B-31726 & $7 / 28 / 2010$ & ND & ND \\
\hline EV024 & EV024-B-29757 & $7 / 29 / 2011$ & ND & ND \\
\hline EV024 & EV024-B-29777 & $7 / 12 / 2012$ & ND & ND \\
\hline EV024 & EV024-B-29799 & 9/24/2013 & ND & ND \\
\hline EV025 & EV025-B-23406 & $7 / 26 / 2007$ & ND & ND \\
\hline EV025 & EV025-B-20150 & $7 / 25 / 2008$ & ND & ND \\
\hline EV025 & EV025-B-29734 & $8 / 28 / 2009$ & ND & ND \\
\hline EV025 & EV025-B-31732 & $7 / 28 / 2010$ & ND & ND \\
\hline EV025 & EV025-B-29764 & $7 / 29 / 2011$ & ND & ND \\
\hline EV026 & EV026-B-29768 & $7 / 29 / 2011$ & ND & ND \\
\hline EV026 & EV026-B-29785 & $7 / 12 / 2012$ & ND & 1.1 \\
\hline EV026 & EV026-B-29806 & 9/24/2013 & ND & ND \\
\hline EV027 & EV027-B-29769 & $7 / 29 / 2011$ & ND & ND \\
\hline EV027 & EV027-B-29788 & $7 / 12 / 2012$ & Broken & Broken \\
\hline EV027 & EV027-B-29792 & 9/24/2013 & ND & ND \\
\hline EV028 & EV028-B-29747 & $7 / 29 / 2011$ & ND & ND \\
\hline EV028 & EV028-B-29772 & $7 / 12 / 2012$ & ND & 0.83 \\
\hline EV028 & EV028-B-29793 & $9 / 24 / 2013$ & ND & ND \\
\hline EV029 & EV029-B-29748 & $7 / 29 / 2011$ & ND & ND \\
\hline EV029 & EV029-B-29789 & $7 / 12 / 2012$ & ND & ND \\
\hline EV029 & EV029-B-29809 & 9/24/2013 & ND & ND \\
\hline EV030 & EV030-B-29749 & $7 / 29 / 2011$ & ND & ND \\
\hline EV030 & EV030-B-29791 & $7 / 12 / 2012$ & 0.94 & ND \\
\hline EV030 & EV030-B-29810 & 9/24/2013 & ND & ND \\
\hline EV031 & EV031-B-29770 & $7 / 29 / 2011$ & ND & ND \\
\hline EV031 & EV031-B-29790 & $7 / 12 / 2012$ & ND & ND \\
\hline EV031 & EV031-B-29811 & $9 / 24 / 2013$ & ND & ND \\
\hline EV032 & EV032-B-29784 & $7 / 12 / 2012$ & 0.52 & ND \\
\hline
\end{tabular}

a ND, Compound analyzed for but not detected at a level greater than or equal to the method detection limit $(<0.1 \mu \mathrm{g} / \mathrm{kg}$ for carbon tetrachloride or $<0.75$ $\mu \mathrm{g} / \mathrm{kg}$ for chloroform). 


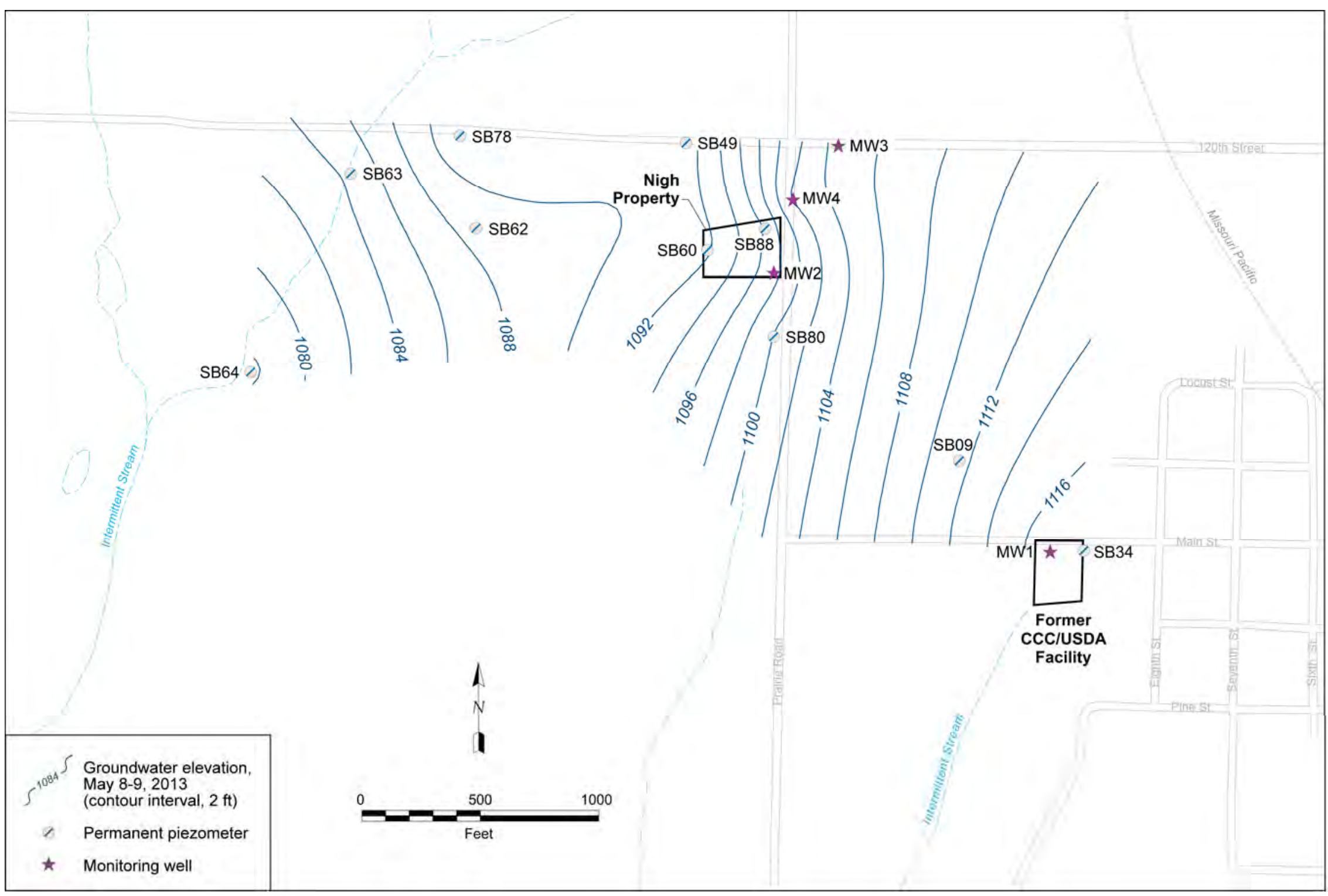

FIGURE 3.1 Potentiometric surface interpreted from groundwater levels measured on May 8-9, 2013. 

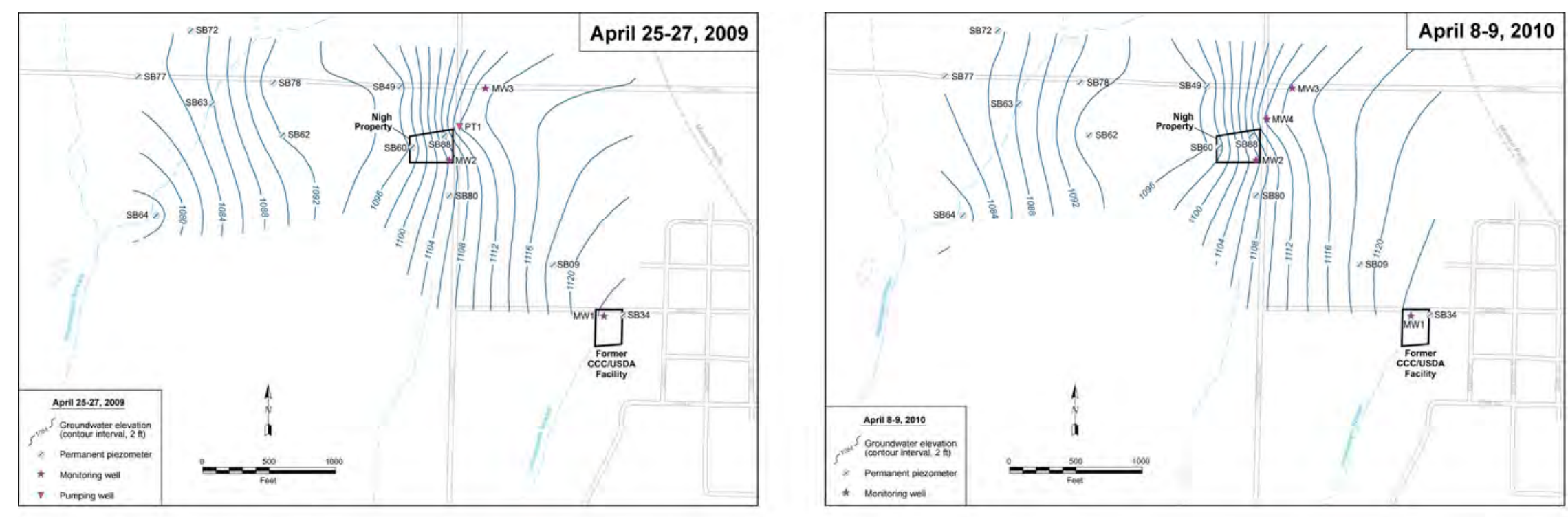

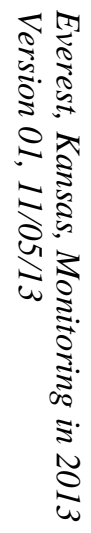
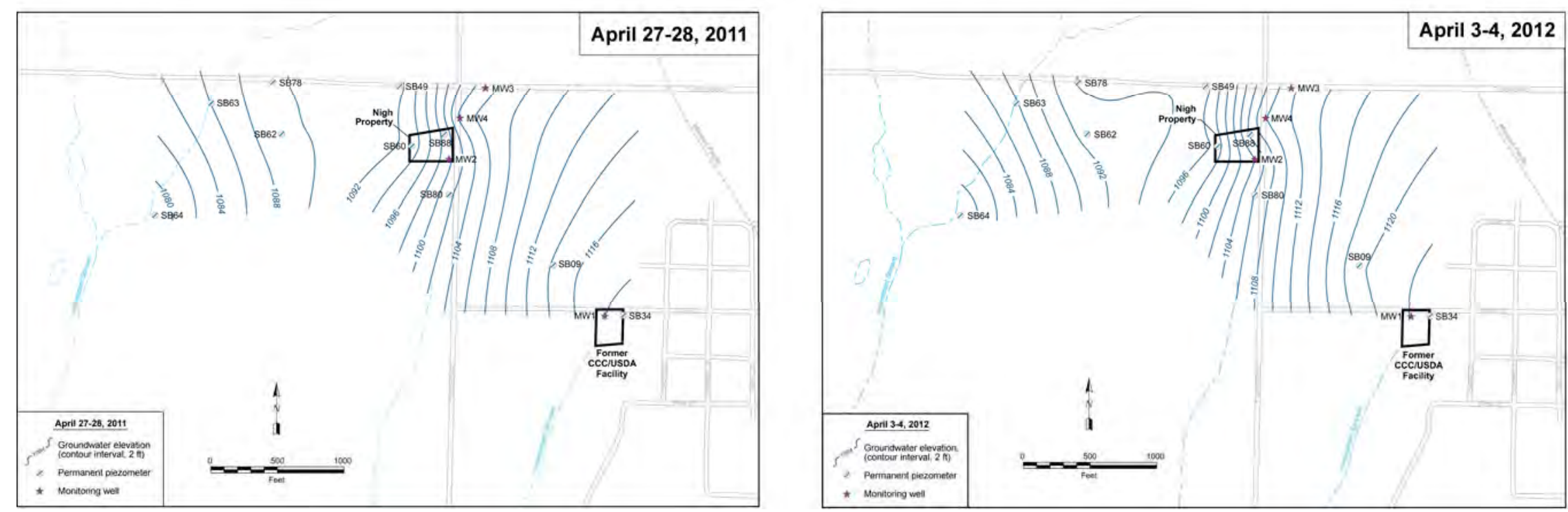

FIGURE 3.2 Historical potentiometric surface interpretations from groundwater levels measured in 2009, 2010, 2011 , and 2012. 


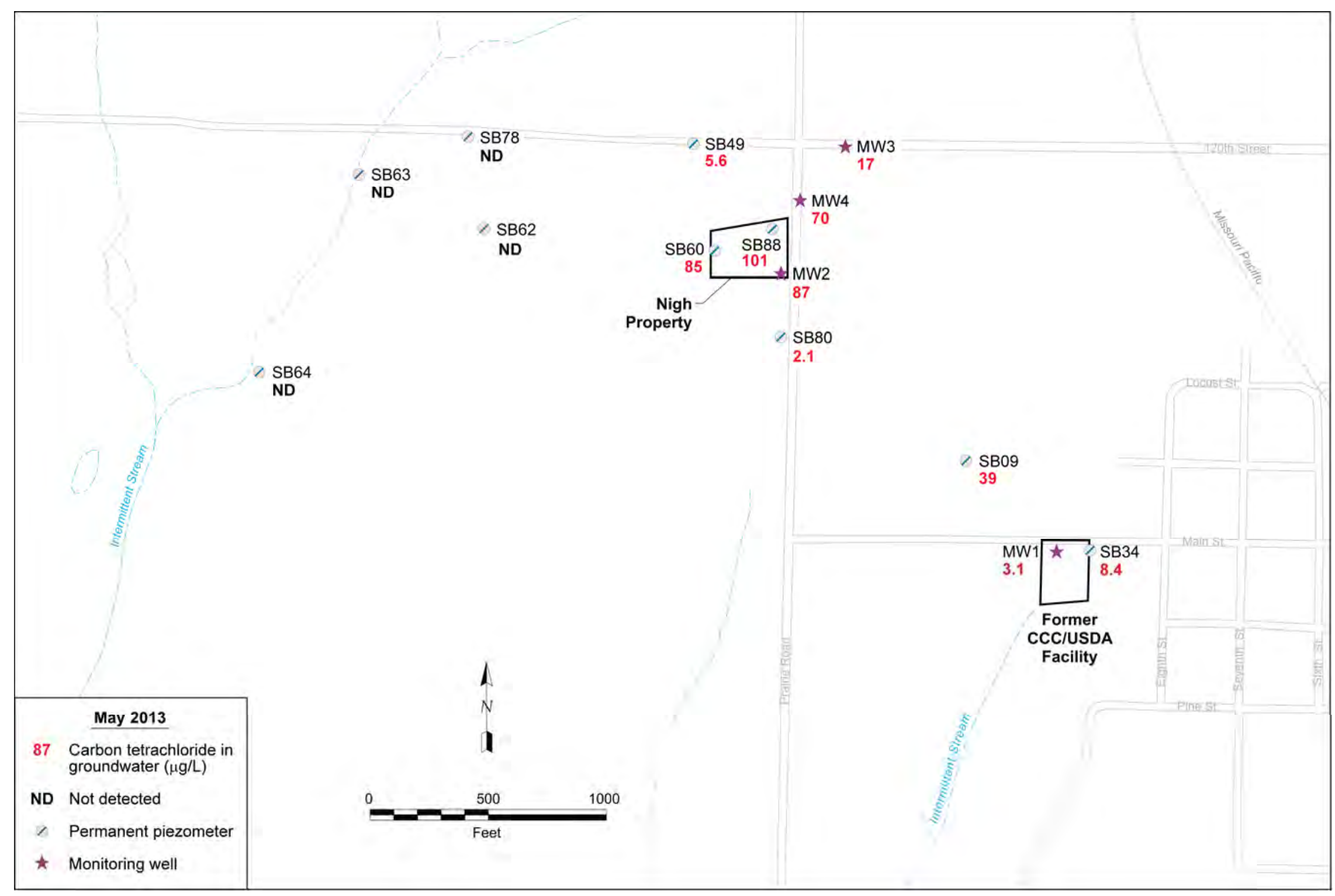

FIGURE 3.3 Lateral distribution of carbon tetrachloride in groundwater in May 2013. 


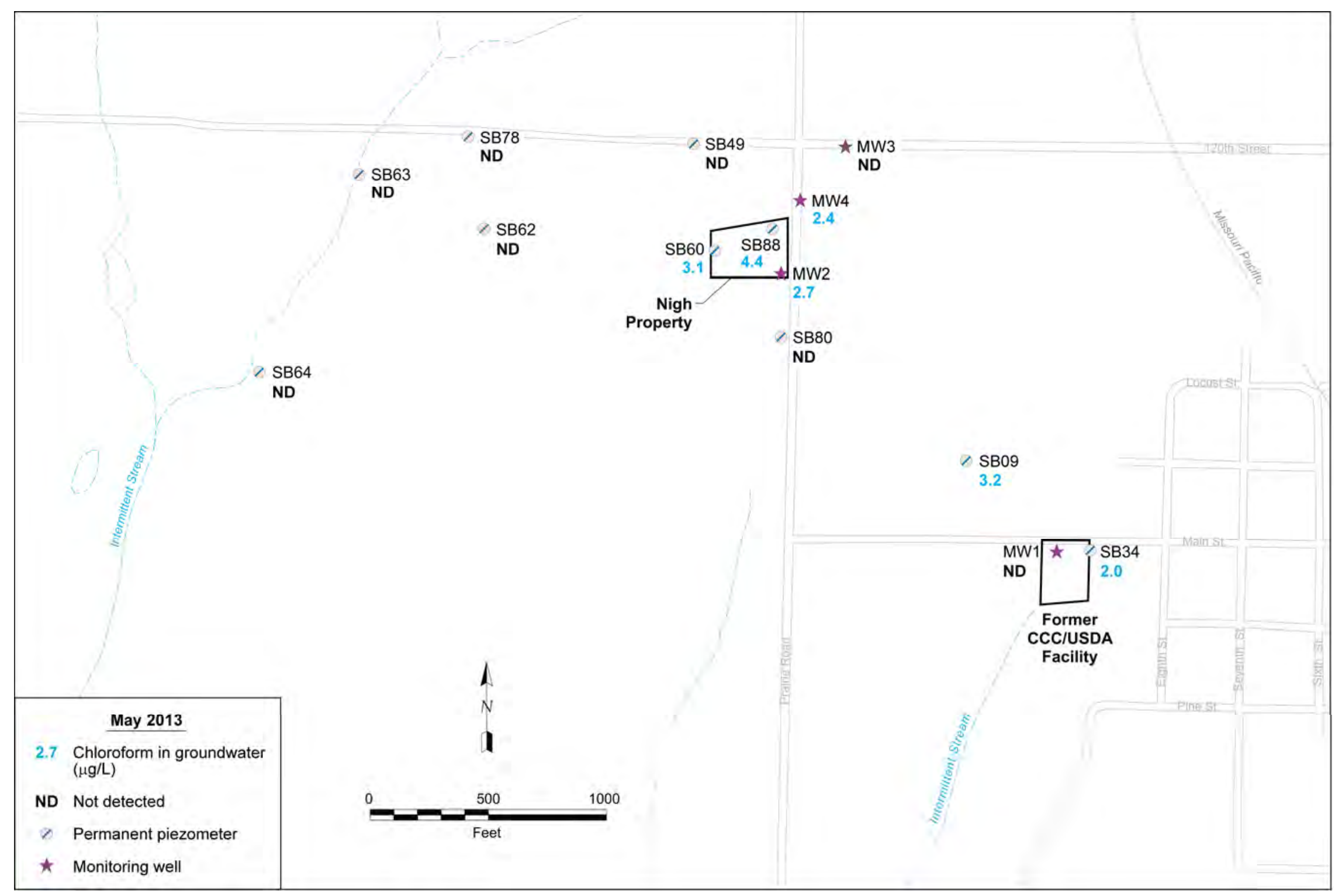

FIGURE 3.4 Lateral distribution of chloroform in groundwater in May 2013. 


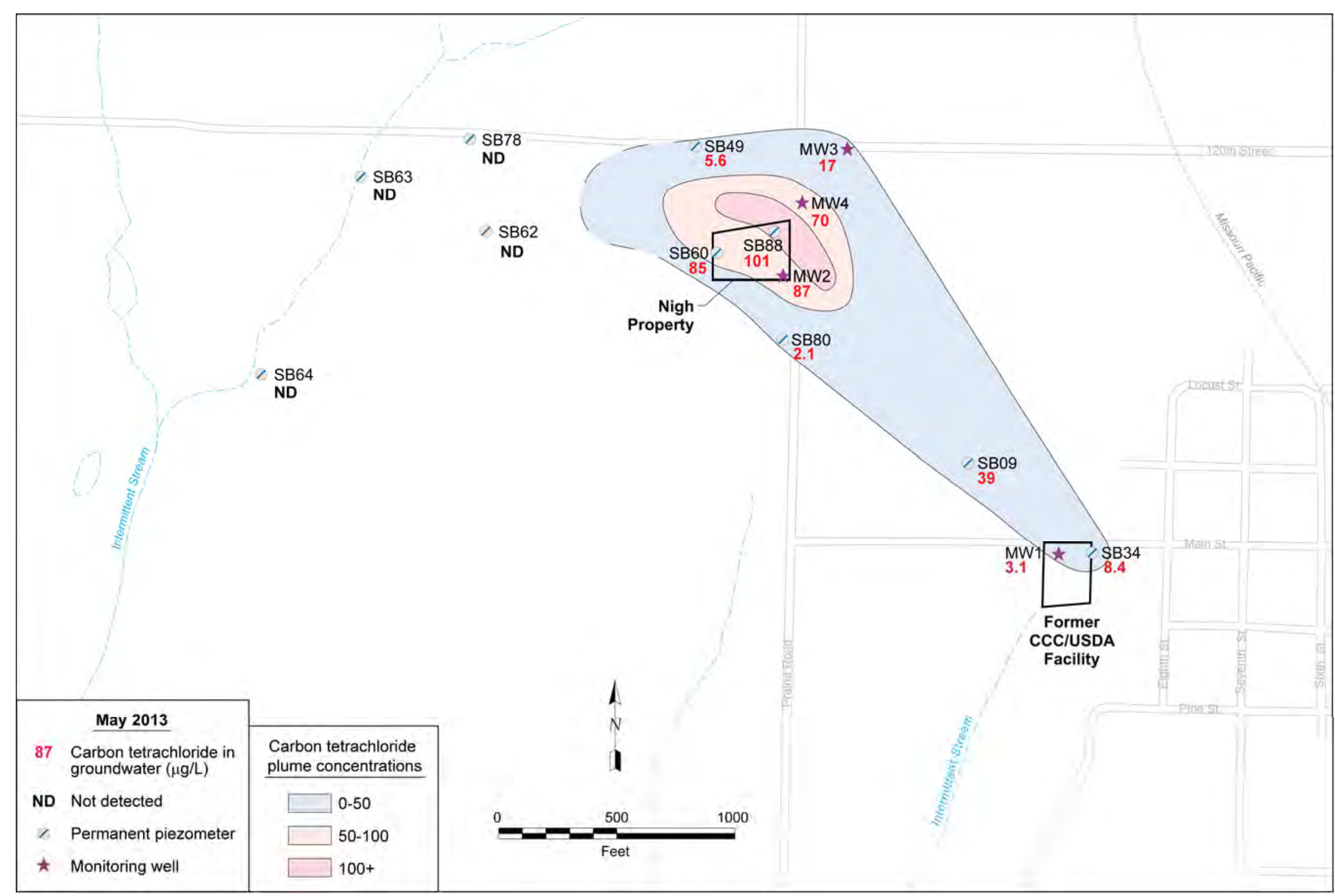

FIGURE 3.5 Interpreted carbon tetrachloride contaminant plume in groundwater at Everest, for data collected in May 2013. 


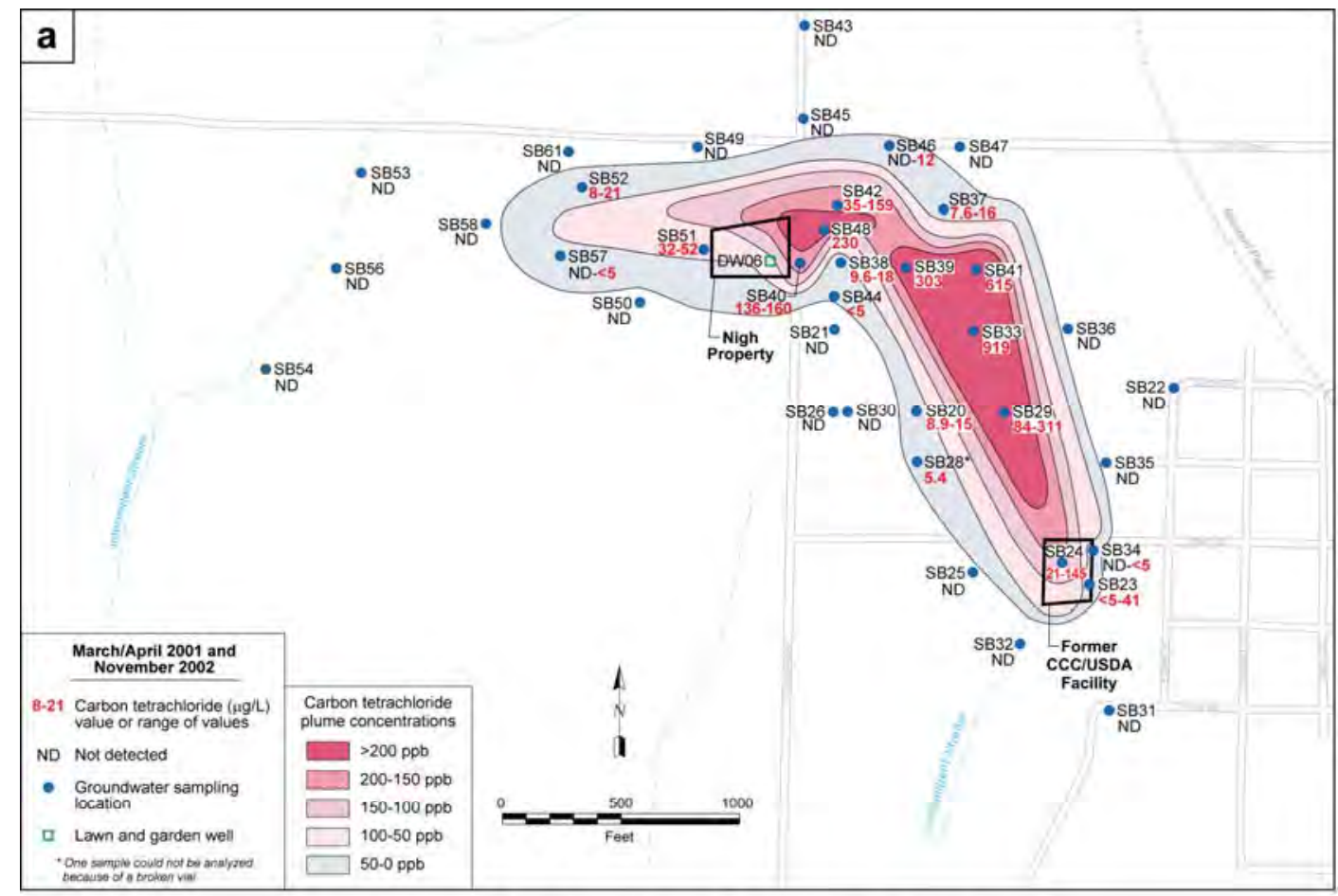

March/April 2001 and November 2002

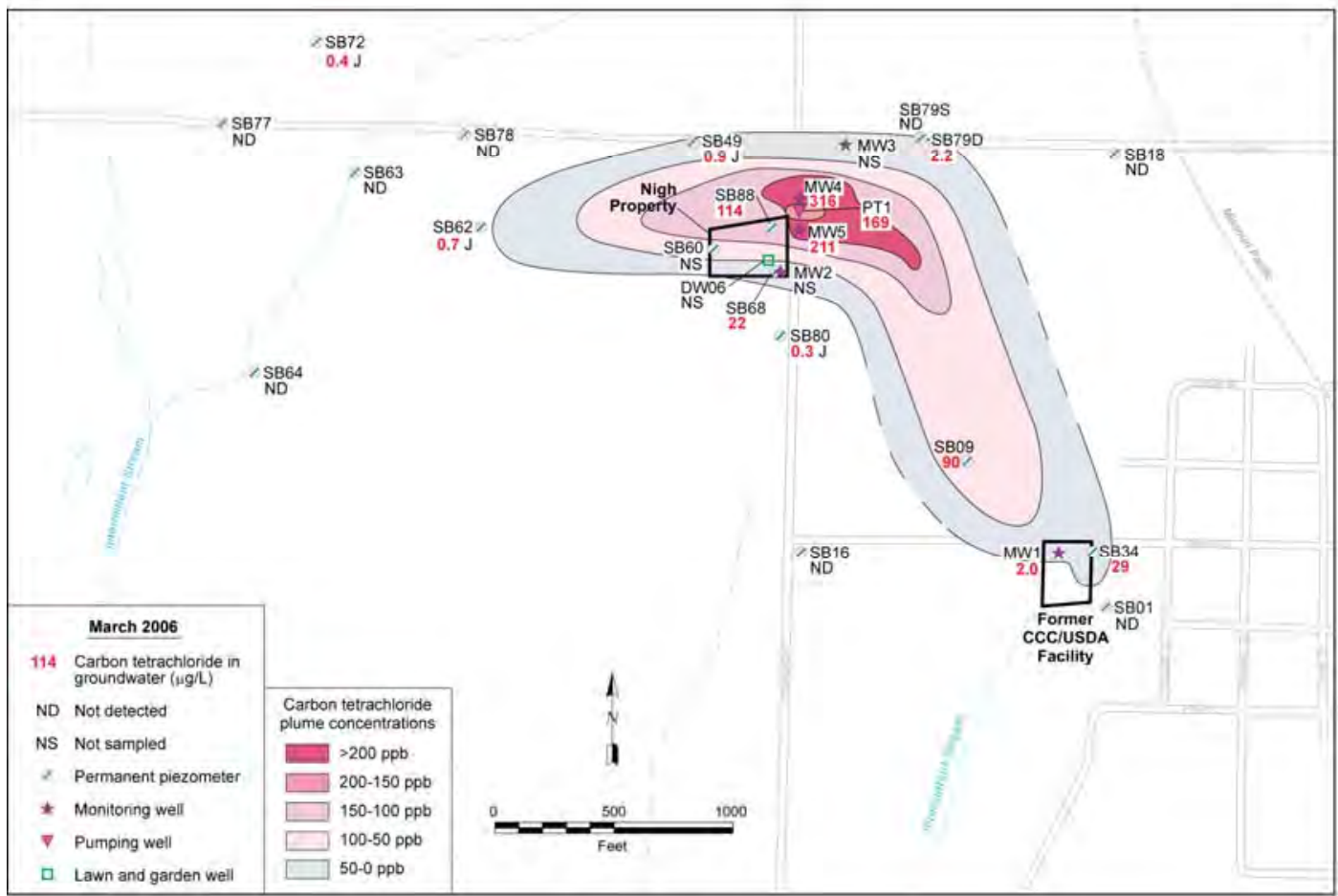

March 2006

FIGURE 3.6 Historical interpretations of the carbon tetrachloride contaminant plume in groundwater at Everest, for data collected in (a) 2001-2002 and 2006, (b) 2008 and 2010, (c) 2011 and 2012, and (d) 2001-2002 and 2013. 
Everest, Kansas, Monitoring in 2013

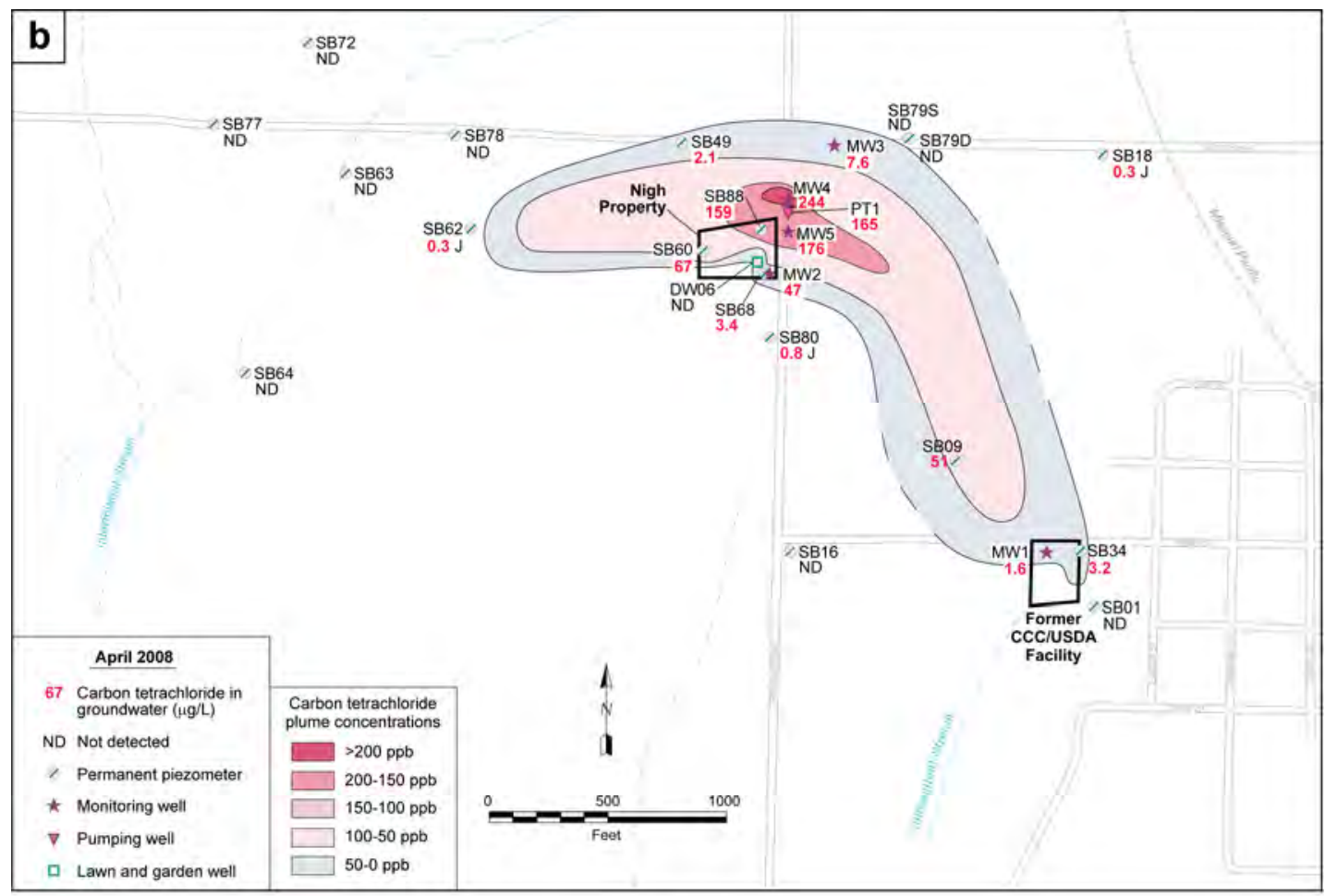

April 2008

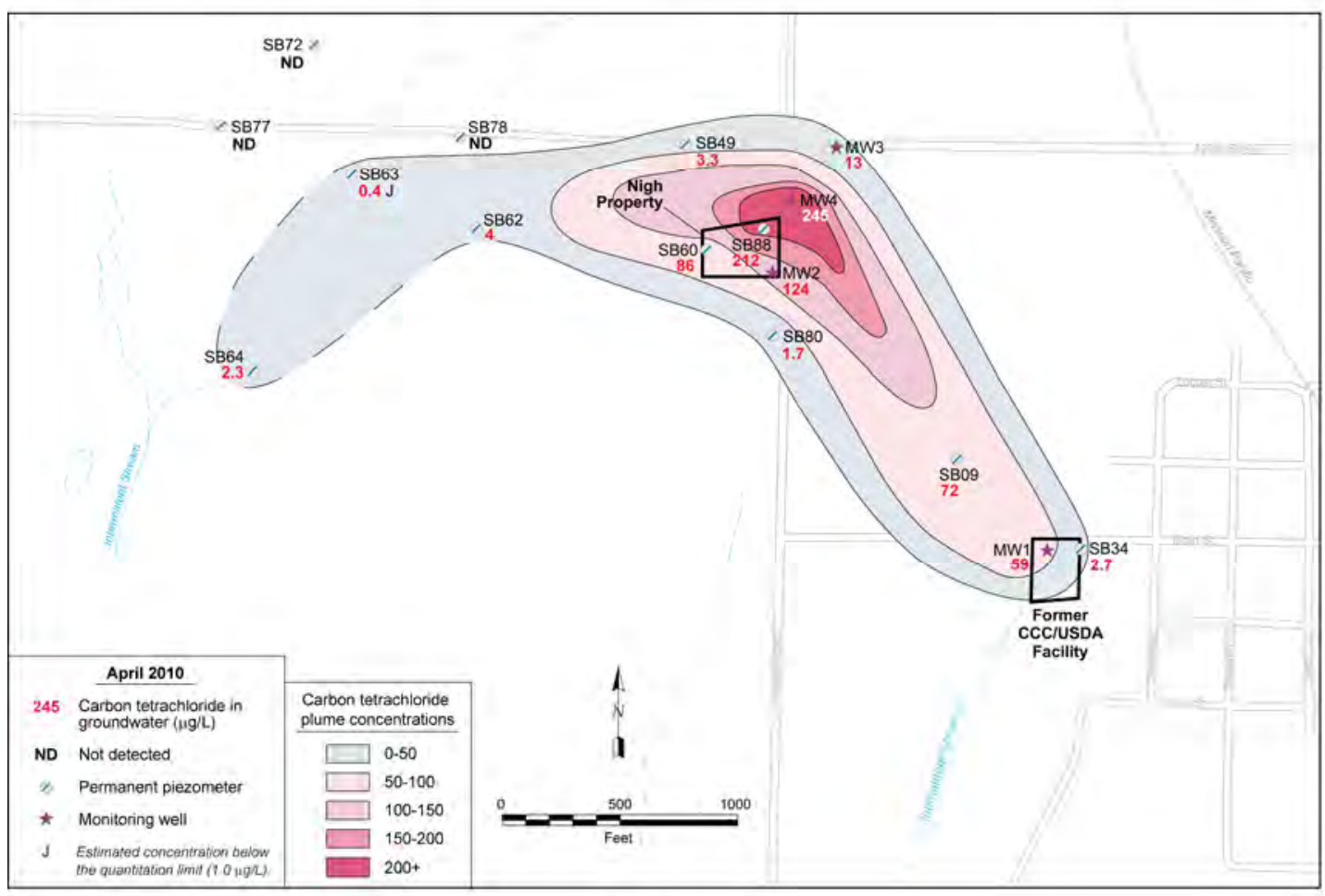

April 2010

FIGURE 3.6 (Cont.) 
Everest, Kansas, Monitoring in 2013

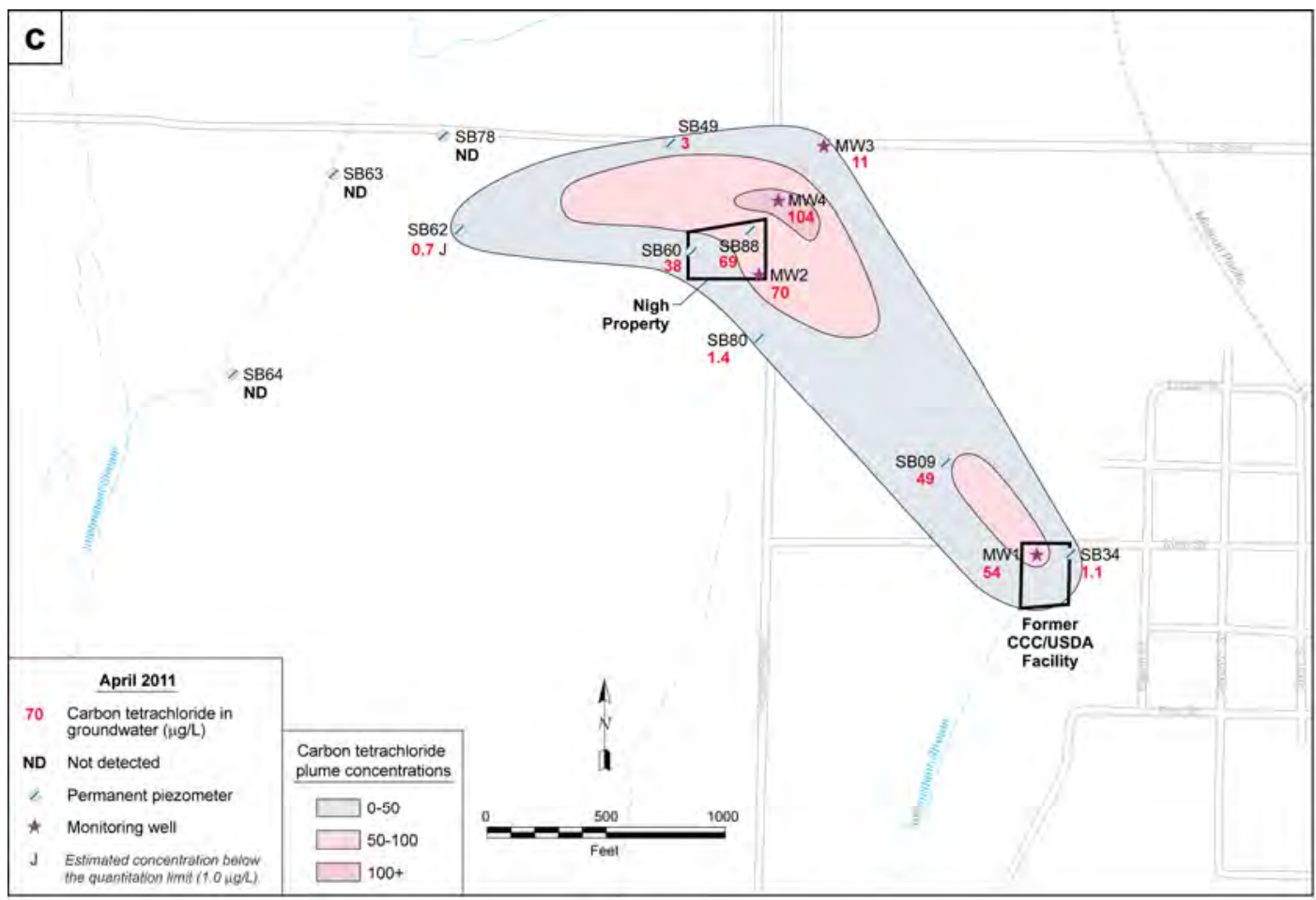

April 2011

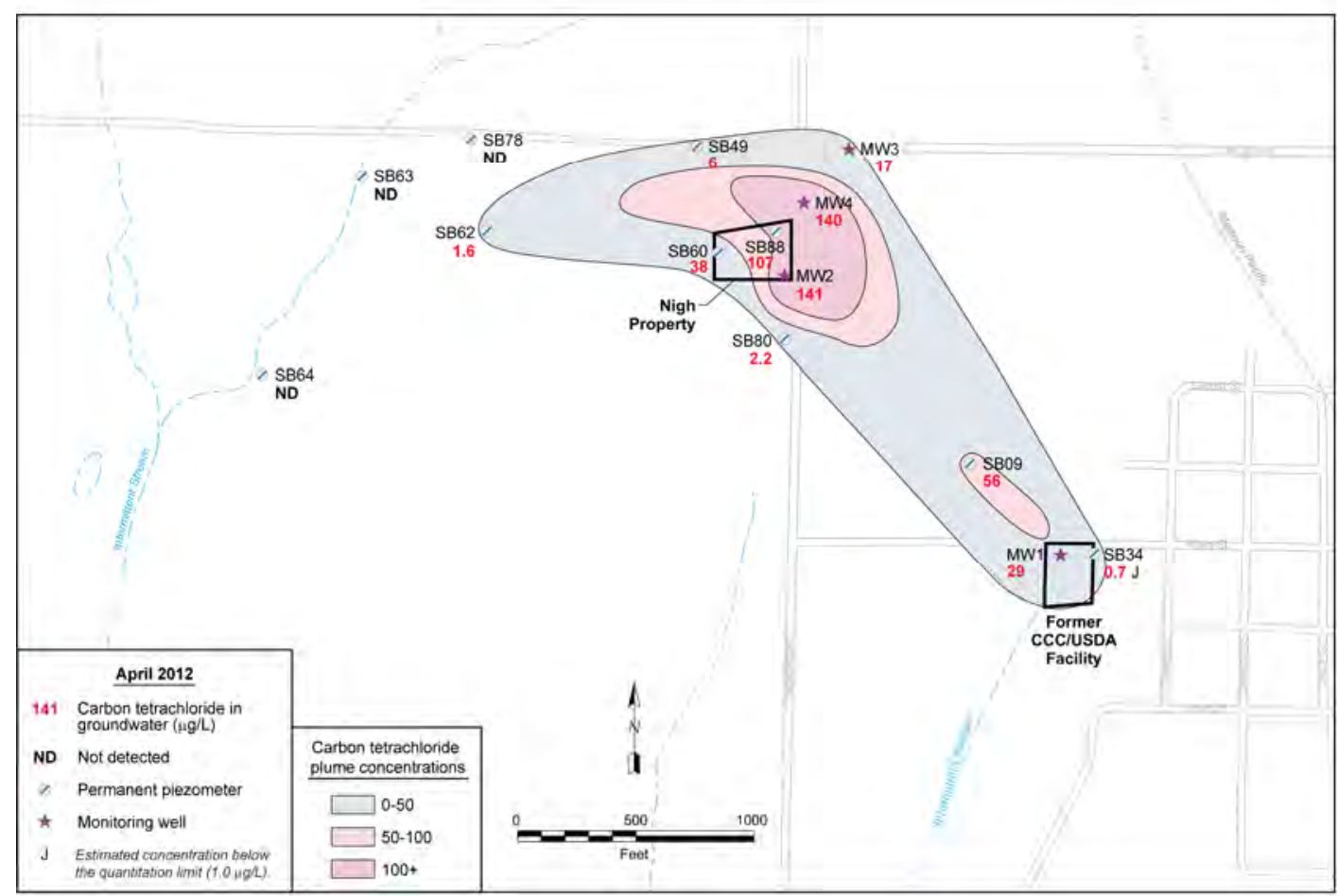

April 2012

FIGURE 3.6 (Cont.) 
Everest, Kansas, Monitoring in 2013

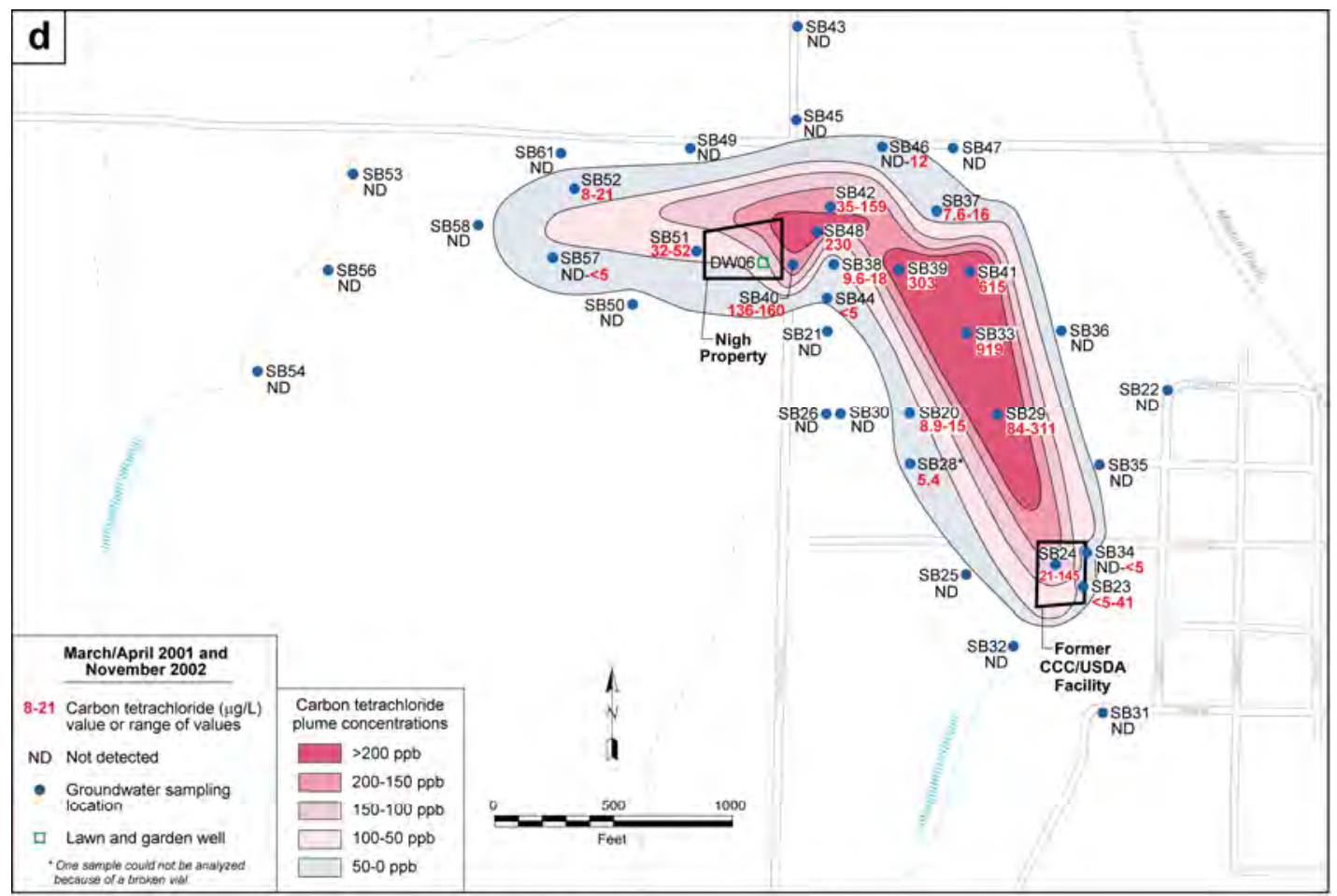

March/April 2001 and November 2002

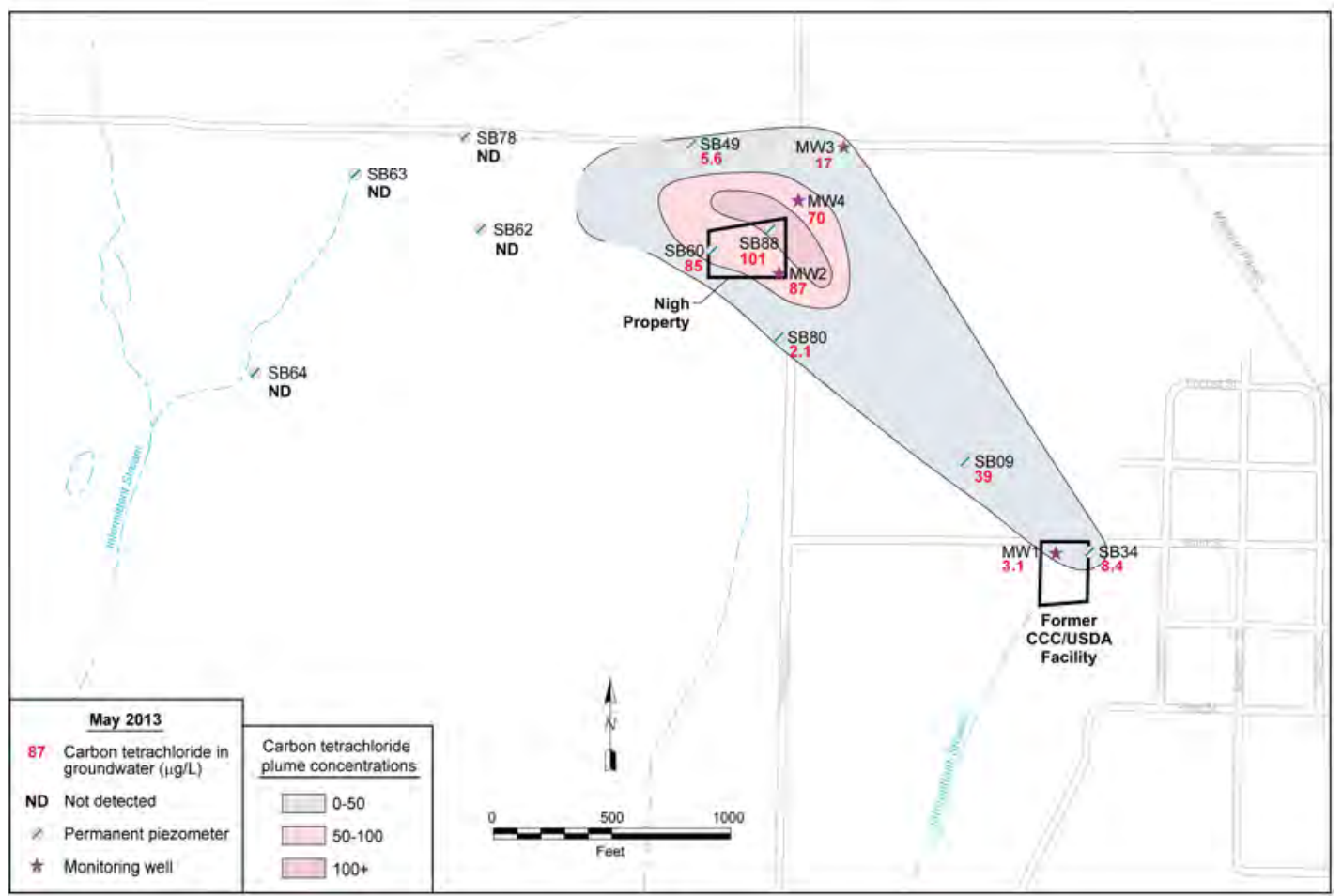

May 2013

FIGURE 3.6 (Cont.) 


\section{Conclusions and Recommendations}

\subsection{Conclusions}

The findings of the 2013 monitoring at Everest support the following conclusions:

- Manual measurements of groundwater levels during annual monitoring in 2009-2013 and automatically recorded data in 2002-2010 have consistently indicated an initial direction of groundwater flow from the former CCC/USDA facility to the north-northwest toward the Nigh (formerly Gale) property, then west-southwest from the Nigh property toward the intermittent creek that lies west of the former CCC/USDA facility and the Nigh property.

- The accumulated data demonstrate that the area of the carbon tetrachloride plume with concentrations $>200 \mu \mathrm{g} / \mathrm{L}$ has decreased markedly over time, indicating a decreasing trend in contaminant levels. During annual monitoring in 2010-2013, maximum contaminant levels decreased by approximately 50\% at SB88 and 70\% at MW4, near the identified source area on the Nigh (formerly Gale) property.

- The results of the May 2013 monitoring event continue to support the interpretation, based on observations in 2001-2012, that the migration rate for contamination in groundwater toward the intermittent creek is very slow.

- Sampling of surface water at five locations in the intermittent creek west of the former CCC/USDA facility and the Nigh property (downgradient of the identified carbon tetrachloride plume) has shown no detection of carbon tetrachloride or chloroform in 2009-2013. These observations indicate that the carbon tetrachloride contamination identified at Everest has not impacted the surface waters of the intermittent creek.

- No carbon tetrachloride was found in the vegetation samples collected. The present results are consistent with the analyses of vegetation reported in 20062011. 


\subsection{Recommendations}

The findings of the annual monitoring at Everest in 2013 support the following recommendations:

- In view of the slow migration of contamination in groundwater that has been documented since 2001 and the observed decrease in contaminant concentrations, the present KDHE-approved annual frequency for monitoring of the groundwater and surface waters should continue.

- Surface water and vegetation sampling during the spring and summer of 2014 should also continue. 


\section{References}

Alvarado, J.S., and C.M. Rose, 2004, "Static Headspace Analysis of Volatile Organic Compounds in Soil and Vegetation Samples for Site Characterization,” Talanta 62:17-23.

Argonne, 2000, Final Phase I Work Plan: QuickSite ${ }^{\circledR}$ Investigation, Everest, Kansas, prepared for the Commodity Credit Corporation, U.S. Department of Agriculture, by Argonne National Laboratory, Argonne, Illinois, May (revised March 2003).

Argonne, 2001, Final Phase I Report and Phase II Work Plan: QuickSite ${ }^{\circledR}$ Investigation, Everest, Kansas, ANL/ER/TR-01/004, prepared for the Commodity Credit Corporation, U.S. Department of Agriculture, Washington, D.C., by Argonne National Laboratory, Argonne, Illinois, August (revised March 2003).

Argonne, 2002, Final Master Work Plan: Environmental Investigations at Former CCC/USDA Facilities in Kansas, 2002 Revision, ANL/ER/TR-02/004, prepared for the Commodity Credit Corporation, U.S. Department of Agriculture, Washington, D.C., by Argonne National Laboratory, Argonne, Illinois, December.

Argonne, 2003, Final Phase II Report: QuickSite ${ }^{\circledR}$ Investigation, Everest, Kansas, ANL/ER/TR03/003, prepared for the Commodity Credit Corporation, U.S. Department of Agriculture, Washington, D.C., by Argonne National Laboratory, Argonne, Illinois, September.

Argonne, 2005, Scoping Memo for the Discussion of Potential Remedial Options for the Groundwater Contamination Site at Everest, Kansas, ANL/ER/AGEM/CHRON-883, prepared for the Commodity Credit Corporation, U.S. Department of Agriculture, Washington, D.C., by Argonne National Laboratory, Argonne, Illinois, September.

Argonne, 2006a, Final Report: Phase III Targeted Investigation, Everest, Kansas, ANL/ER/TR04/004, prepared for the Commodity Credit Corporation, U.S. Department of Agriculture, Washington, D.C., by Argonne National Laboratory, Argonne, Illinois, January.

Argonne, 2006b, 2005 Cross Section Analysis and Recommendations for Further Studies at Everest, Kansas, ANL/EVS/AGEM/TR-05-02, prepared for the Commodity Credit Corporation, 
U.S. Department of Agriculture, Washington, D.C., by Argonne National Laboratory, Argonne, Illinois, January.

Argonne, 2006c, Plan for Proposed Aquifer Hydraulic Testing and Groundwater Sampling at Everest, Kansas, in January-February 2006, ANL/EVS/AGEM/TR-06-01, prepared for the Commodity Credit Corporation, U.S. Department of Agriculture, Washington, D.C., by Argonne National Laboratory, Argonne, Illinois, January.

Argonne, 2006d, Final Report: Results of Aquifer Pumping and Groundwater Sampling at Everest, Kansas, in January-March 2006, ANL/EVS/AGEM/TR-06-05, prepared for the Commodity Credit Corporation, U.S. Department of Agriculture, Washington, D.C., by Argonne National Laboratory, Argonne, Illinois, September.

Argonne, 2007a, Recommendations for Remedial Action at Everest, Kansas, ANL/EVS/AGEM/CHRON-1025, prepared for the Commodity Credit Corporation, U.S. Department of Agriculture, Washington, D.C., by Argonne National Laboratory, Argonne, Illinois, February.

Argonne, 2007b, Recommendations for New Monitoring Wells at Everest, Kansas, ANL/EVS/AGEM/CHRON-1041, prepared for the Commodity Credit Corporation, U.S. Department of Agriculture, Washington, D.C., by Argonne National Laboratory, Argonne, Illinois, May.

Argonne, 2008, Results of Groundwater Monitoring at Everest, Kansas, in April 2008, ANL/EVS/AGEM/TR-08-16, prepared for the Commodity Credit Corporation, U.S. Department of Agriculture, Washington, D.C., by Argonne National Laboratory, Argonne, Illinois, September.

Argonne, 2009, Monitoring Plan for Everest, Kansas, ANL/EVS/AGEM/CHRON-1260, prepared for the Commodity Credit Corporation, U.S. Department of Agriculture, Washington, D.C., by Argonne National Laboratory, Argonne, Illinois, March. 
Argonne, 2010a, Results of Groundwater Monitoring and Vegetation Sampling at Everest, Kansas, in 2009, ANL/EVS/AGEM/TR-10-03, prepared for the Commodity Credit Corporation, U.S. Department of Agriculture, Washington, D.C., by Argonne National Laboratory, Argonne, Illinois, April.

Argonne, 2010b, Final Work Plan: Indoor Air and Ambient Air Sampling near the Former CCC/USDA Grain Storage Facility in Everest, Kansas, ANL/EVS/AGEM/CHRON-1341, prepared for the Commodity Credit Corporation, U.S. Department of Agriculture, Washington, D.C., by Argonne National Laboratory, Argonne, Illinois, April.

Argonne, 2011a, Annual Report of Groundwater Monitoring at Everest, Kansas, in 2010, ANL/EVS/AGEM/TR-11-03, prepared for the Commodity Credit Corporation, U.S. Department of Agriculture, Washington, D.C., by Argonne National Laboratory, Argonne, Illinois, March.

Argonne, 2011b, Annual Report of Groundwater Monitoring at Everest, Kansas, in 2011, ANL/EVS/AGEM/TR-11-11, prepared for the Commodity Credit Corporation, U.S. Department of Agriculture, Washington, D.C., by Argonne National Laboratory, Argonne, Illinois, December.

Argonne, 2013, Annual Report of Groundwater Monitoring at Everest, Kansas, in 2012, ANL/EVS/AGEM/TR-12-13, prepared for the Commodity Credit Corporation, U.S. Department of Agriculture, Washington, D.C., by Argonne National Laboratory, Argonne, Illinois, July.

EPA, 1995, Method 524.2: Measurement of Purgeable Organic Compounds in Water by Capillary Column Gas Chromatography/Mass Spectrometry, Revision 4.1, edited by J.W. Munch, National Exposure Research Laboratory, Office of Research and Development, U.S. Environmental Protection Agency, Cincinnati, Ohio.

KDHE, 1998a, Pre-CERCLIS Site Reconnaissance and Evaluation: Everest USDA/CCC Site, Everest, Kansas, KDHE ID P4-007-70591, Kansas Department of Health and Environment, Bureau of Environmental Remediation, Pre-Remedial Unit, Remedial Section, Topeka, Kansas, August 28.

KDHE, 1998b, Pre-CERCLIS Site Reconnaissance and Evaluation: Everest East USDA/CCC Site, Everest, Kansas, KDHE ID P4-007-70633, Kansas Department of Health and Environment, 
Bureau of Environmental Remediation, Pre-Remedial Unit, Remedial Section, Topeka, Kansas, August.

KDHE, 2006, letter from C. Carey (Bureau of Environmental Remediation, Kansas Department of Health and Environment, Topeka, Kansas) to C. Roe (Commodity Credit Corporation, U.S. Department of Agriculture, Washington, D.C.), regarding report of aquifer pumping and groundwater sampling in January-March 2006, August 11.

KDHE, 2009, letter from E. Finzer (Bureau of Environmental Remediation, Kansas Department of Health and Environment, Topeka, Kansas) to C. Roe (Commodity Credit Corporation, U.S. Department of Agriculture, Washington, D.C.), regarding the plan for annual monitoring at Everest, May 12.

KDHE, 2010, letter from C. Carey (Bureau of Environmental Remediation, Kansas Department of Health and Environment, Topeka, Kansas) to C. Roe (Commodity Credit Corporation, U.S. Department of Agriculture, Washington, D.C.), regarding the report of groundwater monitoring and vegetation sampling at Everest in 2009, September 7.

Puls, R.W., and Barcelona, M.J., 1996, "Low-Flow (Minimal Drawdown) Ground-Water Sampling Procedures,” EPA/540/S-95/504, in Ground Water Issue, Superfund Technology Support Center for Ground Water, National Risk Management Research Laboratory, Ada, Oklahoma, April (www.epa.gov/tio/tsp/download/lwflw2a.pdf).

Yeskis, D., and B. Zavala, 2002, Ground-Water Sampling Guidelines for Superfund and RCRA Project Managers: Ground Water Forum Issue Paper, EPA 542-S-02-001, Technology Innovative Office, Office of Solid Waste and Emergency Response, U.S. Environmental Protection Agency, Washington, D.C., May (http://www.epa.gov/tio/tsp/download/ gw_sampling_guide.pdf).

USGS, 1979, Everest Quadrangle, Kansas, 7.5 Minute Series, U.S. Geological Survey, Washington, D.C. 


\section{Appendix A:}

Sequence of Sampling Activities during the 2013 Annual Monitoring Event at Everest 
TABLE A.1 Sequence of sampling activities during annual monitoring in 2013.

\begin{tabular}{|c|c|c|c|c|c|c|c|c|c|}
\hline \multicolumn{2}{|c|}{$\begin{array}{l}\text { Sample } \\
\text { Date and Time }\end{array}$} & \multirow{2}{*}{$\begin{array}{c}\text { Sample } \\
\text { EVDIH2O-W-29841 }\end{array}$} & \multirow{2}{*}{$\begin{array}{c}\text { Location } \\
\text { QC }\end{array}$} & \multirow{2}{*}{$\begin{array}{c}\text { Sample } \\
\text { Type }^{a} \\
\text { FB }\end{array}$} & \multirow{2}{*}{$\begin{array}{l}\text { Sample } \\
\text { Matrix }\end{array}$} & \multirow{2}{*}{$\begin{array}{c}\text { Depth or } \\
\text { Height } \\
\text { (ft BGL or } \mathrm{ft})^{\mathrm{c}}\end{array}$} & \multirow{2}{*}{$\begin{array}{c}\text { Chain of } \\
\text { Custody } \\
6890\end{array}$} & \multirow{2}{*}{$\begin{array}{l}\begin{array}{c}\text { Shipping } \\
\text { Date }\end{array} \\
5 / 9 / 2013\end{array}$} & \multirow[t]{2}{*}{ Sample Description } \\
\hline $5 / 8 / 13$ & $12: 00$ & & & & & & & & \\
\hline $5 / 8 / 13$ & $12: 00$ & EVQCTB-W-29842 ${ }^{\mathrm{d}}$ & QC & TB & WQC & & 6890 & $5 / 9 / 2013$ & $\begin{array}{l}\text { Trip blank sent to the AGEM Laboratory for } \\
\text { organic analysis with water samples listed on } \\
\text { chain-of-custody form (COC) } 6890 \text {. }\end{array}$ \\
\hline $5 / 8 / 13$ & $12: 00$ & EVQCTB-W-29842VER ${ }^{d}$ & $\mathrm{QC}$ & VER & WQC & & 6889 & $5 / 9 / 2013$ & Verification sample sent to Test America. \\
\hline $5 / 8 / 13$ & $13: 25$ & EVO05-W-29820DUP $^{d}$ & EVO05 & DUP-L & WS & & 6748 & $5 / 8 / 2013$ & Duplicate laboratory analysis. \\
\hline $5 / 8 / 13$ & $13: 25$ & EV005-W-29820 & EV005 & $\mathrm{N}$ & WS & & 6748 & $5 / 8 / 2013$ & Collected from flowing water in the creek. \\
\hline $5 / 8 / 13$ & $13: 30$ & EV006-W-29821 & EV006 & $\mathrm{N}$ & WS & & 6748 & $5 / 8 / 2013$ & Collected from flowing water in the creek. \\
\hline $5 / 8 / 13$ & 13:35 & EV008-W-29822 & EV008 & $\mathrm{N}$ & WS & & 6748 & $5 / 8 / 2013$ & Collected from flowing water in the creek. \\
\hline $5 / 8 / 13$ & 13:55 & EV003-W-29819 & EV003 & $\mathrm{N}$ & WS & & 6748 & $5 / 8 / 2013$ & Collected from flowing water in the creek. \\
\hline $5 / 8 / 13$ & $14: 00$ & EV001-W-29818 & EV001 & $\mathrm{N}$ & WS & & 6748 & $5 / 8 / 2013$ & Collected from flowing water in the creek. \\
\hline $5 / 8 / 13$ & $15: 16$ & EVMW1-W-29823DUP $^{d}$ & MW1 & DUP-L & WG & $41-51$ & 6748 & $5 / 8 / 2013$ & Duplicate laboratory analysis. \\
\hline $5 / 8 / 13$ & $15: 16$ & EVMW1-W-29823 & MW1 & $\mathrm{N}$ & WG & $41-51$ & 6748 & $5 / 8 / 2013$ & $\begin{array}{l}\text { Depth to water }=10.15 \mathrm{ft} \text {. Depth of } 4 \text {-in. well }= \\
51 \mathrm{ft} . \text { Sample collected by using a low-flow } \\
\text { bladder pump positioned at } 46 \mathrm{ft} \text { after purging } \\
\text { of } 6.2 \mathrm{~L} \text {. }\end{array}$ \\
\hline $5 / 8 / 13$ & $15: 16$ & EVMW1-W-29823VER $^{d}$ & MW1 & VER & WG & $41-51$ & 6889 & $5 / 9 / 2013$ & Verification sample sent to Test America. \\
\hline $5 / 8 / 13$ & $16: 20$ & EVSB34-W-29828 & SB34 & $\mathrm{N}$ & WG & $46-53$ & 6748 & $5 / 8 / 2013$ & $\begin{array}{l}\text { Depth to water }=14.81 \mathrm{ft} \text {. Depth of } 1 \text {-in. well }= \\
53 \mathrm{ft} . \text { Sample collected by using a low-flow } \\
\text { bladder pump positioned at } 49.5 \text { after purging } \\
\text { of } 2 \mathrm{~L} \text {. }\end{array}$ \\
\hline $5 / 8 / 13$ & $16: 30$ & EVQCIR-W-29840 ${ }^{\mathrm{d}}$ & QC & RI & WQC & & 6748 & $5 / 8 / 2013$ & $\begin{array}{l}\text { Rinsate of decontaminated flow line after } \\
\text { collection of sample EVSB34-W-29828. }\end{array}$ \\
\hline $5 / 8 / 13$ & $16: 40$ & EVSB64-W-29833 & SB64 & $\mathrm{N}$ & WG & $22-27$ & 6748 & $5 / 8 / 2013$ & $\begin{array}{l}\text { Depth to water }=18.29 \mathrm{ft} \text {. Depth of } 1 \text {-in. well = } \\
27 \mathrm{ft} \text {. Sample collected by using a low-flow } \\
\text { bladder pump positioned at } 24.5 \mathrm{ft} \text { after } \\
\text { purging of } 3 \mathrm{~L} \text {. }\end{array}$ \\
\hline $5 / 8 / 13$ & $17: 34$ & EVMW3DUP-W-29837 ${ }^{d}$ & MW3 & DUP-F & WG & $56.5-71.5$ & 6748 & $5 / 8 / 2013$ & Field replicate. \\
\hline $5 / 8 / 13$ & $17: 34$ & EVMW3DUP-W-29837DUP ${ }^{d}$ & MW3 & DUP-L & WG & $56.5-71.5$ & 6748 & $5 / 8 / 2013$ & Duplicate laboratory analysis. \\
\hline $5 / 8 / 13$ & $17: 34$ & EVMW3-W-29825 & MW3 & $\mathrm{N}$ & WG & $56.5-71.5$ & 6748 & $5 / 8 / 2013$ & $\begin{array}{l}\text { Depth to water }=41.0 \mathrm{ft} \text {. Depth of } 2 \text {-in. well }= \\
70.5 \mathrm{ft} \text {. Sample collected by using a low-flow } \\
\text { bladder pump positioned at } 64 \mathrm{ft} \text { after purging } \\
\text { of } 8 \mathrm{~L} \text {. }\end{array}$ \\
\hline $5 / 8 / 13$ & $17: 39$ & EVSB62-W-29831 & SB62 & $\mathrm{N}$ & WG & $33-41$ & 6748 & $5 / 8 / 2013$ & $\begin{array}{l}\text { Depth to water }=30.15 \mathrm{ft} \text {. Depth of } 1 \text {-in. well }= \\
41 \mathrm{ft} \text {. Sample collected by using a low-flow } \\
\text { bladder pump positioned at } 37 \mathrm{ft} \text { after purging } \\
\text { of } 3 \mathrm{~L} \text {. }\end{array}$ \\
\hline $5 / 8 / 13$ & $18: 15$ & EVQCIR-W-29839 ${ }^{\mathrm{d}}$ & QC & RI & WQC & & 6748 & $5 / 8 / 2013$ & $\begin{array}{l}\text { Rinsate of decontaminated flow line after } \\
\text { collection of sample EVSB62-W-29831. }\end{array}$ \\
\hline
\end{tabular}


TABLE A.1 (Cont.)

\begin{tabular}{|c|c|c|c|c|c|c|c|c|c|}
\hline \multicolumn{2}{|c|}{$\begin{array}{c}\text { Sample } \\
\text { Date and Time }\end{array}$} & \multirow{2}{*}{$\frac{\text { Sample }}{\text { EVQCFB-W-29843 }^{\mathrm{d}}}$} & \multirow{2}{*}{$\begin{array}{l}\text { Location } \\
\text { QC }\end{array}$} & \multirow{2}{*}{$\begin{array}{c}\begin{array}{c}\text { Sample } \\
\text { Type }^{a}\end{array} \\
\text { FB }\end{array}$} & \multirow{2}{*}{$\begin{array}{c}\text { Sample } \\
\text { Matrix }^{b}\end{array}$} & \multirow{2}{*}{$\begin{array}{c}\text { Depth or } \\
\text { Height } \\
{\text { (ft BGL or } \mathrm{ft})^{\mathrm{c}}}\end{array}$} & \multirow{2}{*}{$\begin{array}{c}\text { Chain of } \\
\text { Custody } \\
6748\end{array}$} & \multirow{2}{*}{$\begin{array}{l}\begin{array}{l}\text { Shipping } \\
\text { Date }\end{array} \\
5 / 8 / 2013\end{array}$} & \multirow[b]{2}{*}{$\begin{array}{l}\text { Trip blank sent to the AGEM Laboratory for } \\
\text { organic analysis with water samples listed on } \\
\text { COC } 6748 \text {. }\end{array}$} \\
\hline $5 / 8 / 13$ & $18: 30$ & & & & & & & & \\
\hline $5 / 8 / 13$ & 18:38 & EVMW4-W-29826 & MW4 & $\mathrm{N}$ & WG & $48.5-68.5$ & 6748 & $5 / 8 / 2013$ & $\begin{array}{l}\text { Depth to water }=47.05 \mathrm{ft} \text {. Depth of } 2 \text {-in. well }= \\
68.5 \mathrm{ft} \text {. Sample collected by using a low-flow } \\
\text { bladder pump positioned at } 58.5 \mathrm{ft} \text { after } \\
\text { purging of } 7 \mathrm{~L} \text {. }\end{array}$ \\
\hline $5 / 8 / 13$ & $18: 38$ & EVMW4-W-29826VER $^{\mathrm{d}}$ & MW4 & VER & WG & $48.5-68.5$ & 6889 & $5 / 9 / 2013$ & Verification sample sent to Test America. \\
\hline $5 / 8 / 13$ & $18: 46$ & EVSB63-W-29832 & SB63 & $\mathrm{N}$ & WG & $20--25$ & 6748 & $5 / 8 / 2013$ & $\begin{array}{l}\text { Depth to water }=18.19 \mathrm{ft} \text {. Depth of } 1 \text {-in. well }= \\
25 \mathrm{ft} \text {. Sample collected by using a low-flow } \\
\text { bladder pump positioned at } 22.5 \mathrm{ft} \text { after } \\
\text { purging of } 2 \mathrm{~L} \text {. }\end{array}$ \\
\hline $5 / 8 / 13$ & $18: 46$ & EVSB63-W-29832VER ${ }^{d}$ & SB63 & VER & WG & $20-25$ & 6889 & $5 / 9 / 2013$ & Verification sample sent to Test America. \\
\hline $5 / 9 / 13$ & 9:06 & EVSB80-W-29835 & SB80 & $\mathrm{N}$ & WG & $46.2-70.7$ & 6890 & $5 / 9 / 2013$ & $\begin{array}{l}\text { Depth to water }=50.12 \mathrm{ft} \text {. Depth of } 1 \text {-in. well = } \\
70.7 \mathrm{ft} \text {. Sample collected by using a low-flow } \\
\text { bladder pump positioned at } 37.5 \mathrm{ft} \text { after } \\
\text { purging of } 2 \mathrm{~L} \text {. }\end{array}$ \\
\hline $5 / 9 / 13$ & 9:06 & EVSB80-W-29835VER ${ }^{d}$ & SB80 & VER & WG & $46.2-70.7$ & 6889 & $5 / 9 / 2013$ & Verification sample sent to Test America. \\
\hline $5 / 9 / 13$ & $10: 12$ & EVSB49-W-29829 & SB49 & $\mathrm{N}$ & WG & 51-55 & 6890 & $5 / 9 / 2013$ & $\begin{array}{l}\text { Depth to water }=41.90 \mathrm{ft} \text {. Depth of } 1 \text {-in. well }= \\
55 \mathrm{ft} \text {. Sample collected by using a low-flow } \\
\text { bladder pump positioned at } 53 \mathrm{ft} \text { after purging } \\
\text { of } 2 \mathrm{~L} \text {. }\end{array}$ \\
\hline $5 / 9 / 13$ & $10: 17$ & EVMW2-W-29824DUP ${ }^{d}$ & MW2 & DUP-L & WG & $59-79$ & 6890 & $5 / 9 / 2013$ & Duplicate laboratory analysis. \\
\hline $5 / 9 / 13$ & $10: 17$ & EVMW2-W-29824 & MW2 & $\mathrm{N}$ & WG & $59-79$ & 6890 & $5 / 9 / 2013$ & $\begin{array}{l}\text { Depth to water }=54.74 \mathrm{ft} \text {. Depth of } 4 \text {-in. well }= \\
79 \mathrm{ft} . \text { Sample collected by using a low-flow } \\
\text { bladder pump positioned at } 69 \mathrm{ft} \text { after purging } \\
\text { of } 5.5 \mathrm{~L} \text {. }\end{array}$ \\
\hline $5 / 9 / 13$ & $11: 12$ & EVSB78-W-29834 & SB78 & $\mathrm{N}$ & WG & $30-40$ & 6890 & $5 / 9 / 2013$ & $\begin{array}{l}\text { Depth to water }=26.78 \mathrm{ft} \text {. Depth of } 1 \text {-in. well }= \\
40 \mathrm{ft} \text {. Sample collected by using a low-flow } \\
\text { bladder pump positioned at } 35 \mathrm{ft} \text { after purging } \\
\text { of } 1.8 \mathrm{~L} \text {. }\end{array}$ \\
\hline $5 / 9 / 13$ & $12: 03$ & EVSB60DUP-W-29838 ${ }^{d}$ & SB60 & DUP-F & WG & $56.7-61.7$ & 6890 & $5 / 9 / 2013$ & Field replicate. \\
\hline $5 / 9 / 13$ & $12: 03$ & EVSB60-W-29830 & SB60 & $\mathrm{N}$ & WG & $56.7-61.7$ & 6890 & $5 / 9 / 2013$ & $\begin{array}{l}\text { Depth to water }=53.19 \mathrm{ft} \text {. Depth of } 1 \text {-in. well }= \\
61.7 \mathrm{ft} \text {. Sample collected by using a low-flow } \\
\text { bladder pump positioned at } 59.2 \mathrm{ft} \text { after } \\
\text { purging of } 2.5 \mathrm{~L} \text {. }\end{array}$ \\
\hline $5 / 9 / 13$ & $13: 24$ & EVSB88-W-29836 & SB88 & $\mathrm{N}$ & WG & $62-72$ & 6890 & $5 / 9 / 2013$ & $\begin{array}{l}\text { Depth to water }=55.34 \mathrm{ft} \text {. Depth of } 1 \text {-in. well }= \\
72 \mathrm{ft} . \text { Sample collected by using a low-flow } \\
\text { bladder pump positioned at } 67 \mathrm{ft} \text { after purging } \\
\text { of } 2 \mathrm{~L} \text {. }\end{array}$ \\
\hline
\end{tabular}




\begin{tabular}{|c|c|c|c|c|c|c|c|c|c|}
\hline $\begin{array}{l}\text { Sampl } \\
\text { Date and }\end{array}$ & $\begin{array}{l}\text { e } \\
\text { Time }\end{array}$ & Sample & Location & $\begin{array}{l}\text { Sample } \\
\text { Type }^{\mathrm{a}}\end{array}$ & $\begin{array}{l}\text { Sample } \\
\text { Matrix }^{\mathbf{b}}\end{array}$ & $\begin{array}{c}\text { Depth or } \\
\text { Height } \\
\text { (ft BGL or } \mathrm{ft})^{\mathrm{c}}\end{array}$ & $\begin{array}{l}\text { Chain of } \\
\text { Custody }\end{array}$ & $\begin{array}{l}\text { Shipping } \\
\text { Date }\end{array}$ & Sample Description \\
\hline $5 / 9 / 13$ & $13: 30$ & EVSB09-W-29827 & SB09 & $\mathrm{N}$ & WG & $51-57$ & 6890 & $5 / 9 / 2013$ & $\begin{array}{l}\text { Depth to water }=27.78 \mathrm{ft} \text {. Depth of } 1 \text {-in. well = } \\
57 \mathrm{ft} \text {. Sample collected by using a low-flow } \\
\text { bladder pump positioned at } 54 \mathrm{ft} \text { after purging } \\
\text { of } 1.8 \mathrm{~L} \text {. }\end{array}$ \\
\hline 9/24/13 & 13:08 & EV027-B-29792 & EV027 & $\mathrm{N}$ & VB & 4 & 924130 & 9/24/2013 & Hackberry. First-degree branch, $4 \mathrm{ft}$ up, on north. \\
\hline $9 / 24 / 13$ & 13:14 & EV028-B-29793 & EV028 & $\mathrm{N}$ & VB & 3 & 924130 & $9 / 24 / 2013$ & $\begin{array}{l}\text { Willow on north side of creek. Second-degree } \\
\text { branch, } 3 \mathrm{ft} \text { up, on south. }\end{array}$ \\
\hline 9/24/13 & $13: 18$ & EV004-B-29795 & EV004 & $\mathrm{N}$ & VB & 3 & 924130 & 9/24/2013 & Elm. Second-degree branch, $3 \mathrm{ft}$ up, on south. \\
\hline $9 / 24 / 13$ & 13:18 & EV013A-B-29794 & EV013A & $\mathrm{N}$ & VB & 2 & 924130 & $9 / 24 / 2013$ & $\begin{array}{l}\text { Honey locust. First-degree branch, } 2 \text { feet up, on } \\
\text { south. }\end{array}$ \\
\hline 9/24/13 & $13: 18$ & EV016-B-29797 & EV016 & $\mathrm{N}$ & VB & 4 & 924130 & 9/24/2013 & Willow. Second-degree branch, $4 \mathrm{ft}$ up, on south. \\
\hline 9/24/13 & $13: 28$ & EV021-B-29796 & EV021 & $\mathrm{N}$ & VB & 4 & 924130 & 9/24/2013 & Willow. First-degree branch, $4 \mathrm{ft}$ up, on south. \\
\hline 9/24/13 & $13: 48$ & EV022-B-29798 & EV022 & $\mathrm{N}$ & VB & 4 & 924130 & $9 / 24 / 2013$ & $\begin{array}{l}\text { Osage orange. First-degree branch, } 4 \mathrm{ft} \text { up, on } \\
\text { south. }\end{array}$ \\
\hline 9/24/13 & $13: 48$ & EV022-B-29798DUP ${ }^{d}$ & EV022 & DUP-L & VB & 4 & 924130 & 9/24/2013 & Duplicate laboratory analysis. \\
\hline 9/24/13 & $14: 08$ & EV024-B-29799 & EV024 & $\mathrm{N}$ & VB & 6 & 924130 & 9/24/2013 & Elm. First-degree branch, $6 \mathrm{ft}$ up, on southwest. \\
\hline $9 / 24 / 13$ & $14: 12$ & EV017-B-29800 & EV017 & $\mathrm{N}$ & VB & 1 & 924130 & $9 / 24 / 2013$ & $\begin{array}{l}\text { Mulberry, flagging. First-degree branch, } 1 \mathrm{ft} \text { up, } \\
\text { on south. }\end{array}$ \\
\hline $9 / 24 / 13$ & $14: 20$ & EV018-B-29801 & EV018 & $\mathrm{N}$ & VB & 2 & 924130 & $9 / 24 / 2013$ & $\begin{array}{l}\text { Mulberry, stake. First-degree branch, } 2 \mathrm{ft} \text { up, on } \\
\text { south. }\end{array}$ \\
\hline $9 / 24 / 13$ & $14: 26$ & EV006-B-29802 & EV006 & $\mathrm{N}$ & VB & 3 & 924130 & $9 / 24 / 2013$ & $\begin{array}{l}\text { Cherry tree. First-degree branch, } 3 \mathrm{ft} \text { up, on } \\
\text { southwest. }\end{array}$ \\
\hline 9/24/13 & $14: 30$ & EV019-B-29803 & EV019 & $\mathrm{N}$ & VB & 4 & 924130 & 9/24/2013 & Second-degree branch, $4 \mathrm{ft}$ up, on east. \\
\hline $9 / 24 / 13$ & $14: 35$ & EV020-B-29804 & EV020 & $\mathrm{N}$ & VB & 6 & 924130 & $9 / 24 / 2013$ & $\begin{array}{l}\text { Mulberry. Second-degree branch, } 6 \mathrm{ft} \text { up, on } \\
\text { southeast. }\end{array}$ \\
\hline 9/24/13 & $14: 35$ & EV020-B-29804DUP ${ }^{d}$ & EV020 & DUP-L & VB & 6 & 924130 & $9 / 24 / 2013$ & Duplicate laboratory analysis. \\
\hline 9/24/13 & $14: 42$ & EV008A-B-29805 & EV008A & $\mathrm{N}$ & VB & 3 & 924130 & 9/24/2013 & Hackberry. First-degree branch, $3 \mathrm{ft}$ up, on south. \\
\hline 9/24/13 & $14: 42$ & EV008A-B-29805DUP $^{d}$ & EV008A & DUP-L & VB & 3 & 924130 & 9/24/2013 & Duplicate laboratory analysis. \\
\hline $9 / 24 / 13$ & & - & - & - & - & - & - & - & $\begin{array}{l}\text { Tree gone. Removed to make way for agricultural } \\
\text { field. }\end{array}$ \\
\hline $9 / 24 / 13$ & $14: 58$ & EV026-B-29806 & EV026 & $\mathrm{N}$ & VB & 6 & 924130 & $9 / 24 / 2013$ & $\begin{array}{l}\text { Black walnut. Second-degree branch, } 6 \mathrm{ft} \text { up, on } \\
\text { south. }\end{array}$ \\
\hline $9 / 24 / 13$ & $15: 00$ & EV010-B-29807 & EV010 & $\mathrm{N}$ & VB & 3 & 924131 & $9 / 24 / 2013$ & $\begin{array}{l}\text { Willow in creek (dry). First-degree branch, } 3 \mathrm{ft} \text { up, } \\
\text { on south. }\end{array}$ \\
\hline $9 / 24 / 13$ & $15: 05$ & EV009-B-29808 & EV009 & $\mathrm{N}$ & VB & 4 & 924131 & $9 / 24 / 2013$ & $\begin{array}{l}\text { Willow in creek, north of } 010 \text {. Second-degree } \\
\text { branch, } 4 \mathrm{ft} \text { up, on northwest. }\end{array}$ \\
\hline $9 / 24 / 13$ & $15: 18$ & EV029-B-29809 & EV029 & $\mathrm{N}$ & VB & 8 & 924131 & $9 / 24 / 2013$ & $\begin{array}{l}\text { Ash by driveway, } 1191 \text { Prairie Road. First-degree } \\
\text { branch, } 8 \mathrm{ft} \text { up, on southeast. }\end{array}$ \\
\hline
\end{tabular}


a Sample types: DUP-F, field replicate; DUP-L, duplicate laboratory analysis; FB, field blank; N, primary sample; RI, rinsate; TB, trip blank; VER, verification sample.

b Matrix codes: VB, vegetation-branch; WG, groundwater; WQC, QA/QC water sample (e.g., trip blank); WS, surface water.

c For groundwater samples, sample depth ( $\mathrm{ft} \mathrm{BGL}$ ) is given. For vegetation samples, height above the ground (ft) is given.

d Quality control sample. 


\section{Appendix B:}

\section{Quality Control Data Summary}


TABLE B.1 Analytical results from the AGEM Laboratory for quality control samples collected during annual monitoring in 2013.

\begin{tabular}{|c|c|c|c|c|c|c|c|}
\hline \multirow[b]{2}{*}{ Location } & \multirow[b]{2}{*}{ Sample } & \multirow[b]{2}{*}{$\begin{array}{l}\text { Sample } \\
\text { Date }\end{array}$} & \multirow[b]{2}{*}{$\begin{array}{l}\text { Sample } \\
\text { Type }^{a}\end{array}$} & \multirow[b]{2}{*}{$\begin{array}{c}\text { Depth or } \\
\text { Height } \\
\text { (ft BGL or } \mathrm{ft})^{\mathrm{b}}\end{array}$} & \multicolumn{3}{|c|}{$\begin{array}{c}\text { Concentration } \\
(\mu \mathrm{g} / \mathrm{L} \text { in groundwater; } \mu \mathrm{g} / \mathrm{kg} \text { in vegetation })\end{array}$} \\
\hline & & & & & $\begin{array}{l}\text { Carbon } \\
\text { Tetrachloride }\end{array}$ & Chloroform & $\begin{array}{l}\text { Methylene } \\
\text { Chloride }\end{array}$ \\
\hline EV005 & EV005-W-29820 & $5 / 8 / 13$ & $\mathrm{~N}$ & - & $N^{c}$ & ND & ND \\
\hline EV005 & EV005-W-29820DUP & $5 / 8 / 13$ & DUP-L & - & ND & ND & ND \\
\hline MW1 & EVMW1-W-29823 & $5 / 8 / 13$ & $\mathrm{~N}$ & $41-51$ & 3.1 & ND & ND \\
\hline MW1 & EVMW1-W-29823DUP & $5 / 8 / 13$ & DUP-L & $41-51$ & 2.9 & ND & ND \\
\hline MW2 & EVMW2-W-29824 & $5 / 9 / 13$ & $\mathrm{~N}$ & $59-79$ & 87 & 2.7 & ND \\
\hline MW2 & EVMW2-W-29824DUP & $5 / 9 / 13$ & DUP-L & $59-79$ & 86 & 2.6 & ND \\
\hline MW3 & EVMW3-W-29825 & $5 / 8 / 13$ & $\mathrm{~N}$ & $56.5-71.5$ & 17 & ND & ND \\
\hline MW3 & EVMW3DUP-W-29837 & $5 / 8 / 13$ & DUP-F & $56.5-71.5$ & 18 & ND & ND \\
\hline MW3 & EVMW3DUP-W-29837DUP & $5 / 8 / 13$ & DUP-L & $56.5-71.5$ & 17 & ND & ND \\
\hline SB60 & EVSB60-W-29830 & $5 / 9 / 13$ & $\mathrm{~N}$ & $56.7-61.7$ & 85 & 3.1 & ND \\
\hline SB60 & EVSB60DUP-W-29838 & $5 / 9 / 13$ & DUP-F & $56.7-61.7$ & 94 & 3.4 & ND \\
\hline EV008A & EV008A-B-29805 & $9 / 24 / 13$ & $\mathrm{~N}$ & 3 & ND & ND & - \\
\hline EV008A & EV008A-B-29805DUP & $9 / 24 / 13$ & DUP-L & 3 & ND & ND & - \\
\hline EV020 & EV020-B-29804 & 9/24/13 & $\mathrm{N}$ & 6 & ND & ND & - \\
\hline EV020 & EV020-B-29804DUP & $9 / 24 / 13$ & DUP-L & 6 & ND & ND & - \\
\hline EV022 & EV022-B-29798 & $9 / 24 / 13$ & $\mathrm{~N}$ & 4 & ND & ND & - \\
\hline EV022 & EV022-B-29798DUP & $9 / 24 / 13$ & DUP-L & 4 & ND & ND & - \\
\hline QC & EVDIH2O-W-29841 & $5 / 8 / 13$ & FB & - & ND & ND & ND \\
\hline QC & EVQCFB-W-29843 & $5 / 8 / 13$ & FB & - & ND & ND & ND \\
\hline QC & EVQCIR-W-29839 & $5 / 8 / 13$ & $\mathrm{RI}$ & - & ND & ND & ND \\
\hline QC & EVQCIR-W-29840 & $5 / 8 / 13$ & $\mathrm{RI}$ & - & ND & ND & ND \\
\hline QC & EVQCTB-W-29842 & $5 / 8 / 13$ & TB & - & ND & ND & ND \\
\hline
\end{tabular}

a Sample types: DUP-F, field replicate; DUP-L, duplicate laboratory analysis; FB, field blank; N, primary sample; RI, rinsate; TB, trip blank.

b For groundwater samples, sample depth (ft BGL) is given. For vegetation samples from locations EV008A, EV020, and EV022, sample height (ft) is given.

c ND, Compound analyzed for but not detected at a level greater than or equal to the method detection limit $(<1 \mu \mathrm{g} / \mathrm{L}$ for VOCs in groundwater samples; $<0.1 \mu \mathrm{g} / \mathrm{kg}$ for carbon tetrachloride or $<0.75 \mu \mathrm{g} / \mathrm{kg}$ for chloroform in vegetation samples). 
TABLE B.2 Analytical results for verification groundwater samples collected during annual monitoring in 2013.

\begin{tabular}{|c|c|c|c|c|c|c|c|}
\hline \multirow[b]{2}{*}{ Location } & \multirow[b]{2}{*}{ Sample } & \multirow[b]{2}{*}{$\begin{array}{l}\text { Sample } \\
\text { Date and } \\
\text { Time }\end{array}$} & \multirow[b]{2}{*}{$\begin{array}{l}\text { Depth } \\
\text { from } \\
\text { (ft BGL) }\end{array}$} & \multirow[b]{2}{*}{$\begin{array}{l}\text { Analytical } \\
\text { Laboratory }\end{array}$} & \multicolumn{3}{|c|}{ Concentration $(\mu \mathrm{g} / \mathrm{L})$} \\
\hline & & & & & $\begin{array}{l}\text { Carbon } \\
\text { Tetrachloride }\end{array}$ & Chloroform & $\begin{array}{c}\text { Methylene } \\
\text { Chloride }\end{array}$ \\
\hline MW1 & EVMW1-W-29823 & $5 / 8 / 2013$ & $41-51$ & AGEM & 3.1 & $N^{a}$ & ND \\
\hline MW1 & EVMW1-W-29823VER & $5 / 8 / 2013$ & $41-51$ & TestAmerica & 3.9 & 0.6 & ND \\
\hline MW4 & EVMW4-W-29826 & $5 / 8 / 2013$ & $48.5-68.5$ & AGEM & 70 & 2.4 & ND \\
\hline MW4 & EVMW4-W-29826VER & $5 / 8 / 2013$ & $48.5-68.5$ & TestAmerica & $78 D^{b}$ & 2.8 & ND \\
\hline SB63 & EVSB63-W-29832 & $5 / 8 / 2013$ & $20-25$ & AGEM & ND & ND & ND \\
\hline SB63 & EVSB63-W-29832VER & $5 / 8 / 2013$ & $20-25$ & TestAmerica & ND & ND & ND \\
\hline SB80 & EVSB80-W-29835 & $5 / 9 / 2013$ & $46.2-70.7$ & AGEM & 2.1 & ND & ND \\
\hline SB80 & EVSB80-W-29835VER & $5 / 9 / 2013$ & $46.2-70.7$ & TestAmerica & 2.1 & $0.1 \mathrm{Jc}^{\mathrm{c}}$ & ND \\
\hline QC & EVQCTB-W-29842 & $5 / 8 / 2013$ & - & AGEM & ND & ND & ND \\
\hline QC & EVQCTB-W-29842VER & $5 / 8 / 2013$ & - & TestAmerica & ND & ND & ND \\
\hline
\end{tabular}

a ND, compound analyzed for but not detected at a level greater than or equal to the method detection limit $(<1 \mu \mathrm{g} / \mathrm{L}$ for analyses at the AGEM Laboratory; $<0.5 \mu \mathrm{g} / \mathrm{L}$ for analyses at TestAmerica).

b D, results obtained from analysis at a secondary dilution factor.

c J, compound identified with an estimated concentration between the instrument detection limit and the method detection limit. 
Supplement 1:

Groundwater Verification Data from TestAmerica Laboratories, Inc. 


\section{TestAmerica}

THE LEADER IN ENVIRONMENTAL TESTING

\section{ANALYTICAL REPORT}

Job Number: 200-16407-1

SDG Number: 16407-1

Job Description: Everest (200-16407)

Contract Number: 1E-30401

For:

Argonne National Laboratory

9700 South Cass Avenue

Building 203

Office B-141

Argonne, IL 60439

Attention: Ms. Esther Bowen

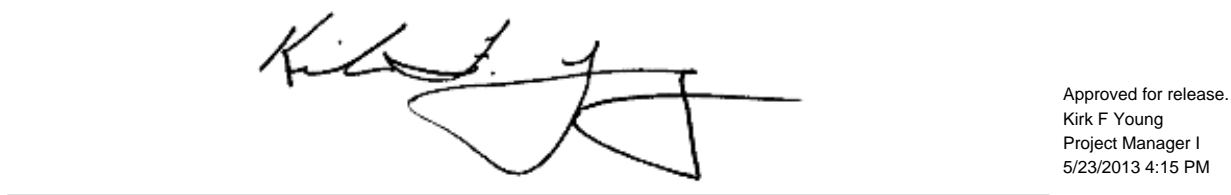

Kirk F Young, Project Manager I

30 Community Drive, South Burlington, VT, 05403

(802)660-1990

kirk.young@testamericainc.com

$05 / 23 / 2013$

The test results in this report relate only to sample(s) as received by the laboratory. These test results were derived under a quality system that adheres to the requirements of NELAC. Pursuant to NELAC, this report may not be produced in full without written approval from the laboratory 


\section{Table of Contents}

Cover Title Page . . . . . . . . . . . . . . . . 1

Report Narrative . . . . . . . . . . . . . . . 4

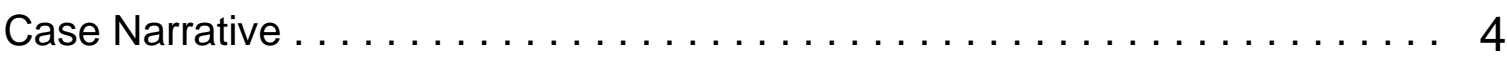

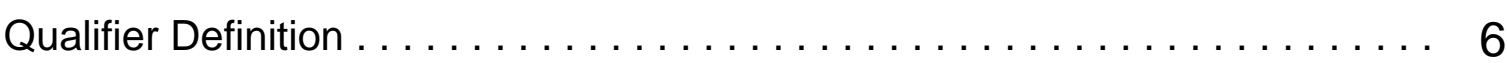

External Chain of Custody . . . . . . . . . . . . . . 7

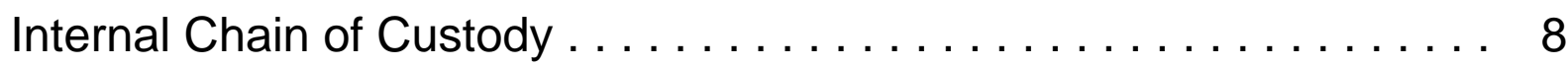

Shipping Documentation . . . . . . . . . . . . . . 9

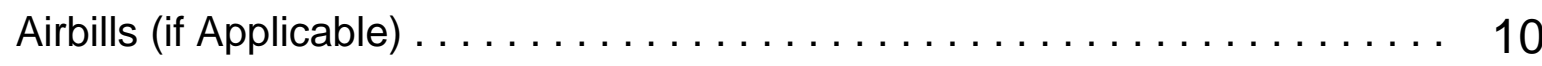

Sample Receipt and Log In Check List .................. 11

Methodology Review . . . . . . . . . . . . . . . . . . . 13

QC Summary - SOM01.2 Volatiles-Trace . . . . . . . . . . . . . . . 14

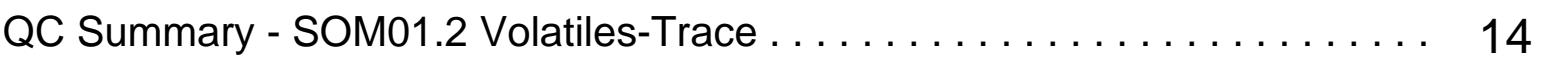

Deuterated Monitoring Compound Summary . . . . . . . . . . . . . . . 14

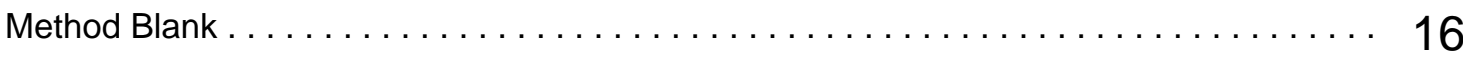

GC/MS Instrument Performance Check $\ldots \ldots \ldots \ldots \ldots \ldots \ldots \ldots \ldots \ldots \ldots \ldots \ldots \ldots$

Internal Standard Area and RT Summary $\ldots \ldots \ldots \ldots \ldots \ldots \ldots \ldots \ldots \ldots \ldots \ldots \ldots$

Sample Data - SOM01.2 Volatiles-Trace . . . . . . . . . . . 20

Sample Data - SOM01.2 Volatiles-Trace . . . . . . . . . . . . . 20

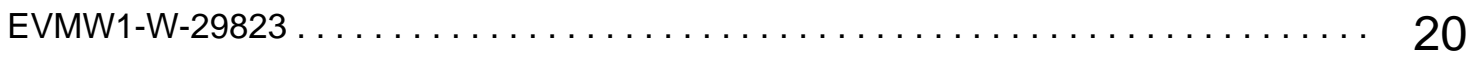

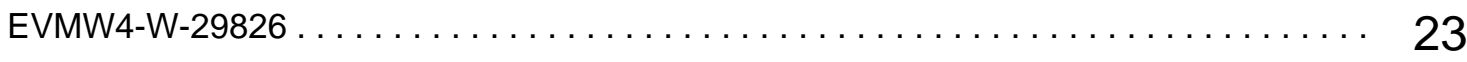

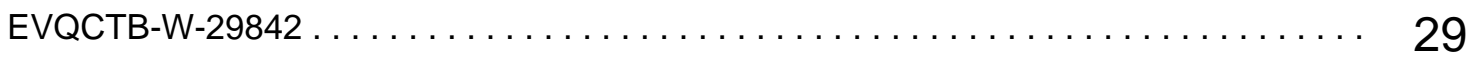

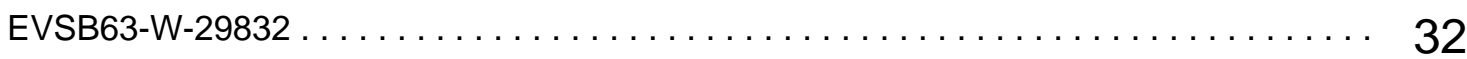

EVSB80-W-29835 ........................................ 35

Standards - SOM01.2 Volatiles-Trace . . . . . . . . . . . . . 38

Standards - SOM01.2 Volatiles-Trace . . . . . . . . . . . . . . 38

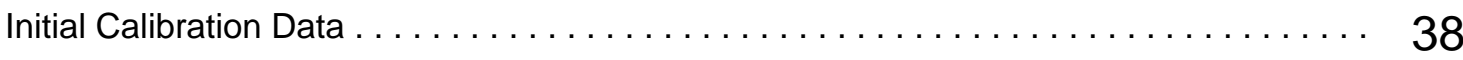

Page 2 of $62 \quad 05 / 23 / 2013$ 


\section{Table of Contents}

CCV Data, including closing CCV $\ldots \ldots \ldots \ldots \ldots \ldots \ldots \ldots \ldots \ldots \ldots \ldots \ldots \ldots \ldots \ldots \ldots$

Raw Qc Data - SOM01.2 Volatiles-Trace . . . . . . . . . . . . 47

Raw Qc Data - SOM01.2 Volatiles-Trace .................... 47

Raw Qc Data - SOM01.2 Volatiles-Trace . . . . . . . . . . . . . . . 47

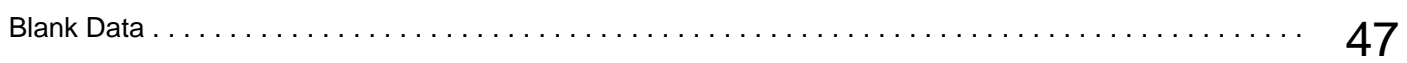

Standards Traceability $\ldots \ldots \ldots \ldots \ldots \ldots \ldots \ldots \ldots \ldots \ldots \ldots \ldots$ 
CASE NARRATIVE

\title{
Client: Argonne National Laboratory
}

\author{
Project: Everest (200-16407)
}

Report Number: 200-16407-1

Enclosed is the data set for the referenced project work. With the exceptions noted as flags or footnotes, standard analytical protocols were followed in performing the analytical work and the applied control limits were met.

Calculations were performed before rounding to avoid round-off errors in calculated results.

All holding times were met and proper preservation noted for the methods performed on these samples, unless otherwise detailed in the individual sections below.

\section{Receipt}

The samples were received on 05/10/2013. Documentation of the condition of the samples at the time of their receipt and any exception to the laboratory's Sample Acceptance Policy is documented in the Shipping Documentation section of this submittal. The samples, as received, were not acid preserved. On that basis, the laboratory did provide for the analysis of the samples within seven days of sample collection.

\section{SOM01.2 Volatile Organics (Trace Level Water)}

A storage blank was prepared for volatile organics analysis, and stored in association with the storage of the samples. That storage blank, identified as VHBLK01, was carried through the holding period with the samples, and analyzed.

Sample EVMW4-W-29826 was analyzed at a dilution, based on the results of preliminary screening. An additional, more concentrated analysis was performed on sample EVMW4-W-29826 in order to provide for a lower reporting limit for those analytes that were not identified in the primary analysis. Both sets of results for the analysis of sample EVMW4-W-29826 are included in this submittal.

Each of the analyses associated with the sample set exhibited an acceptable internal standard performance. There was an acceptable recovery of each deuterated monitoring compound (DMC) in the analysis of the method blank associated with the analytical work, and in the analysis of the storage blank associated with the sample set. The analysis of the samples in this sample set did meet the technical acceptance criteria specific to DMC recoveries, although not all DMC recoveries were within the control range in each analysis. The technical acceptance criteria does provide for the recovery of up to three DMCs to fall outside of the control range in the analysis of field samples. Matrix spike and matrix spike duplicate analyses were not performed on samples in this sample set. Trace concentrations of bromomethane and methylene chloride were identified in the analysis of the method blank associated with the analytical work. The concentration of each target analyte in that analysis was below the established reporting limit, and the analysis did meet the technical acceptance criteria for a compliant method blank analysis. The analysis of the storage blank associated with the sample set was free of analyte contamination. Trace concentrations of methylene chloride and carbon tetrachloride were identified in the analysis of the instrument blank associated with the analytical work. The 
concentration of each target analyte in that analysis was below the established reporting limit, and the analysis did meet the technical acceptance criteria for a compliant instrument blank analysis. Present in the method blank, storage blank, and instrument blank analyses was a nontarget constituent that represents a compound that is related to the DMC formulation. The fact that the presence of this compound is not within the laboratory's control is at issue. The derived results for that compound have been qualified with an "X" qualifier to reflect the source of the contamination.

The responses for each of the target analytes met the relative standard deviation criterion in the initial calibration. The response for each target analyte met the percent difference criterion in the opening/continuing calibration check acquisition. The response for each target analyte met the 50.0 percent difference criterion in the closing calibration check acquisition.

The primary quantitation mass for methylcyclohexane that is specified in the Statement of Work is mass 83 . The laboratory did identify a contribution to mass 83 from 1,2-dichloropropane- $\mathrm{d}_{6}$, one of the deuterated monitoring compounds (DMCs). The laboratory did change the primary quantitation mass assignment to mass 55 for the quantification of methylcyclohexane.

Manual integration was employed in deriving certain of the analytical results. The values that have been derived from manual integration are qualified on the quantitation reports. Extracted ion current profiles for each manual integration are included in the data package, and further documented at the end of this submittal. 


\section{DATA REPORTING QUALIFIERS}

Client: Argonne National Laboratory

Job Number: $200-16407-1$

Sdg Number: 16407-1

GC/MS VOA

U

E

$J$

$J$

D

$X$

B
Analyzed for but not detected.

Compound concentration exceeds the upper level of the calibration range of the instrument for that specific analysis.

Indicates an Estimated Value for TICs

Indicates an estimated value.

Sample was analyzed at a higher dilution factor.

See case narrative notes for explanation of the ' $X$ ' flag

Surrogate exceeds the control limit

The analyte was found in an associated blank, as well as in the sample. 


\section{Argonne}

\section{$\therefore 89$}

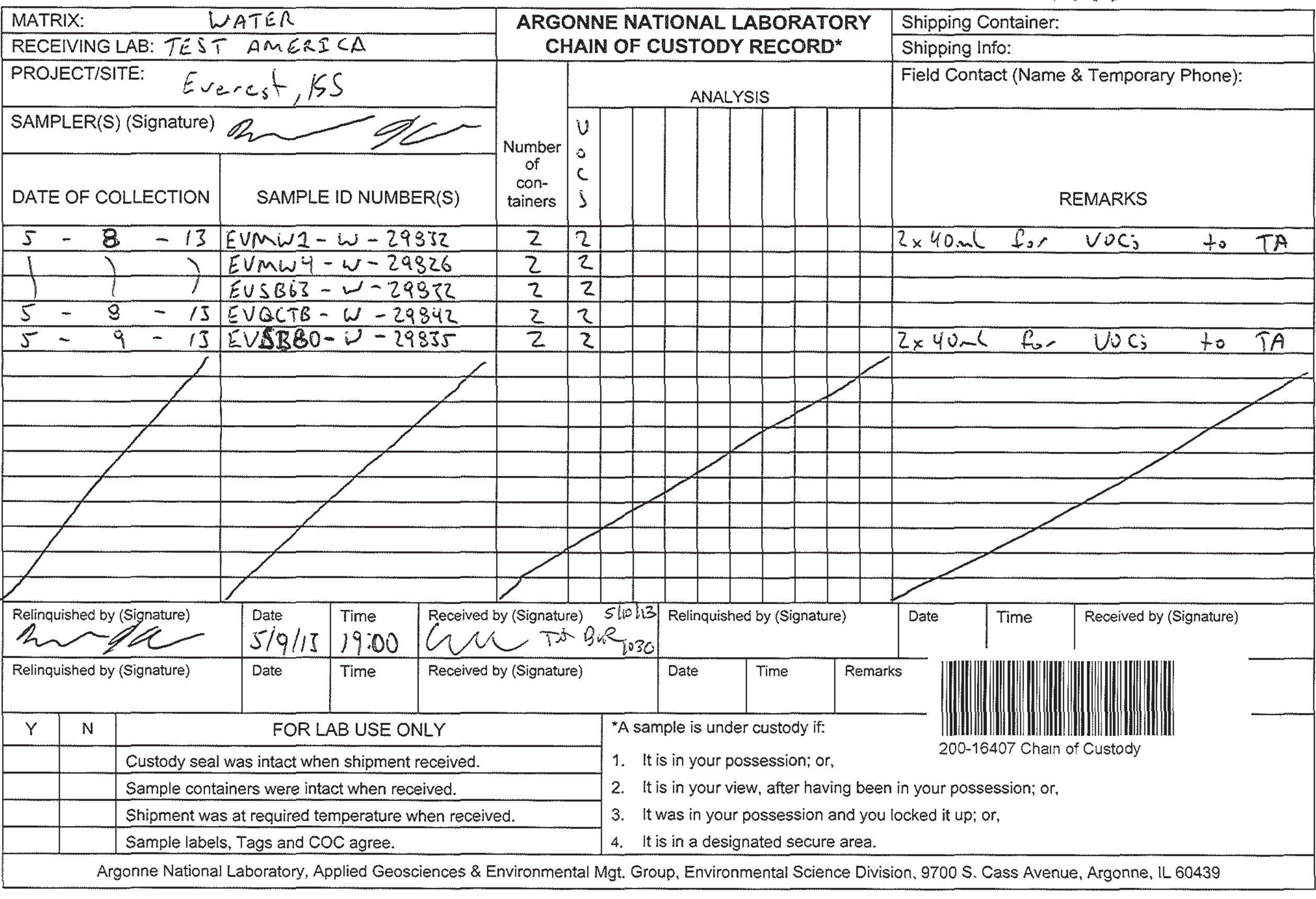




\section{Shipping and Receiving Documents}




\section{Fed Ins NEW Package Express US Airbill}

1 From

Date $5=$ 3 Sender's
Name Company

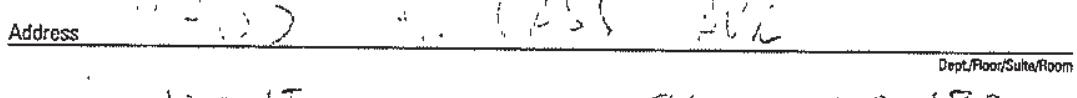

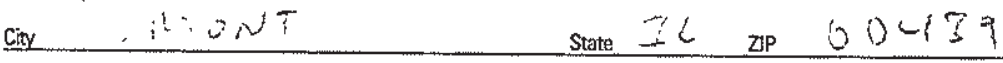

2 Your Internal Billing Reference $\quad 3,2,3-1: 3$

3 To

Recipient's $6 . \forall ; \therefore$. Phope $3 \times 360.990$

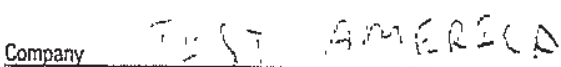

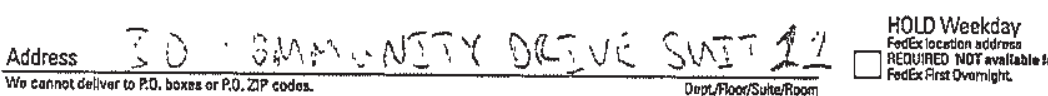
HOL Saturday Address

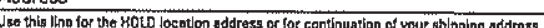

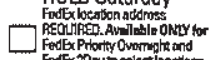

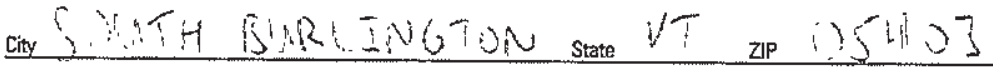

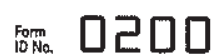

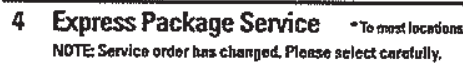

Packages up to 150 los

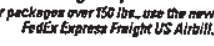

Next Business 20

$\square$ FedEx First Qvernight

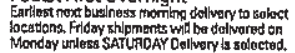

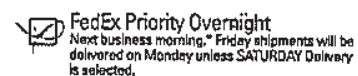
$\square$ FedEx Standard Overnight

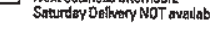

\section{Packaging ${ }^{\circ}$ Declaved value Illitison}

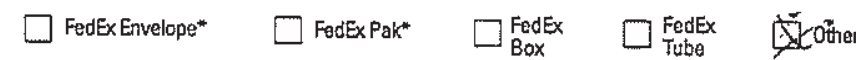

\section{Special Handling and Delivery Signature 0ptions}

wa

$\square$. FedEx 2Day A.M.

$\square$ FedEx 20ay

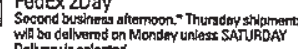

$\square$ FedEx Express Saver

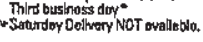

$\square$ SATURDAY Delivery,

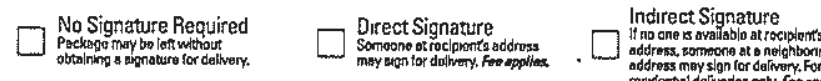
Doos this shipment contain dangerous goods?

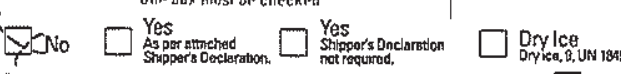

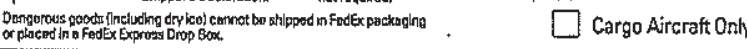

7 Payment Bill io

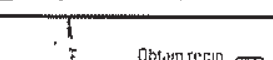

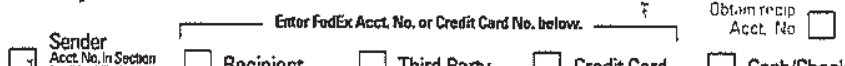

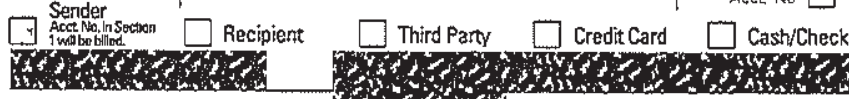
Tomal Packages ToralWeight Tow Packages Toal weight 1 crovincordiner

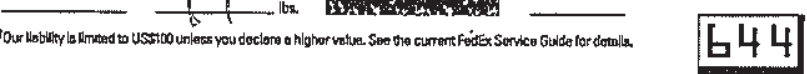

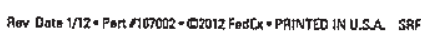

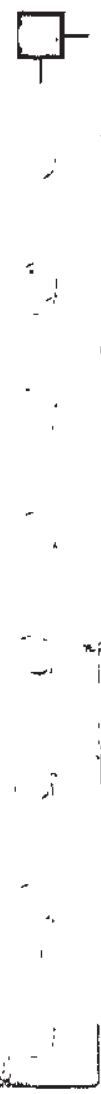




\section{Login Sample Receipt Checklist}

Client: Argonne National Laboratory

Job Number: 200-16407-1

SDG Number: 16407-1

Login Number: 16407

List Source: TestAmerica Burlington

List Number: 1

Creator: Marion, Greg T

Question

Radioactivity wasn't checked or is $</=$ background as measured by a survey meter.

The cooler's custody seal, if present, is intact.

Sample custody seals, if present, are intact.

The cooler or samples do not appear to have been compromised or tampered with

Samples were received on ice.

Cooler Temperature is acceptable.

Cooler Temperature is recorded.

$\mathrm{COC}$ is present.

COC is filled out in ink and legible.

$\mathrm{COC}$ is filled out with all pertinent information.

Is the Field Sampler's name present on COC?

There are no discrepancies between the containers received and the COC.

Samples are received within Holding Time.

Sample containers have legible labels.

Containers are not broken or leaking.

Sample collection date/times are provided.

Appropriate sample containers are used.

Sample bottles are completely filled.

Sample Preservation Verified.

There is sufficient vol. for all requested analyses, incl. any requested MS/MSDs

Containers requiring zero headspace have no headspace or bubble is $<6 \mathrm{~mm}(1 / 4 ")$.

Multiphasic samples are not present.

Answer

N/A

True

N/A

True

True

True

True

True

True

False

True

False

True

True

True

True

True

True

N/A

True

True

N/A

Samples do not require splitting or compositing.

$N / A$

Residual Chlorine Checked.

N/A
Comment

Lab does not accept radioactive samples.

NO SEAL NUMBERS

$3.0^{\circ} \mathrm{C}$ IR GUN ID $181 / \mathrm{CF}=0$

The COC does not list the sample times

EVMW1-W-29823 (200-16407-1) - Identifier logged in from sample label.

Refer to Job Narrative for details. 


\section{Sample Login Acknowledgement}

Job 200-16407-1

\begin{tabular}{|c|c|c|c|}
\hline Client Job Description: & Everest (200-16407) & Report To: & Argonne National Laboratory \\
\hline Purchase Order \#: & $1 \mathrm{E}-30401$ & & Jorge Alvarado \\
\hline Work Order \#: & $1 \mathrm{E}-30401$ & & 9700 South Cass Avenue \\
\hline Project Manager: & Kirk F Young & & Building 203 \\
\hline Job Due Date: & $5 / 24 / 2013$ & & Office B-141 \\
\hline . & 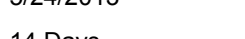 & & Argonne, IL 60439 \\
\hline Job TAT: & 14 Days & & \\
\hline \multirow[t]{2}{*}{ Max Deliverable Level: } & IV & Bill To: & Argonne National Laboratory \\
\hline & & & Accounts Payable \\
\hline \multirow[t]{4}{*}{ Earliest Deliverable Due: } & $5 / 24 / 2013$ & & Chief Financial Offices \\
\hline & & & 9700 S. Cass Ave. \\
\hline & & & Building 201 \\
\hline & & & Argonne, IL 60439 \\
\hline
\end{tabular}

\section{Login 200-16407}

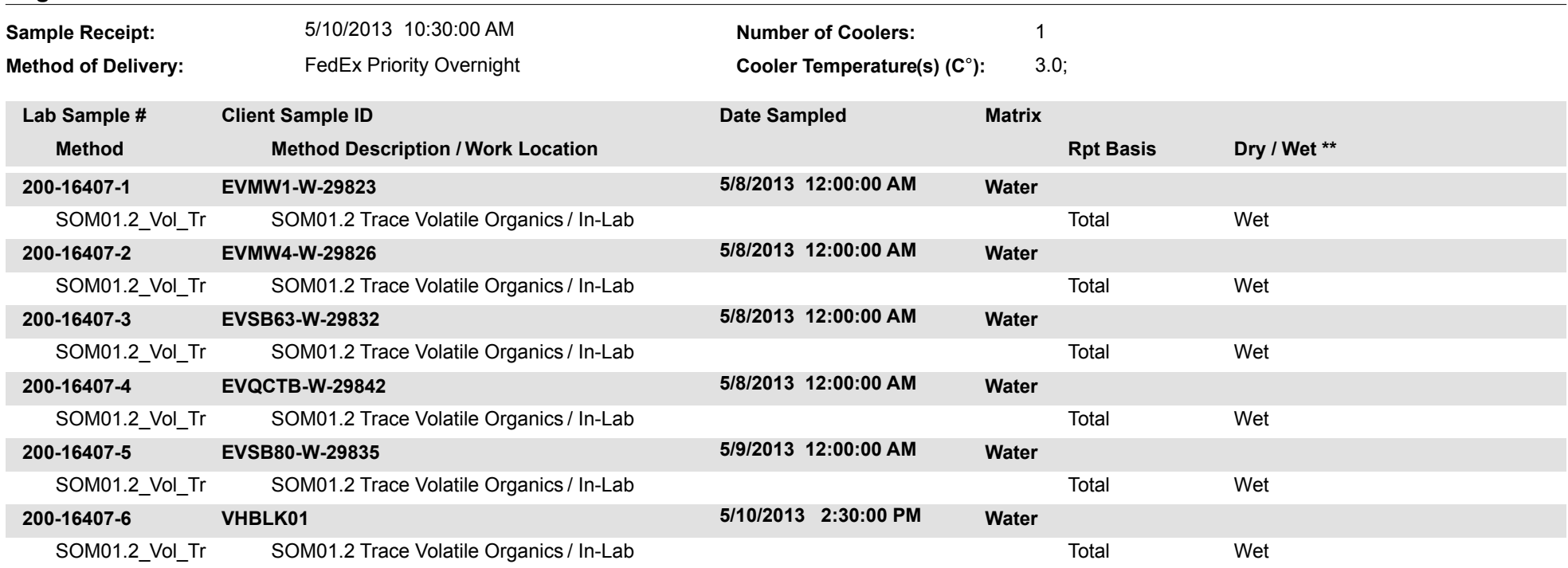




\section{METHODOLOGY SUMMARY}

$\begin{array}{llll}\text { Laboratory: } & \text { TestAmerica Laboratories } & \text { Project No: } \\ \text { Location: } & \text { South Burlington, Vermont } & \text { SDG No: } & 16407-1\end{array}$

VOA

Volatile Organics Trace - USEPA CLP SOM01.2 


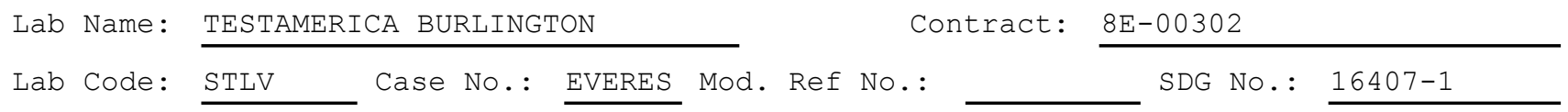
Level: (TRACE or LOW) TRACE

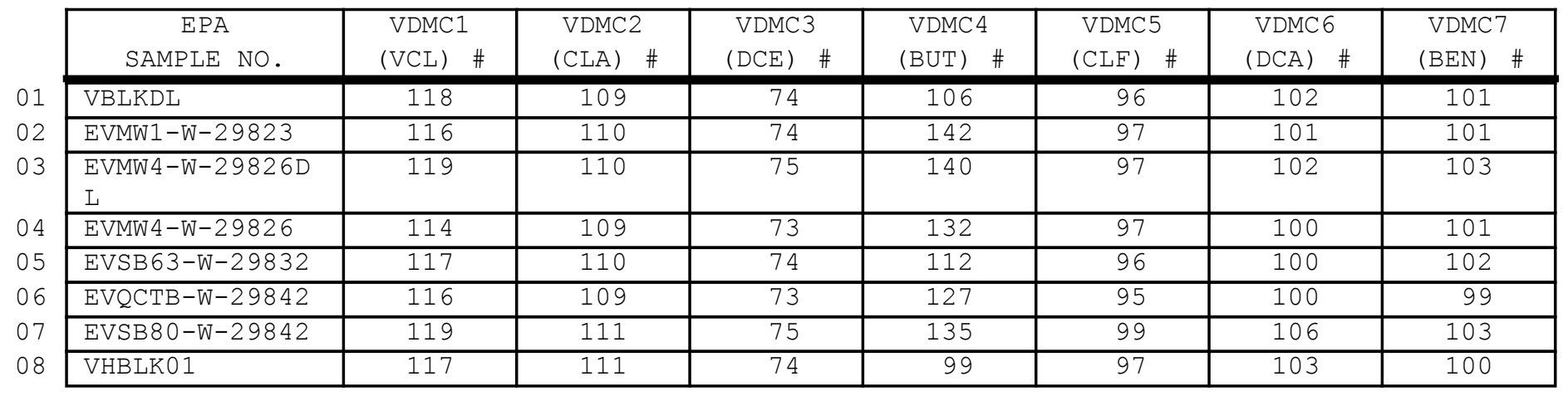

\footnotetext{
VDMC1 (VCL) = Vinyl Chloride-d3

VDMC2 (CLA) = Chloroethane-d5

VDMC3 $(\mathrm{DCE})=1,1-\mathrm{Dichloroethene-d2}$

VDMC4 $(B U T)=2-$ Butanone-d5

VDMC5 $($ CLF $)=$ Chloroform-d

VDMC6 $(\mathrm{DCA})=1,2-\mathrm{Dich}$ loroethane-d4

$\operatorname{VDMC7}$
}

$$
\begin{gathered}
\frac{\mathrm{QC} \text { LIMITS }}{(65-131)} \\
(71-131) \\
(55-104) \\
(49-155) \\
(78-121) \\
(78-129) \\
(77-124)
\end{gathered}
$$

\# Column to be used to flag recovery values

* Values outside of contract required QC limits 
2B - FORM II VOA-2

WATER VOLATILE DEUTERATED MONITORING COMPOUND RECOVERY

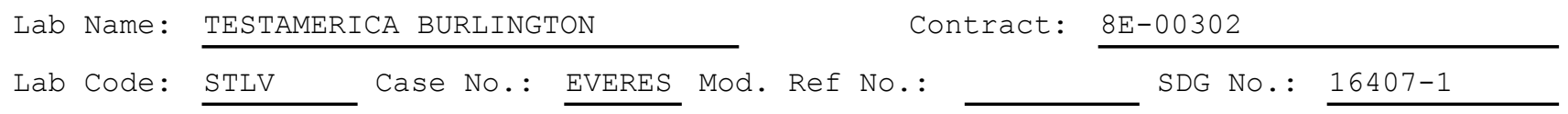

Level: (TRACE or LOW) TRACE

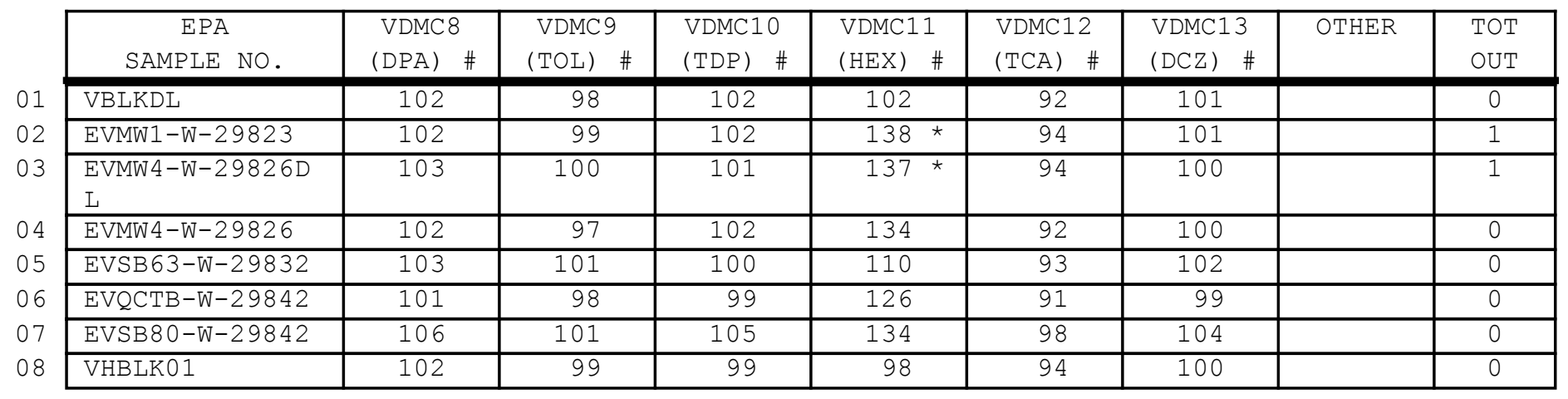

VDMC8 $\quad(\mathrm{DPA})=1,2-\mathrm{Dich}$ loropropane-d6

VDMC9 (TOL) = Toluene-d8

VDMC10 $(\mathrm{TDP})=$ trans-1,3-Dichloropropene-d4

VDMC11 $($ HEX) $=2$-Hexanone-d5

VDMC12 $(\mathrm{TCA})=1,1,2,2$-Tetrachloroethane-d2

VDMC13 $(\mathrm{DCZ})=1,2-\mathrm{Dich}$ lorobenzene-d4
QC LIMITS

$(79-124)$

$(77-121)$

$(73-121)$

$(28-135)$

$(73-125)$

$(80-131)$

\# Column to be used to flag recovery values

* Values outside of contract required QC limits

Report 1,4-Dioxane-d8 for Low-Medium VOA analysis only

Page $\underline{1}$ of 1 
Lab Name: TESTAMERICA BURLINGTON

Lab Code: STLV Case No.: EVERES Mod. Ref No.:

Lab File ID: DJPD04.D

Instrument ID: D.i

Matrix: (SOIL/SED/WATER)

Level: (TRACE or LOW/MED)

GC Column: DB-624

$\frac{\text { TRACE }}{\text { ID: } 0.20 \quad(\mathrm{~mm})}$

Contract: $8 \mathrm{E}-00302$

SDG No.: 16407-1

\begin{tabular}{|c|c|c|c|}
\hline $\begin{array}{c}\text { EPA } \\
\text { SAMPLE NO. }\end{array}$ & $\begin{array}{r}\text { LAB } \\
\text { SAMPLE ID }\end{array}$ & $\begin{array}{c}\text { LAB } \\
\text { FILE ID }\end{array}$ & $\begin{array}{c}\text { TIME } \\
\text { ANALYZED }\end{array}$ \\
\hline $\begin{array}{l}\text { EVMW1-W-2982 } \\
3\end{array}$ & $200-16407-1$ & DJPD05.D & 1144 \\
\hline $\begin{array}{l}\text { EVMW 4-W-2982 } \\
6 \mathrm{DL}\end{array}$ & $200-16407-2$ & DJPD0 6.D & 1208 \\
\hline $\begin{array}{l}\text { EVMW 4-W-2982 } \\
6\end{array}$ & $200-16407-2$ & DJPD07.D & 1256 \\
\hline VIBLKDP & VIBLK 200-55472/8 & DJPD0 8.D & 1319 \\
\hline $\begin{array}{l}\text { EVSB } 63-W-298 \\
32\end{array}$ & $200-16407-3$ & DJPD09.D & 1343 \\
\hline $\begin{array}{l}\text { EVQCTB-W-298 } \\
42\end{array}$ & $200-16407-4$ & DJPD10.D & 1407 \\
\hline $\begin{array}{l}\text { EVSB } 80-W-298 \\
42\end{array}$ & $200-16407-5$ & DJPD11.D & 1431 \\
\hline VHBLK01 & $200-16407-6$ & DJPD12.D & 1454 \\
\hline
\end{tabular}

COMMENTS :

Page 1 of 1 
$5 A$ - FORM V VOA

VOLATILE ORGANICS INSTRUMENT

PERFORMANCE CHECK

BROMOFLUOROBENZENE
EPA SAMPLE NO.

BFBDG
Lab Name: TESTAMERICA BURLINGTON

Lab Code: STLV Case No.: EVERES Mod. Ref No.:
Contract: $8 \mathrm{E}-00302$

\begin{tabular}{|c|c|c|c|}
\hline $\mathrm{BEB}$ & Injection & Date: & $05 / 01 / 2013$ \\
\hline $\mathrm{BFB}$ & Injection & Time: & 1058 \\
\hline
\end{tabular}
$(\mathrm{mm})$

\begin{tabular}{|c|c|c|}
\hline $\mathrm{m} / \mathrm{e}$ & ION ABUNDANCE CRITERIA & $\begin{array}{l}\circ \text { RELATIVE } \\
\text { ABUNDANCE }\end{array}$ \\
\hline 50 & $15.0-40.0 \%$ of mass 95 & 16.4 \\
\hline 75 & $30.0-80.0 \%$ of mass 95 & 48.7 \\
\hline 95 & Base peak, $100 \%$ relative abundance & 100 \\
\hline 96 & $5.0-9.0 \%$ of mass 95 & 6.7 \\
\hline 173 & Less than 2.0\% of mass 174 & $0.5 \quad(0.6) 1$ \\
\hline 174 & $50.0-120 \%$ of mass 95 & 80.4 \\
\hline 175 & $5.0-9.0 \%$ of mass 174 & $5.6(6.9) 1$ \\
\hline 176 & $95.0-101 \%$ of mass 174 & $78.5(97.6) 1$ \\
\hline 177 & $5.0-9.0 \div$ of mass 176 & $5.1(6.5) 2$ \\
\hline
\end{tabular}

1 - Value is omass 174

2 - Value is omass 176

\begin{tabular}{c|c|c|l|c|c|}
\cline { 2 - 7 } & \multicolumn{1}{|c|}{$\begin{array}{c}\text { EPA } \\
\text { SAMPLE NO. }\end{array}$} & \multicolumn{1}{|c|}{ LAB } & LAB & DATE & TIME \\
01 & \multicolumn{1}{|c|}{ SAMPLE ID } & FILE ID & ANALYZED & ANALYZED \\
\cline { 2 - 7 } 02 & VSTD0.5DG & IC 200-55044/3 & DJP03.D & 05/01/2013 & 1132 \\
\cline { 2 - 7 } 03 & VSTD001DG & IC 200-55044/4 & DJP04.D & $05 / 01 / 2013$ & 1155 \\
\cline { 2 - 7 } 04 & VSTD005DG & ICIS 200-55044/5 & DJP05.D & $05 / 01 / 2013$ & 1219 \\
\cline { 2 - 7 } 05 & VSTD010DG & IC 200-55044/6 & DJP06.D & $05 / 01 / 2013$ & 1243 \\
\cline { 2 - 7 } & VSTD020DG & IC 200-55044/7 & DJP07.D & $05 / 01 / 2013$ & 1306 \\
\hline
\end{tabular}


$5 A$ - FORM V VOA

VOLATILE ORGANICS INSTRUMENT

PERFORMANCE CHECK

BROMOFLUOROBENZENE
EPA SAMPLE NO. BFBDL
Lab Name: TESTAMERICA BURLINGTON

Lab Code: STLV

Lab File Id: DJPD01.D

Instrument Id: D.i

GC Column: DB-624
Case No.: EVERES Mod. Ref No.:

Contract: $\quad$ 8E-00302

$\begin{array}{ll}\text { BFB Injection Date: } & \frac{05 / 13 / 2013}{1021} \\ \text { BFB Injection Time: } & \underline{1021}\end{array}$
$(\mathrm{mm})$

\begin{tabular}{|c|c|c|}
\hline $\mathrm{m} / \mathrm{e}$ & ION ABUNDANCE CRITERIA & $\begin{array}{l}\text { RELATIVE } \\
\text { ABUNDANCE }\end{array}$ \\
\hline 50 & $15.0-40.0 \%$ of mass 95 & 17.3 \\
\hline 75 & $30.0-80.0 \%$ of mass 95 & 49.6 \\
\hline 95 & Base peak, $100 \%$ relative abundance & 100 \\
\hline 96 & $5.0-9.0 \%$ of mass 95 & 6.7 \\
\hline 173 & Less than 2.0\% of mass 174 & $0.6) 1$ \\
\hline 174 & $50.0-120 \%$ of mass 95 & 74.4 \\
\hline 175 & $5.0-9.0 \%$ of mass 174 & 5.11 \\
\hline 176 & $95.0-101 \%$ of mass 174 & $71.6(96.3) 1$ \\
\hline 177 & $5.0-9.0 \%$ of mass 176 & $6.6) 2$ \\
\hline
\end{tabular}

1 - Value is omass 1742 - Value is omass 176

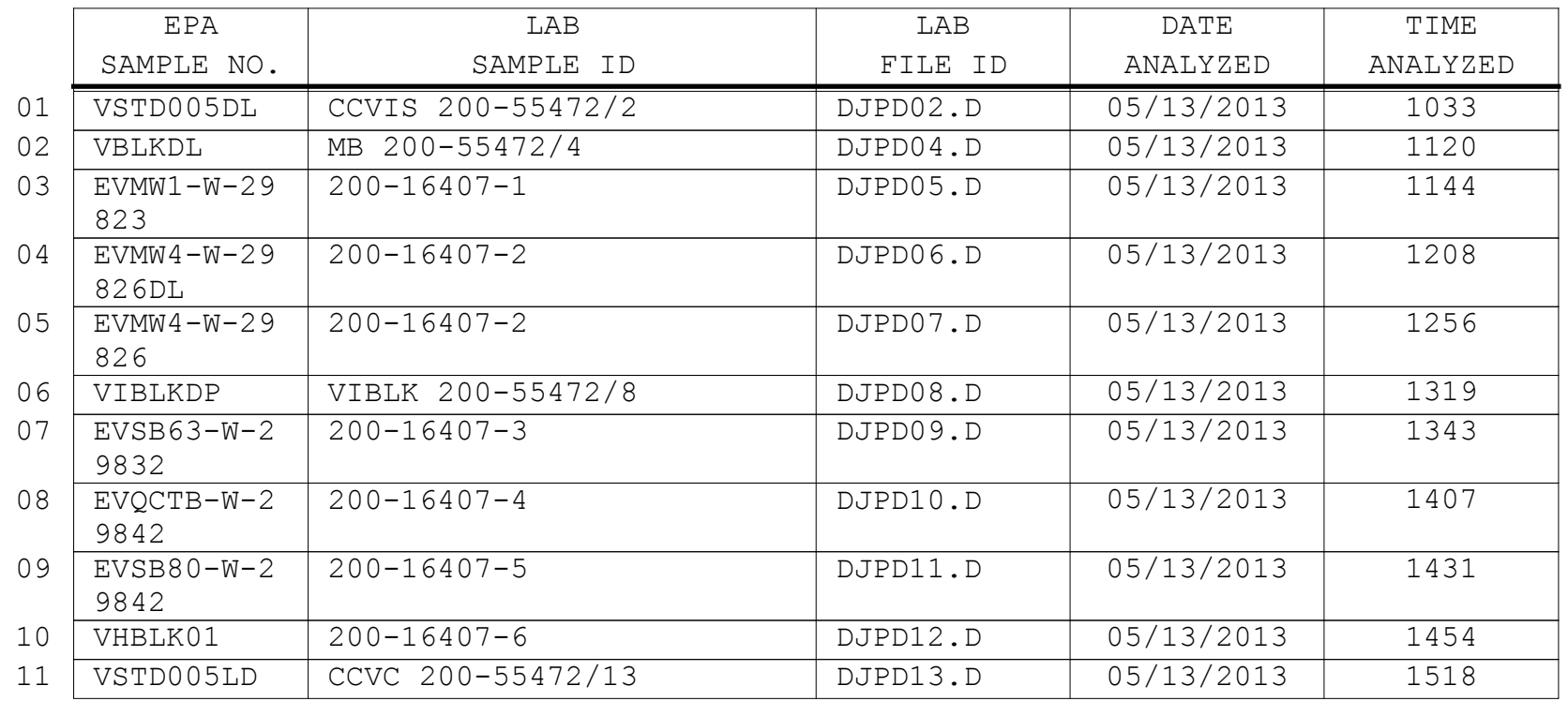




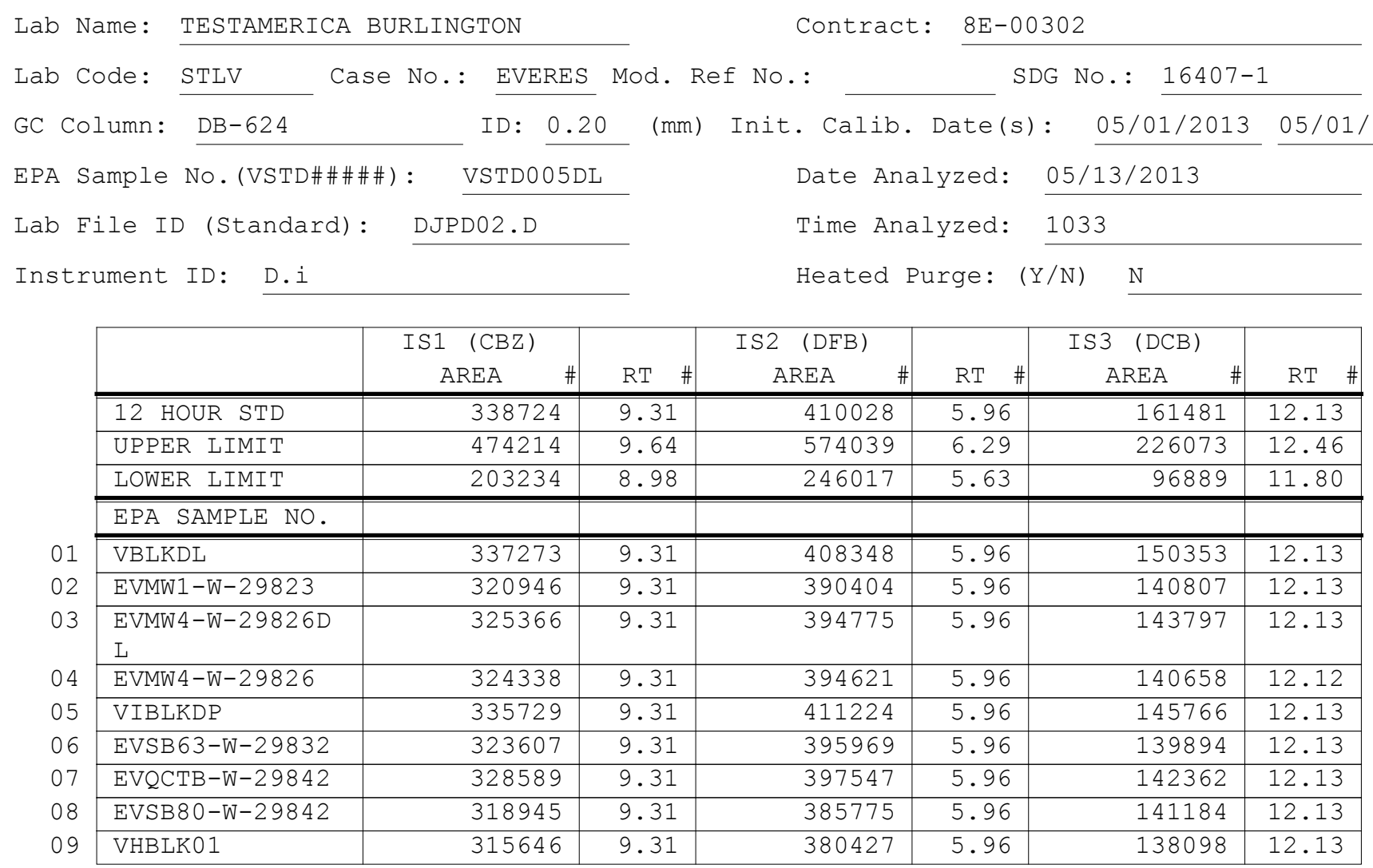

IS1 $(\mathrm{CBZ})=$ Chlorobenzene-d5

IS2 $(\mathrm{DFB})=1,4-\mathrm{Difluorobenzene}$

IS3 $(\mathrm{DCB})=1,4-\mathrm{Dichlorobenzene-d4}$

AREA UPPER LIMIT $=140 \%$ (Trace Volatiles) of internal standard area AREA LOWER LIMIT $=60 \%$ (Trace Volatiles) of internal standard area RT UPPER LIMIT $=+0.33$ (Trace Volatiles) minutes of internal standard RT $\mathrm{RT}$ LOWER LIMIT $=-0.33$ (Trace Volatiles) minutes of internal standard RT

\# Column used to flag values outside contract required QC limits with an asterisk. 
Lab Name: TESTAMERICA BURLINGTON

Lab Code: STLV Case No.: EVERES Mod. Ref No.:
Contract: 8E-00302

\section{Matrix: (SOIL/SED/WATER)}

Water

Sample wt/vol: 25.0 $(\mathrm{g} / \mathrm{mL}) \quad \mathrm{mL}$

Level: (TRACE/LOW/MED) TRACE

\% Moisture: not dec.

GC Column: DB-624 ID $: 0.20 \quad(\mathrm{~mm})$

Soil Extract Volume: (uL) $(\mathrm{mL})$

Purge Volume: 25.0

Lab File ID: DJPD05.D

Dilution Factor: 1.0

Soil Aliquot Volume: SDG No.: 16407-1 Lab Sample ID: 200-16407-1

Date Received: 05/10/2013

Date Analyzed: 05/13/2013

Purge Volume: 25.0

\begin{tabular}{|c|c|c|c|}
\hline CAS NO. & COMPOUND & $\begin{array}{l}\text { CONCENTRATION UNITS: } \\
(\mathrm{ug} / \mathrm{L} \text { or } \mathrm{ug} / \mathrm{kg}) \mathrm{ug} / \mathrm{L}\end{array}$ & Q \\
\hline $75-71-8$ & Dichlorodifluoromethane & 0.50 & $\bar{U}$ \\
\hline $74-87-3$ & Chloromethane & 0.50 & $\mathrm{U}$ \\
\hline $75-01-4$ & Vinyl chloride & 0.50 & $\mathrm{U}$ \\
\hline $74-83-9$ & Bromomethane & 0.50 & $\mathrm{U}$ \\
\hline $75-00-3$ & Chloroethane & 0.50 & $\mathrm{U}$ \\
\hline $75-69-4$ & Trichlorofluoromethane & 0.50 & $\mathrm{U}$ \\
\hline $75-35-4$ & 1,1-Dichloroethene & 0.50 & $\mathrm{U}$ \\
\hline $76-13-1$ & 1,1,2-Trichloro-1,2,2-trifluoroethane & 0.50 & $\mathrm{U}$ \\
\hline $67-64-1$ & Acetone & 5.0 & $\mathrm{U}$ \\
\hline $75-15-0$ & Carbon disulfide & 0.021 & $\mathrm{~J}$ \\
\hline $79-20-9$ & Methyl acetate & 0.50 & $\mathrm{U}$ \\
\hline $75-09-2$ & Methylene Chloride & 0.50 & $\mathrm{U}$ \\
\hline $156-60-5$ & trans-1,2-Dichloroethene & 0.50 & $\mathrm{U}$ \\
\hline $1634-04-4$ & Methyl tert-butyl ether & 0.50 & $\mathrm{U}$ \\
\hline $75-34-3$ & 1,1-Dichloroethane & 0.50 & $\mathrm{U}$ \\
\hline $156-59-2$ & cis-1,2-Dichloroethene & 0.50 & $\mathrm{U}$ \\
\hline $78-93-3$ & 2-Butanone & 5.0 & $\mathrm{U}$ \\
\hline $74-97-5$ & Bromochloromethane & 0.50 & $\mathrm{U}$ \\
\hline $67-66-3$ & Chloroform & 0.65 & \\
\hline $71-55-6$ & 1,1,1-Trichloroethane & 0.50 & $\mathrm{U}$ \\
\hline $110-82-7$ & Cyclohexane & 0.50 & $\mathrm{U}$ \\
\hline $56-23-5$ & Carbon tetrachloride & 3.9 & \\
\hline $71-43-2$ & Benzene & 0.50 & $\mathrm{U}$ \\
\hline $107-06-2$ & 1,2-Dichloroethane & 0.50 & $\mathrm{U}$ \\
\hline
\end{tabular}

Report 1,4-Dioxane for Low-Medium VOA analysis only 
Lab Name: TESTAMERICA BURLINGTON

Lab Code: STLV Case No.: EVERES Mod. Ref No.:
Contract: 8E-00302 SDG No.: 16407-1
Matrix: (SOIL/SED/WATER)

Water

Sample wt/vol: 25.0 $(\mathrm{g} / \mathrm{mL}) \quad \mathrm{mL}$

Level: (TRACE/LOW/MED) TRACE

\% Moisture: not dec.

GC Column: DB-624

Soil Extract Volume: ID $: 0.20 \quad(\mathrm{~mm})$

Purge Volume: 25.0
(uL)

$(\mathrm{mL})$
Lab Sample ID: 200-16407-1

Lab File ID: DJPD05.D

Date Received: 05/10/2013

Date Analyzed: 05/13/2013

Dilution Factor: 1.0

Soil Aliquot Volume: $(u L)$

\begin{tabular}{|c|c|c|c|}
\hline CAS NO. & COMPOUND & $\begin{array}{l}\text { CONCENTRATION UNITS: } \\
(\mathrm{ug} / \mathrm{L} \text { or } \mathrm{ug} / \mathrm{kg}) \mathrm{ug} / \mathrm{L}\end{array}$ & \& \\
\hline $79-01-6$ & Trichloroethene & 0.50 & $\overline{\mathrm{U}}$ \\
\hline $108-87-2$ & Methylcyclohexane & 0.50 & $\mathrm{U}$ \\
\hline $78-87-5$ & 1,2-Dichloropropane & 0.50 & $\mathrm{U}$ \\
\hline $75-27-4$ & Bromodichloromethane & 0.50 & $\mathrm{U}$ \\
\hline $10061-01-5$ & cis-1,3-Dichloropropene & 0.50 & $\mathrm{U}$ \\
\hline $108-10-1$ & 4-Methyl-2-pentanone & 5.0 & $\mathrm{U}$ \\
\hline $108-88-3$ & Toluene & 0.50 & $\mathrm{U}$ \\
\hline $10061-02-6$ & trans-1,3-Dichloropropene & 0.50 & $\mathrm{U}$ \\
\hline $79-00-5$ & $1,1,2$-Trichloroethane & 0.50 & $\mathrm{U}$ \\
\hline $127-18-4$ & Tetrachloroethene & 0.50 & $\mathrm{U}$ \\
\hline $591-78-6$ & 2-Hexanone & 5.0 & $\mathrm{U}$ \\
\hline $124-48-1$ & Dibromochloromethane & 0.50 & $\mathrm{U}$ \\
\hline $106-93-4$ & 1,2-Dibromoethane & 0.50 & $\mathrm{U}$ \\
\hline $108-90-7$ & Chlorobenzene & 0.50 & $\mathrm{U}$ \\
\hline $100-41-4$ & Ethylbenzene & 0.0073 & $\mathrm{~J}$ \\
\hline $95-47-6$ & o-Xylene & 0.0049 & $\mathrm{~J}$ \\
\hline $179601-23-1$ & $\mathrm{~m}, \mathrm{p}-\mathrm{Xyl}$ lene & 0.030 & $\mathrm{~J}$ \\
\hline $100-42-5$ & Styrene & 0.50 & $\mathrm{U}$ \\
\hline $75-25-2$ & Bromoform & 0.50 & $\mathrm{U}$ \\
\hline $98-82-8$ & Isopropylbenzene & 0.50 & $\mathrm{U}$ \\
\hline $79-34-5$ & $1,1,2,2$-Tetrachloroethane & 0.50 & $\mathrm{U}$ \\
\hline $541-73-1$ & 1,3-Dichlorobenzene & 0.50 & $\mathrm{U}$ \\
\hline $106-46-7$ & 1,4-Dichlorobenzene & 0.50 & $\mathrm{U}$ \\
\hline $95-50-1$ & 1,2-Dichlorobenzene & 0.50 & $\mathrm{U}$ \\
\hline $96-12-8$ & 1,2-Dibromo-3-Chloropropane & 0.50 & $\mathrm{U}$ \\
\hline $120-82-1$ & 1,2,4-Trichlorobenzene & 0.50 & $\mathrm{U}$ \\
\hline $87-61-6$ & $1,2,3$-Trichlorobenzene & 0.50 & $\mathrm{U}$ \\
\hline
\end{tabular}


$1 \mathrm{~J}$ - FORM I VOA-TIC

VOLATILE ORGANICS ANALYSIS DATA SHEET

TENTATIVELY IDENTIFIED COMPOUNDS
EPA SAMPLE NO.

EVMW1-W-29823

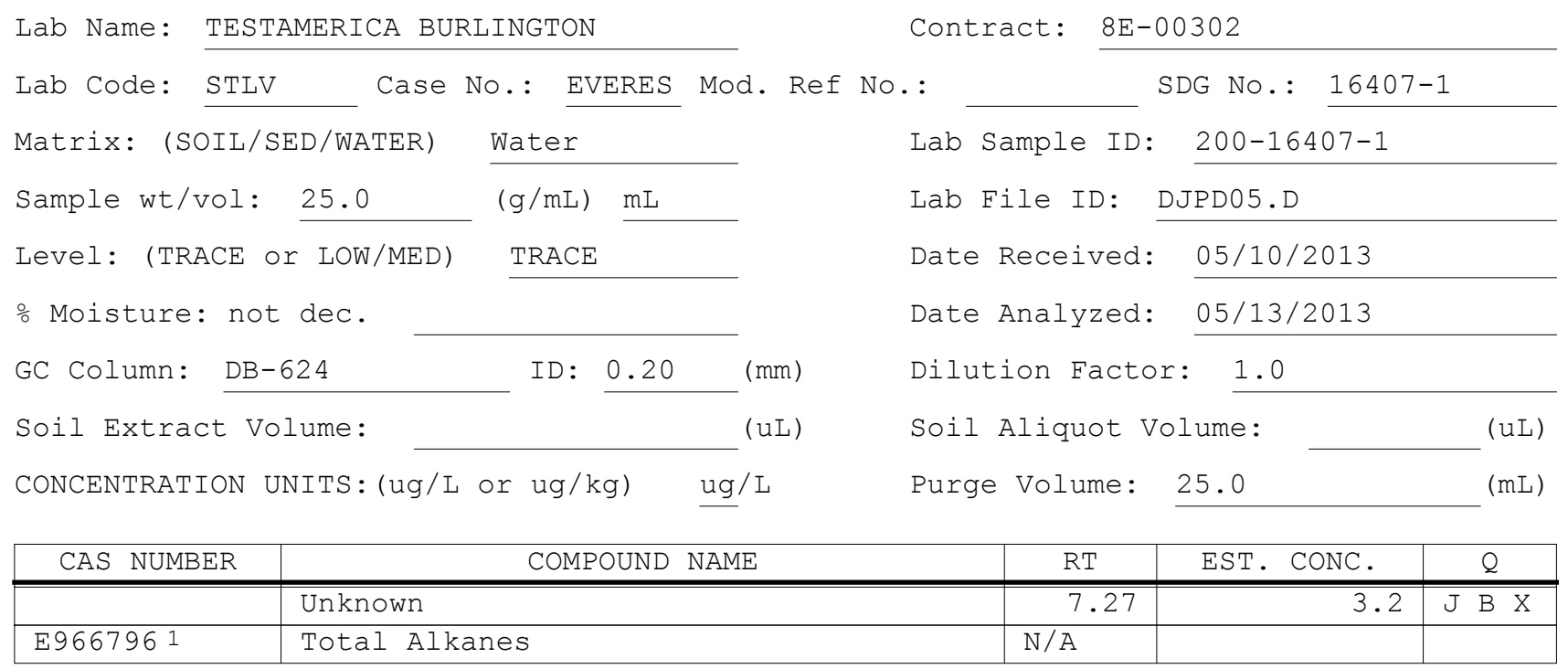

1 EPA-designated Registry Number. 
Lab Name: TESTAMERICA BURLINGTON

Lab Code: STLV Case No.: EVERES Mod. Ref No.:
Contract: 8E-00302

\section{Matrix: (SOIL/SED/WATER)}

Water

Sample wt/vol: 25.0 $(\mathrm{g} / \mathrm{mL}) \quad \mathrm{mL}$

Level: (TRACE/LOW/MED) TRACE

\% Moisture: not dec.

GC Column: DB-624 ID $: 0.20 \quad(\mathrm{~mm})$

Soil Extract Volume: (uL) $(\mathrm{mL})$

Purge Volume: 25.0 SDG No.: 16407-1

Lab Sample ID: 200-16407-2

Lab File ID: DJPD07.D

Date Received: 05/10/2013

Date Analyzed: 05/13/2013

Dilution Factor: 1.0

Soil Aliquot Volume: (uL)

\begin{tabular}{|c|c|c|c|}
\hline CAS NO. & COMPOUND & $\begin{array}{l}\text { CONCENTRATION UNITS: } \\
(\mathrm{ug} / \mathrm{L} \text { or } \mathrm{ug} / \mathrm{kg}) \mathrm{ug} / \mathrm{L}\end{array}$ & Q \\
\hline $75-71-8$ & Dichlorodifluoromethane & 0.50 & $\mathrm{U}$ \\
\hline $74-87-3$ & Chloromethane & 0.50 & $\mathrm{U}$ \\
\hline $75-01-4$ & Vinyl chloride & 0.50 & $\mathrm{U}$ \\
\hline $74-83-9$ & Bromomethane & 0.50 & $\mathrm{U}$ \\
\hline $75-00-3$ & Chloroethane & 0.50 & $\mathrm{U}$ \\
\hline $75-69-4$ & Trichlorofluoromethane & 0.50 & $\mathrm{U}$ \\
\hline $75-35-4$ & 1,1-Dichloroethene & 0.50 & $\mathrm{U}$ \\
\hline $76-13-1$ & 1,1,2-Trichloro-1,2,2-trifluoroethane & 0.50 & $\mathrm{U}$ \\
\hline $67-64-1$ & Acetone & 5.0 & $\mathrm{U}$ \\
\hline $75-15-0$ & Carbon disulfide & 0.50 & $\mathrm{U}$ \\
\hline $79-20-9$ & Methyl acetate & 0.50 & $\mathrm{U}$ \\
\hline $75-09-2$ & Methylene Chloride & 0.50 & $\mathrm{U}$ \\
\hline $156-60-5$ & trans-1,2-Dichloroethene & 0.50 & $\mathrm{U}$ \\
\hline $1634-04-4$ & Methyl tert-butyl ether & 0.50 & $\mathrm{U}$ \\
\hline $75-34-3$ & 1,1-Dichloroethane & 0.50 & $\mathrm{U}$ \\
\hline $156-59-2$ & cis-1,2-Dichloroethene & 0.50 & $\mathrm{U}$ \\
\hline $78-93-3$ & 2-Butanone & 5.0 & $\mathrm{U}$ \\
\hline $74-97-5$ & Bromochloromethane & 0.50 & $\mathrm{U}$ \\
\hline $67-66-3$ & Chloroform & 2.8 & \\
\hline $71-55-6$ & $1,1,1$-Trichloroethane & 0.50 & $\mathrm{U}$ \\
\hline $110-82-7$ & Cyclohexane & 0.50 & $\mathrm{U}$ \\
\hline $56-23-5$ & Carbon tetrachloride & 89 & $E$ \\
\hline $71-43-2$ & Benzene & 0.50 & $\mathrm{U}$ \\
\hline $107-06-2$ & 1,2-Dichloroethane & 0.50 & $\mathrm{U}$ \\
\hline
\end{tabular}

Report 1,4-Dioxane for Low-Medium VOA analysis only 
Lab Name: TESTAMERICA BURLINGTON

Lab Code: STLV Case No.: EVERES Mod. Ref No.:
Contract: 8E-00302 SDG No.: 16407-1
Matrix: (SOIL/SED/WATER)

Water

Sample wt/vol: 25.0 $(\mathrm{g} / \mathrm{mL}) \quad \mathrm{mL}$

Level : (TRACE/LOW/MED)

TRACE

\% Moisture: not dec.

GC Column: DB-624

Soil Extract Volume: ID $: 0.20 \quad(\mathrm{~mm})$

Purge Volume: 25.0
(uL)

$(\mathrm{mL})$
Lab Sample ID: 200-16407-2

Lab File ID: DJPD07.D

Date Received: 05/10/2013

Date Analyzed: 05/13/2013

Dilution Factor: 1.0

Soil Aliquot Volume: $(u L)$

\begin{tabular}{|c|c|c|c|}
\hline CAS NO. & COMPOUND & $\begin{array}{l}\text { CONCENTRATION UNITS: } \\
(\mathrm{ug} / \mathrm{L} \text { or } \mathrm{ug} / \mathrm{kg}) \mathrm{ug} / \mathrm{L}\end{array}$ & \& \\
\hline $79-01-6$ & Trichloroethene & 0.50 & $\overline{\mathrm{U}}$ \\
\hline $108-87-2$ & Methylcyclohexane & 0.50 & $\mathrm{U}$ \\
\hline $78-87-5$ & 1,2-Dichloropropane & 0.50 & $\mathrm{U}$ \\
\hline $75-27-4$ & Bromodichloromethane & 0.50 & $\mathrm{U}$ \\
\hline $10061-01-5$ & cis-1,3-Dichloropropene & 0.50 & $\mathrm{U}$ \\
\hline $108-10-1$ & 4-Methyl-2-pentanone & 5.0 & $\mathrm{U}$ \\
\hline $108-88-3$ & Toluene & 0.50 & $\mathrm{U}$ \\
\hline $10061-02-6$ & trans-1,3-Dichloropropene & 0.50 & $\mathrm{U}$ \\
\hline $79-00-5$ & $1,1,2$-Trichloroethane & 0.50 & $\mathrm{U}$ \\
\hline $127-18-4$ & Tetrachloroethene & 0.50 & $\mathrm{U}$ \\
\hline $591-78-6$ & 2-Hexanone & 5.0 & $\mathrm{U}$ \\
\hline $124-48-1$ & Dibromochloromethane & 0.50 & $\mathrm{U}$ \\
\hline $106-93-4$ & 1,2-Dibromoethane & 0.50 & $\mathrm{U}$ \\
\hline $108-90-7$ & Chlorobenzene & 0.50 & $\mathrm{U}$ \\
\hline $100-41-4$ & Ethylbenzene & 0.0078 & $\mathrm{~J}$ \\
\hline $95-47-6$ & o-Xylene & 0.0038 & $\mathrm{~J}$ \\
\hline $179601-23-1$ & $\mathrm{~m}, \mathrm{p}$-Xylene & 0.028 & $\mathrm{~J}$ \\
\hline $100-42-5$ & Styrene & 0.50 & $\mathrm{U}$ \\
\hline $75-25-2$ & Bromoform & 0.50 & $\mathrm{U}$ \\
\hline $98-82-8$ & Isopropylbenzene & 0.50 & $\mathrm{U}$ \\
\hline $79-34-5$ & $1,1,2,2$-Tetrachloroethane & 0.50 & $\mathrm{U}$ \\
\hline $541-73-1$ & 1,3-Dichlorobenzene & 0.50 & $\mathrm{U}$ \\
\hline $106-46-7$ & 1,4-Dichlorobenzene & 0.50 & $\mathrm{U}$ \\
\hline $95-50-1$ & 1,2-Dichlorobenzene & 0.50 & $\mathrm{U}$ \\
\hline $96-12-8$ & 1,2-Dibromo-3-Chloropropane & 0.50 & $\mathrm{U}$ \\
\hline $120-82-1$ & 1,2,4-Trichlorobenzene & 0.50 & $\mathrm{U}$ \\
\hline $87-61-6$ & $1,2,3$-Trichlorobenzene & 0.50 & $\mathrm{U}$ \\
\hline
\end{tabular}


$1 \mathrm{~J}$ - FORM I VOA-TIC

VOLATILE ORGANICS ANALYSIS DATA SHEET

TENTATIVELY IDENTIFIED COMPOUNDS
EPA SAMPLE NO.

EVMW4-W-29826

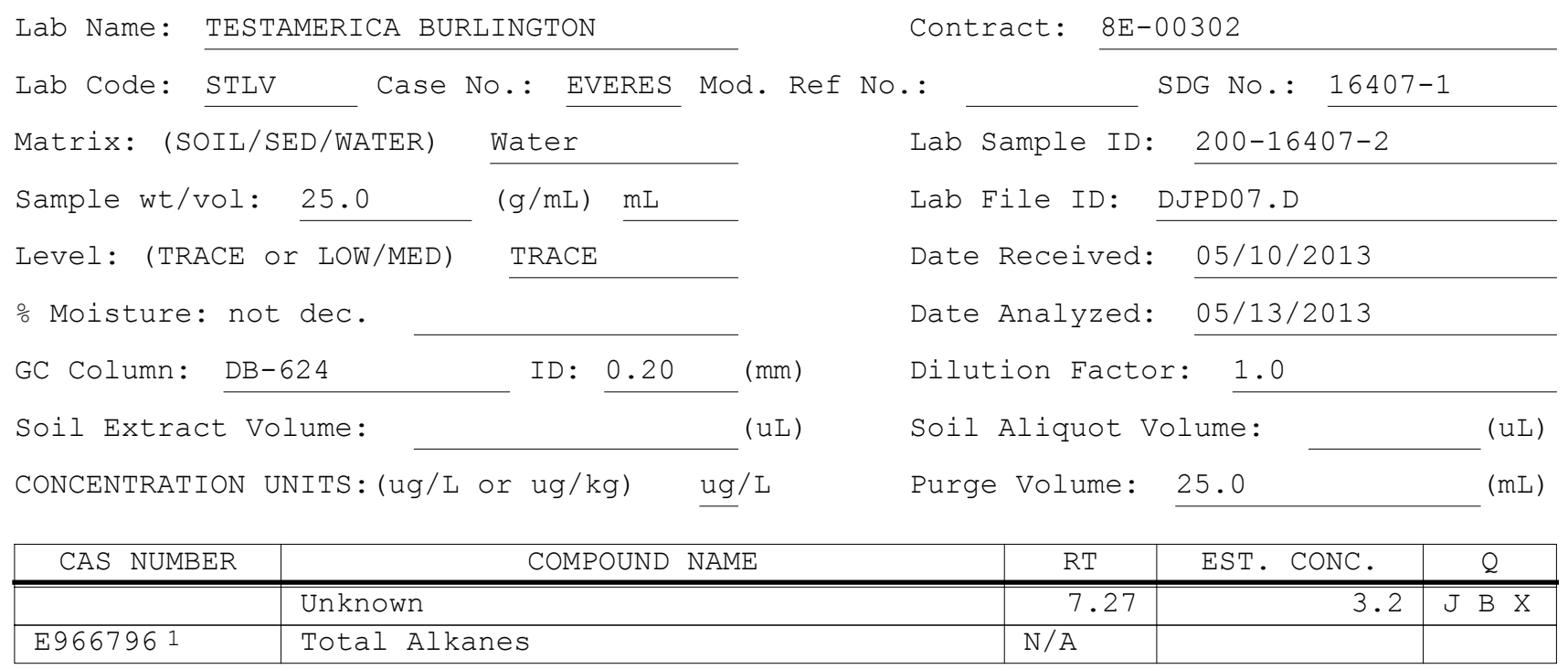

1 EPA-designated Registry Number. 
Lab Name: TESTAMERICA BURLINGTON

Contract: $\quad 8 \mathrm{E}-00302$

Lab Code: STLV Case No.: EVERES Mod. Ref No.: SDG No.: 16407-1

Matrix: (SOIL/SED/WATER)

Water

Sample wt/vol: 25.0 $(\mathrm{g} / \mathrm{mL}) \quad \mathrm{mL}$

Level: (TRACE/LOW/MED) TRACE

\% Moisture: not dec.

GC Column: DB-624

Soil Extract Volume: ID $: 0.20 \quad(\mathrm{~mm})$

Purge Volume: 25.0
Lab Sample ID: 200-16407-2

Lab File ID: DJPD06.D

Date Received: 05/10/2013

Date Analyzed: 05/13/2013

Dilution Factor: 4.7

Soil Aliquot Volume: (uL)

\begin{tabular}{|c|c|c|c|}
\hline CAS NO. & COMPOUND & $\begin{array}{l}\text { CONCENTRATION UNITS: } \\
(\mathrm{ug} / \mathrm{L} \text { or } \mathrm{ug} / \mathrm{kg}) \mathrm{ug} / \mathrm{L}\end{array}$ & Q \\
\hline $75-71-8$ & Dichlorodifluoromethane & 2.4 & $\overline{\mathrm{U}}$ \\
\hline $74-87-3$ & Chloromethane & 2.4 & $\mathrm{U}$ \\
\hline $75-01-4$ & Vinyl chloride & 2.4 & U \\
\hline $74-83-9$ & Bromomethane & 2.4 & U \\
\hline $75-00-3$ & Chloroethane & 2.4 & $\mathrm{U}$ \\
\hline $75-69-4$ & Trichlorofluoromethane & 2.4 & $\mathrm{U}$ \\
\hline $75-35-4$ & 1,1-Dichloroethene & 2.4 & $\mathrm{U}$ \\
\hline $76-13-1$ & 1,1,2-Trichloro-1,2,2-trifluoroethane & 2.4 & $\mathrm{U}$ \\
\hline $67-64-1$ & Acetone & 24 & $\mathrm{U}$ \\
\hline $75-15-0$ & Carbon disulfide & 2.4 & $\mathrm{U}$ \\
\hline $79-20-9$ & Methyl acetate & 2.4 & $\mathrm{U}$ \\
\hline $75-09-2$ & Methylene Chloride & 0.73 & $\mathrm{~J} B \mathrm{D}$ \\
\hline $156-60-5$ & trans-1,2-Dichloroethene & 2.4 & $\mathrm{U}$ \\
\hline $1634-04-4$ & Methyl tert-butyl ether & 2.4 & $\mathrm{U}$ \\
\hline $75-34-3$ & 1,1-Dichloroethane & 2.4 & $\mathrm{U}$ \\
\hline $156-59-2$ & cis-1,2-Dichloroethene & 2.4 & $\mathrm{U}$ \\
\hline $78-93-3$ & 2-Butanone & 24 & U \\
\hline $74-97-5$ & Bromochloromethane & 2.4 & U \\
\hline $67-66-3$ & Chloroform & 2.9 & $\mathrm{D}$ \\
\hline $71-55-6$ & $1,1,1$-Trichloroethane & 2.4 & $\mathrm{U}$ \\
\hline $110-82-7$ & Cyclohexane & 2.4 & $\mathrm{U}$ \\
\hline $56-23-5$ & Carbon tetrachloride & 78 & $\mathrm{D}$ \\
\hline $71-43-2$ & Benzene & 2.4 & $\mathrm{U}$ \\
\hline $107-06-2$ & 1,2-Dichloroethane & 2.4 & $\mathrm{U}$ \\
\hline
\end{tabular}

Report 1,4-Dioxane for Low-Medium VOA analysis only 
Lab Name: TESTAMERICA BURLINGTON

Lab Code: STLV Case No.: EVERES Mod. Ref No.:
Contract: 8E-00302 SDG No.: 16407-1
Matrix: (SOIL/SED/WATER)

Water

Sample wt/vol: 25.0 $(\mathrm{g} / \mathrm{mL}) \quad \mathrm{mL}$

Level : (TRACE/LOW/MED)

TRACE

\% Moisture: not dec.

GC Column: DB-624

Soil Extract Volume: ID $: 0.20 \quad(\mathrm{~mm})$

Purge Volume: 25.0
(uL)

$(\mathrm{mL})$
Lab Sample ID: 200-16407-2

Lab File ID: DJPD06.D

Date Received: 05/10/2013

Date Analyzed: 05/13/2013

Dilution Factor: 4.7

Soil Aliquot Volume: (uL)

\begin{tabular}{|c|c|c|c|}
\hline CAS NO. & COMPOUND & $\begin{array}{l}\text { CONCENTRATION UNITS: } \\
(\mathrm{ug} / \mathrm{L} \text { or } \mathrm{ug} / \mathrm{kg}) \mathrm{ug} / \mathrm{L}\end{array}$ & \& \\
\hline $79-01-6$ & Trichloroethene & 2.4 & $\overline{\mathrm{U}}$ \\
\hline $108-87-2$ & Methylcyclohexane & 2.4 & $\mathrm{U}$ \\
\hline $78-87-5$ & 1,2-Dichloropropane & 2.4 & $\mathrm{U}$ \\
\hline $75-27-4$ & Bromodichloromethane & 2.4 & U \\
\hline $10061-01-5$ & cis-1,3-Dichloropropene & 2.4 & $\mathrm{U}$ \\
\hline $108-10-1$ & 4-Methyl-2-pentanone & 24 & $\mathrm{U}$ \\
\hline $108-88-3$ & Toluene & 2.4 & $\mathrm{U}$ \\
\hline $10061-02-6$ & trans-1,3-Dichloropropene & 2.4 & $\mathrm{U}$ \\
\hline $79-00-5$ & $1,1,2$-Trichloroethane & 2.4 & $\mathrm{U}$ \\
\hline $127-18-4$ & Tetrachloroethene & 2.4 & $\mathrm{U}$ \\
\hline $591-78-6$ & 2-Hexanone & 24 & $\mathrm{U}$ \\
\hline $124-48-1$ & Dibromochloromethane & 2.4 & $\mathrm{U}$ \\
\hline $106-93-4$ & 1,2-Dibromoethane & 2.4 & $\mathrm{U}$ \\
\hline $108-90-7$ & Chlorobenzene & 2.4 & $\mathrm{U}$ \\
\hline $100-41-4$ & Ethylbenzene & 2.4 & $\mathrm{U}$ \\
\hline $95-47-6$ & o-Xylene & 2.4 & $\mathrm{U}$ \\
\hline $179601-23-1$ & $\mathrm{~m}, \mathrm{p}$-Xylene & 2.4 & $\mathrm{U}$ \\
\hline $100-42-5$ & Styrene & 2.4 & $\mathrm{U}$ \\
\hline $75-25-2$ & Bromoform & 2.4 & $\mathrm{U}$ \\
\hline $98-82-8$ & Isopropylbenzene & 2.4 & $\mathrm{U}$ \\
\hline $79-34-5$ & $1,1,2,2$-Tetrachloroethane & 2.4 & $\mathrm{U}$ \\
\hline $541-73-1$ & 1,3-Dichlorobenzene & 2.4 & $\mathrm{U}$ \\
\hline $106-46-7$ & 1,4-Dichlorobenzene & 2.4 & $\mathrm{U}$ \\
\hline $95-50-1$ & 1,2-Dichlorobenzene & 2.4 & $\mathrm{U}$ \\
\hline $96-12-8$ & 1,2-Dibromo-3-Chloropropane & 2.4 & $\mathrm{U}$ \\
\hline $120-82-1$ & 1,2,4-Trichlorobenzene & 2.4 & $\mathrm{U}$ \\
\hline $87-61-6$ & $1,2,3$-Trichlorobenzene & 2.4 & $\mathrm{U}$ \\
\hline
\end{tabular}


$1 \mathrm{~J}$ - FORM I VOA-TIC

VOLATILE ORGANICS ANALYSIS DATA SHEET

TENTATIVELY IDENTIFIED COMPOUNDS
EPA SAMPLE NO.

EVMW4-W-29826DL

\begin{tabular}{|c|c|c|c|c|c|}
\hline Lab Name: TESTAMERICA BU & RLINGTON & & Contract: & & \\
\hline Lab Code: & EVERES & Mod. & SDG No.: & \multicolumn{2}{|l|}{$16407-1$} \\
\hline Matrix: (SOIL/SED/WATER) & \multicolumn{2}{|l|}{ Water } & Lab Sample ID: & $200-16407-2$ & \\
\hline Sample wt/vol: 25.0 & $(\mathrm{~g} / \mathrm{mL})$ & & Lab File ID: & DJPD06.D & \\
\hline Level: (TRACE or LOW/MED) & TRACE & & Date Received: & 2013 & \\
\hline \% Moisture: not dec. & & & Date Analyzed: & 2013 & \\
\hline GC Column: & 0.20 & $(\mathrm{~mm})$ & Dilution Factor: & & \\
\hline Soil Extract Volume: & & $(u L)$ & Soil Aliquot Volume: & & $(u L)$ \\
\hline CONCENTRATION UNITS: (ug/I & or ug/kg) & $\mathrm{ug} / \mathrm{L}$ & Purge Volume: & & $(\mathrm{mL})$ \\
\hline
\end{tabular}

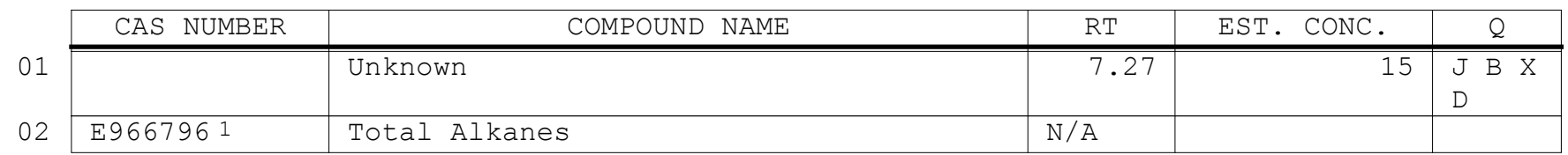

1 EPA-designated Registry Number. 
Lab Name: TESTAMERICA BURLINGTON

Contract: $\quad 8 \mathrm{E}-00302$

Lab Code: STLV Case No.: EVERES Mod. Ref No.:

Matrix: (SOIL/SED/WATER)

Water

Sample wt/vol: 25.0 $(\mathrm{g} / \mathrm{mL}) \quad \mathrm{mL}$

Level: (TRACE/LOW/MED) TRACE

\% Moisture: not dec.

GC Column: DB-624

Soil Extract Volume: ID $: 0.20 \quad(\mathrm{~mm})$

Purge Volume: 25.0
: $\quad$ SDG No.: 16407-1

Lab Sample ID: 200-16407-4

Lab File ID: DJPD10.D

Date Received: 05/10/2013

Date Analyzed: 05/13/2013

Dilution Factor: 1.0

Soil Aliquot Volume:

\begin{tabular}{|c|c|c|c|}
\hline CAS NO. & COMPOUND & $\begin{array}{l}\text { CONCENTRATION UNITS: } \\
(\mathrm{ug} / \mathrm{L} \text { or } \mathrm{ug} / \mathrm{kg}) \mathrm{ug} / \mathrm{L}\end{array}$ & Q \\
\hline $75-71-8$ & Dichlorodifluoromethane & 0.50 & $\bar{U}$ \\
\hline $74-87-3$ & Chloromethane & 0.50 & $\mathrm{U}$ \\
\hline $75-01-4$ & Vinyl chloride & 0.50 & $\mathrm{U}$ \\
\hline $74-83-9$ & Bromomethane & 0.50 & $\mathrm{U}$ \\
\hline $75-00-3$ & Chloroethane & 0.50 & $\mathrm{U}$ \\
\hline $75-69-4$ & Trichlorofluoromethane & 0.50 & $\mathrm{U}$ \\
\hline $75-35-4$ & 1,1-Dichloroethene & 0.50 & $\mathrm{U}$ \\
\hline $76-13-1$ & 1,1,2-Trichloro-1,2,2-trifluoroethane & 0.50 & $\mathrm{U}$ \\
\hline $67-64-1$ & Acetone & 4.1 & $\mathrm{~J}$ \\
\hline $75-15-0$ & Carbon disulfide & 0.50 & $\mathrm{U}$ \\
\hline $79-20-9$ & Methyl acetate & 0.50 & $\mathrm{U}$ \\
\hline $75-09-2$ & Methylene Chloride & 0.50 & $\mathrm{U}$ \\
\hline $156-60-5$ & trans-1,2-Dichloroethene & 0.50 & $\mathrm{U}$ \\
\hline $1634-04-4$ & Methyl tert-butyl ether & 0.50 & $\mathrm{U}$ \\
\hline $75-34-3$ & 1,1-Dichloroethane & 0.50 & $\mathrm{U}$ \\
\hline $156-59-2$ & cis-1,2-Dichloroethene & 0.50 & $\mathrm{U}$ \\
\hline $78-93-3$ & 2-Butanone & 5.0 & $\mathrm{U}$ \\
\hline $74-97-5$ & Bromochloromethane & 0.50 & $\mathrm{U}$ \\
\hline $67-66-3$ & Chloroform & 0.50 & $\mathrm{U}$ \\
\hline $71-55-6$ & 1,1,1-Trichloroethane & 0.50 & $\mathrm{U}$ \\
\hline $110-82-7$ & Cyclohexane & 0.50 & $\mathrm{U}$ \\
\hline $56-23-5$ & Carbon tetrachloride & 0.50 & $\mathrm{U}$ \\
\hline $71-43-2$ & Benzene & 0.50 & $\mathrm{U}$ \\
\hline $107-06-2$ & 1,2-Dichloroethane & 0.50 & $\mathrm{U}$ \\
\hline
\end{tabular}

Report 1,4-Dioxane for Low-Medium VOA analysis only 
Lab Name: TESTAMERICA BURLINGTON

Lab Code: STLV Case No.: EVERES Mod. Ref No.:
Contract: 8E-00302 SDG No.: 16407-1
Matrix: (SOIL/SED/WATER)

Water

Sample wt/vol: 25.0 $(\mathrm{g} / \mathrm{mL}) \quad \mathrm{mL}$

Level : (TRACE/LOW/MED)

TRACE

\% Moisture: not dec.

GC Column: DB-624

Soil Extract Volume: ID $: 0.20 \quad(\mathrm{~mm})$

Purge Volume: 25.0
(uL)

$(\mathrm{mL})$
Lab Sample ID: 200-16407-4

Lab File ID: DJPD10.D

Date Received: 05/10/2013

Date Analyzed: 05/13/2013

Dilution Factor: 1.0

Soil Aliquot Volume: $(u L)$

\begin{tabular}{|c|c|c|c|}
\hline CAS NO. & COMPOUND & $\begin{array}{l}\text { CONCENTRATION UNITS: } \\
(\mathrm{ug} / \mathrm{L} \text { or } \mathrm{ug} / \mathrm{kg}) \mathrm{ug} / \mathrm{L}\end{array}$ & \& \\
\hline $79-01-6$ & Trichloroethene & 0.50 & $\overline{\mathrm{U}}$ \\
\hline $108-87-2$ & Methylcyclohexane & 0.50 & $\mathrm{U}$ \\
\hline $78-87-5$ & 1,2-Dichloropropane & 0.50 & $\mathrm{U}$ \\
\hline $75-27-4$ & Bromodichloromethane & 0.50 & $\mathrm{U}$ \\
\hline $10061-01-5$ & cis-1,3-Dichloropropene & 0.50 & $\mathrm{U}$ \\
\hline $108-10-1$ & 4-Methyl-2-pentanone & 5.0 & $\mathrm{U}$ \\
\hline $108-88-3$ & Toluene & 0.40 & $\mathrm{~J}$ \\
\hline $10061-02-6$ & trans-1,3-Dichloropropene & 0.50 & $\mathrm{U}$ \\
\hline $79-00-5$ & $1,1,2$-Trichloroethane & 0.50 & $\mathrm{U}$ \\
\hline $127-18-4$ & Tetrachloroethene & 0.50 & $\mathrm{U}$ \\
\hline $591-78-6$ & 2-Hexanone & 5.0 & $\mathrm{U}$ \\
\hline $124-48-1$ & Dibromochloromethane & 0.50 & $\mathrm{U}$ \\
\hline $106-93-4$ & 1,2-Dibromoethane & 0.50 & $\mathrm{U}$ \\
\hline $108-90-7$ & Chlorobenzene & 0.50 & $\mathrm{U}$ \\
\hline $100-41-4$ & Ethylbenzene & 0.023 & $\mathrm{~J}$ \\
\hline $95-47-6$ & o-Xylene & 0.084 & $\mathrm{~J}$ \\
\hline $179601-23-1$ & $\mathrm{~m}, \mathrm{p}-\mathrm{Xyl}$ lene & 0.061 & $\mathrm{~J}$ \\
\hline $100-42-5$ & Styrene & 0.024 & $\mathrm{~J}$ \\
\hline $75-25-2$ & Bromoform & 0.50 & $\mathrm{U}$ \\
\hline $98-82-8$ & Isopropylbenzene & 0.50 & $\mathrm{U}$ \\
\hline $79-34-5$ & $1,1,2,2$-Tetrachloroethane & 0.50 & $\mathrm{U}$ \\
\hline $541-73-1$ & 1,3-Dichlorobenzene & 0.50 & $\mathrm{U}$ \\
\hline $106-46-7$ & 1,4-Dichlorobenzene & 0.50 & $\mathrm{U}$ \\
\hline $95-50-1$ & 1,2-Dichlorobenzene & 0.50 & $\mathrm{U}$ \\
\hline $96-12-8$ & 1,2-Dibromo-3-Chloropropane & 0.50 & $\mathrm{U}$ \\
\hline $120-82-1$ & 1,2,4-Trichlorobenzene & 0.50 & $\mathrm{U}$ \\
\hline $87-61-6$ & $1,2,3$-Trichlorobenzene & 0.50 & $\mathrm{U}$ \\
\hline
\end{tabular}


$1 \mathrm{~J}$ - FORM I VOA-TIC

VOLATILE ORGANICS ANALYSIS DATA SHEET

TENTATIVELY IDENTIFIED COMPOUNDS
EPA SAMPLE NO.

EVQCTB-W-29842

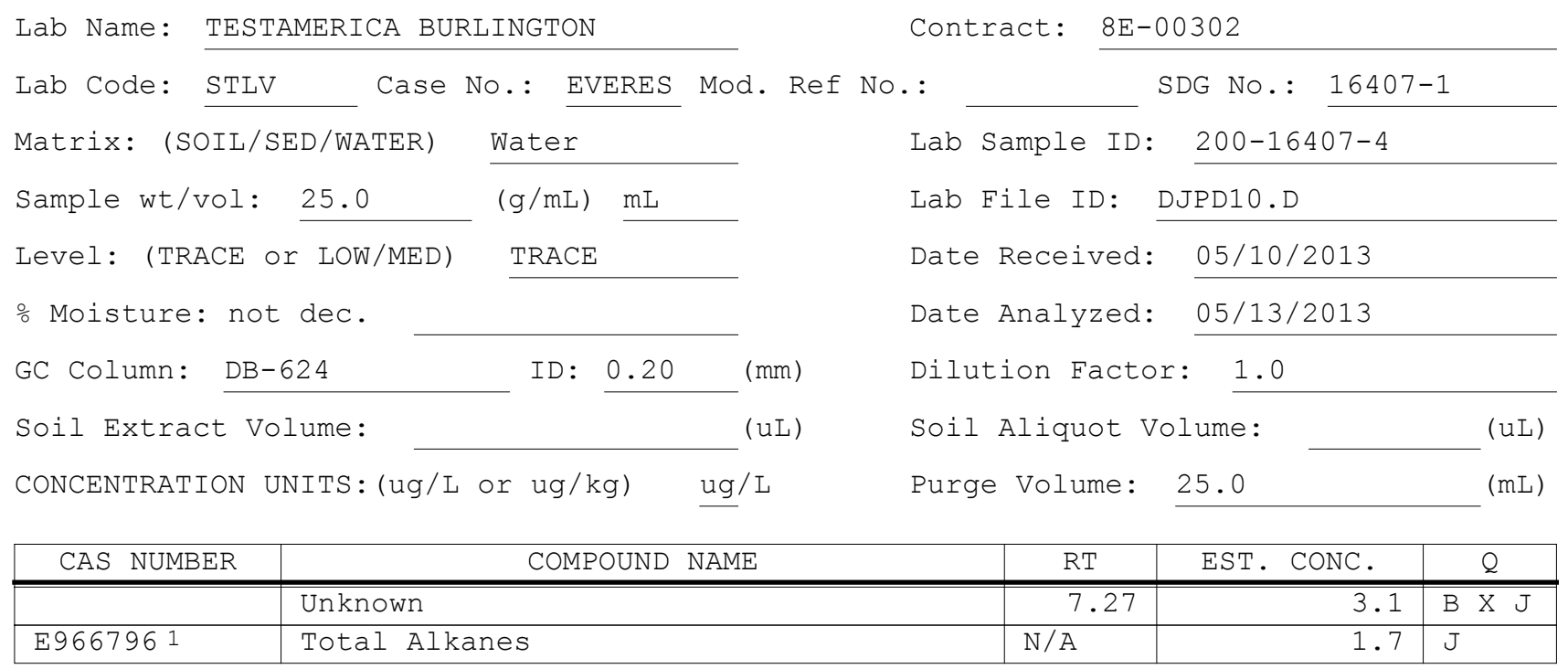

1 EPA-designated Registry Number. 
Lab Name: TESTAMERICA BURLINGTON Case No.: EVERES Mod. Ref No.:
Lab Code: STLV

Contract: 8E-00302

\section{Matrix: (SOIL/SED/WATER)}

Water

Sample wt/vol: 25.0 $(\mathrm{g} / \mathrm{mL}) \quad \mathrm{mL}$

Level: (TRACE/LOW/MED) TRACE

\% Moisture: not dec.

GC Column: DB-624 ID $: 0.20 \quad(\mathrm{~mm})$

Soil Extract Volume: (uL) $(\mathrm{mL})$

Purge Volume: 25.0

Lab File ID: DJPD09.D

Dilution Factor: 1.0

Soil Aliquot Volume: SDG No.: 16407-1 Lab Sample ID: 200-16407-3

Date Received: 05/10/2013

Date Analyzed: 05/13/2013

Purge Volume: 25.0

\begin{tabular}{|c|c|c|c|}
\hline CAS NO. & COMPOUND & $\begin{array}{l}\text { CONCENTRATION UNITS: } \\
(\mathrm{ug} / \mathrm{L} \text { or } \mathrm{ug} / \mathrm{kg}) \mathrm{ug} / \mathrm{L}\end{array}$ & Q \\
\hline $75-71-8$ & Dichlorodifluoromethane & 0.50 & $\bar{U}$ \\
\hline $74-87-3$ & Chloromethane & 0.50 & $\mathrm{U}$ \\
\hline $75-01-4$ & Vinyl chloride & 0.50 & $\mathrm{U}$ \\
\hline $74-83-9$ & Bromomethane & 0.50 & $\mathrm{U}$ \\
\hline $75-00-3$ & Chloroethane & 0.50 & $\mathrm{U}$ \\
\hline $75-69-4$ & Trichlorofluoromethane & 0.50 & $\mathrm{U}$ \\
\hline $75-35-4$ & 1,1-Dichloroethene & 0.50 & $\mathrm{U}$ \\
\hline $76-13-1$ & 1,1,2-Trichloro-1,2,2-trifluoroethane & 0.50 & $\mathrm{U}$ \\
\hline $67-64-1$ & Acetone & 5.0 & $\mathrm{U}$ \\
\hline $75-15-0$ & Carbon disulfide & 0.50 & $\mathrm{U}$ \\
\hline $79-20-9$ & Methyl acetate & 0.50 & $\mathrm{U}$ \\
\hline $75-09-2$ & Methylene Chloride & 0.50 & $\mathrm{U}$ \\
\hline $156-60-5$ & trans-1,2-Dichloroethene & 0.50 & $\mathrm{U}$ \\
\hline $1634-04-4$ & Methyl tert-butyl ether & 0.50 & $\mathrm{U}$ \\
\hline $75-34-3$ & 1,1-Dichloroethane & 0.50 & $\mathrm{U}$ \\
\hline $156-59-2$ & cis-1,2-Dichloroethene & 0.50 & $\mathrm{U}$ \\
\hline $78-93-3$ & 2-Butanone & 5.0 & $\mathrm{U}$ \\
\hline $74-97-5$ & Bromochloromethane & 0.50 & $\mathrm{U}$ \\
\hline $67-66-3$ & Chloroform & 0.50 & $\mathrm{U}$ \\
\hline $71-55-6$ & 1,1,1-Trichloroethane & 0.50 & $\mathrm{U}$ \\
\hline $110-82-7$ & Cyclohexane & 0.50 & $\mathrm{U}$ \\
\hline $56-23-5$ & Carbon tetrachloride & 0.50 & $\mathrm{U}$ \\
\hline $71-43-2$ & Benzene & 0.50 & $\mathrm{U}$ \\
\hline $107-06-2$ & 1,2-Dichloroethane & 0.50 & $\mathrm{U}$ \\
\hline
\end{tabular}

Report 1,4-Dioxane for Low-Medium VOA analysis only 
Lab Name: TESTAMERICA BURLINGTON

Lab Code: STLV Case No.: EVERES Mod. Ref No.:
Contract: 8E-00302 SDG No.: 16407-1
Matrix: (SOIL/SED/WATER)

Water

Sample wt/vol: 25.0 $(\mathrm{g} / \mathrm{mL}) \quad \mathrm{mL}$

Level : (TRACE/LOW/MED)

TRACE

\% Moisture: not dec.

GC Column: DB-624

Soil Extract Volume: ID $: 0.20 \quad(\mathrm{~mm})$

Purge Volume: 25.0
(uL)

$(\mathrm{mL})$
Lab Sample ID: 200-16407-3

Lab File ID: DJPD09.D

Date Received: 05/10/2013

Date Analyzed: 05/13/2013

Dilution Factor: 1.0

Soil Aliquot Volume: $(u L)$

\begin{tabular}{|c|c|c|c|}
\hline CAS NO. & COMPOUND & $\begin{array}{l}\text { CONCENTRATION UNITS: } \\
(\mathrm{ug} / \mathrm{L} \text { or } \mathrm{ug} / \mathrm{kg}) \mathrm{ug} / \mathrm{L}\end{array}$ & \& \\
\hline $79-01-6$ & Trichloroethene & 0.50 & $\overline{\mathrm{U}}$ \\
\hline $108-87-2$ & Methylcyclohexane & 0.50 & $\mathrm{U}$ \\
\hline $78-87-5$ & 1,2-Dichloropropane & 0.50 & $\mathrm{U}$ \\
\hline $75-27-4$ & Bromodichloromethane & 0.50 & $\mathrm{U}$ \\
\hline $10061-01-5$ & cis-1,3-Dichloropropene & 0.50 & $\mathrm{U}$ \\
\hline $108-10-1$ & 4-Methyl-2-pentanone & 5.0 & $\mathrm{U}$ \\
\hline $108-88-3$ & Toluene & 0.046 & $\mathrm{~J}$ \\
\hline $10061-02-6$ & trans-1,3-Dichloropropene & 0.50 & $\mathrm{U}$ \\
\hline $79-00-5$ & $1,1,2$-Trichloroethane & 0.50 & $\mathrm{U}$ \\
\hline $127-18-4$ & Tetrachloroethene & 0.50 & $\mathrm{U}$ \\
\hline $591-78-6$ & 2-Hexanone & 5.0 & $\mathrm{U}$ \\
\hline $124-48-1$ & Dibromochloromethane & 0.50 & $\mathrm{U}$ \\
\hline $106-93-4$ & 1,2-Dibromoethane & 0.50 & $\mathrm{U}$ \\
\hline $108-90-7$ & Chlorobenzene & 0.50 & $\mathrm{U}$ \\
\hline $100-41-4$ & Ethylbenzene & 0.0059 & $\mathrm{~J}$ \\
\hline $95-47-6$ & o-Xylene & 0.0030 & $\mathrm{~J}$ \\
\hline $179601-23-1$ & $\mathrm{~m}, \mathrm{p}-\mathrm{Xyl}$ lene & 0.027 & $\mathrm{~J}$ \\
\hline $100-42-5$ & Styrene & 0.50 & $\mathrm{U}$ \\
\hline $75-25-2$ & Bromoform & 0.50 & $\mathrm{U}$ \\
\hline $98-82-8$ & Isopropylbenzene & 0.50 & $\mathrm{U}$ \\
\hline $79-34-5$ & $1,1,2,2$-Tetrachloroethane & 0.50 & $\mathrm{U}$ \\
\hline $541-73-1$ & 1,3-Dichlorobenzene & 0.50 & $\mathrm{U}$ \\
\hline $106-46-7$ & 1,4-Dichlorobenzene & 0.50 & $\mathrm{U}$ \\
\hline $95-50-1$ & 1,2-Dichlorobenzene & 0.50 & $\mathrm{U}$ \\
\hline $96-12-8$ & 1,2-Dibromo-3-Chloropropane & 0.50 & $\mathrm{U}$ \\
\hline $120-82-1$ & 1,2,4-Trichlorobenzene & 0.50 & $\mathrm{U}$ \\
\hline $87-61-6$ & $1,2,3$-Trichlorobenzene & 0.50 & $\mathrm{U}$ \\
\hline
\end{tabular}




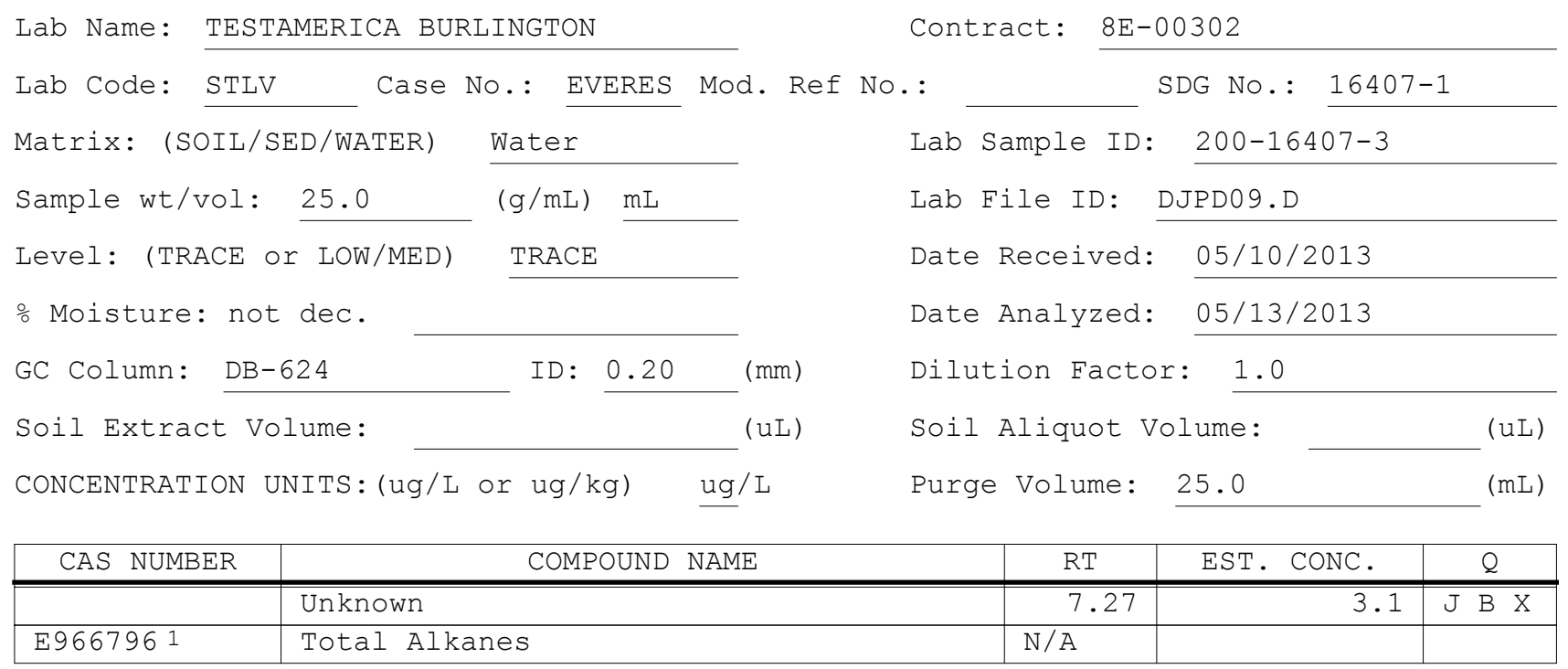


Lab Name: TESTAMERICA BURLINGTON

Contract: $\quad 8 \mathrm{E}-00302$

Lab Code: STLV

Case No.: EVERES Mod. Ref No.:

Matrix: (SOIL/SED/WATER)

Water

Sample wt/vol: 25.0 $(\mathrm{g} / \mathrm{mL}) \quad \mathrm{mL}$

Level: (TRACE/LOW/MED) TRACE

\% Moisture: not dec.

GC Column: DB-624

Soil Extract Volume: ID $: 0.20 \quad(\mathrm{~mm})$

Purge Volume: 25.0 SDG No.: 16407-1

Lab Sample ID: 200-16407-5

Lab File ID: DJPD11.D

Date Received: 05/10/2013

Date Analyzed: 05/13/2013

Dilution Factor: 1.0

Soil Aliquot Volume:

\begin{tabular}{|c|c|c|c|}
\hline CAS NO. & COMPOUND & $\begin{array}{l}\text { CONCENTRATION UNITS: } \\
(\mathrm{ug} / \mathrm{L} \text { or } \mathrm{ug} / \mathrm{kg}) \mathrm{ug} / \mathrm{L}\end{array}$ & Q \\
\hline $75-71-8$ & Dichlorodifluoromethane & 0.50 & $\overline{\mathrm{U}}$ \\
\hline $74-87-3$ & Chloromethane & 0.50 & $\mathrm{U}$ \\
\hline $75-01-4$ & Vinyl chloride & 0.50 & $\mathrm{U}$ \\
\hline $74-83-9$ & Bromomethane & 0.50 & $\mathrm{U}$ \\
\hline $75-00-3$ & Chloroethane & 0.50 & $\mathrm{U}$ \\
\hline $75-69-4$ & Trichlorofluoromethane & 0.50 & U \\
\hline $75-35-4$ & 1,1-Dichloroethene & 0.50 & $\mathrm{U}$ \\
\hline $76-13-1$ & $1,1,2$-Trichloro-1,2,2-trifluoroethane & 0.50 & $\mathrm{U}$ \\
\hline $67-64-1$ & Acetone & 5.0 & $\mathrm{U}$ \\
\hline $75-15-0$ & Carbon disulfide & 0.50 & $\mathrm{U}$ \\
\hline $79-20-9$ & Methyl acetate & 0.50 & $\mathrm{U}$ \\
\hline $75-09-2$ & Methylene Chloride & 0.50 & $\mathrm{U}$ \\
\hline $156-60-5$ & trans-1,2-Dichloroethene & 0.50 & U \\
\hline $1634-04-4$ & Methyl tert-butyl ether & 0.50 & $\mathrm{U}$ \\
\hline $75-34-3$ & 1,1-Dichloroethane & 0.50 & $\mathrm{U}$ \\
\hline $156-59-2$ & cis-1,2-Dichloroethene & 0.50 & $\mathrm{U}$ \\
\hline $78-93-3$ & 2-Butanone & 5.0 & $\mathrm{U}$ \\
\hline $74-97-5$ & Bromochloromethane & 0.50 & $\mathrm{U}$ \\
\hline $67-66-3$ & Chloroform & 0.12 & $\mathrm{~J}$ \\
\hline $71-55-6$ & $1,1,1$-Trichloroethane & 0.50 & $\mathrm{U}$ \\
\hline $110-82-7$ & Cyclohexane & 0.50 & $\mathrm{U}$ \\
\hline $56-23-5$ & Carbon tetrachloride & 2.1 & \\
\hline $71-43-2$ & Benzene & 0.50 & $\mathrm{U}$ \\
\hline $107-06-2$ & 1,2-Dichloroethane & 0.50 & $\mathrm{U}$ \\
\hline
\end{tabular}

Report 1,4-Dioxane for Low-Medium VOA analysis only 
Lab Name: TESTAMERICA BURLINGTON Case No.: EVERES Mod. Ref No.:
Lab Code: STLV

Contract: 8E-00302

\section{Matrix: (SOIL/SED/WATER)}

Water

Sample wt/vol: 25.0 $(\mathrm{g} / \mathrm{mL}) \quad \mathrm{mL}$

Level: (TRACE/LOW/MED) TRACE

\% Moisture: not dec.

GC Column: DB-624 ID $: 0.20 \quad(\mathrm{~mm})$

Soil Extract Volume: (uL) $(\mathrm{mL})$

Purge Volume: 25.0 SDG No.: 16407-1

Lab Sample ID: 200-16407-5

Lab File ID: DJPD11.D

Date Received: 05/10/2013

Date Analyzed: 05/13/2013

Dilution Factor: 1.0

Soil Aliquot Volume: (uL)

\begin{tabular}{|c|c|c|c|}
\hline CAS NO. & COMPOUND & $\begin{array}{l}\text { CONCENTRATION UNITS: } \\
(\mathrm{ug} / \mathrm{L} \text { or } \mathrm{ug} / \mathrm{kg}) \mathrm{ug} / \mathrm{L}\end{array}$ & \& \\
\hline $79-01-6$ & Trichloroethene & 0.50 & $\overline{\mathrm{U}}$ \\
\hline $108-87-2$ & Methylcyclohexane & 0.50 & $\mathrm{U}$ \\
\hline $78-87-5$ & 1,2-Dichloropropane & 0.50 & $\mathrm{U}$ \\
\hline $75-27-4$ & Bromodichloromethane & 0.50 & $\mathrm{U}$ \\
\hline $10061-01-5$ & cis-1,3-Dichloropropene & 0.50 & $\mathrm{U}$ \\
\hline $108-10-1$ & 4-Methyl-2-pentanone & 5.0 & $\mathrm{U}$ \\
\hline $108-88-3$ & Toluene & 0.042 & $\mathrm{~J}$ \\
\hline $10061-02-6$ & trans-1,3-Dichloropropene & 0.50 & $\mathrm{U}$ \\
\hline $79-00-5$ & $1,1,2$-Trichloroethane & 0.50 & $\mathrm{U}$ \\
\hline $127-18-4$ & Tetrachloroethene & 0.50 & $\mathrm{U}$ \\
\hline $591-78-6$ & 2-Hexanone & 5.0 & $\mathrm{U}$ \\
\hline $124-48-1$ & Dibromochloromethane & 0.50 & $\mathrm{U}$ \\
\hline $106-93-4$ & 1,2-Dibromoethane & 0.50 & $\mathrm{U}$ \\
\hline $108-90-7$ & Chlorobenzene & 0.50 & $\mathrm{U}$ \\
\hline $100-41-4$ & Ethylbenzene & 0.50 & $\mathrm{U}$ \\
\hline $95-47-6$ & o-Xylene & 0.0051 & $\mathrm{~J}$ \\
\hline $179601-23-1$ & $\mathrm{~m}, \mathrm{p}-\mathrm{Xyl}$ lene & 0.026 & $\mathrm{~J}$ \\
\hline $100-42-5$ & Styrene & 0.50 & $\mathrm{U}$ \\
\hline $75-25-2$ & Bromoform & 0.50 & $\mathrm{U}$ \\
\hline $98-82-8$ & Isopropylbenzene & 0.50 & $\mathrm{U}$ \\
\hline $79-34-5$ & $1,1,2,2$-Tetrachloroethane & 0.50 & $\mathrm{U}$ \\
\hline $541-73-1$ & 1,3-Dichlorobenzene & 0.50 & $\mathrm{U}$ \\
\hline $106-46-7$ & 1,4-Dichlorobenzene & 0.50 & $\mathrm{U}$ \\
\hline $95-50-1$ & 1,2-Dichlorobenzene & 0.50 & $\mathrm{U}$ \\
\hline $96-12-8$ & 1,2-Dibromo-3-Chloropropane & 0.50 & $\mathrm{U}$ \\
\hline $120-82-1$ & 1,2,4-Trichlorobenzene & 0.50 & $\mathrm{U}$ \\
\hline $87-61-6$ & $1,2,3$-Trichlorobenzene & 0.50 & $\mathrm{U}$ \\
\hline
\end{tabular}




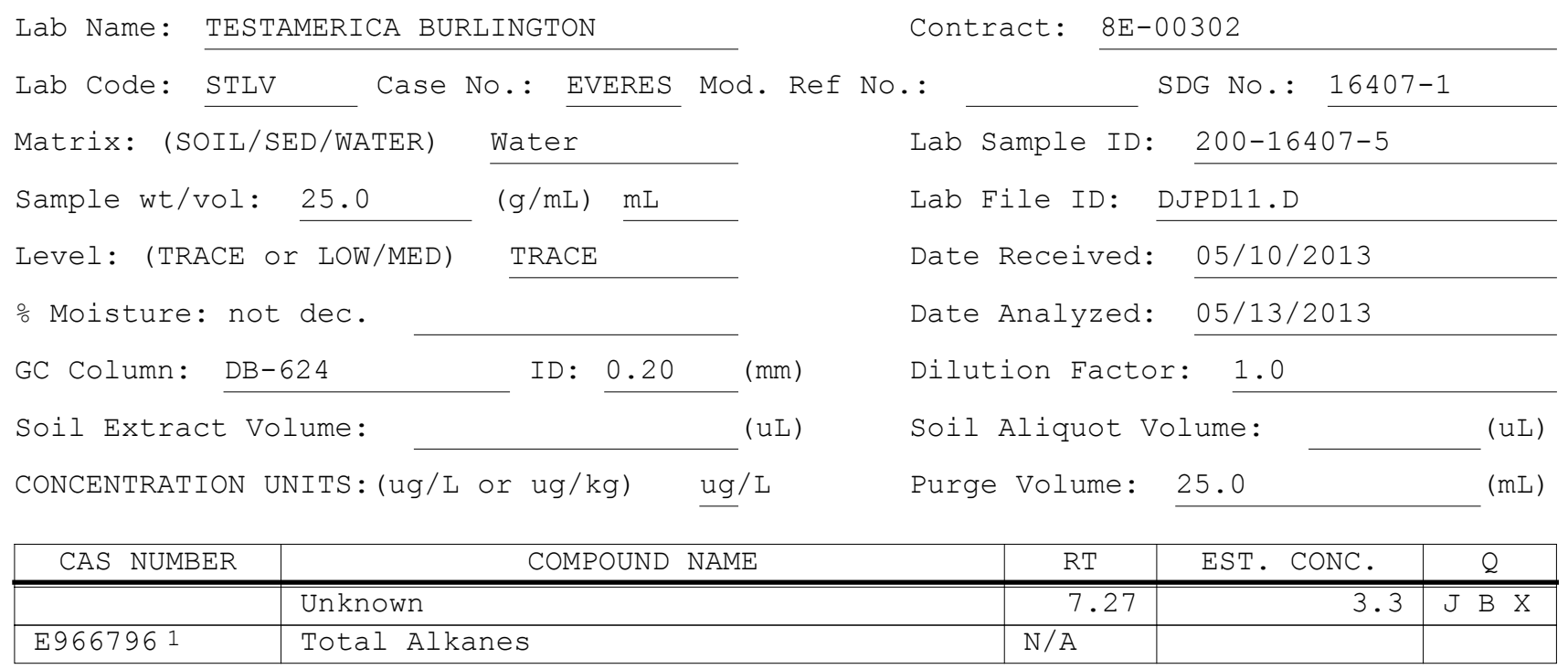


Lab Name: TESTAMERICA BURLINGTON

Lab Code: STLV Case No.: EVERES Mod. Ref No.:

Instrument ID: D.i

Heated Purge: ( $\mathrm{Y} / \mathrm{N}) \mathrm{N}$

Purge Volume: 25.0

GC Column: DB-624
Calibration Date(s):

Calibration Time(s):

(mL)

ID $: 0.20$
Contract: $8 \mathrm{E}-00302$

SDG No.: 16407-1

$05 / 01 / 201305 / 01 / 2013$

1132 1306

(m)

\begin{tabular}{|c|c|c|c|c|c|c|c|}
\hline \multirow{3}{*}{$\begin{array}{l}\text { LAB FILE ID: } \\
\text { RRF } \underline{5.0}=\text { DJP05.D } \\
\text { COMPOUND }\end{array}$} & \multirow{2}{*}{$\begin{array}{l}\text { RRF } 0.5 \\
\operatorname{RRF} 10\end{array}$} & \multirow{2}{*}{\multicolumn{2}{|c|}{$\begin{array}{l}=\mathrm{DJP03.D} \\
=\mathrm{DJP} 06 . \mathrm{D}\end{array}$}} & \multirow{2}{*}{$\begin{array}{l}\text { RRF } 1.0 \\
\text { RRF } 20\end{array}$} & \multirow{2}{*}{\multicolumn{3}{|c|}{$\begin{array}{l}=\text { DJP04.D } \\
=\text { DJP07.D }\end{array}$}} \\
\hline & & & & & & & \\
\hline & $\mathrm{RRF} 0.5$ & RRE 1.0 & RRF 5.0 & RRF 10 & RRF 20 & $\overline{\mathrm{RRF}}$ & $\because \mathrm{RSD}$ \\
\hline Dichlorodifluoromethane & 0.563 & 0.547 & 0.521 & 0.520 & 0.544 & 0.539 & 3.4 \\
\hline Chloromethane & 0.560 & 0.559 & 0.520 & 0.532 & 0.549 & 0.544 & 3.2 \\
\hline Vinyl chloride & 0.560 & 0.561 & 0.548 & 0.561 & 0.579 & 0.562 & 2.0 \\
\hline Bromomethane & 0.291 & 0.289 & 0.288 & 0.318 & 0.337 & 0.305 & 7.3 \\
\hline Chloroethane & 0.332 & 0.334 & 0.324 & 0.324 & 0.325 & 0.328 & 1.5 \\
\hline Trichlorofluoromethane & 0.830 & 0.822 & 0.810 & 0.804 & 0.865 & 0.826 & 2.9 \\
\hline 1,1-Dichloroethene & 0.283 & 0.280 & 0.286 & 0.299 & 0.331 & 0.296 & 7.1 \\
\hline $\begin{array}{l}1,1,2 \text {-Trichloro- } \\
1,2,2 \text {-trifluoroethane }\end{array}$ & 0.340 & 0.350 & 0.345 & 0.347 & 0.386 & 0.353 & 5.2 \\
\hline Acetone & 0.021 & 0.021 & 0.019 & 0.019 & 0.020 & 0.020 & 5.8 \\
\hline Carbon disulfide & 0.666 & 0.681 & 0.712 & 0.730 & 0.773 & 0.712 & 5.9 \\
\hline Methyl acetate & 0.047 & 0.049 & 0.049 & 0.050 & 0.048 & 0.049 & 2.5 \\
\hline Methylene Chloride & 0.285 & 0.269 & 0.245 & 0.246 & 0.256 & 0.260 & 6.5 \\
\hline trans-1,2-Dichloroethene & 0.299 & 0.306 & 0.305 & 0.314 & 0.337 & 0.312 & 4.8 \\
\hline Methyl tert-butyl ether & 0.345 & 0.360 & 0.370 & 0.377 & 0.415 & 0.374 & 7.0 \\
\hline 1,1-Dichloroethane & 0.533 & 0.540 & 0.534 & 0.540 & 0.571 & 0.544 & 2.9 \\
\hline cis-1,2-Dichloroethene & 0.303 & 0.308 & 0.306 & 0.314 & 0.333 & 0.313 & 3.8 \\
\hline 2-Butanone & 0.027 & 0.029 & 0.030 & 0.030 & 0.033 & 0.030 & 7.1 \\
\hline Bromochloromethane & 0.090 & 0.093 & 0.095 & 0.096 & 0.104 & 0.096 & 5.2 \\
\hline Chloroform & 0.512 & 0.530 & 0.527 & 0.534 & 0.564 & 0.533 & 3.6 \\
\hline $1,1,1$-Trichloroethane & 0.592 & 0.587 & 0.597 & 0.617 & 0.639 & 0.606 & 3.5 \\
\hline Cyclohexane & 0.662 & 0.673 & 0.697 & 0.698 & 0.730 & 0.692 & 3.8 \\
\hline Carbon tetrachloride & 0.512 & 0.514 & 0.538 & 0.555 & 0.589 & 0.542 & 5.9 \\
\hline Benzene & 1.567 & 1.581 & 1.555 & 1.576 & 1.659 & 1.588 & 2.6 \\
\hline 1,2-Dichloroethane & 0.247 & 0.257 & 0.256 & 0.260 & 0.287 & 0.261 & 5.7 \\
\hline Trichloroethene & 0.404 & 0.402 & 0.394 & 0.404 & 0.416 & 0.404 & 1.9 \\
\hline Methylcyclohexane & 0.536 & 0.550 & 0.575 & 0.566 & 0.615 & 0.568 & 5.3 \\
\hline
\end{tabular}

Report 1,4-Dioxane for Low-Medium VOA analysis only 
Lab Name: TESTAMERICA BURLINGTON

Lab Code: STLV Case No.: EVERES Mod. Ref No.:

Instrument ID: D.i

Heated Purge: ( $\mathrm{Y} / \mathrm{N}) \mathrm{N}$

Purge Volume: 25.0

GC Column: DB-624
Calibration Date (s):

Calibration Time(s):

(mL)

ID $: 0.20$
Contract: $8 \mathrm{E}-00302$

SDG No.: 16407-1

05/01/201305/01/2013

1132 1306

(m)

\begin{tabular}{|c|c|c|c|c|c|c|c|}
\hline \multirow{2}{*}{$\begin{array}{l}\text { LAB FILE ID: } \\
\text { RRF } 5.0=\text { DJP05.D }\end{array}$} & \multicolumn{3}{|c|}{ RRF0.5 = DJP03.D } & \multicolumn{4}{|c|}{ RRF1.0 = DJP04.D } \\
\hline & RRF 10 & P06.D & & RRE 20 & $=\mathrm{DJP}$ & . D & \\
\hline COMPOUND & $\mathrm{RRF} 0.5$ & RRF 1.0 & RRF 5.0 & RRF 10 & RRE 20 & $\overline{\mathrm{RRF}}$ & $\because \mathrm{RSD}$ \\
\hline 1,2-Dichloropropane & 0.318 & 0.323 & 0.322 & 0.329 & 0.349 & 0.328 & 3.7 \\
\hline Bromodichloromethane & 0.313 & 0.319 & 0.341 & 0.353 & 0.370 & 0.339 & 6.9 \\
\hline cis-1,3-Dichloropropene & 0.388 & 0.409 & 0.451 & 0.460 & 0.479 & 0.437 & 8.6 \\
\hline 4-Methyl-2-pentanone & 0.075 & 0.082 & 0.088 & 0.090 & 0.099 & 0.087 & 10.0 \\
\hline Toluene & 1.661 & 1.688 & 1.726 & 1.819 & 1.963 & 1.771 & 6.9 \\
\hline trans-1,3-Dichloropropene & 0.252 & 0.292 & 0.322 & 0.337 & 0.361 & 0.313 & 13.5 \\
\hline 1,1,2-Trichloroethane & 0.150 & 0.155 & 0.152 & 0.155 & 0.161 & 0.155 & 2.8 \\
\hline Tetrachloroethene & 0.325 & 0.327 & 0.326 & 0.337 & 0.367 & 0.336 & 5.4 \\
\hline 2-Hexanone & 0.049 & 0.055 & 0.059 & 0.061 & 0.067 & 0.058 & 11.8 \\
\hline Dibromochloromethane & 0.143 & 0.155 & 0.174 & 0.183 & 0.200 & 0.171 & 13.1 \\
\hline 1,2-Dibromoethane & 0.132 & 0.137 & 0.138 & 0.142 & 0.150 & 0.140 & 4.6 \\
\hline Chlorobenzene & 1.027 & 1.045 & 1.023 & 1.057 & 1.142 & 1.059 & 4.6 \\
\hline Ethylbenzene & 1.847 & 1.904 & 1.982 & 2.132 & 2.307 & 2.034 & 9.2 \\
\hline o-Xylene & 0.632 & 0.674 & 0.728 & 0.797 & 0.892 & 0.744 & 13.9 \\
\hline $\mathrm{m}, \mathrm{p}-\mathrm{xylene}$ & 0.671 & 0.713 & 0.774 & 0.827 & 0.904 & 0.778 & 11.9 \\
\hline Styrene & 0.772 & 0.868 & 1.050 & 1.195 & 1.348 & 1.046 & 22.4 \\
\hline Bromoform & 0.103 & 0.119 & 0.136 & 0.149 & 0.172 & 0.136 & 19.5 \\
\hline Isopropylbenzene & 1.751 & 1.851 & 2.016 & 2.198 & 2.402 & 2.044 & 12.8 \\
\hline $1,1,2,2$-Tetrachloroethane & 0.120 & 0.136 & 0.138 & 0.144 & 0.153 & 0.138 & 8.7 \\
\hline 1,3-Dichlorobenzene & 1.515 & 1.554 & 1.565 & 1.635 & 1.770 & 1.608 & 6.3 \\
\hline 1,4-Dichlorobenzene & 1.586 & 1.587 & 1.536 & 1.595 & 1.705 & 1.602 & 3.9 \\
\hline 1,2-Dichlorobenzene & 1.196 & 1.249 & 1.241 & 1.296 & 1.405 & 1.277 & 6.2 \\
\hline 1,2-Dibromo-3-Chloropropane & 0.024 & 0.030 & 0.034 & 0.036 & 0.039 & 0.032 & 18.2 \\
\hline 1,2,4-Trichlorobenzene & 0.632 & 0.652 & 0.698 & 0.712 & 0.796 & 0.698 & 9.1 \\
\hline 1,2,3-Trichlorobenzene & 0.375 & 0.407 & 0.418 & 0.426 & 0.456 & 0.416 & 7.0 \\
\hline
\end{tabular}




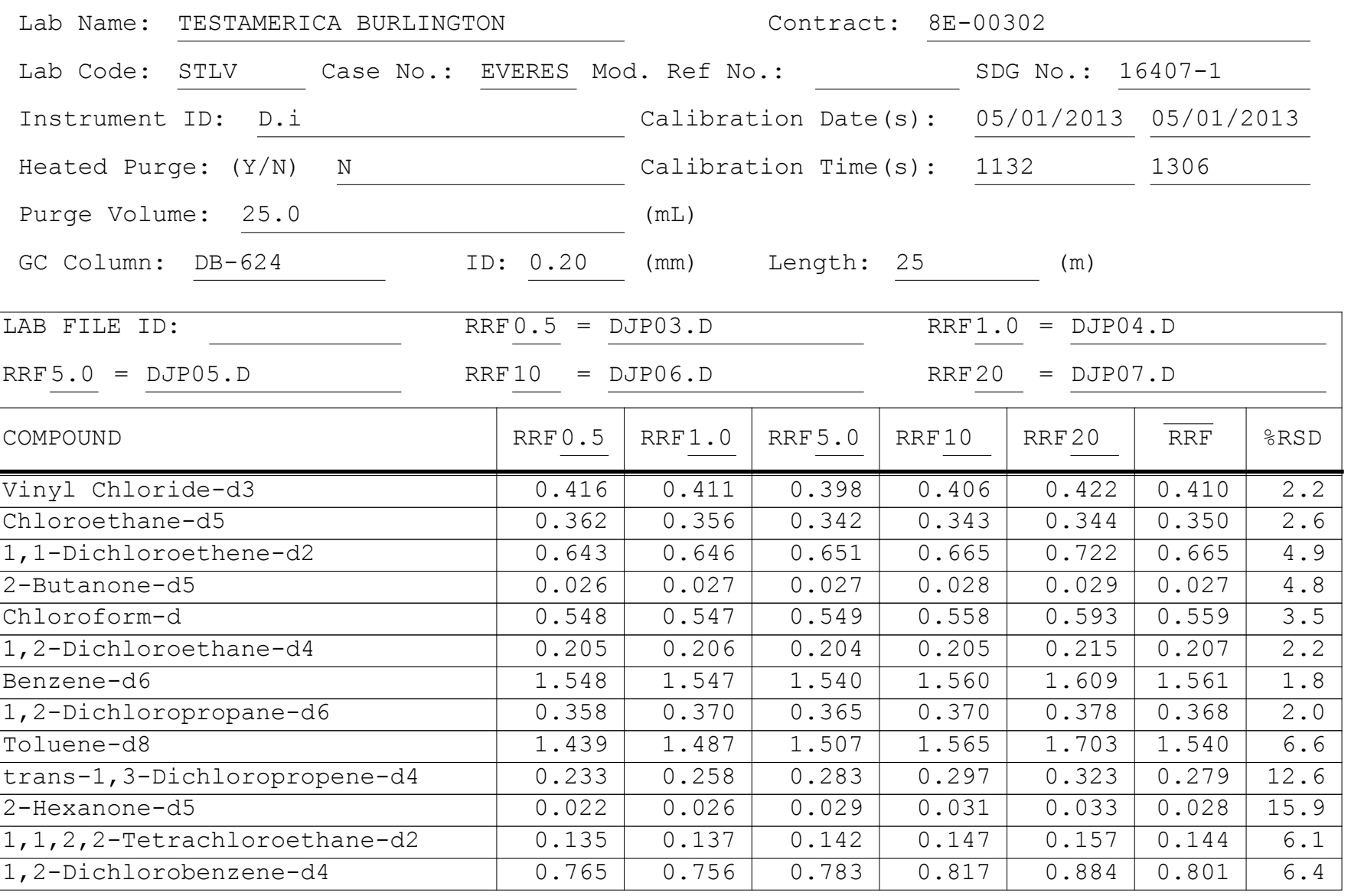

Report 1,4-Dioxane-d8 for Low-Medium VOA analysis only 
Lab Name: TESTAMERICA BURLINGTON

Lab Code: STLV Case No.: EVERES Mod. Ref No.:

Instrument ID: D.i

Lab File Id: DJPD02.D

EPA Sample No. (VSTD\#\#\#\#) :

Heated Purge: ( $\mathrm{Y} / \mathrm{N}) \mathrm{N}$

Purge Volume: 25.0
Contract: 8E-00302

Calibration Date: 05/13/2013 Time: 1033 Init. Calib. Date(s): 05/01/2013 05/01/2013

Init. Calib. Time(s): $1132 \quad 1306$ ID: $0.20(\mathrm{~mm})$ Length: $25 \quad(\mathrm{~m})$

\begin{tabular}{|c|c|c|c|c|c|}
\hline COMPOUND & $\overline{\mathrm{RRF}}$ & RRF5. 0 & $\begin{array}{l}\text { MIN } \\
\text { RRF }\end{array}$ & $\div D$ & $\mathrm{MAX} \div \mathrm{D}$ \\
\hline Dichlorodifluoromethane & 0.539 & 0.580 & 0.010 & 7.6 & 40.0 \\
\hline Chloromethane & 0.544 & 0.573 & 0.010 & 5.4 & 40.0 \\
\hline Vinyl chloride & 0.562 & 0.608 & 0.100 & 8.2 & 30.0 \\
\hline Bromomethane & 0.305 & 0.314 & 0.100 & 3.1 & 30.0 \\
\hline Chloroethane & 0.328 & 0.340 & 0.010 & 3.8 & 40.0 \\
\hline Trichlorofluoromethane & 0.826 & 0.876 & 0.010 & 6.1 & 40.0 \\
\hline 1,1-Dichloroethene & 0.296 & 0.309 & 0.100 & 4.5 & 30.0 \\
\hline 1,1,2-Trichloro-1,2,2-trifluoroethane & 0.353 & 0.372 & 0.010 & 5.3 & 40.0 \\
\hline Acetone & 0.020 & 0.019 & 0.010 & -3.6 & 40.0 \\
\hline Carbon disulfide & 0.712 & 0.788 & 0.010 & 10.7 & 40.0 \\
\hline Methyl acetate & 0.049 & 0.048 & 0.010 & -1.9 & 40.0 \\
\hline Methylene Chloride & 0.260 & 0.250 & 0.010 & -3.7 & 40.0 \\
\hline trans-1,2-Dichloroethene & 0.312 & 0.324 & 0.010 & 3.6 & 40.0 \\
\hline Methyl tert-butyl ether & 0.374 & 0.362 & 0.010 & -3.1 & 40.0 \\
\hline 1,1-Dichloroethane & 0.544 & 0.561 & 0.200 & 3.2 & 30.0 \\
\hline cis-1,2-Dichloroethene & 0.313 & 0.318 & 0.010 & 1.8 & 40.0 \\
\hline 2-Butanone & 0.030 & 0.030 & 0.010 & -0.3 & 40.0 \\
\hline Bromochloromethane & 0.096 & 0.093 & 0.050 & -2.4 & 30.0 \\
\hline Chloroform & 0.533 & 0.543 & 0.200 & 1.8 & 30.0 \\
\hline $1,1,1$-Trichloroethane & 0.606 & 0.650 & 0.100 & 7.2 & 30.0 \\
\hline Cyclohexane & 0.692 & 0.768 & 0.010 & 11.0 & 40.0 \\
\hline Carbon tetrachloride & 0.542 & 0.595 & 0.100 & 9.8 & 30.0 \\
\hline Benzene & 1.588 & 1.633 & 0.400 & 2.9 & 30.0 \\
\hline 1,2-Dichloroethane & 0.261 & 0.258 & 0.100 & -1.4 & 30.0 \\
\hline Trichloroethene & 0.404 & 0.419 & 0.300 & 3.8 & 30.0 \\
\hline Methylcyclohexane & 0.568 & 0.640 & 0.010 & 12.5 & 40.0 \\
\hline
\end{tabular}

Report 1,4-Dioxane for Low/Medium VOA analysis only 
Lab Name: TESTAMERICA BURLINGTON

Lab Code: STLV Case No.: EVERES Mod. Ref No.:

Instrument ID: D.i

Lab File Id: DJPD02.D

EPA Sample No.(VSTD\#\#\#\#) :

Heated Purge: ( $\mathrm{Y} / \mathrm{N}) \mathrm{N}$

Purge Volume: 25.0
Contract: $8 \mathrm{E}-00302$

Calibration Date: 05/13/2013 Time: 1033 Init. Calib. Date(s): 05/01/2013 05/01/2013

Init. Calib. Time(s) : $1132 \quad 1306$ ID: $0.20(\mathrm{~mm})$ Length: $25 \quad(\mathrm{~m})$

\begin{tabular}{|c|c|c|c|c|c|}
\hline COMPOUND & $\overline{\mathrm{RRF}}$ & RRF5. 0 & $\begin{array}{l}\text { MIN } \\
\text { RRF }\end{array}$ & $\div D$ & $\mathrm{MAX} \div \mathrm{D}$ \\
\hline 1,2-Dichloropropane & 0.328 & 0.329 & 0.010 & 0.3 & 40.0 \\
\hline Bromodichloromethane & 0.339 & 0.344 & 0.200 & 1.5 & 30.0 \\
\hline cis-1,3-Dichloropropene & 0.437 & 0.453 & 0.200 & 3.4 & 30.0 \\
\hline 4-Methyl-2-pentanone & 0.087 & 0.087 & 0.010 & -0.3 & 40.0 \\
\hline Toluene & 1.771 & 1.764 & 0.400 & -0.4 & 30.0 \\
\hline trans-1,3-Dichloropropene & 0.313 & 0.330 & 0.100 & 5.4 & 30.0 \\
\hline 1,1,2-Trichloroethane & 0.155 & 0.151 & 0.100 & -2.4 & 30.0 \\
\hline Tetrachloroethene & 0.336 & 0.347 & 0.100 & 3.1 & 30.0 \\
\hline 2-Hexanone & 0.058 & 0.059 & 0.010 & 1.5 & 40.0 \\
\hline Dibromochloromethane & 0.171 & 0.166 & 0.100 & -3.0 & 30.0 \\
\hline 1,2-Dibromoethane & 0.140 & 0.136 & 0.010 & -2.9 & 40.0 \\
\hline Chlorobenzene & 1.059 & 1.052 & 0.500 & -0.6 & 30.0 \\
\hline Ethylbenzene & 2.034 & 2.069 & 0.100 & 1.7 & 30.0 \\
\hline o-Xylene & 0.744 & 0.702 & 0.300 & -5.7 & 30.0 \\
\hline $\mathrm{m}, \mathrm{p}$-Xylene & 0.778 & 0.750 & 0.300 & -3.6 & 30.0 \\
\hline Styrene & 1.046 & 0.837 & 0.300 & -20.0 & 30.0 \\
\hline Bromoform & 0.136 & 0.131 & 0.050 & -3.5 & 30.0 \\
\hline Isopropylbenzene & 2.044 & 2.128 & 0.010 & 4.1 & 40.0 \\
\hline $1,1,2,2$-Tetrachloroethane & 0.138 & 0.137 & 0.100 & -1.0 & 30.0 \\
\hline 1,3-Dichlorobenzene & 1.608 & 1.599 & 0.400 & -0.6 & 30.0 \\
\hline 1,4-Dichlorobenzene & 1.602 & 1.576 & 0.400 & -1.6 & 30.0 \\
\hline 1,2 -Dichlorobenzene & 1.277 & 1.252 & 0.400 & -2.0 & 30.0 \\
\hline 1,2-Dibromo-3-Chloropropane & 0.032 & 0.034 & 0.010 & 4.1 & 40.0 \\
\hline $1,2,4$-Trichlorobenzene & 0.698 & 0.688 & 0.200 & -1.5 & 30.0 \\
\hline 1,2,3-Trichlorobenzene & 0.416 & 0.409 & 0.200 & -1.8 & 30.0 \\
\hline
\end{tabular}




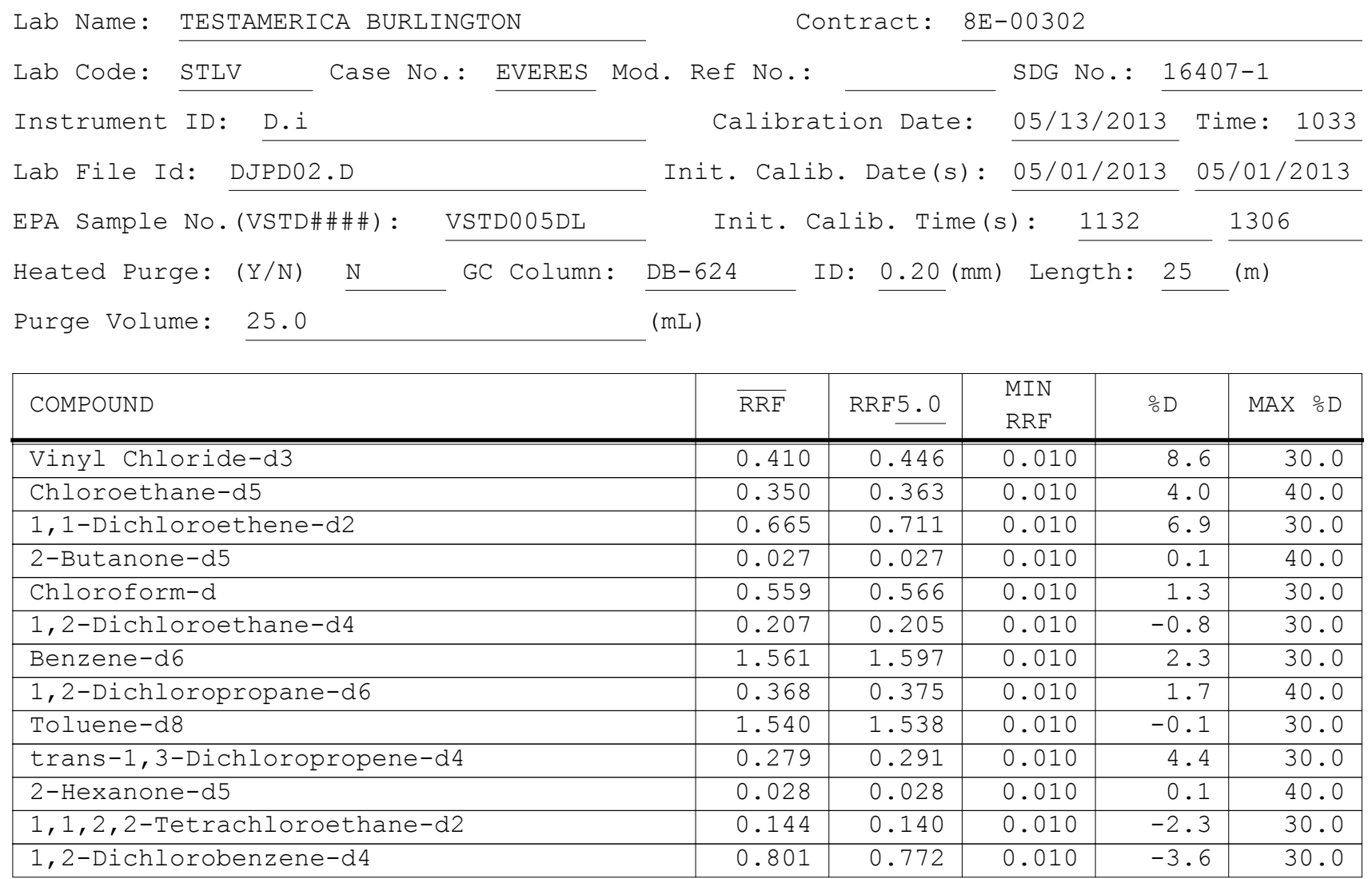


Lab Name: TESTAMERICA BURLINGTON

Lab Code: STLV Case No.: EVERES Mod. Ref No.:

Instrument ID: D.i

Lab File Id: DJPD13.D

EPA Sample No.(VSTD\#\#\#\#) :

Heated Purge: ( $\mathrm{Y} / \mathrm{N}) \mathrm{N}$

Purge Volume: 25.0
Contract: 8E-00302

Calibration Date: 05/13/2013 Time: 1518 Init. Calib. Date(s): 05/01/2013 05/01/2013

Init. Calib. Time(s): $1132 \quad 1306$ ID: $0.20(\mathrm{~mm})$ Length: $25 \quad(\mathrm{~m})$

\begin{tabular}{|c|c|c|c|c|c|}
\hline COMPOUND & $\overline{\mathrm{RRF}}$ & $\mathrm{RRF} 5.0$ & $\begin{array}{l}\text { MIN } \\
\text { RRF }\end{array}$ & $\div D$ & $\mathrm{MAX} \div \mathrm{D}$ \\
\hline Dichlorodifluoromethane & 0.539 & 0.553 & 0.010 & 2.6 & 50.0 \\
\hline Chloromethane & 0.544 & 0.559 & 0.010 & 2.8 & 50.0 \\
\hline Vinyl chloride & 0.562 & 0.580 & 0.010 & 3.3 & 50.0 \\
\hline Bromomethane & 0.305 & 0.312 & 0.010 & 2.3 & 50.0 \\
\hline Chloroethane & 0.328 & 0.334 & 0.010 & 2.0 & 50.0 \\
\hline Trichlorofluoromethane & 0.826 & 0.860 & 0.010 & 4.1 & 50.0 \\
\hline 1,1-Dichloroethene & 0.296 & 0.299 & 0.010 & 0.9 & 50.0 \\
\hline 1,1,2-Trichloro-1,2,2-trifluoroethane & 0.353 & 0.363 & 0.010 & 2.7 & 50.0 \\
\hline Acetone & 0.020 & 0.017 & 0.010 & -14.7 & 50.0 \\
\hline Carbon disulfide & 0.712 & 0.735 & 0.010 & 3.2 & 50.0 \\
\hline Methyl acetate & 0.049 & 0.050 & 0.010 & 2.5 & 50.0 \\
\hline Methylene Chloride & 0.260 & 0.250 & 0.010 & -3.7 & 50.0 \\
\hline trans-1,2-Dichloroethene & 0.312 & 0.319 & 0.010 & 2.1 & 50.0 \\
\hline Methyl tert-butyl ether & 0.374 & 0.363 & 0.010 & -2.8 & 50.0 \\
\hline 1,1-Dichloroethane & 0.544 & 0.560 & 0.010 & 3.0 & 50.0 \\
\hline cis-1,2-Dichloroethene & 0.313 & 0.313 & 0.010 & 0.1 & 50.0 \\
\hline 2-Butanone & 0.030 & 0.028 & 0.010 & -5.4 & 50.0 \\
\hline Bromochloromethane & 0.096 & 0.094 & 0.010 & -1.7 & 50.0 \\
\hline Chloroform & 0.533 & 0.547 & 0.010 & 2.4 & 50.0 \\
\hline $1,1,1$-Trichloroethane & 0.606 & 0.615 & 0.010 & 1.5 & 50.0 \\
\hline Cyclohexane & 0.692 & 0.730 & 0.010 & 5.4 & 50.0 \\
\hline Carbon tetrachloride & 0.542 & 0.562 & 0.010 & 3.7 & 50.0 \\
\hline Benzene & 1.588 & 1.587 & 0.010 & 0.0 & 50.0 \\
\hline 1,2-Dichloroethane & 0.261 & 0.265 & 0.010 & 1.5 & 50.0 \\
\hline Trichloroethene & 0.404 & 0.401 & 0.010 & -0.7 & 50.0 \\
\hline Methylcyclohexane & 0.568 & 0.617 & 0.010 & 8.5 & 50.0 \\
\hline
\end{tabular}

Report 1,4-Dioxane for Low/Medium VOA analysis only 
Lab Name: TESTAMERICA BURLINGTON

Lab Code: STLV Case No.: EVERES Mod. Ref No.:

Instrument ID: D.i

Lab File Id: DJPD13.D

EPA Sample No.(VSTD\#\#\#\#) :

Heated Purge: ( $\mathrm{Y} / \mathrm{N}) \mathrm{N}$

Purge Volume: 25.0
Contract: $8 \mathrm{E}-00302$

Calibration Date: 05/13/2013 Time: 1518 Init. Calib. Date(s): 05/01/2013 05/01/2013

Init. Calib. Time(s) : $1132 \quad 1306$ ID: $0.20(\mathrm{~mm})$ Length: $25 \quad(\mathrm{~m})$

\begin{tabular}{|c|c|c|c|c|c|}
\hline COMPOUND & $\overline{\mathrm{RRF}}$ & RRF5. 0 & $\begin{array}{l}\text { MIN } \\
\text { RRF }\end{array}$ & $\circ \mathrm{D}$ & $\mathrm{MAX} \div \mathrm{D}$ \\
\hline 1,2-Dichloropropane & 0.328 & 0.325 & 0.010 & -0.9 & 50.0 \\
\hline Bromodichloromethane & 0.339 & 0.343 & 0.010 & 1.2 & 50.0 \\
\hline cis-1,3-Dichloropropene & 0.437 & 0.449 & 0.010 & 2.6 & 50.0 \\
\hline 4-Methyl-2-pentanone & 0.087 & 0.087 & 0.010 & 0.1 & 50.0 \\
\hline Toluene & 1.771 & 1.802 & 0.010 & 1.8 & 50.0 \\
\hline trans-1,3-Dichloropropene & 0.313 & 0.325 & 0.010 & 3.8 & 50.0 \\
\hline 1,1,2-Trichloroethane & 0.155 & 0.152 & 0.010 & -1.6 & 50.0 \\
\hline Tetrachloroethene & 0.336 & 0.329 & 0.010 & -2.1 & 50.0 \\
\hline 2-Hexanone & 0.058 & 0.058 & 0.010 & 0.4 & 50.0 \\
\hline Dibromochloromethane & 0.171 & 0.169 & 0.010 & -1.0 & 50.0 \\
\hline 1,2-Dibromoethane & 0.140 & 0.135 & 0.010 & -3.7 & 50.0 \\
\hline Chlorobenzene & 1.059 & 1.043 & 0.010 & -1.5 & 50.0 \\
\hline Ethylbenzene & 2.034 & 2.097 & 0.010 & 3.1 & 50.0 \\
\hline o-Xylene & 0.744 & 0.751 & 0.010 & 0.9 & 50.0 \\
\hline $\mathrm{m}, \mathrm{p}$-Xylene & 0.778 & 0.805 & 0.010 & 3.5 & 50.0 \\
\hline Styrene & 1.046 & 1.089 & 0.010 & 4.1 & 50.0 \\
\hline Bromoform & 0.136 & 0.132 & 0.010 & -3.1 & 50.0 \\
\hline Isopropylbenzene & 2.044 & 2.133 & 0.010 & 4.4 & 50.0 \\
\hline $1,1,2,2$-Tetrachloroethane & 0.138 & 0.142 & 0.010 & 2.9 & 50.0 \\
\hline 1,3-Dichlorobenzene & 1.608 & 1.580 & 0.010 & -1.7 & 50.0 \\
\hline 1,4-Dichlorobenzene & 1.602 & 1.574 & 0.010 & -1.7 & 50.0 \\
\hline 1,2 -Dichlorobenzene & 1.277 & 1.267 & 0.010 & -0.8 & 50.0 \\
\hline 1,2-Dibromo-3-Chloropropane & 0.032 & 0.032 & 0.010 & -3.0 & 50.0 \\
\hline $1,2,4$-Trichlorobenzene & 0.698 & 0.674 & 0.010 & -3.4 & 50.0 \\
\hline 1,2,3-Trichlorobenzene & 0.416 & 0.395 & 0.010 & -5.1 & 50.0 \\
\hline
\end{tabular}




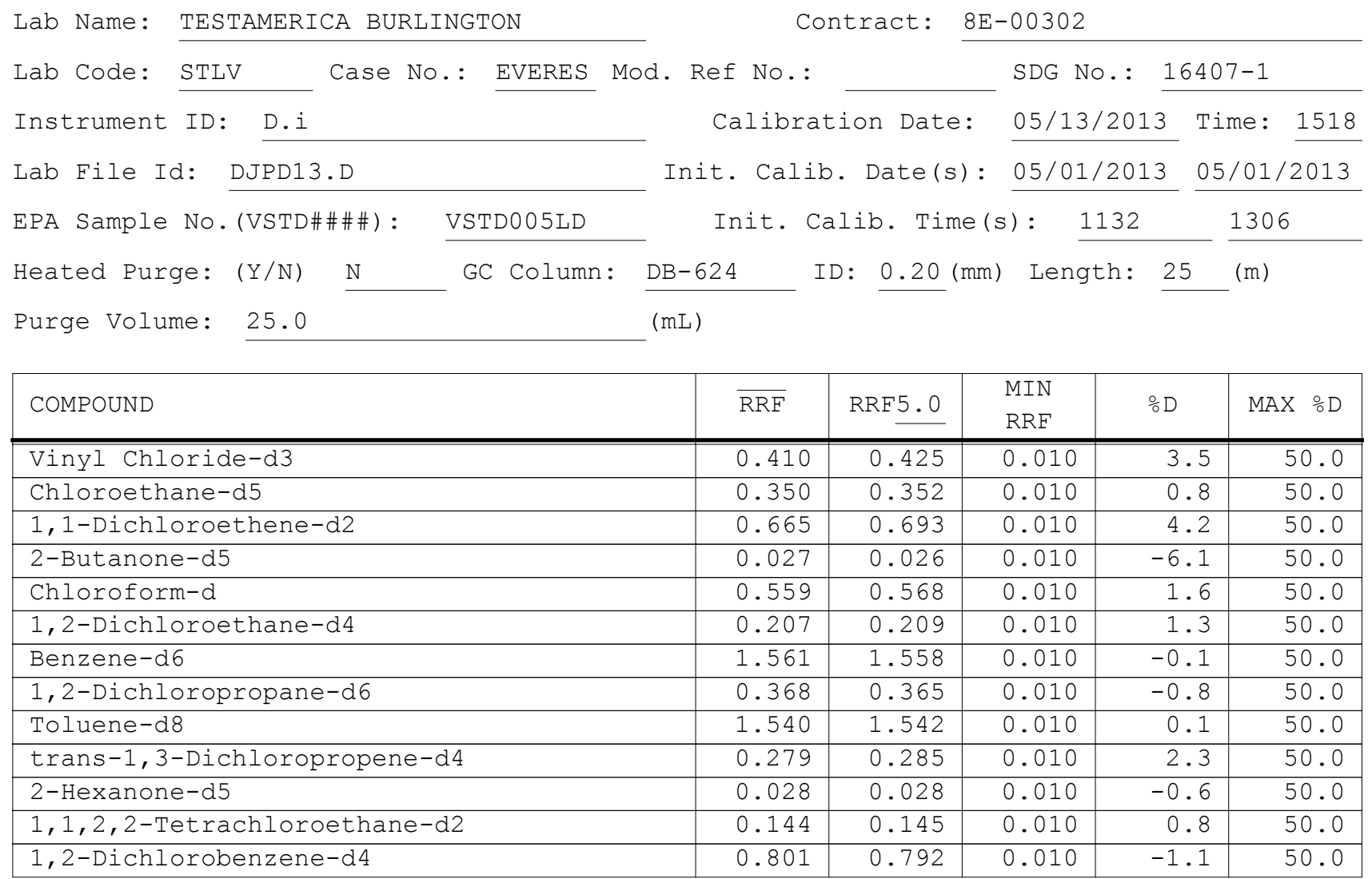


Lab Name: TESTAMERICA BURLINGTON

Contract: $\quad 8 \mathrm{E}-00302$

Lab Code: STLV Case No.: EVERES Mod. Ref No.: SDG No.: 16407-1

Matrix: (SOIL/SED/WATER)

Water

Sample wt/vol: 25.0 $(\mathrm{g} / \mathrm{mL}) \quad \mathrm{mL}$

Level: (TRACE/LOW/MED)

TRACE

\% Moisture: not dec.

GC Column: DB-624

Soil Extract Volume: ID $: 0.20 \quad(\mathrm{~mm})$

Purge Volume: 25.0
Lab Sample ID: MB 200-55472/4

Lab File ID: DJPD04.D

Date Received:

Date Analyzed: 05/13/2013

Dilution Factor: 1.0

Soil Aliquot Volume: $(u L)$

\begin{tabular}{|c|c|c|c|}
\hline CAS NO. & COMPOUND & $\begin{array}{l}\text { CONCENTRATION UNITS: } \\
(\mathrm{ug} / \mathrm{L} \text { or } \mathrm{ug} / \mathrm{kg}) \mathrm{ug} / \mathrm{L}\end{array}$ & Q \\
\hline $75-71-8$ & Dichlorodifluoromethane & 0.50 & $\mathrm{U}$ \\
\hline $74-87-3$ & Chloromethane & 0.50 & $\mathrm{U}$ \\
\hline $75-01-4$ & Vinyl chloride & 0.50 & $\mathrm{U}$ \\
\hline $74-83-9$ & Bromomethane & 0.045 & $\mathrm{~J}$ \\
\hline $75-00-3$ & Chloroethane & 0.50 & $\mathrm{U}$ \\
\hline $75-69-4$ & Trichlorofluoromethane & 0.50 & $\mathrm{U}$ \\
\hline $75-35-4$ & 1,1-Dichloroethene & 0.50 & $\mathrm{U}$ \\
\hline $76-13-1$ & 1,1,2-Trichloro-1,2,2-trifluoroethane & 0.50 & $\mathrm{U}$ \\
\hline $67-64-1$ & Acetone & 5.0 & $\mathrm{U}$ \\
\hline $75-15-0$ & Carbon disulfide & 0.50 & $\mathrm{U}$ \\
\hline $79-20-9$ & Methyl acetate & 0.50 & $\mathrm{U}$ \\
\hline $75-09-2$ & Methylene Chloride & 0.12 & $\mathrm{~J}$ \\
\hline $156-60-5$ & trans-1,2-Dichloroethene & 0.50 & $\mathrm{U}$ \\
\hline $1634-04-4$ & Methyl tert-butyl ether & 0.50 & $\mathrm{U}$ \\
\hline $75-34-3$ & 1,1-Dichloroethane & 0.50 & $\mathrm{U}$ \\
\hline $156-59-2$ & cis-1,2-Dichloroethene & 0.50 & $\mathrm{U}$ \\
\hline $78-93-3$ & 2-Butanone & 5.0 & $\mathrm{U}$ \\
\hline $74-97-5$ & Bromochloromethane & 0.50 & $\mathrm{U}$ \\
\hline $67-66-3$ & Chloroform & 0.50 & $\mathrm{U}$ \\
\hline $71-55-6$ & $1,1,1$-Trichloroethane & 0.50 & $\mathrm{U}$ \\
\hline $110-82-7$ & Cyclohexane & 0.50 & $\mathrm{U}$ \\
\hline $56-23-5$ & Carbon tetrachloride & 0.50 & $\mathrm{U}$ \\
\hline $71-43-2$ & Benzene & 0.50 & $\mathrm{U}$ \\
\hline $107-06-2$ & 1,2-Dichloroethane & 0.50 & $\mathrm{U}$ \\
\hline
\end{tabular}

Report 1,4-Dioxane for Low-Medium VOA analysis only 
Lab Name: TESTAMERICA BURLINGTON

Lab Code: STLV Case No.: EVERES Mod. Ref No.:
Contract: 8E-00302 SDG No.: 16407-1
Matrix: (SOIL/SED/WATER)

Water

Sample wt/vol: 25.0 $(\mathrm{g} / \mathrm{mL}) \quad \mathrm{mL}$

Level: (TRACE/LOW/MED)

TRACE

\% Moisture: not dec.

GC Column: DB-624

Soil Extract Volume: ID $: 0.20 \quad(\mathrm{~mm})$

Purge Volume: 25.0
(uL)

$(\mathrm{mL})$
Lab Sample ID: MB 200-55472/4

Lab File ID: DJPD04.D

Date Received:

Date Analyzed: 05/13/2013

Dilution Factor: 1.0

Soil Aliquot Volume: $(u L)$

\begin{tabular}{|c|c|c|c|}
\hline CAS NO. & COMPOUND & $\begin{array}{l}\text { CONCENTRATION UNITS: } \\
(\mathrm{ug} / \mathrm{L} \text { or } \mathrm{ug} / \mathrm{kg}) \mathrm{ug} / \mathrm{L} \\
\end{array}$ & $Q$ \\
\hline $79-01-6$ & Trichloroethene & 0.50 & $\mathrm{U}$ \\
\hline $108-87-2$ & Methylcyclohexane & 0.50 & $\mathrm{U}$ \\
\hline $78-87-5$ & 1,2-Dichloropropane & 0.50 & $\mathrm{U}$ \\
\hline $75-27-4$ & Bromodichloromethane & 0.50 & $\mathrm{U}$ \\
\hline $10061-01-5$ & cis-1,3-Dichloropropene & 0.50 & $\mathrm{U}$ \\
\hline $108-10-1$ & 4-Methyl-2-pentanone & 5.0 & $\mathrm{U}$ \\
\hline $108-88-3$ & Toluene & 0.50 & $\mathrm{U}$ \\
\hline $10061-02-6$ & trans-1,3-Dichloropropene & 0.50 & $\mathrm{U}$ \\
\hline $79-00-5$ & $1,1,2$-Trichloroethane & 0.50 & $\mathrm{U}$ \\
\hline $127-18-4$ & Tetrachloroethene & 0.50 & $\mathrm{U}$ \\
\hline $591-78-6$ & 2-Hexanone & 5.0 & $\mathrm{U}$ \\
\hline $124-48-1$ & Dibromochloromethane & 0.50 & $\mathrm{U}$ \\
\hline $106-93-4$ & 1,2-Dibromoethane & 0.50 & $\mathrm{U}$ \\
\hline $108-90-7$ & Chlorobenzene & 0.50 & $\mathrm{U}$ \\
\hline $100-41-4$ & Ethylbenzene & 0.50 & $\mathrm{U}$ \\
\hline $95-47-6$ & o-Xylene & 0.50 & $\mathrm{U}$ \\
\hline $179601-23-1$ & $\mathrm{~m}, \mathrm{p}$-Xylene & 0.50 & $\mathrm{U}$ \\
\hline $100-42-5$ & Styrene & 0.50 & $\mathrm{U}$ \\
\hline $75-25-2$ & Bromoform & 0.50 & $\mathrm{U}$ \\
\hline $98-82-8$ & Isopropylbenzene & 0.50 & $\mathrm{U}$ \\
\hline $79-34-5$ & $1,1,2,2$-Tetrachloroethane & 0.50 & $\mathrm{U}$ \\
\hline $541-73-1$ & 1,3-Dichlorobenzene & 0.50 & $\mathrm{U}$ \\
\hline $106-46-7$ & 1,4-Dichlorobenzene & 0.50 & $\mathrm{U}$ \\
\hline $95-50-1$ & 1,2-Dichlorobenzene & 0.50 & $\mathrm{U}$ \\
\hline $96-12-8$ & 1,2-Dibromo-3-Chloropropane & 0.50 & $\mathrm{U}$ \\
\hline $120-82-1$ & $1,2,4$-Trichlorobenzene & 0.50 & $\mathrm{U}$ \\
\hline $87-61-6$ & 1,2,3-Trichlorobenzene & 0.50 & $\mathrm{U}$ \\
\hline
\end{tabular}




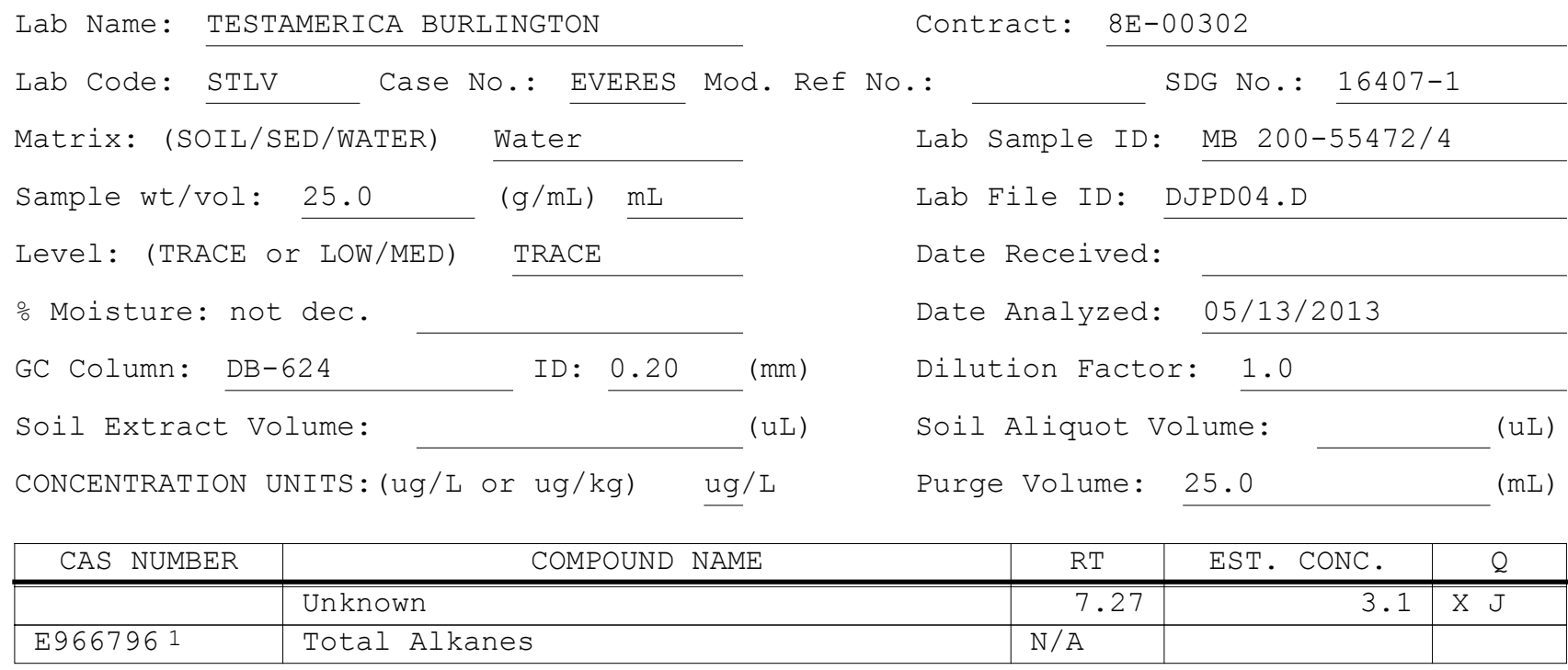


Lab Name: TESTAMERICA BURLINGTON

Contract: $\quad 8 \mathrm{E}-00302$

Lab Code: STLV Case No.: EVERES Mod. Ref No.: SDG No.: 16407-1

Matrix: (SOIL/SED/WATER)

Water

Sample wt/vol: 25.0 $(\mathrm{g} / \mathrm{mL}) \quad \mathrm{mL}$

Level: (TRACE/LOW/MED) TRACE

\% Moisture: not dec.

GC Column: DB-624

Soil Extract Volume: ID $: 0.20 \quad(\mathrm{~mm})$

Purge Volume: 25.0
Lab Sample ID: 200-16407-6

Lab File ID: DJPD12.D

Date Received:

Date Analyzed: 05/13/2013

Dilution Factor: 1.0

Soil Aliquot Volume: (uL)

\begin{tabular}{|c|c|c|c|}
\hline CAS NO. & COMPOUND & $\begin{array}{l}\text { CONCENTRATION UNITS: } \\
(\mathrm{ug} / \mathrm{L} \text { or } \mathrm{ug} / \mathrm{kg}) \mathrm{ug} / \mathrm{L}\end{array}$ & Q \\
\hline $75-71-8$ & Dichlorodifluoromethane & 0.50 & $\overline{\mathrm{U}}$ \\
\hline $74-87-3$ & Chloromethane & 0.50 & $\mathrm{U}$ \\
\hline $75-01-4$ & Vinyl chloride & 0.50 & $\mathrm{U}$ \\
\hline $74-83-9$ & Bromomethane & 0.50 & $\mathrm{U}$ \\
\hline $75-00-3$ & Chloroethane & 0.50 & $\mathrm{U}$ \\
\hline $75-69-4$ & Trichlorofluoromethane & 0.50 & $\mathrm{U}$ \\
\hline $75-35-4$ & 1,1-Dichloroethene & 0.50 & $\mathrm{U}$ \\
\hline $76-13-1$ & $1,1,2$-Trichloro-1,2,2-trifluoroethane & 0.50 & $\mathrm{U}$ \\
\hline $67-64-1$ & Acetone & 5.0 & $\mathrm{U}$ \\
\hline $75-15-0$ & Carbon disulfide & 0.50 & $\mathrm{U}$ \\
\hline $79-20-9$ & Methyl acetate & 0.50 & $\mathrm{U}$ \\
\hline $75-09-2$ & Methylene Chloride & 0.50 & $\mathrm{U}$ \\
\hline $156-60-5$ & trans-1,2-Dichloroethene & 0.50 & $\mathrm{U}$ \\
\hline $1634-04-4$ & Methyl tert-butyl ether & 0.50 & $\mathrm{U}$ \\
\hline $75-34-3$ & 1,1-Dichloroethane & 0.50 & $\mathrm{U}$ \\
\hline $156-59-2$ & cis-1,2-Dichloroethene & 0.50 & $\mathrm{U}$ \\
\hline $78-93-3$ & 2-Butanone & 5.0 & $\mathrm{U}$ \\
\hline $74-97-5$ & Bromochloromethane & 0.50 & $\mathrm{U}$ \\
\hline $67-66-3$ & Chloroform & 0.50 & $\mathrm{U}$ \\
\hline $71-55-6$ & 1,1,1-Trichloroethane & 0.50 & $\mathrm{U}$ \\
\hline $110-82-7$ & Cyclohexane & 0.50 & $\mathrm{U}$ \\
\hline $56-23-5$ & Carbon tetrachloride & 0.50 & $\mathrm{U}$ \\
\hline $71-43-2$ & Benzene & 0.50 & $\mathrm{U}$ \\
\hline $107-06-2$ & 1,2-Dichloroethane & 0.50 & $\mathrm{U}$ \\
\hline
\end{tabular}

Report 1,4-Dioxane for Low-Medium VOA analysis only 
Lab Name: TESTAMERICA BURLINGTON

Lab Code: STLV Case No.: EVERES Mod. Ref No.:
Contract: $8 \mathrm{E}-00302$ SDG No.: 16407-1
Matrix: (SOIL/SED/WATER)

Water

Sample wt/vol: 25.0 $(\mathrm{g} / \mathrm{mL}) \quad \mathrm{mL}$

Level : (TRACE/LOW/MED)

TRACE

\% Moisture: not dec.

GC Column: DB-624

Soil Extract Volume: ID $: 0.20 \quad(\mathrm{~mm})$

Purge Volume: 25.0
(uL)

$(\mathrm{mL})$
Lab Sample ID: 200-16407-6

Lab File ID: DJPD12.D

Date Received:

Date Analyzed: 05/13/2013

Dilution Factor: 1.0

Soil Aliquot Volume:

\begin{tabular}{|c|c|c|c|}
\hline CAS NO. & COMPOUND & $\begin{array}{l}\text { CONCENTRATION UNITS: } \\
(\mathrm{ug} / \mathrm{L} \text { or } \mathrm{ug} / \mathrm{kg}) \mathrm{ug} / \mathrm{L}\end{array}$ & \& \\
\hline $79-01-6$ & Trichloroethene & 0.50 & $\overline{\mathrm{U}}$ \\
\hline $108-87-2$ & Methylcyclohexane & 0.50 & $\mathrm{U}$ \\
\hline $78-87-5$ & 1,2-Dichloropropane & 0.50 & $\mathrm{U}$ \\
\hline $75-27-4$ & Bromodichloromethane & 0.50 & $\mathrm{U}$ \\
\hline $10061-01-5$ & cis-1,3-Dichloropropene & 0.50 & $\mathrm{U}$ \\
\hline $108-10-1$ & 4-Methyl-2-pentanone & 5.0 & $\mathrm{U}$ \\
\hline $108-88-3$ & Toluene & 0.50 & $\mathrm{U}$ \\
\hline $10061-02-6$ & trans-1,3-Dichloropropene & 0.50 & $\mathrm{U}$ \\
\hline $79-00-5$ & $1,1,2$-Trichloroethane & 0.50 & $\mathrm{U}$ \\
\hline $127-18-4$ & Tetrachloroethene & 0.50 & $\mathrm{U}$ \\
\hline $591-78-6$ & 2-Hexanone & 5.0 & $\mathrm{U}$ \\
\hline $124-48-1$ & Dibromochloromethane & 0.50 & $\mathrm{U}$ \\
\hline $106-93-4$ & 1,2-Dibromoethane & 0.50 & $\mathrm{U}$ \\
\hline $108-90-7$ & Chlorobenzene & 0.50 & $\mathrm{U}$ \\
\hline $100-41-4$ & Ethylbenzene & 0.50 & $\mathrm{U}$ \\
\hline $95-47-6$ & o-Xylene & 0.50 & $\mathrm{U}$ \\
\hline $179601-23-1$ & m,p-Xylene & 0.50 & $\mathrm{U}$ \\
\hline $100-42-5$ & Styrene & 0.50 & $\mathrm{U}$ \\
\hline $75-25-2$ & Bromoform & 0.50 & $\mathrm{U}$ \\
\hline $98-82-8$ & Isopropylbenzene & 0.50 & $\mathrm{U}$ \\
\hline $79-34-5$ & $1,1,2,2$-Tetrachloroethane & 0.50 & $\mathrm{U}$ \\
\hline $541-73-1$ & 1,3-Dichlorobenzene & 0.50 & $\mathrm{U}$ \\
\hline $106-46-7$ & 1,4-Dichlorobenzene & 0.50 & $\mathrm{U}$ \\
\hline $95-50-1$ & 1,2-Dichlorobenzene & 0.50 & $\mathrm{U}$ \\
\hline $96-12-8$ & 1,2-Dibromo-3-Chloropropane & 0.50 & $\mathrm{U}$ \\
\hline $120-82-1$ & 1,2,4-Trichlorobenzene & 0.50 & $\mathrm{U}$ \\
\hline $87-61-6$ & $1,2,3$-Trichlorobenzene & 0.50 & $\mathrm{U}$ \\
\hline
\end{tabular}




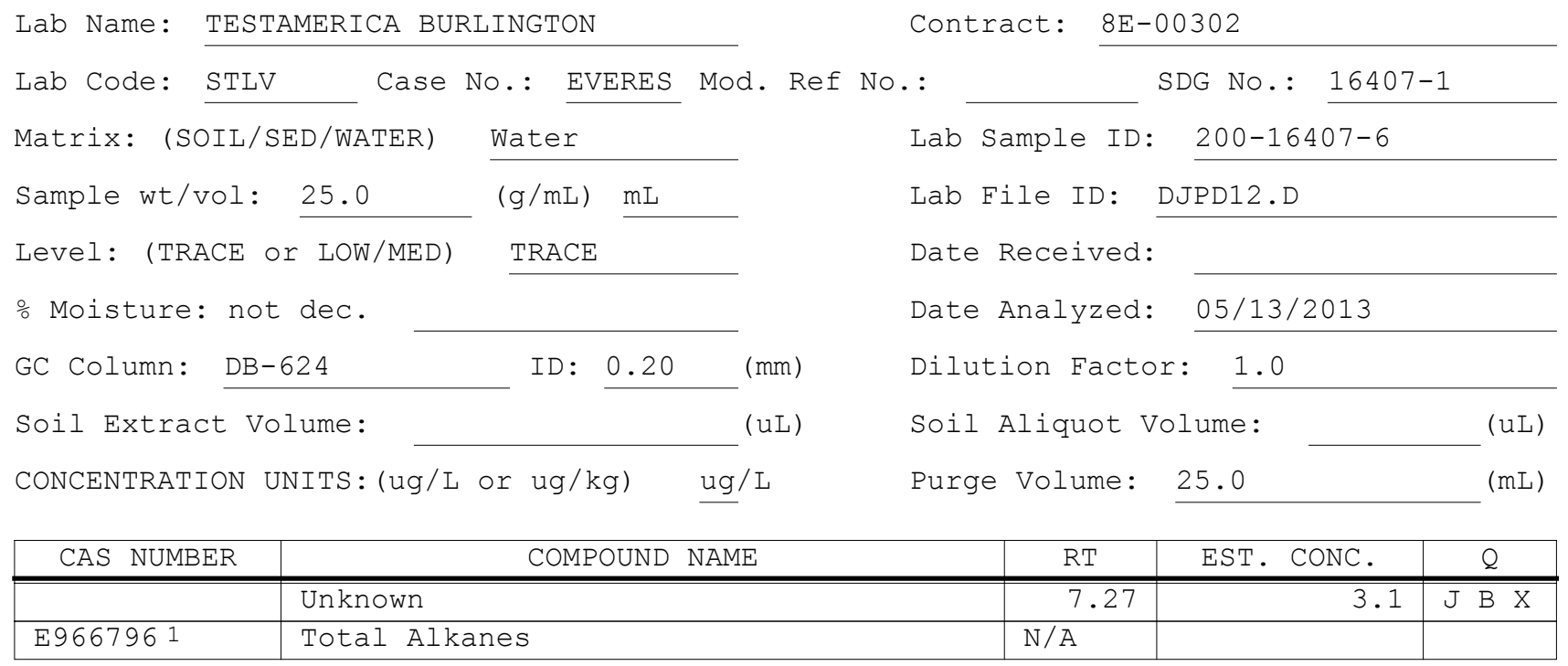


Lab Name: TESTAMERICA BURLINGTON Case No.: EVERES Mod. Ref No.:
Lab Code: STLV

Contract: $8 \mathrm{E}-00302$

\section{Matrix: (SOIL/SED/WATER)}

Water

Sample wt/vol: 25.0 $(\mathrm{g} / \mathrm{mL}) \quad \mathrm{mL}$

Level: (TRACE/LOW/MED)

TRACE

\% Moisture: not dec.

GC Column: DB-624 ID $: 0.20 \quad(\mathrm{~mm})$

Soil Extract Volume: (uL) $(\mathrm{mL})$

Lab File ID: DJPD08.D

Date Received:

Dilution Factor: 1.0

Soil Aliquot Volume: (mI) SDG No.: 16407-1

Lab Sample ID: VIBLK 200-55472/8

Date Analyzed: 05/13/2013

\begin{tabular}{|c|c|c|c|}
\hline CAS NO. & COMPOUND & $\begin{array}{l}\text { CONCENTRATION UNITS: } \\
(\mathrm{ug} / \mathrm{L} \text { or } \mathrm{ug} / \mathrm{kg}) \mathrm{ug} / \mathrm{L}\end{array}$ & Q \\
\hline $75-71-8$ & Dichlorodifluoromethane & 0.50 & $\overline{\mathrm{U}}$ \\
\hline $74-87-3$ & Chloromethane & 0.50 & $\mathrm{U}$ \\
\hline $75-01-4$ & Vinyl chloride & 0.50 & $\mathrm{U}$ \\
\hline $74-83-9$ & Bromomethane & 0.50 & $\mathrm{U}$ \\
\hline $75-00-3$ & Chloroethane & 0.50 & $\mathrm{U}$ \\
\hline $75-69-4$ & Trichlorofluoromethane & 0.50 & $\mathrm{U}$ \\
\hline $75-35-4$ & 1,1-Dichloroethene & 0.50 & $\mathrm{U}$ \\
\hline $76-13-1$ & $1,1,2$-Trichloro-1,2,2-trifluoroethane & 0.50 & $\mathrm{U}$ \\
\hline $67-64-1$ & Acetone & 5.0 & $\mathrm{U}$ \\
\hline $75-15-0$ & Carbon disulfide & 0.50 & $\mathrm{U}$ \\
\hline $79-20-9$ & Methyl acetate & 0.50 & $\mathrm{U}$ \\
\hline $75-09-2$ & Methylene Chloride & 0.17 & $\mathrm{~J} B$ \\
\hline $156-60-5$ & trans-1,2-Dichloroethene & 0.50 & $\mathrm{U}$ \\
\hline $1634-04-4$ & Methyl tert-butyl ether & 0.50 & $\mathrm{U}$ \\
\hline $75-34-3$ & 1,1-Dichloroethane & 0.50 & $\mathrm{U}$ \\
\hline $156-59-2$ & cis-1,2-Dichloroethene & 0.50 & $\mathrm{U}$ \\
\hline $78-93-3$ & 2-Butanone & 5.0 & $\mathrm{U}$ \\
\hline $74-97-5$ & Bromochloromethane & 0.50 & $\mathrm{U}$ \\
\hline $67-66-3$ & Chloroform & 0.50 & $\mathrm{U}$ \\
\hline $71-55-6$ & $1,1,1$-Trichloroethane & 0.50 & $\mathrm{U}$ \\
\hline $110-82-7$ & Cyclohexane & 0.50 & $\mathrm{U}$ \\
\hline $56-23-5$ & Carbon tetrachloride & 0.028 & $\mathrm{~J}$ \\
\hline $71-43-2$ & Benzene & 0.50 & $\mathrm{U}$ \\
\hline $107-06-2$ & 1,2-Dichloroethane & 0.50 & $\mathrm{U}$ \\
\hline
\end{tabular}


Lab Name: TESTAMERICA BURLINGTON

Lab Code: STLV Case No.: EVERES Mod. Ref No.:
Contract: $8 \mathrm{E}-00302$ SDG No.: 16407-1
Matrix: (SOIL/SED/WATER)

Water

Sample wt/vol: 25.0 $(\mathrm{g} / \mathrm{mL}) \quad \mathrm{mL}$

Level: (TRACE/LOW/MED)

TRACE

\% Moisture: not dec.

GC Column: DB-624

Soil Extract Volume: ID $: 0.20 \quad(\mathrm{~mm})$

Purge Volume: 25.0
(uL)

$(\mathrm{mL})$
Lab Sample ID: VIBLK 200-55472/8

Lab File ID: DJPD08.D

Date Received:

Date Analyzed: 05/13/2013

Dilution Factor: 1.0

Soil Aliquot Volume: $(u L)$

\begin{tabular}{|c|c|c|c|}
\hline CAS NO. & COMPOUND & $\begin{array}{l}\text { CONCENTRATION UNITS: } \\
(\mathrm{ug} / \mathrm{L} \text { or } \mathrm{ug} / \mathrm{kg}) \mathrm{ug} / \mathrm{L} \\
\end{array}$ & $Q$ \\
\hline $79-01-6$ & Trichloroethene & 0.50 & $\mathrm{U}$ \\
\hline $108-87-2$ & Methylcyclohexane & 0.50 & $\mathrm{U}$ \\
\hline $78-87-5$ & 1,2-Dichloropropane & 0.50 & $\mathrm{U}$ \\
\hline $75-27-4$ & Bromodichloromethane & 0.50 & $\mathrm{U}$ \\
\hline $10061-01-5$ & cis-1,3-Dichloropropene & 0.50 & $\mathrm{U}$ \\
\hline $108-10-1$ & 4-Methyl-2-pentanone & 5.0 & $\mathrm{U}$ \\
\hline $108-88-3$ & Toluene & 0.50 & $\mathrm{U}$ \\
\hline $10061-02-6$ & trans-1,3-Dichloropropene & 0.50 & $\mathrm{U}$ \\
\hline $79-00-5$ & $1,1,2$-Trichloroethane & 0.50 & $\mathrm{U}$ \\
\hline $127-18-4$ & Tetrachloroethene & 0.50 & $\mathrm{U}$ \\
\hline $591-78-6$ & 2-Hexanone & 5.0 & $\mathrm{U}$ \\
\hline $124-48-1$ & Dibromochloromethane & 0.50 & $\mathrm{U}$ \\
\hline $106-93-4$ & 1,2-Dibromoethane & 0.50 & $\mathrm{U}$ \\
\hline $108-90-7$ & Chlorobenzene & 0.50 & $\mathrm{U}$ \\
\hline $100-41-4$ & Ethylbenzene & 0.50 & $\mathrm{U}$ \\
\hline $95-47-6$ & o-Xylene & 0.50 & $\mathrm{U}$ \\
\hline $179601-23-1$ & $\mathrm{~m}, \mathrm{p}$-Xylene & 0.50 & $\mathrm{U}$ \\
\hline $100-42-5$ & Styrene & 0.50 & $\mathrm{U}$ \\
\hline $75-25-2$ & Bromoform & 0.50 & $\mathrm{U}$ \\
\hline $98-82-8$ & Isopropylbenzene & 0.50 & $\mathrm{U}$ \\
\hline $79-34-5$ & $1,1,2,2$-Tetrachloroethane & 0.50 & $\mathrm{U}$ \\
\hline $541-73-1$ & 1,3-Dichlorobenzene & 0.50 & $\mathrm{U}$ \\
\hline $106-46-7$ & 1,4-Dichlorobenzene & 0.50 & $\mathrm{U}$ \\
\hline $95-50-1$ & 1,2-Dichlorobenzene & 0.50 & $\mathrm{U}$ \\
\hline $96-12-8$ & 1,2-Dibromo-3-Chloropropane & 0.50 & $\mathrm{U}$ \\
\hline $120-82-1$ & $1,2,4$-Trichlorobenzene & 0.50 & $\mathrm{U}$ \\
\hline $87-61-6$ & 1,2,3-Trichlorobenzene & 0.50 & $\mathrm{U}$ \\
\hline
\end{tabular}




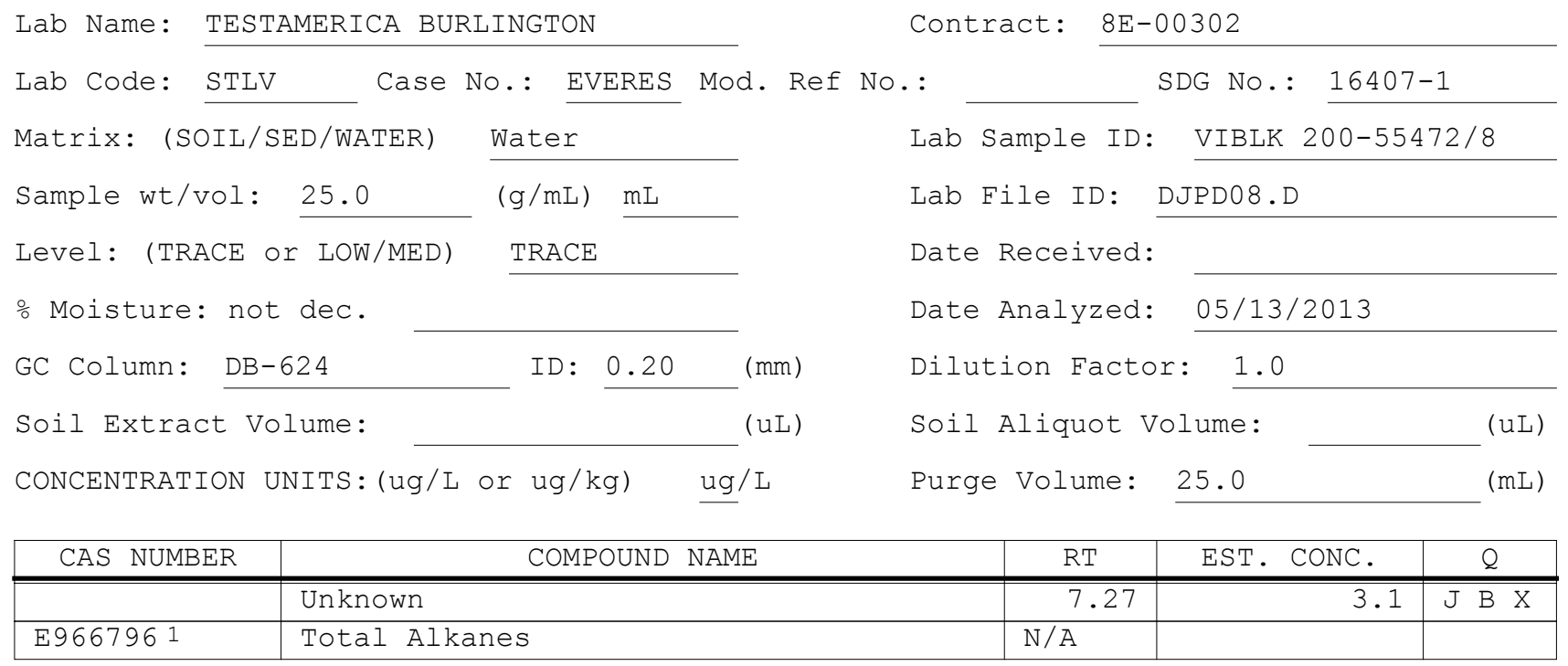


Lab Name: TestAmerica Burlington

SDG No.: 16407-1

Instrument ID: D.i

Lab Sample ID: IC 200-55044/3

Date Analyzed: 05/01/13 11:32
Job No.: 200-16407-1

\section{Analysis Batch Number: 55044}

Client Sample ID:

Lab File ID: djp03.d
ID $: 0.2(\mathrm{~mm})$

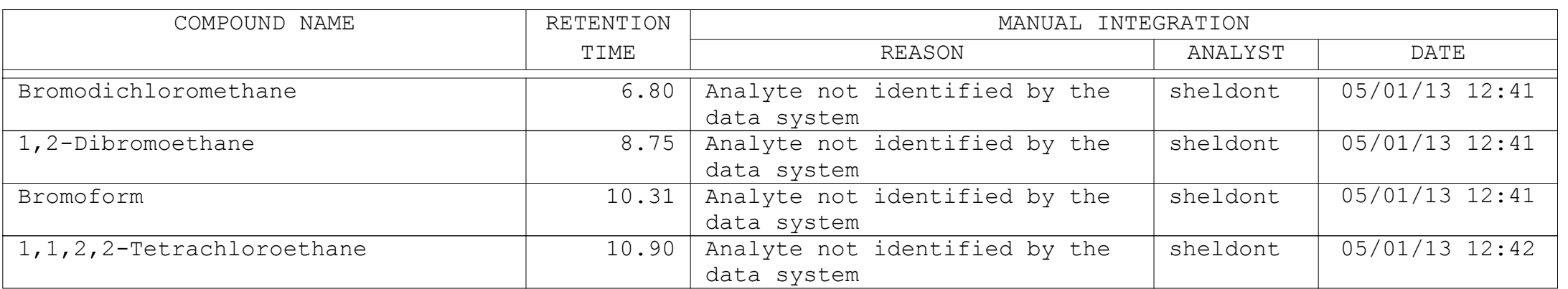


Lab Name: TestAmerica Burlington

Job No.: 200-16407-1

SDG No.: 16407-1

Instrument ID: D.i

Analysis Batch Number: 55472

Lab Sample ID: 200-16407-1

Client Sample ID: EVMW1-W-29823

Date Analyzed: 05/13/13 11:44

Lab File ID: djpd05.d

GC Column: DB-624

ID $: 0.2(\mathrm{~mm})$

\begin{tabular}{|c|c|c|c|c|}
\hline \multirow[t]{2}{*}{ COMPOUND NAME } & \multirow{2}{*}{$\begin{array}{l}\text { RETENTION } \\
\text { TIME }\end{array}$} & \multicolumn{3}{|c|}{ MANUAL INTEGRATION } \\
\hline & & REASON & ANALYST & DATE \\
\hline Ethylbenzene & 9.48 & $\begin{array}{l}\text { Analyte misidentified by the } \\
\text { data system }\end{array}$ & sheldont & $05 / 13 / 13 \quad 12: 13$ \\
\hline o-Xylene & 10.10 & $\begin{array}{l}\text { Analyte misidentified by the } \\
\text { data system }\end{array}$ & sheldont & $05 / 13 / 13 \quad 12: 13$ \\
\hline
\end{tabular}

Lab Sample ID: 200-16407-2

Client Sample ID: EVMW4-W-29826

Date Analyzed: 05/13/13 12:56

Lab File ID: djpd07.d

GC Column: DB-624

ID $: 0.2(\mathrm{~mm})$

\begin{tabular}{|c|c|c|c|c|}
\hline \multirow[t]{2}{*}{ COMPOUND NAME } & RETENTION & \multicolumn{3}{|c|}{ MANUAL INTEGRATION } \\
\hline & TIME & REASON & ANALYST & DATE \\
\hline o-Xylene & 10.10 & $\begin{array}{l}\text { Analyte misidentified by the } \\
\text { data system }\end{array}$ & sheldont & $05 / 13 / 13 \quad 13: 13$ \\
\hline
\end{tabular}

Lab Sample ID: 200-16407-3

Client Sample ID: EVSB63-W-29832

Date Analyzed: 05/13/13 13:43

Lab File ID: djpd09.d

GC Column: DB-624

ID : $0.2(\mathrm{~mm})$

\begin{tabular}{|l|c|l|c|c|}
\hline \multicolumn{1}{|c|}{ COMPOUND NAME } & RETENTION & \multicolumn{2}{|c|}{ MANUAL INTEGRATION } \\
\cline { 3 - 4 } & TIME & \multicolumn{1}{|c|}{ REASON } & \multicolumn{1}{|c|}{ DATE } \\
\hline Ethylbenzene & 9.48 & $\begin{array}{l}\text { Analyte misidentified by the } \\
\text { data system }\end{array}$ & sheldont & $05 / 13 / 13$ 14:03 \\
\hline o-Xylene & 10.10 & $\begin{array}{l}\text { Analyte misidentified by the } \\
\text { data system }\end{array}$ & sheldont & $05 / 13 / 13$ 14:07 \\
\hline
\end{tabular}

Lab Sample ID: 200-16407-5

Client Sample ID: EVSB80-W-29835

Date Analyzed: 05/13/13 14:31

Lab File ID: djpdi1.d

GC Column: DB-624

ID $: 0.2(\mathrm{~mm})$

\begin{tabular}{|c|c|c|c|c|}
\hline \multirow{2}{*}{ COMPOUND NAME } & RETENTION & \multicolumn{2}{|c|}{ MANUAL INTEGRATION } \\
\cline { 3 - 5 } & TIME & REASON & ANALYST & \multicolumn{2}{|c|}{ DATE } \\
\hline o-Xylene & 10.10 & $\begin{array}{l}\text { Analyte misidentified by the } \\
\text { data system }\end{array}$ & sheldont & $05 / 13 / 13$ 14:47 \\
\hline
\end{tabular}


Lab Name: TestAmerica Burlington

Job No.: 200-16407-1

SDG No.: 16407-1

\begin{tabular}{|c|c|c|c|c|}
\hline $\begin{array}{c}\text { Reagent } \\
\text { Container }\end{array}$ & $\begin{array}{l}\text { Reagent } \\
\text { ID }\end{array}$ & $\begin{array}{c}\text { Reagent } \\
\text { Description }\end{array}$ & $\begin{array}{c}\text { Preparation } \\
\text { Date }\end{array}$ & $\begin{array}{c}\text { Expiration } \\
\text { Date }\end{array}$ \\
\hline 434952 & VMBEBw_00010 & BFB TUNE 25 PPM & $12 / 06 / 2012$ & $06 / 06 / 2013$ \\
\hline 485739 & VMSOMTRISw_00070 & SOM TR ISTD 20 PPM & $04 / 22 / 2013$ & $05 / 21 / 2013$ \\
\hline 489667 & VMSOMTRCALW_00059 & SOM TR CAL 20 PPM & $05 / 01 / 2013$ & $06 / 01 / 2013$ \\
\hline 489692 & VMSOMTRSUW_00063 & SOM TR DMC 20 PPM & $05 / 01 / 2013$ & $06 / 01 / 2013$ \\
\hline
\end{tabular}


SDG No.: 16407-1

\begin{tabular}{|c|c|c|c|c|c|c|c|c|}
\hline \multirow[b]{2}{*}{ Reagent ID } & \multirow[b]{2}{*}{$\begin{array}{l}\text { Exp } \\
\text { Date }\end{array}$} & \multirow[b]{2}{*}{$\begin{array}{l}\text { Prep } \\
\text { Date }\end{array}$} & \multirow[b]{2}{*}{$\begin{array}{c}\text { Dilutant } \\
\text { Used }\end{array}$} & \multirow{2}{*}{$\begin{array}{c}\text { Reagent } \\
\text { Final } \\
\text { Volume }\end{array}$} & \multicolumn{2}{|c|}{ Parent Reagent } & \multirow[b]{2}{*}{ Analyte } & \multirow[b]{2}{*}{ Concentration } \\
\hline & & & & & Reagent ID & $\begin{array}{l}\text { Volume } \\
\text { Added }\end{array}$ & & \\
\hline VMBFBW 00010 & $06 / 06 / 13$ & $12 / 06 / 12$ & METHANOL, LOt 114965 & $25 \mathrm{~mL}$ & VMBFBS 00010 & $125 \mathrm{uL}$ & $\mathrm{BFB}$ & $25 \mathrm{ug} / \mathrm{mL}$ \\
\hline .VMBEBS_ 00010 & $06 / 07 / 16$ & & RESTEK, Lot A082468 & & (Purchased & & $\mathrm{BFB}$ & $5000 \mathrm{ug} / \mathrm{mL}$ \\
\hline \multirow{44}{*}{ VMSOMTRCALw_00059 } & \multirow[t]{44}{*}{$06 / 01 / 13$} & \multirow[t]{44}{*}{$05 / 01 / 13$} & \multirow[t]{44}{*}{ METHANOL, Lot 124454} & \multirow[t]{44}{*}{$4000 \mathrm{uL}$} & \multirow{6}{*}{ VM8260CALbs_00122 } & \multirow{6}{*}{$40 \mathrm{uL}$} & Bromomethane & $20 \mathrm{ug} / \mathrm{mL}$ \\
\hline & & & & & & & Chloroethane & $20 \mathrm{ug} / \mathrm{mL}$ \\
\hline & & & & & & & Chloromethane & $20 \mathrm{ug} / \mathrm{mL}$ \\
\hline & & & & & & & Dichlorodifluoromethane & $20 \mathrm{ug} / \mathrm{mL}$ \\
\hline & & & & & & & Trichlorofluoromethane & $20 \mathrm{ug} / \mathrm{mL}$ \\
\hline & & & & & & & Vinyl chloride & $20 \mathrm{ug} / \mathrm{mL}$ \\
\hline & & & & & \multirow[t]{38}{*}{ VMSOMCALas_00012 } & \multirow[t]{38}{*}{$40 \mathrm{uL}$} & 1,1,1-Trichloroethane & $20 \mathrm{ug} / \mathrm{mL}$ \\
\hline & & & & & & & $1,1,2,2$-Tetrachloroethane & $20 \mathrm{ug} / \mathrm{mL}$ \\
\hline & & & & & & & $\begin{array}{l}\text { 1,1,2-Trichloro-1,2,2-trifluor } \\
\text { oethane }\end{array}$ & $20 \mathrm{ug} / \mathrm{mL}$ \\
\hline & & & & & & & 1,1,2-Trichloroethane & $20 \mathrm{ug} / \mathrm{mL}$ \\
\hline & & & & & & & 1,1-Dichloroethane & $20 \mathrm{ug} / \mathrm{mL}$ \\
\hline & & & & & & & 1,1-Dichloroethene & $20 \mathrm{ug} / \mathrm{mL}$ \\
\hline & & & & & & & 1,2,3-Trichlorobenzene & $20 \mathrm{ug} / \mathrm{mL}$ \\
\hline & & & & & & & 1,2,4-Trichlorobenzene & $20 \mathrm{ug} / \mathrm{mL}$ \\
\hline & & & & & & & 1,2-Dibromo-3-Chloropropane & $20 \mathrm{ug} / \mathrm{mL}$ \\
\hline & & & & & & & 1,2-Dibromoethane & $20 \mathrm{ug} / \mathrm{mL}$ \\
\hline & & & & & & & 1,2-Dichlorobenzene & $20 \mathrm{ug} / \mathrm{mL}$ \\
\hline & & & & & & & 1,2-Dichloroethane & $20 \mathrm{ug} / \mathrm{mL}$ \\
\hline & & & & & & & 1,2-Dichloropropane & $20 \mathrm{ug} / \mathrm{mL}$ \\
\hline & & & & & & & 1,3-Dichlorobenzene & $20 \mathrm{ug} / \mathrm{mL}$ \\
\hline & & & & & & & 1,4-Dichlorobenzene & $20 \mathrm{ug} / \mathrm{mL}$ \\
\hline & & & & & & & Benzene & $20 \mathrm{ug} / \mathrm{mL}$ \\
\hline & & & & & & & Bromochloromethane & $20 \mathrm{ug} / \mathrm{mL}$ \\
\hline & & & & & & & Bromodichloromethane & $20 \mathrm{ug} / \mathrm{mL}$ \\
\hline & & & & & & & Bromoform & $20 \mathrm{ug} / \mathrm{mL}$ \\
\hline & & & & & & & Carbon disulfide & $20 \mathrm{ug} / \mathrm{mL}$ \\
\hline & & & & & & & Carbon tetrachloride & $20 \mathrm{ug} / \mathrm{mL}$ \\
\hline & & & & & & & Chlorobenzene & $20 \mathrm{ug} / \mathrm{mL}$ \\
\hline & & & & & & & Chloroform & $20 \mathrm{ug} / \mathrm{mL}$ \\
\hline & & & & & & & cis-1,2-Dichloroethene & $20 \mathrm{ug} / \mathrm{mL}$ \\
\hline & & & & & & & cis-1,3-Dichloropropene & $20 \mathrm{ug} / \mathrm{mL}$ \\
\hline & & & & & & & Cyclohexane & $20 \mathrm{ug} / \mathrm{mL}$ \\
\hline & & & & & & & Dibromochloromethane & $20 \mathrm{ug} / \mathrm{mL}$ \\
\hline & & & & & & & Ethylbenzene & $20 \mathrm{ug} / \mathrm{mL}$ \\
\hline & & & & & & & Isopropylbenzene & $20 \mathrm{ug} / \mathrm{mL}$ \\
\hline & & & & & & & $\mathrm{m}, \mathrm{p}$-Xylene & $20 \mathrm{ug} / \mathrm{mL}$ \\
\hline & & & & & & & Methyl acetate & $20 \mathrm{ug} / \mathrm{mL}$ \\
\hline & & & & & & & Methyl tert-butyl ether & $20 \mathrm{ug} / \mathrm{mL}$ \\
\hline & & & & & & & Methylcyclohexane & $20 \mathrm{ug} / \mathrm{mL}$ \\
\hline & & & & & & & Methylene Chloride & $20 \mathrm{ug} / \mathrm{mL}$ \\
\hline & & & & & & & o-Xylene & $20 \mathrm{ug} / \mathrm{mL}$ \\
\hline & & & & & & & Styrene & $20 \mathrm{ug} / \mathrm{mL}$ \\
\hline & & & & & & & Tetrachloroethene & $20 \mathrm{ug} / \mathrm{mL}$ \\
\hline & & & & & & & Toluene & $20 \mathrm{ug} / \mathrm{mL}$ \\
\hline
\end{tabular}


SDG No.: 16407-1

\begin{tabular}{|c|c|c|c|c|c|c|c|c|}
\hline \multirow[b]{2}{*}{ Reagent ID } & \multirow[b]{2}{*}{$\begin{array}{l}\text { Exp } \\
\text { Date }\end{array}$} & \multirow[b]{2}{*}{$\begin{array}{l}\text { Prep } \\
\text { Date }\end{array}$} & \multirow[b]{2}{*}{$\begin{array}{l}\text { Dilutant } \\
\text { Used }\end{array}$} & \multirow{2}{*}{$\begin{array}{l}\text { Reagent } \\
\text { Final } \\
\text { Volume }\end{array}$} & \multicolumn{2}{|l|}{ Parent Reagent } & \multirow[b]{2}{*}{ Analyte } & \multirow[b]{2}{*}{ Concentration } \\
\hline & & & & & Reagent ID & $\begin{array}{l}\text { Volume } \\
\text { Added }\end{array}$ & & \\
\hline & & & & & & & trans-1,2-Dichloroethene & $20 \mathrm{ug} / \mathrm{mL}$ \\
\hline & & & & & & & trans-1,3-Dichloropropene & $20 \mathrm{ug} / \mathrm{mL}$ \\
\hline & & & & & & & Trichloroethene & $20 \mathrm{ug} / \mathrm{mL}$ \\
\hline & & & & & \multirow[t]{4}{*}{ VMSOMCALbs_00019 } & \multirow[t]{4}{*}{$160 \mathrm{uL}$} & 2-Butanone & $200 \mathrm{ug} / \mathrm{mL}$ \\
\hline & & & & & & & 2-Hexanone & $200 \mathrm{ug} / \mathrm{mL}$ \\
\hline & & & & & & & 4-Methyl-2-pentanone & $200 \mathrm{ug} / \mathrm{mL}$ \\
\hline & & & & & & & Acetone & $200 \mathrm{ug} / \mathrm{mL}$ \\
\hline & & & & & \multirow[t]{2}{*}{ VMSOMSUas_00058 } & \multirow[t]{2}{*}{$40 \mathrm{uL}$} & Chloroethane-d5 & $20 \mathrm{ug} / \mathrm{mL}$ \\
\hline & & & & & & & Vinyl Chloride-d3 & $20 \mathrm{ug} / \mathrm{mL}$ \\
\hline & & & & & \multirow[t]{2}{*}{ VMSOMSUbs_00085 } & \multirow{2}{*}{$320 \mathrm{uL}$} & 2-Butanone-d5 & $200 \mathrm{ug} / \mathrm{mL}$ \\
\hline & & & & & & & 2-Hexanone-d5 & $200 \mathrm{ug} / \mathrm{mL}$ \\
\hline & & & & & \multirow[t]{9}{*}{ VMSOMSUCS_00035 } & \multirow[t]{9}{*}{40 uL } & $1,1,2,2$-Tetrachloroethane-d2 & $20 \mathrm{ug} / \mathrm{mL}$ \\
\hline & & & & & & & 1,1-Dichloroethene-d2 & $20 \mathrm{ug} / \mathrm{mL}$ \\
\hline & & & & & & & 1,2-Dichlorobenzene-d4 & $20 \mathrm{ug} / \mathrm{mL}$ \\
\hline & & & & & & & 1,2-Dichloroethane-d4 & $20 \mathrm{ug} / \mathrm{mL}$ \\
\hline & & & & & & & 1,2-Dichloropropane-d6 & $20 \mathrm{ug} / \mathrm{mL}$ \\
\hline & & & & & & & Benzene-d6 & $20 \mathrm{ug} / \mathrm{mL}$ \\
\hline & & & & & & & Chloroform-d & $20 \mathrm{ug} / \mathrm{mL}$ \\
\hline & & & & & & & Toluene-d8 & $20 \mathrm{ug} / \mathrm{mL}$ \\
\hline & & & & & & & trans-1,3-Dichloropropene-d4 & $20 \mathrm{ug} / \mathrm{mL}$ \\
\hline \multirow[t]{6}{*}{.VM8260CALbs_00122 } & \multirow[t]{6}{*}{$06 / 01 / 13$} & & RESTEK, Lot A090461 & & (Purchased Reagent & & Bromomethane & $2000 \mathrm{ug} / \mathrm{mL}$ \\
\hline & & & & & & & Chloroethane & $2000 \mathrm{ug} / \mathrm{mL}$ \\
\hline & & & & & & & Chloromethane & $2000 \mathrm{ug} / \mathrm{mL}$ \\
\hline & & & & & & & Dichlorodifluoromethane & $2000 \mathrm{ug} / \mathrm{mL}$ \\
\hline & & & & & & & Trichlorofluoromethane & $2000 \mathrm{ug} / \mathrm{mL}$ \\
\hline & & & & & & & Vinyl chloride & $2000 \mathrm{ug} / \mathrm{mL}$ \\
\hline .VMSOMCALas_00012 & $06 / 30 / 13$ & & Restek, Lot A075266 & & (Purchased Reagent & & 1,1,1-Trichloroethane & $2000 \mathrm{ug} / \mathrm{mL}$ \\
\hline & & & & & & & 1,1,2,2-Tetrachloroethane & $2000 \mathrm{ug} / \mathrm{mL}$ \\
\hline & & & & & & & $\begin{array}{l}\text { 1,1,2-Trichloro-1,2,2-trifluor } \\
\text { oethane }\end{array}$ & $2000 \mathrm{ug} / \mathrm{mL}$ \\
\hline & & & & & & & 1,1,2-Trichloroethane & $2000 \mathrm{ug} / \mathrm{mL}$ \\
\hline & & & & & & & 1,1-Dichloroethane & $2000 \mathrm{ug} / \mathrm{mL}$ \\
\hline & & & & & & & 1,1-Dichloroethene & $2000 \mathrm{ug} / \mathrm{mL}$ \\
\hline & & & & & & & 1,2,3-Trichlorobenzene & $2000 \mathrm{ug} / \mathrm{mL}$ \\
\hline & & & & & & & 1,2,4-Trichlorobenzene & $2000 \mathrm{ug} / \mathrm{mL}$ \\
\hline & & & & & & & 1,2-Dibromo-3-Chloropropane & $2000 \mathrm{ug} / \mathrm{mL}$ \\
\hline & & & & & & & 1,2-Dibromoethane & $2000 \mathrm{ug} / \mathrm{mL}$ \\
\hline & & & & & & & 1,2-Dichlorobenzene & $2000 \mathrm{ug} / \mathrm{mL}$ \\
\hline & & & & & & & 1,2-Dichloroethane & $2000 \mathrm{ug} / \mathrm{mL}$ \\
\hline & & & & & & & 1,2-Dichloropropane & $2000 \mathrm{ug} / \mathrm{mL}$ \\
\hline & & & & & & & 1,3-Dichlorobenzene & $2000 \mathrm{ug} / \mathrm{mL}$ \\
\hline & & & & & & & 1,4-Dichlorobenzene & $2000 \mathrm{ug} / \mathrm{mL}$ \\
\hline & & & & & & & Benzene & $2000 \mathrm{ug} / \mathrm{mL}$ \\
\hline & & & & & & & Bromochloromethane & $2000 \mathrm{ug} / \mathrm{mL}$ \\
\hline & & & & & & & Bromodichloromethane & $2000 \mathrm{ug} / \mathrm{mL}$ \\
\hline & & & & & & & Bromoform & $2000 \mathrm{ug} / \mathrm{mL}$ \\
\hline & & & & & & & Carbon disulfide & $2000 \mathrm{ug} / \mathrm{mL}$ \\
\hline & & & & & & & Carbon tetrachloride & $2000 \mathrm{ug} / \mathrm{mL}$ \\
\hline
\end{tabular}


SDG No.: 16407-1

\begin{tabular}{|c|c|c|c|c|c|c|c|c|}
\hline \multirow[b]{2}{*}{ Reagent ID } & \multirow[b]{2}{*}{$\begin{array}{l}\text { Exp } \\
\text { Date }\end{array}$} & \multirow[b]{2}{*}{$\begin{array}{l}\text { Prep } \\
\text { Date }\end{array}$} & \multirow[b]{2}{*}{$\begin{array}{l}\text { Dilutant } \\
\text { Used }\end{array}$} & \multirow{2}{*}{$\begin{array}{c}\text { Reagent } \\
\text { Final } \\
\text { Volume }\end{array}$} & \multicolumn{2}{|l|}{ Parent Reagent } & \multirow[b]{2}{*}{ Analyte } & \multirow[b]{2}{*}{ Concentration } \\
\hline & & & & & Reagent ID & $\begin{array}{l}\text { Volume } \\
\text { Added }\end{array}$ & & \\
\hline & & & & & & & Chlorobenzene & $2000 \mathrm{ug} / \mathrm{mL}$ \\
\hline & & & & & & & Chloroform & $2000 \mathrm{ug} / \mathrm{mL}$ \\
\hline & & & & & & & cis-1,2-Dichloroethene & $2000 \mathrm{ug} / \mathrm{mL}$ \\
\hline & & & & & & & cis-1,3-Dichloropropene & $2000 \mathrm{ug} / \mathrm{mL}$ \\
\hline & & & & & & & Cyclohexane & $2000 \mathrm{ug} / \mathrm{mL}$ \\
\hline & & & & & & & Dibromochloromethane & $2000 \mathrm{ug} / \mathrm{mL}$ \\
\hline & & & & & & & Ethylbenzene & $2000 \mathrm{ug} / \mathrm{mL}$ \\
\hline & & & & & & & Isopropylbenzene & $2000 \mathrm{ug} / \mathrm{mL}$ \\
\hline & & & & & & & $\mathrm{m}, \mathrm{p}$-Xylene & $2000 \mathrm{ug} / \mathrm{mL}$ \\
\hline & & & & & & & Methyl acetate & $2000 \mathrm{ug} / \mathrm{mL}$ \\
\hline & & & & & & & Methyl tert-butyl ether & $2000 \mathrm{ug} / \mathrm{mL}$ \\
\hline & & & & & & & Methylcyclohexane & $2000 \mathrm{ug} / \mathrm{mL}$ \\
\hline & & & & & & & Methylene Chloride & $2000 \mathrm{ug} / \mathrm{mL}$ \\
\hline & & & & & & & o-Xylene & $2000 \mathrm{ug} / \mathrm{mL}$ \\
\hline & & & & & & & Styrene & $2000 \mathrm{ug} / \mathrm{mL}$ \\
\hline & & & & & & & Tetrachloroethene & $2000 \mathrm{ug} / \mathrm{mL}$ \\
\hline & & & & & & & Toluene & $2000 \mathrm{ug} / \mathrm{mL}$ \\
\hline & & & & & & & trans-1,2-Dichloroethene & $2000 \mathrm{ug} / \mathrm{mL}$ \\
\hline & & & & & & & trans-1,3-Dichloropropene & $2000 \mathrm{ug} / \mathrm{mL}$ \\
\hline & & & & & & & Trichloroethene & $2000 \mathrm{ug} / \mathrm{mL}$ \\
\hline \multirow[t]{4}{*}{.VMSOMCALbS_00019 } & \multirow[t]{4}{*}{$11 / 01 / 13$} & \multirow{4}{*}{\multicolumn{2}{|c|}{ Restek, Lot A084962 }} & & \multirow{2}{*}{\multicolumn{2}{|c|}{ (Purchased Reagent) }} & 2-Butanone & $5000 \mathrm{ug} / \mathrm{mL}$ \\
\hline & & & & & & & 2-Hexanone & $5000 \mathrm{ug} / \mathrm{mL}$ \\
\hline & & & & & & & 4-Methyl-2-pentanone & $5000 \mathrm{ug} / \mathrm{mL}$ \\
\hline & & & & & & & Acetone & $5000 \mathrm{ug} / \mathrm{mL}$ \\
\hline \multirow[t]{2}{*}{.VMSOMSUas_00058 } & \multirow[t]{2}{*}{$11 / 01 / 13$} & \multirow{2}{*}{\multicolumn{2}{|c|}{ Absolute, Lot 030513}} & & \multirow{2}{*}{\multicolumn{2}{|c|}{ (Purchased Reagent) }} & Chloroethane-d5 & $2000 \mathrm{ug} / \mathrm{mL}$ \\
\hline & & & & & & & Vinyl Chloride-d3 & $2000 \mathrm{ug} / \mathrm{mL}$ \\
\hline \multirow[t]{2}{*}{. VMSOMSUbs_00085 } & \multirow[t]{2}{*}{$11 / 01 / 13$} & & Absolute, Lot 101912 & & (Purchased Reage & & 2-Butanone-d5 & $2500 \mathrm{ug} / \mathrm{mL}$ \\
\hline & & & & & & & 2-Hexanone-d5 & $2500 \mathrm{ug} / \mathrm{mL}$ \\
\hline. VMSOMSUCS_00035 & $11 / 01 / 13$ & & Absolute, Lot 040912 & & (Purchased Reage & & $1,1,2,2$-Tetrachloroethane-d2 & $2000 \mathrm{ug} / \mathrm{mL}$ \\
\hline & & & & & & & 1,1-Dichloroethene-d2 & $2000 \mathrm{ug} / \mathrm{mL}$ \\
\hline & & & & & & & 1,2-Dichlorobenzene-d4 & $2000 \mathrm{ug} / \mathrm{mL}$ \\
\hline & & & & & & & 1,2-Dichloroethane-d4 & $2000 \mathrm{ug} / \mathrm{mL}$ \\
\hline & & & & & & & 1,2-Dichloropropane-d6 & $2000 \mathrm{ug} / \mathrm{mL}$ \\
\hline & & & & & & & Benzene-d6 & $2000 \mathrm{ug} / \mathrm{mL}$ \\
\hline & & & & & & & Chloroform-d & $2000 \mathrm{ug} / \mathrm{mL}$ \\
\hline & & & & & & & Toluene-d8 & $2000 \mathrm{ug} / \mathrm{mL}$ \\
\hline & & & & & & & trans-1,3-Dichloropropene-d4 & $2000 \mathrm{ug} / \mathrm{mL}$ \\
\hline VMSOMTRISW 00070 & $05 / 21 / 13$ & $04 / 22 / 13$ & METHANOL, Lot 124454 & $4400 \mathrm{uL}$ & VMSOMISS 00017 & $35.2 \mathrm{uL}$ & 1,4-Dichlorobenzene-d4 & $20 \mathrm{ug} / \mathrm{mL}$ \\
\hline & & & & & & & 1,4-Difluorobenzene & $20 \mathrm{ug} / \mathrm{mL}$ \\
\hline & & & & & & & Chlorobenzene-d5 & $20 \mathrm{ug} / \mathrm{mL}$ \\
\hline .VMSOMISS_00017 & $05 / 21 / 13$ & & RESTEK, Lot A075426 & & (Purchased Reage & & 1,4-Dichlorobenzene-d4 & $2500 \mathrm{ug} / \mathrm{mL}$ \\
\hline & & & & & & & 1,4-Difluorobenzene & $2500 \mathrm{ug} / \mathrm{mL}$ \\
\hline & & & & & & & Chlorobenzene-d5 & $2500 \mathrm{ug} / \mathrm{mL}$ \\
\hline VMSOMTRSUW_00063 & $06 / 01 / 13$ & $05 / 01 / 13$ & METHANOL, Lot 124454 & 4400 uL & VMSOMSUas_00058 & $44 \mathrm{uL}$ & Chloroethane-d5 & $20 \mathrm{ug} / \mathrm{mL}$ \\
\hline & & & & & & & Vinyl Chloride-d3 & $20 \mathrm{ug} / \mathrm{mL}$ \\
\hline & & & & & VMSOMSUbs_00085 & $352 \mathrm{uL}$ & 2-Butanone-d5 & $200 \mathrm{ug} / \mathrm{mL}$ \\
\hline & & & & & & & 2-Hexanone-d5 & $200 \mathrm{ug} / \mathrm{mL}$ \\
\hline
\end{tabular}


SDG No.: 16407-1

\begin{tabular}{|c|c|c|c|c|c|c|c|c|}
\hline \multirow[b]{2}{*}{ Reagent ID } & \multirow[b]{2}{*}{$\begin{array}{l}\text { Exp } \\
\text { Date }\end{array}$} & \multirow[b]{2}{*}{$\begin{array}{l}\text { Prep } \\
\text { Date }\end{array}$} & \multirow[b]{2}{*}{$\begin{array}{c}\text { Dilutant } \\
\text { Used }\end{array}$} & \multirow{2}{*}{$\begin{array}{c}\text { Reagent } \\
\text { Final } \\
\text { Volume }\end{array}$} & \multicolumn{2}{|l|}{ Parent Reagent } & \multirow[b]{2}{*}{ Analyte } & \multirow[b]{2}{*}{ Concentration } \\
\hline & & & & & Reagent ID & $\begin{array}{l}\text { Volume } \\
\text { Added }\end{array}$ & & \\
\hline & & & & & VMSOMSUCS_00035 & $44 \mathrm{uL}$ & $1,1,2,2$-Tetrachloroethane-d2 & $20 \mathrm{ug} / \mathrm{mL}$ \\
\hline & & & & & & & 1,1-Dichloroethene-d2 & $20 \mathrm{ug} / \mathrm{mL}$ \\
\hline & & & & & & & 1,2-Dichlorobenzene-d4 & $20 \mathrm{ug} / \mathrm{mL}$ \\
\hline & & & & & & & 1,2-Dichloroethane-d4 & $20 \mathrm{ug} / \mathrm{mL}$ \\
\hline & & & & & & & 1,2-Dichloropropane-d6 & $20 \mathrm{ug} / \mathrm{mL}$ \\
\hline & & & & & & & Benzene-d6 & $20 \mathrm{ug} / \mathrm{mL}$ \\
\hline & & & & & & & Chloroform-d & $20 \mathrm{ug} / \mathrm{mL}$ \\
\hline & & & & & & & Toluene-d8 & $20 \mathrm{ug} / \mathrm{mL}$ \\
\hline & & & & & & & trans-1,3-Dichloropropene-d4 & $20 \mathrm{ug} / \mathrm{mL}$ \\
\hline \multirow[t]{2}{*}{.VMSOMSUas_00058 } & $11 / 01 / 13$ & \multirow{2}{*}{\multicolumn{3}{|c|}{ Absolute, Lot 030513}} & \multirow{2}{*}{\multicolumn{2}{|c|}{ (Purchased Reagent) }} & Chloroethane-d5 & $2000 \mathrm{ug} / \mathrm{mL}$ \\
\hline & & & & & & & Vinyl Chloride-d3 & $2000 \mathrm{ug} / \mathrm{mL}$ \\
\hline \multirow[t]{2}{*}{.VMSOMSUbs_00085 } & $11 / 01 / 13$ & \multirow{2}{*}{\multicolumn{3}{|c|}{ Absolute, Lot 101912}} & \multirow{2}{*}{\multicolumn{2}{|c|}{ (Purchased Reagent) }} & 2-Butanone-d5 & $2500 \mathrm{ug} / \mathrm{mL}$ \\
\hline & & & & & & & 2-Hexanone-d5 & $2500 \mathrm{ug} / \mathrm{mL}$ \\
\hline \multirow[t]{9}{*}{.VMSOMSUCS_00035 } & $11 / 01 / 13$ & \multirow{9}{*}{\multicolumn{2}{|c|}{ Absolute, Lot 040912}} & & \multirow{9}{*}{\multicolumn{2}{|c|}{ (Purchased Reagent) }} & $1,1,2,2$-Tetrachloroethane-d2 & $2000 \mathrm{ug} / \mathrm{mL}$ \\
\hline & & & & & & & 1,1-Dichloroethene-d2 & $2000 \mathrm{ug} / \mathrm{mL}$ \\
\hline & & & & & & & 1,2-Dichlorobenzene-d4 & $2000 \mathrm{ug} / \mathrm{mL}$ \\
\hline & & & & & & & 1,2-Dichloroethane-d4 & $2000 \mathrm{ug} / \mathrm{mL}$ \\
\hline & & & & & & & 1,2-Dichloropropane-d6 & $2000 \mathrm{ug} / \mathrm{mL}$ \\
\hline & & & & & & & Benzene-d6 & $2000 \mathrm{ug} / \mathrm{mL}$ \\
\hline & & & & & & & Chloroform-d & $2000 \mathrm{ug} / \mathrm{mL}$ \\
\hline & & & & & & & Toluene-d8 & $2000 \mathrm{ug} / \mathrm{mL}$ \\
\hline & & & & & & & trans-1,3-Dichloropropene-d4 & $2000 \mathrm{ug} / \mathrm{mL}$ \\
\hline
\end{tabular}


Argonne

\section{Environmental Science Division}

Argonne National Laboratory

9700 South Cass Avenue, Bldg. 203

Argonne, IL 60439-4843

www.anl.gov 